\title{
HIRZEBRUCH-ZAGIER CYCLES AND TWISTED TRIPLE PRODUCT SELMER GROUPS
}

\author{
YIFENG LIU
}

\begin{abstract}
Let $E$ be an elliptic curve over $\mathbb{Q}$ and $A$ be another elliptic curve over a real quadratic number field. We construct a $\mathbb{Q}$-motive of rank 8 , together with a distinguished class in the associated Bloch-Kato Selmer group, using HirzebruchZagier cycles, that is, graphs of Hirzebruch-Zagier morphisms. We show that, under certain assumptions on $E$ and $A$, the non-vanishing of the central critical value of the (twisted) triple product $L$-function attached to $(E, A)$ implies that the dimension of the associated Bloch-Kato Selmer group of the motive is 0; and the non-vanishing of the distinguished class implies that the dimension of the associated Bloch-Kato Selmer group of the motive is 1 . This can be viewed as the triple product version of Kolyvagin's work on bounding Selmer groups of a single elliptic curve using Heegner points.
\end{abstract}

\section{Contents}

1. Introduction 2

1.1. Main results 2

1.2. Connection with $L$-function and Beilinson-Bloch-Kato conjecture 6

1.3. The case of base change 9

1.4. Analogy with Kolyvagin's work 10

1.5. Strategy of proof $\quad 12$

1.6. Notation and conventions 13

2. Geometry of some modular schemes $\quad 15$

2.1. Algebraic preparation $\quad 15$

2.2. Some preparations on abelian surfaces 18

2.3. Shimura curves 19

2.4. Hilbert modular surfaces 22

2.5. Hirzebruch-Zagier morphisms 26

3. Construction of cohomology classes 30

3.1. Bloch-Kato Selmer group 30

3.2. Twisted triple product Galois representation 31

3.3. Hirzebruch-Zagier classes 33

3.4. Testing factors 37

3.5. Raising levels 39

3.6. Construction of annihilators 42

4. Congruences of Hirzebruch-Zagier classes 44

Date: August 28, 2015.

2010 Mathematics Subject Classification. 11G05, 11R34, 14G35. 
4.1. Local cohomology and Tate duality 44

4.2. Integral Tate cycles 46

4.3. Statement of congruence formulae 47

4.4. Computation of localization, I 48

4.5. Computation of localization, II 52

5. Bounding Selmer groups 56

5.1. Some Galois-theoretical arguments $\quad 56$

5.2. Proof of Theorem 3.17 60

5.3. Application to elliptic curves 64

$\begin{array}{ll}\text { References } & 68\end{array}$

\section{INTRODUCTION}

Let $E$ be an elliptic curve over $\mathbb{Q}$ and $A$ be another elliptic curve over a real quadratic number field. We construct a $\mathbb{Q}$-motive of rank 8, together with a distinguished class in the associated Bloch-Kato Selmer group, using Hirzebruch-Zagier cycles, that is, graphs of Hirzebruch-Zagier morphisms. We show that, under certain assumptions on $E$ and $A$, the non-vanishing of the central critical value of the (twisted) triple product $L$-function attached to $(E, A)$ implies that the dimension of the associated Bloch-Kato Selmer group of $\mathrm{M}_{E, A}$ is 0 ; and the non-vanishing of the distinguished class implies that the dimension of the associated Bloch-Kato Selmer group of $\mathrm{M}_{E, A}$ is 1 . These results are consequences of the Beilinson-Bloch conjecture, the Bloch-Kato conjecture, and the (conjectural) triple product version of the Gross-Zagier formula, connected by the $L$-function as a bridge. Our results can be viewed as the triple product version of the famous work of Kolyvagin [Kol90] on bounding Selmer groups of a single elliptic curve using Heegner points. In the framework of Gan-Gross-Prasad conjecture, the motive in Kolyvagin's case comes from automorphic representations of the pair $\mathrm{SO}(2) \times \mathrm{SO}(3)$, while ours is from the pair $\mathrm{SO}(3) \times \mathrm{SO}(4)$ in which the group $\mathrm{SO}(4)$ is quasi-split but not split.

Our results certainly provide new evidence toward above-mentioned conjectures, which are among central problems in number theory. They provide the first example in the central critical case with both large Hodge-Tate weights (of range $>1$ ) and large rank $(>4)$.

1.1. Main results. Denote by $\mathbb{Q}^{\text {ac }}$ the algebraic closure of $\mathbb{Q}$ in the field $\mathbb{C}$ of complex numbers, $\mathbb{A}$ the ring of adèle of $\mathbb{Q}$, and $\Gamma_{\mathbb{Q}}=\operatorname{Gal}\left(\mathbb{Q}^{\text {ac }} / \mathbb{Q}\right)$ the absolute Galois group of $\mathbb{Q}$. We fix throughout the whole article a real quadratic field $F \subset \mathbb{Q}^{\text {ac }}$ (except in the proof of Corollary 1.20), with the ring of integers $O_{F}$, and the absolute Galois group $\Gamma_{F}:=\operatorname{Gal}\left(\mathbb{Q}^{\text {ac }} / F\right) \subset \Gamma_{\mathbb{Q}}$. Denote by $\theta$ the non-trivial involution in $\operatorname{Gal}(F / \mathbb{Q})$ or its local avatar.

1.1.1. Motives and L-functions. We consider an elliptic curve $E$ over $\mathbb{Q}$ of conductor $N$. For each prime $p$, denote by $\mathrm{T}_{p}(E)$ the $p$-adic Tate module $\lim _{n} E\left[p^{n}\right]\left(\mathbb{Q}^{\text {ac }}\right)$ of $E$, which is equipped with a $\mathbb{Z}_{p}$-linear continuous action of $\Gamma_{\mathbb{Q}}$. Put $\overleftarrow{\mathrm{V}_{p}(E)}=\mathrm{T}_{p}(E) \otimes_{\mathbb{Z}_{p}} \mathbb{Q}_{p}$. 
Let $\sigma$ be the irreducible cuspidal automorphic representation of $\mathrm{GL}_{2}(\mathbb{A})$ associated to $E$, whose existence is guaranteed by the famous works on the modularity of rational elliptic curves in [Wi195, TW95, BCDT01].

We consider another elliptic curve $A$ over $F$ whose conductor has norm $M$. Put $A^{\theta}=$ $A \otimes_{F, \theta} F$. For each prime $p$, we similarly define $\mathrm{V}_{p}(A)$, which is a $p$-adic representation of $\Gamma_{F}$. The tensor product representation $\mathrm{V}_{p}(A) \otimes_{\mathbb{Q}_{p}} \mathrm{~V}_{p}\left(A^{\theta}\right)$ has a natural extension to the larger group $\Gamma_{\mathbb{Q}}$, known as the Asai representation, denoted by $\operatorname{As~}_{p}(A)$. Let $\pi$ be the irreducible cuspidal automorphic representation of $\operatorname{Res}_{F / \mathbb{Q}} \mathrm{GL}_{2}(\mathbb{A})$ associated to $A$, whose existence is guaranteed by the very recent works of Le Hung [LH14] and Freitas-Le Hung-Siksek [FLHS] on the modularity of elliptic curves over real quadratic fields.

Similarly to the Galois representation, the $F$-motive $\mathrm{h}^{1}(A) \otimes \mathrm{h}^{1}\left(A^{\theta}\right)$ has a natural descent to a $\mathbb{Q}$-motive, known as the Asai motive, denoted by $\operatorname{Ash}^{1}(A)$, whose $p$-adic realization is the Galois representation $\operatorname{As}\left(\mathrm{V}_{p}(A)(-1)\right)$. We define the $\mathbb{Q}$-motive

$$
\mathrm{M}_{E, A}=\mathrm{h}^{1}(E)(1) \otimes \operatorname{Ash}^{1}(A)(1) .
$$

In particular, its $p$-adic realization $\left(\mathrm{M}_{E, A}\right)_{p}$ is $\mathrm{V}_{p}(E) \otimes_{\mathbb{Q}_{p}}\left(\mathrm{As}_{p}(A)\right)(-1)$ whose weight is -1. The motive $\mathrm{M}_{E, A}$ is equipped with a canonical polarization $\mathrm{M}_{E, A} \stackrel{\sim}{\rightarrow} \mathrm{M}_{E, A}^{\vee}(1)$ from the Poincaré duality pairing $\mathrm{M}_{E, A} \times \mathrm{M}_{E, A} \rightarrow \mathbb{Q}(1)$. The polarization is of symplectic type.

The $L$-function $L\left(s, \mathrm{M}_{E, A}\right)$ associated to the motive $\mathrm{M}_{E, A}$ satisfies the relation

$$
L\left(s, \mathrm{M}_{E, A}\right)=L(s+1 / 2, \sigma \times \pi),
$$

where the latter is the (twisted) triple product $L$-function, which is the same as the tensor product $L$-function of $\sigma \times$ As $\pi$. By the work of Garrett [Gar87] and PiatetskiShapiro-Rallis [PSR87], it has a meromorphic continuation to the entire complex plane and is holomorphic at $s=0$, satisfying the following functional equation

$$
L\left(s, \mathrm{M}_{E, A}\right)=\epsilon\left(\mathrm{M}_{E, A}\right) c\left(\mathrm{M}_{E, A}\right)^{-s} L\left(-s, \mathbf{M}_{E, A}\right)
$$

for the root number $\epsilon\left(\mathrm{M}_{E, A}\right) \in\{ \pm 1\}$ and some positive integer $c\left(\mathrm{M}_{E, A}\right)$.

Consider the following assumptions.

Assumption 1.1 (Group E). We assume that

(E1): Neither $E$ nor $A$ has complex multiplication over $\mathbb{Q}^{\text {ac }}$;

(E2): $N$ and $M \operatorname{disc}(F)$ are coprime;

(E3): If we write $N=N^{+} N^{-}$such that $N^{+}$(resp. $N^{-}$) is the product of prime factors that are split (resp. inert) in $F$, then $N^{-}$is square-free.

Definition 1.2. Denote by $\wp\left(N^{-}\right) \geq 0$ the number of distinct prime factors of $N^{-}$. We say that the pair $(E, A)$ is of even (resp. odd) type if Assumption 1.1 is satisfied and $\wp\left(N^{-}\right)$is odd (resp. even).

By the result of Prasad [Pra92, Theorems B \& D, Remark 4.1.1], we have $\epsilon\left(\mathrm{M}_{E, A}\right)=$ $(-1)^{\wp\left(N^{-}\right)+1}$ for $(E, A)$ satisfying Assumption 1.1. In particular, if $(E, A)$ is of odd type, then we have $L\left(0, \mathrm{M}_{E, A}\right)=0$. 
Theorem 1.3. Suppose that $(E, A)$ satisfy Assumption 1.1. If $L\left(0, \mathrm{M}_{E, A}\right) \neq 0$, which in particular implies that $(E, A)$ is of even type, then for all but finitely many primes $p$, we have

$$
\operatorname{dim}_{\mathbb{Q}_{p}} \mathrm{H}_{f}^{1}\left(\mathbb{Q},\left(\mathrm{M}_{E, A}\right)_{p}\right)=0 .
$$

From the above theorem, we can deduce the following one, whose statement has nothing to do with the quadratic field $F$.

Theorem 1.4. Let $E_{1}$ and $E_{2}$ be two rational elliptic curves of conductors $N_{1}$ and $N_{2}$, respectively. Suppose that $N_{1}$ and $N_{2}$ are coprime; $E_{1}$ has multiplicative reduction at at least one finite place; and $E_{2}$ has no complex multiplication over $\mathbb{Q}^{\text {ac }}$. If the central critical value $L\left(2, E_{1} \times \operatorname{Sym}^{2} E_{2}\right) \neq 0$, then for all but finitely many primes $p$, we have

$$
\operatorname{dim}_{\mathbb{Q}_{p}} \mathrm{H}_{f}^{1}\left(\mathbb{Q}, \mathrm{V}_{p}\left(E_{1}\right) \otimes_{\mathbb{Q}_{p}}\left(\operatorname{Sym}^{2} \mathrm{~V}_{p}\left(E_{2}\right)\right)(-1)\right)=0 .
$$

We will give the proof in $§ 1.3$, actually for its equivalent form Corollary 1.20.

1.1.2. A candidate in the Selmer group. Let $X=X_{N^{+} M}$ be the minimal resolution of the Baily-Borel compactification of the Hilbert modular surface associated to $F$ of level- $N^{+} M$ structure, which is a smooth projective surface over $\mathbb{Q}$ (see $\S 2.4$ for details). In this article, our level structures are always of $\Gamma_{0}$-type in the classical sense, unless otherwise specified.

Suppose that $(E, A)$ is of odd type. Let $B_{N^{-}}$be the indefinite quaternion algebra over $\mathbb{Q}$, ramified exactly at primes dividing $N^{-}$, which exists since $\wp\left(N^{-}\right)$is even. Denote by $Y=Y_{N^{+} M, N^{-}}$the (compactified) Shimura curve associated to $B_{N^{-}}$of level- $N^{+} M$ structure. By modular interpretation, we have a natural finite morphism $\zeta: Y \rightarrow X$ (see §2.5), which was first studied by Hirzebruch-Zagier in this context. Put $Z=$ $Y \times_{\text {Spec } \mathbb{Q}} X$. Then the graph of $\zeta$ defines a Chow cycle $\Delta$ in $\mathrm{CH}^{2}(Z)$, which we call a Hirzebruch-Zagier cycle. This cycle is analogous to the Gross-Kudla-Schoen cycle (see [GS95] and [GK92]) on triple product of (modular) curves, and is an example of special cycles on Shimura varieties in general.

We denote by $\mathrm{H}_{\sigma}^{1}\left(Y_{\mathbb{Q}^{a c}}\right)$ (resp. $\mathrm{H}_{\pi}^{2}\left(X_{\mathbb{Q}^{a c}}\right)$ ) the $\sigma$-isotypic (resp. $\pi$-isotypic) subspace of $\mathrm{H}_{\text {êt }}^{1}\left(Y_{\mathbb{Q}^{\text {acc }}}, \mathbb{Q}_{p}(1)\right)$ (resp. $\left.\mathrm{H}_{\text {êt }}^{2}\left(X_{\mathbb{Q}^{\mathrm{ac}}}, \mathbb{Q}_{p}(1)\right)\right)$ under the Hecke action. Then it is a direct summand as a $p$-adic representation of $\Gamma_{\mathbb{Q}}$. Choose a Hecke projector $\mathscr{P}_{\sigma, \pi} \in \mathrm{CH}^{3}\left(Z \times_{\text {Spec } \mathbb{Q}} Z\right) \otimes_{\mathbb{Z}} \mathbb{Q}$ for $(\sigma, \pi)$, viewed as a Chow correspondence (see $\S 3.3 .3$ for details). We have the Chow cycle

$$
\Delta_{E, A}:=\mathscr{P}_{\sigma, \pi}^{*} \Delta \in \mathrm{CH}^{2}(Z) \otimes_{\mathbb{Z}} \mathbb{Q},
$$

which has the trivial image under the geometric cycle class map $\mathrm{cl}_{Z}^{0}: \mathrm{CH}^{2}(Z) \otimes_{\mathbb{Z}} \mathbb{Q} \rightarrow$ $\mathrm{H}_{\text {êt }}^{4}\left(Z_{\mathbb{Q}^{a c}}, \mathbb{Q}_{p}(2)\right)$. Therefore, it induces an element

$$
{ }^{p} \Delta_{E, A}:=\operatorname{AJ}_{p}\left(\Delta_{E, A}\right) \in \mathrm{H}^{1}\left(\mathbb{Q}, \mathrm{H}_{\text {ét }}^{3}\left(Z_{\mathbb{Q}^{\text {ac }}}, \mathbb{Q}_{p}(2)\right)\right)
$$

via the (p-adic) étale Abel-Jacobi map $\mathrm{AJ}_{p}$. As we will see in Proposition 3.15, the element ${ }^{p} \Delta_{E, A}$ does not depend on the choice of $\mathscr{P}_{\sigma, \pi}$, and moreover belongs to the Bloch-Kato Selmer group $\mathrm{H}_{f}^{1}\left(\mathbb{Q}, \mathrm{H}_{\sigma}^{1}\left(Y_{\mathbb{Q}^{\mathrm{ac}}}\right) \otimes_{\mathbb{Q}_{p}} \mathrm{H}_{\pi}^{2}\left(X_{\mathbb{Q}^{\mathrm{ac}}}\right)\right)$; see $\S 3.1$ for the general definition. Note that by Conjecture 1.9, even the Chow cycle $\Delta_{E, A}$ itself is independent of the choice of $\mathscr{P}_{\sigma, \pi}$. 
It is well-known that as $p$-adic representations of $\Gamma_{\mathbb{Q}}$, we have $\mathrm{H}_{\sigma}^{1}\left(Y_{\mathbb{Q}^{\mathrm{ac}}}\right) \simeq \mathrm{V}_{p}(E)^{\oplus b}$ for some $b \geq 1$. We will show that the $p$-adic representation $\mathrm{H}_{\pi}^{2}\left(X_{\mathbb{Q}^{\text {ac }}}\right)$ of $\Gamma_{\mathbb{Q}}$ is isomorphic to $\left(\operatorname{As~}_{p}(A)\right)(-1)^{\oplus a}$ for some $a \geq 1$. In particular, the class ${ }^{p} \Delta_{E, A}$ may be regarded as an element in

$$
\mathrm{H}_{f}^{1}\left(\mathbb{Q}, \mathrm{V}_{p}(E) \otimes_{\mathbb{Q}_{p}}\left(\mathrm{As} \mathrm{V}_{p}(A)\right)(-1)\right)^{\oplus a b}=\mathrm{H}_{f}^{1}\left(\mathbb{Q},\left(\mathrm{M}_{E, A}\right)_{p}\right)^{\oplus a b}
$$

Theorem 1.5. Suppose that $(E, A)$ is of odd type. For all but finitely many primes $p$, if ${ }^{p} \Delta_{E, A} \neq 0$, then

$$
\operatorname{dim}_{\mathbb{Q}_{p}} \mathrm{H}_{f}^{1}\left(\mathbb{Q},\left(\mathrm{M}_{E, A}\right)_{p}\right)=1 .
$$

The reader may consult $\S 1.2 .2$ to see why we consider the Chow cycle $\Delta_{E, A}$.

Remark 1.6.

(1) In Theorems 1.3, 1.4 and 1.5, we have explicit conditions on $p$ for which those theorems hold; see Definition 5.18.

(2) The Galois representation $\mathrm{As}_{p}(A)$, and hence $\left(\mathrm{M}_{E, A}\right)_{p}$, are decomposable if and only if $A^{\theta}$ is isogenous to a (possibly trivial) quadratic twist of $A$. We will take a closer look at a special case where $A^{\theta}$ is isogenous to $A$ in $\S 1.3$.

(3) Assumption 1.1 (E2, E3) are not essentially necessary. For each place $v$ of $\mathbb{Q}$, denote by $\epsilon\left(1 / 2, \sigma_{v} \times \pi_{v}\right) \in\{ \pm 1\}$ the (twisted) triple product $\epsilon$-factor. Put

$$
\Sigma_{\sigma, \pi}=\left\{v<\infty \mid \epsilon\left(1 / 2, \sigma_{v} \times \pi_{v}\right) \eta_{v}(-1)=-1\right\},
$$

where $\eta_{v}: \mathbb{Q}_{v}^{\times} \rightarrow\{ \pm 1\}$ is the character associated to $F_{v} / \mathbb{Q}_{v}$. Our method should be applicable under the only requirement that $v \| N$ if $v \in \Sigma_{\sigma, \pi}$ (which already implies $v \mid N$ ), and moreover be able to prove Theorems 1.3, 1.4 and 1.5 for all primes $p$. But these may tremendously increase the technical complication of the proof, and hence we decide not to proceed with that generality.

Remark 1.7. The idea of exploiting diagonal special cycles in Euler System-style arguments originated in the work of Darmon and Rotger [DR14,DRII], who exploited them to obtain a result for the motive of an elliptic curve twisted by two 2-dimensional Artin representations, which is also of rank 8. They showed that the Mordell-Weil group (as a twisted version of the classical Mordell-Weil group of an elliptic curve) is finite if the corresponding $L$-function has non-vanishing central value. Both their work and ours provide evidence of the Beilinson-Bloch conjecture and the Bloch-Kato conjecture, for the pair $\mathrm{SO}(3) \times \mathrm{SO}(4)$ in the framework of Gan-Gross-Prasad, both using diagonal special cycles. But there are some differences:

- They deal with the split case (in particular, the geometric object is a triple product of modular curves), while we deal with the quasi-split case.

- The (range of) Hodge-Tate weight of their motive is 1, while ours is 3 as the motive involves two elliptic curves.

- The method they use is $p$-adic, especially via Hida families, while ours is more geometric by exploiting "tame" deformations at auxiliary primes $\ell$ not equal to $p$ in bounding the pro- $p$-Selmer group.

- More importantly, in the rank-0 case, our proof actually indicates that the $p^{\infty}$ part of the Tate-Shafarevich group of the motive, defined in a suitable way, is 
trivial for all but finitely many primes $p$; while their method is only able to control the image of the pro- $p$-Selmer group in the local cohomology at $p$, from which it seems very hard to deduce bounds on the relevant Tate-Shafarevich groups in settings other than Mordell-Weil groups.

1.2. Connection with $L$-function and Beilinson-Bloch-Kato conjecture. For a number field $K$, denote by $\operatorname{Mot}_{K}^{\text {rat }}$ the pseudo-abelian category of Chow motives over $K$ with coefficient $\mathbb{Q}$ (see [Sch94] for example). For a Chow motive $\mathbf{M}$, we have the $p$-adic realization $\mathrm{M}_{p}$, which is a $p$-adic representation of $\operatorname{Gal}\left(K^{\mathrm{ac}} / K\right)$, for each prime $p$. Recall that for each smooth projective scheme $X$ over $K$ purely of dimension $d$, we have the Picard motive $\mathrm{h}^{2 d-1}(X)$ and the Albanese motive $\mathrm{h}^{1}(X)$. For each Chow motive $\mathrm{M}$, we have an associated $L$-function $L(s, \mathrm{M})$ which is defined for $\operatorname{Re} s$ sufficiently large. A polarization of $\mathrm{M}$ is an isomorphism $\mathrm{M} \stackrel{\sim}{\rightarrow} \mathrm{M}^{\vee}(1)$ in the category $\operatorname{Mot}_{K}^{\text {rat }}$.

\subsubsection{Beilinson-Bloch / Bloch-Kato conjecture.}

Definition 1.8. Let $\mathrm{M}$ be a Chow motive. We define the Chow group of $\mathrm{M}$ (of degree 0) to be

$$
\mathrm{CH}(\mathrm{M})=\operatorname{Hom}_{\mathrm{Mot}_{K}^{\mathrm{rat}}}\left(\mathbb{1}_{K}, \mathrm{M}\right)
$$

where $\mathbb{1}_{K}$ is the unit motive over $K$.

Denote by $\mathrm{CH}(\mathrm{M})^{0}$ the kernel of the geometric cycle class map

$$
\mathrm{cl}_{\mathrm{M}}^{0}: \mathrm{CH}(\mathrm{M}) \rightarrow \mathrm{H}^{0}\left(K, \mathrm{M}_{p}\right)
$$

for some prime $p$, which is known to be independent of $p$. We have the following (p-adic) étale Abel-Jacobi map

$$
\mathrm{AJ}_{p}: \mathrm{CH}(\mathrm{M})^{0} \rightarrow \mathrm{H}^{1}\left(K, \mathrm{M}_{p}\right)
$$

for each prime $p$. The following conjecture is a part of the Beilinson-Bloch conjecture; see for example [Jan90, 9.15].

Conjecture 1.9. Let notation be as above. The étale Abel-Jacobi map $\mathrm{AJ}_{p}$ is injective for every prime $p$.

The following conjecture is a combination of the Beilinson-Bloch conjecture and the Bloch-Kato conjecture.

Conjecture 1.10. Let $\mathrm{M}$ be a polarized Chow motive in $\operatorname{Mot}_{K}^{\mathrm{rat}}$.

(1) The $\mathbb{Q}_{p}$-linearization

$$
\mathrm{AJ}_{p}: \mathrm{CH}(\mathrm{M})^{0} \otimes_{\mathbb{Q}} \mathbb{Q}_{p} \rightarrow \mathrm{H}^{1}\left(K, \mathrm{M}_{p}\right)
$$

is an isomorphism onto the Bloch-Kato Selmer group $\mathrm{H}_{f}^{1}\left(K, \mathrm{M}_{p}\right)$, which is a subspace of $\mathrm{H}^{1}\left(K, \mathrm{M}_{p}\right)$, for all $p$.

(2) The L-function $L(s, \mathrm{M})$ associated to $\mathrm{M}$ has a meromorphic continuation to the entire complex plane, satisfying the following functional equation

$$
L(s, \mathrm{M})=\epsilon(\mathrm{M}) c(\mathbf{M})^{-s} L(-s, \mathrm{M})
$$

for the root number $\epsilon(\mathrm{M}) \in\{ \pm 1\}$ and some positive integer $c(\mathrm{M})$. 
(3) For all $p$, we have

$$
\operatorname{ord}_{s=0} L(s, \mathrm{M})=\operatorname{dim}_{\mathbb{Q}_{p}} \mathrm{H}_{f}^{1}\left(K, \mathrm{M}_{p}\right)-\operatorname{dim}_{\mathbb{Q}_{p}} \mathrm{H}^{0}\left(K, \mathrm{M}_{p}\right) .
$$

Recall from $\S 1.1$ that we have the surface $X$, and when $(E, A)$ is of odd type, the curve $Y$ and hence the three-fold $Z=Y \times_{\text {Spec } \mathbb{Q}} X$, all being smooth projective over $\mathbb{Q}$. We have the Chow motive $\mathrm{h}^{2}(X)$, defined uniquely up to isomorphism, by Murre [Mur90]. The motive $\mathrm{h}^{1}(Y)(1) \otimes \mathrm{h}^{2}(X)(1)$ is canonically polarized by the Poincaré duality.

Conjecture 1.11. The motive $\operatorname{Ash}^{1}(A)$ is a direct summand of $\mathrm{h}^{2}(X)$ in the pseudoabelian category $\operatorname{Mot}_{\mathbb{Q}}^{\mathrm{rat}}$.

Note that for our motive $\mathrm{M}_{E, A}$, we have $\mathrm{H}^{0}\left(\mathbb{Q},\left(\mathrm{M}_{E, A}\right)_{p}\right)=0$ for all $p$ and hence $\mathrm{CH}\left(\mathrm{M}_{E, A}\right)^{0}=\mathrm{CH}\left(\mathrm{M}_{E, A}\right)$. The following is a consequence of (1.1), Theorems 1.3 and 1.5 .

Corollary 1.12. Let $(E, A)$ be a pair satisfying Assumption 1.1.

(1) Conjecture 1.10 (2) holds for $\mathrm{M}_{E, A}$.

(2) Suppose that $\operatorname{ord}_{s=0} L\left(s, \mathrm{M}_{E, A}\right)=0$. Then Conjecture 1.10 (3) holds for $\mathrm{M}_{E, A}$ and all but finitely many $p$.

(3) Suppose that the pair $(E, A)$ is of odd type. We further assume Conjecture 1.9 for $\mathrm{h}^{1}(Y)(1) \otimes \mathrm{h}^{2}(X)(1)$, and Conjecture 1.11. If $\Delta_{E, A} \neq 0$, then Conjecture 1.10 (1) holds for $\mathrm{M}_{E, A}$ and all but finitely many primes $p$.

Remark 1.13. Since currently we do not know Conjecture 1.9 for $\mathrm{h}^{1}(Y)(1) \otimes \mathrm{h}^{2}(X)(1)$, it is not clear that ${ }^{p} \Delta_{E, A}=\mathrm{AJ}_{p}\left(\Delta_{E, A}\right) \neq 0$ for one prime $p$ implies the same for others. We also do not even know if $\operatorname{dim}_{\mathbb{Q}_{p}} \mathrm{H}_{f}^{1}\left(\mathbb{Q},\left(\mathrm{M}_{E, A}\right)_{p}\right)$ are same for all (but finitely many) p. However, see Corollary 1.19.

1.2.2. Arithmetic Gan-Gross-Prasad conjecture. The Chow cycle $\Delta_{E, A}$ is closely related to the Gross-Prasad conjecture [GP92]. In fact, the product $\sigma \times \pi$ can be viewed as a cuspidal automorphic representation of $H \times G$, where $H \simeq \mathrm{SO}(3)$ is the split special orthogonal group over $\mathbb{Q}$ of $\operatorname{rank} 3$ and $G \simeq \mathrm{SO}(4)$ is the quasi-split special orthogonal group over $\mathbb{Q}$ of rank 4 and with discriminant field $F$. The global (Gan-)Gross-Prasad conjecture [GGP12] predicts that the central value $L(1 / 2, \sigma \times \pi)$ is non-vanishing if and only if there is a non-trivial diagonal automorphic period integral for some member $\sigma^{\prime} \times \pi^{\prime}$, as an automorphic representation of some pure inner form $H^{\prime} \times G^{\prime}$ of $H \times G$, in the Vogan packet of $\sigma \times \pi$. If such $\sigma^{\prime} \times \pi^{\prime}$ exists, then it is unique, and the group $H^{\prime} \times G^{\prime}$ is determined by all local epsilon factors $\epsilon\left(1 / 2, \sigma_{v} \times \pi_{v}\right) \in\{ \pm 1\}$. Denote by $\Sigma_{\sigma, \pi}$ the (finite) set of places $v$ of $\mathbb{Q}$ such that $\epsilon\left(1 / 2, \sigma_{v} \times \pi_{v}\right) \eta_{v}(-1)=-1$, where $\eta_{v}: \mathbb{Q}_{v}^{\times} \rightarrow\{ \pm 1\}$ is the character associated to $F_{v} / \mathbb{Q}_{v}$.

In our case, the set $\Sigma_{\sigma, \pi}$ consists of $\infty$ and all prime factors of $N^{-}$, by [Pra92, Theorems B \& D, Remark 4.1.1]. In particular, it has even (resp. odd) cardinality if $(E, A)$ is of even (resp. odd) type. Suppose that $(E, A)$ is of odd type, which implies that the (global) root number $\epsilon\left(\mathrm{M}_{E, A}\right)=\epsilon(1 / 2, \sigma \times \pi)=-1$ and $\operatorname{ord}_{s=0} L\left(s, \mathrm{M}_{E, A}\right)$ is odd by (1.1). This is usually referred as the arithmetic case. As a consequence, the central 
$L$-value $L\left(0, \mathrm{M}_{E, A}\right)=L(1 / 2, \sigma \times \pi)$ vanishes. Moreover, all diagonal automorphic period integrals for the entire Vogan packet of $\sigma \times \pi$ vanish simply by the local epsilon dichotomy [Pra92, Theorems B \& D]. In the arithmetic case, we consider (modified) diagonal cycles instead of diagonal period integrals. The following conjecture can be regarded as an arithmetic version of the global Gan-Gross-Prasad conjecture.

Conjecture 1.14 (Arithmetic GGP for $\mathrm{SO}(3) \times \mathrm{SO}(4)$, unrefined version). Suppose that $(E, A)$ is of odd type. Then the Chow cycle $\Delta_{E, A} \neq 0$ if and only if $L^{\prime}\left(0, \mathrm{M}_{E, A}\right) \neq 0$.

Remark 1.15. There is no local obstruction for $\Delta_{E, A}$ being non-zero. In fact, the local invariant functionals [Pra92] can be realized at the level structure defining the Shimura varieties $Y$ and $X$, as told by Lemma 3.21.

In the case where the group $G$ is the split special orthogonal group over $\mathbb{Q}$ of rank 4 , we can construct a similar Chow motive $\mathrm{M}$ but from three elliptic curves defined over $\mathbb{Q}$. Then the above conjecture was proposed by Gross and Kudla [GK92], in terms of the Beilinson-Bloch height. Later, Gross and Keating [GK93] did crucial local computation toward this conjecture, actually applicable to both the split case and the quasi-split case as in Conjecture 1.14. In a recent preprint [YZZ], X. Yuan, S. Zhang and W. Zhang made important progress toward the global height formula in the split case under certain conditions. On the other hand, in [Zha12], the author proposed the Arithmetic Gan-Gross-Prasad conjecture for close pairs of unitary groups of arbitrary ranks, together with the approach via relative trace formulae, and obtained some local results as evidence toward the conjecture.

In view of the Arithmetic Gan-Gross-Prasad conjecture, Theorem 1.5 has the following corollary.

Corollary 1.16. Let $(E, A)$ be of odd type such that $\operatorname{ord}_{s=0} L\left(s, \mathrm{M}_{E, A}\right)=1$. If we assume Conjecture 1.9 for the motive $\mathrm{h}^{1}(Y)(1) \otimes \mathrm{h}^{2}(X)(1)$, and Conjecture 1.14, then Conjecture 1.10 (3) holds for $\mathrm{M}_{E, A}$ and all but finitely many primes $p$.

In view of Conjectures 1.10 and 1.14, we propose the following.

Conjecture 1.17. Let $(E, A)$ be an arbitrary pair.

(1) Suppose that either one of the following holds

- $\operatorname{dim}_{\mathbb{Q}_{p}} \mathrm{H}_{f}^{1}\left(\mathbb{Q},\left(\mathrm{M}_{E, A}\right)_{p}\right)=0$ for some prime $p$;

- $\operatorname{dim}_{\mathbb{Q}} \mathrm{CH}\left(\mathrm{M}_{E, A}\right)=0$;

- $\operatorname{ord}_{s=0} L\left(s, \mathrm{M}_{E, A}\right)=0$.

Then for all primes $p$, we have

$$
\operatorname{dim}_{\mathbb{Q}} \mathrm{CH}\left(\mathrm{M}_{E, A}\right)=\operatorname{ord}_{s=0} L\left(s, \mathrm{M}_{E, A}\right)=\operatorname{dim}_{\mathbb{Q}_{p}} \mathrm{H}_{f}^{1}\left(\mathbb{Q},\left(\mathrm{M}_{E, A}\right)_{p}\right)=0 .
$$

(2) Suppose that either one of the following holds

- $\operatorname{dim}_{\mathbb{Q}_{p}} \mathrm{H}_{f}^{1}\left(\mathbb{Q},\left(\mathrm{M}_{E, A}\right)_{p}\right)=1$ for some $p$;

- $\operatorname{dim}_{\mathbb{Q}} \mathrm{CH}\left(\mathrm{M}_{E, A}\right)=1$;

- $\operatorname{ord}_{s=0} L\left(s, \mathrm{M}_{E, A}\right)=1$.

Then $\Delta_{E, A} \neq 0$, and for all primes $p$, we have

$$
\operatorname{dim}_{\mathbb{Q}} \mathrm{CH}\left(\mathrm{M}_{E, A}\right)=\operatorname{ord}_{s=0} L\left(s, \mathrm{M}_{E, A}\right)=\operatorname{dim}_{\mathbb{Q}_{p}} \mathrm{H}_{f}^{1}\left(\mathbb{Q},\left(\mathrm{M}_{E, A}\right)_{p}\right)=1 .
$$


Here, the class $\Delta_{E, A}$ may be constructed via a similar way.

1.3. The case of base change. Let us consider a special but illustrative case where $A$ is isogenous to $A^{b} \otimes_{\mathbb{Q}} F$ for an elliptic curve $A^{b}$ over $\mathbb{Q}$. This immediately implies that $A^{\theta}$ is isogenous to $A$, but the latter is slightly weaker. In this case, we have a decomposition

$$
\left(\operatorname{As~}_{p}(A)\right)(-1)=\left(\operatorname{Sym}^{2} \mathrm{~V}_{p}\left(A^{b}\right)\right)(-1) \oplus \mathbb{Q}_{p}(\eta)
$$

of $p$-adic representations of $\Gamma_{\mathbb{Q}}$, where $\eta: \Gamma_{\mathbb{Q}} \rightarrow\{ \pm 1\}$ is the quadratic character associated to $F / \mathbb{Q}$ via global class field theory. Note that $\mathrm{V}_{p}(E) \otimes_{\mathbb{Q}_{p}} \mathbb{Q}_{p}(\eta) \simeq \mathrm{V}_{p}\left(E^{F}\right)$, where $E^{F}$ is the $F$-twist of $E$.

Suppose that $(E, A)$ is of odd type. Denote by ${ }^{p} \Delta_{E, A^{b}}$ (resp. ${ }^{p} \Delta_{E^{F}}$ ) the projection of ${ }^{p} \Delta_{E, A}$ into $\mathrm{H}_{f}^{1}\left(\mathbb{Q}, \mathrm{V}_{p}(E) \otimes_{\mathbb{Q}_{p}}\left(\operatorname{Sym}^{2} \mathrm{~V}_{p}\left(A^{b}\right)\right)(-1)\right)^{\oplus a b}\left(\operatorname{resp} . \mathrm{H}_{f}^{1}\left(\mathbb{Q}, \mathrm{V}_{p}\left(E^{F}\right)\right)^{\oplus a b}\right)$. Then Theorem 1.5 asserts that for all but finitely many primes $p$, there is at most one member between ${ }^{p} \Delta_{E, A^{b}}$ and ${ }^{p} \Delta_{E^{F}}$ that could be non-zero, and moreover

(1) if ${ }^{p} \Delta_{E, A^{b}} \neq 0$, then

$$
\operatorname{dim}_{\mathbb{Q}_{p}} \mathrm{H}_{f}^{1}\left(\mathbb{Q}, \mathrm{V}_{p}(E) \otimes_{\mathbb{Q}_{p}}\left(\operatorname{Sym}^{2} \mathrm{~V}_{p}\left(A^{b}\right)\right)(-1)\right)=1, \quad \operatorname{dim}_{\mathbb{Q}_{p}} \mathrm{H}_{f}^{1}\left(\mathbb{Q}, \mathrm{V}_{p}\left(E^{F}\right)\right)=0 ;
$$

(2) if ${ }^{p} \Delta_{E^{F}} \neq 0$, then

$$
\operatorname{dim}_{\mathbb{Q}_{p}} \mathrm{H}_{f}^{1}\left(\mathbb{Q}, \mathrm{V}_{p}(E) \otimes_{\mathbb{Q}_{p}}\left(\operatorname{Sym}^{2} \mathrm{~V}_{p}\left(A^{b}\right)\right)(-1)\right)=0, \quad \operatorname{dim}_{\mathbb{Q}_{p}} \mathrm{H}_{f}^{1}\left(\mathbb{Q}, \mathrm{V}_{p}\left(E^{F}\right)\right)=1 .
$$

Suppose that ${ }^{p} \Delta_{E^{F}} \neq 0$. By the main theorem of [Ski14] or [Zha14], the rank of $E^{F}(\mathbb{Q})$ is 1 , or equivalently, the rank of $E(F)^{\theta=-1}$ is 1 . A natural question would be: How to construct a $\mathbb{Q}$-generator of $E(F)^{\theta=-1}$, from the information that ${ }^{p} \Delta_{E^{F}} \neq 0$ ?

Note that we have the geometric cycle class map

$$
\mathrm{cl}_{X_{F}}^{0}: \mathrm{CH}^{1}\left(X_{F}\right) \otimes_{\mathbb{Z}} \mathbb{Q} \rightarrow \mathrm{H}_{\text {ét }}^{2}\left(X_{\mathbb{Q}^{\mathrm{ac}}}, \mathbb{Q}_{p}(1)\right)^{\Gamma_{F}} .
$$

By the Tate conjecture for the surface $X_{F}$ in the version proved in [HLR86], we have

$$
\left(\operatorname{Im}\left(\mathrm{cl}_{X_{F}}^{0}\right) \cap \mathrm{H}_{\pi}^{2}\left(X_{\mathbb{Q}^{\mathrm{ac}}}\right)\right) \otimes_{\mathbb{Z}} \mathbb{Q}_{p}=\mathrm{H}_{\pi}^{2}\left(X_{\mathbb{Q}^{\mathrm{ac}}}\right)^{\Gamma_{F}} \simeq \mathbb{Q}_{p}^{\oplus a} .
$$

Let $\mathrm{CH}_{\pi}^{1}\left(X_{F}\right)$ be the subspace of $\mathrm{CH}^{1}\left(X_{F}\right) \otimes_{\mathbb{Z}} \mathbb{Q}$ whose image under $\mathrm{cl}_{X_{F}}^{0}$ is contained in $\mathrm{H}_{\pi}^{2}\left(X_{\mathbb{Q}^{\mathrm{ac}}}\right)$. If we regard $\Delta_{E, A}$ as a correspondence from $Y$ to $X$, then $\phi_{*} \Delta_{E, A}^{*} \mathrm{CH}_{\pi}^{1}\left(X_{F}\right)$ is contained in $\left(E(F) \otimes_{\mathbb{Z}} \mathbb{Q}\right)^{\theta=-1}$ for every modular parametrization $\phi: Y \rightarrow E$. One can check that ${ }^{p} \Delta_{E^{F}} \neq 0$ implies the existence of $\phi$ such that $\phi_{*} \Delta_{E, A}^{*} \mathrm{CH}_{\pi}^{1}\left(X_{F}\right)$ is non-zero. Combining with Theorem 1.5, we have

$$
\phi_{*} \Delta_{E, A}^{*} \mathrm{CH}_{\pi}^{1}\left(X_{F}\right)=\left(E(F) \otimes_{\mathbb{Z}} \mathbb{Q}\right)^{\theta=-1}=E^{F}(\mathbb{Q}) \otimes_{\mathbb{Z}} \mathbb{Q},
$$

which has dimension 1 .

Remark 1.18. The above discussion is logically unhelpful for the proof of Theorem 1.5, since it is very special that $A$ comes from the base change. Nevertheless, this observation, on the contrary, is a starting point of our strategy for the proofs of both Theorem 1.5 and Theorem 1.3: The key theorems - Theorems 4.10 and 4.11, which are explicit congruence formulae for various Hirzebruch-Zagier classes, involve computation of their localizations at sophisticatedly chosen primes. We realize such computation as 
an application of the Tate conjecture for Hilbert modular surfaces over finite fields, together with some geometric arguments that are very close to the above pullback along correspondences.

The following corollary is a natural consequence of Theorem 1.5 and the above discussion, since we know Conjecture 1.9 for the motive $\mathrm{h}^{1}\left(E^{F}\right)(1)$.

Corollary 1.19. Suppose that the pair $(E, A)$ is of odd type, and $A$ is isogenous to $A^{b} \otimes_{\mathbb{Q}} F$ for an elliptic curve $A^{b}$ over $\mathbb{Q}$. If ${ }^{p} \Delta_{E^{F}}$ is non-zero for some $p$, then ${ }^{p} \Delta_{E^{F}}$ and hence ${ }^{p} \Delta_{E, A}$ are non-zero for every $p$. Moreover, we have $\operatorname{dim}_{\mathbb{Q}_{p}} \mathrm{H}_{f}^{1}\left(\mathbb{Q},\left(\mathrm{M}_{E, A}\right)_{p}\right)=1$ for all but finitely many $p$.

We also have the following result, which is a corollary of Theorem 1.3 and is equivalent to Theorem 1.4. It provides evidence of Conjecture 1.10 for the motive $\mathrm{h}^{1}\left(E_{1}\right)(1) \otimes\left(\operatorname{Sym}^{2} \mathrm{~h}^{1}\left(E_{2}\right)\right)(1)$ over $\mathbb{Q}$ of rank 6 , which is also canonically polarized of symplectic type.

Corollary 1.20. Let $E_{1}$ and $E_{2}$ be two rational elliptic curves of conductors $N_{1}$ and $N_{2}$, respectively. Suppose that $N_{1}$ and $N_{2}$ are coprime; $E_{1}$ has multiplicative reduction at at least one finite place; and $E_{2}$ has no complex multiplication over $\mathbb{Q}^{\text {ac }}$. If the central critical value $L\left(0, \mathrm{~h}^{1}\left(E_{1}\right)(1) \otimes\left(\operatorname{Sym}^{2} \mathrm{~h}^{1}\left(E_{2}\right)\right)(1)\right) \neq 0$, then for all but finitely many primes $p$, we have

$$
\operatorname{dim}_{\mathbb{Q}_{p}} \mathrm{H}_{f}^{1}\left(\mathbb{Q}, \mathrm{V}_{p}\left(E_{1}\right) \otimes_{\mathbb{Q}_{p}}\left(\operatorname{Sym}^{2} \mathrm{~V}_{p}\left(E_{2}\right)\right)(-1)\right)=0
$$

Proof. First, note that the assumptions in this corollary force $E_{1}$ to have root number -1 , that is, $\epsilon\left(\mathrm{h}\left(E_{1}\right)(1)\right)=-1$. In fact, we have

$$
\epsilon\left(\mathrm{h}\left(E_{1}\right)(1) \otimes \mathrm{h}\left(E_{2}\right)(1) \otimes \mathrm{h}\left(E_{2}\right)(1)\right)=\epsilon\left(\mathrm{h}\left(E_{1}\right)(1)\right) \times \epsilon\left(\mathrm{h}^{1}\left(E_{1}\right)(1) \otimes\left(\operatorname{Sym}^{2} \mathrm{~h}^{1}\left(E_{2}\right)\right)(1)\right),
$$

in which the first root number is -1 since $N_{1}$ and $N_{2}$ are coprime, and the last root number is 1 since $L\left(0, \mathrm{~h}^{1}\left(E_{1}\right)(1) \otimes\left(\operatorname{Sym}^{2} \mathrm{~h}^{1}\left(E_{2}\right)\right)(1)\right) \neq 0$.

Choose a prime $\ell \mid N_{1}$ such that $E_{1}$ has multiplicative reduction at $\ell$. In particular, we have $\ell \| N_{1}$. Choose a quadratic Dirichlet character $\eta_{1}$ of conductor coprime to $N_{1}$ such that $\eta_{1}(-1)=1, \eta_{1}(\ell)=-1$, and $\eta_{1}\left(\ell^{\prime}\right)=1$ for all other prime factors $\ell^{\prime}$ of $N_{1}$. Then the quadratically twisted elliptic curve $E_{1}^{\eta_{1}}$ has root number 1 . By the main theorem of [BFH90], we may choose another quadratic character $\eta_{2}$ of conductor coprime to $N_{1}$ such that $\eta_{2}(-1)=1, \eta_{2}\left(\ell^{\prime}\right)=1$ for all prime factors $\ell^{\prime}$ of $N_{1}$ (including $\ell$ ), and moreover $L\left(0, \mathrm{~h}\left(E_{1}^{\eta}\right)(1)\right) \neq 0$ where $\eta=\eta_{1} \eta_{2}$. Now let $F$ be the (real) quadratic field determined by $\eta$. Applying Theorem 1.3 with $E=E_{1}$ and $A=E_{2} \otimes_{\mathbb{Q}} F$, we have that for all but finitely many primes $p$,

$$
\operatorname{dim}_{\mathbb{Q}_{p}} \mathrm{H}_{f}^{1}\left(\mathbb{Q}, \mathrm{V}_{p}\left(E_{1}\right) \otimes_{\mathbb{Q}_{p}}\left(\operatorname{Sym}^{2} \mathrm{~V}_{p}\left(E_{2}\right)\right)(-1)\right)+\operatorname{dim}_{\mathbb{Q}_{p}} \mathrm{H}_{f}^{1}\left(\mathbb{Q}, \mathrm{V}_{p}\left(E_{1}^{\eta}\right)\right)=0,
$$

which implies the corollary.

1.4. Analogy with Kolyvagin's work. The current article is mostly influenced by the pioneer work of Kolyvagin. We explain this in the case where $(E, A)$ is of odd type.

Let $E$ be an elliptic curve over $\mathbb{Q}$ of conductor $N$ with the associated cuspidal automorphic representation $\sigma$ of $\mathrm{GL}_{2}(\mathbb{A})$. Let $K \subset \mathbb{Q}^{\text {ac }}$ be an imaginary quadratic field satisfying the (strong) Heegner condition for $E$ : every prime factor of $N$ is split 
in $K$. Denote by $Y_{N}$ the compactified modular curve over $\mathbb{Q}$ of $\Gamma_{0}(N)$ level structure. Pick up a Heegner point $P_{K}$ with respect to the maximal order of $K$ on $Y_{N}(\mathbb{C})$, which in fact belongs to $Y_{N}\left(H_{K}\right)$ where $H_{K}$ is the Hilbert class field of $K$. Take a modular parametrization $\phi: Y_{N} \rightarrow E$ that sends the cusp to $0_{E}$. Define

$$
P_{K, E}=\operatorname{Tr}_{H_{K} / K}\left(\phi\left(P_{K}\right)-0_{E}\right) \in \mathrm{CH}^{1}\left(E_{K}\right)^{0}=\mathrm{CH}\left(\mathrm{h}^{1}\left(E_{K}\right)(1)\right) \text {. }
$$

In [Kol90], Kolyvagin proved that if $P_{K, E} \neq 0$, then $\operatorname{dim}_{\mathbb{Q}_{p}} \mathrm{H}_{f}^{1}\left(K, \mathrm{~h}^{1}\left(E_{K^{\mathrm{ac}}}\right)(1)_{p}\right)=1$ for all primes $p$. By the Gross-Zagier formula [GZ86], we have that $P_{K, E} \neq 0$ if and only if $L^{\prime}\left(1 / 2, \sigma_{K}\right) \neq 0$. Note that $L\left(s, \mathrm{~h}^{1}\left(E_{K}\right)(1)\right)=L\left(s+1 / 2, \sigma_{K}\right)$, with root number $\epsilon\left(\mathrm{h}^{1}\left(E_{K}\right)(1)\right)=-1$.

Remark 1.21. Although we use the same notation for $E$, the reader should not think it is the same thing as in the pair $(E, A)$ previously. In fact, the role $E$ plays in Kolyvagin's case is more or less the role of the other elliptic curve $A$ in our case.

We summarize the analogy between Kolyvagin's setup and ours in Table 1.

\begin{tabular}{c||c|c}
\hline & Kolyvagin's case & our case \\
\hline motive & $\mathrm{h}^{1}\left(E_{K}\right)(1)$ & $\mathrm{M}_{E, A}$ \\
\hline Hodge-Tate weights & $\{-1,0\}$ & $\left\{-2,-1^{3}, 0^{3}, 1\right\}$ \\
\hline automorphic $L$-function & $L\left(s, \sigma_{K}\right)$ & $L(s, \sigma \times \pi)$ \\
\hline special cycle & $P_{K, E}$ & $\Delta_{E, A}$ \\
\hline GGP pair $H \times G$ & $\mathrm{SO}(2) \times \mathrm{SO}(3)$ & $\mathrm{SO}(3) \times \mathrm{SO}(4)$ \\
\hline ambient variety $Y \times X$ & CM 0-fold $\times$ modular curve & Shimura curve $\times \mathrm{HMS}$ \\
\hline \multicolumn{2}{|c}{ TABLE 1. Analogy }
\end{tabular}

In fact, the key idea of our proof is similar to Kolyvagin's as well. Namely, we use variants of $\Delta_{E, A}\left(P_{K, E}\right.$ in Kolyvagin's case) to produce sufficiently many annihilators, under the Tate pairing, of the Selmer group of the Galois cohomology with torsion coefficients. For $\mathrm{M}$ equal to $\mathrm{h}^{1}\left(E_{K}\right)(1)$ or $\mathrm{M}_{E, A}$, we have a natural Galois stable lattice $\overline{\mathrm{M}}_{p}^{\infty}$ of $\mathrm{M}_{p}$. For an integer $n \geq 1$, the "free rank" $r_{n}$ of the torsion Selmer group $\mathrm{H}_{f}^{1}\left(K, \overline{\mathrm{M}}_{p}^{n}\right)$, where $\overline{\mathrm{M}}_{p}^{n}=\overline{\mathrm{M}}_{p}^{\infty} \otimes_{\mathbb{Z}_{p}} \mathbb{Z} / p^{n}$, will be stabilized to $r_{\infty}$ as $n \rightarrow \infty$. Under both situations, the goal is to show that $r_{\infty}=1$.

In Kolyvagin's case, the cycle $P_{K, E}$ is constructed via the diagonal embedding $Y \rightarrow$ $Y \times X$ where $Y$ (resp. $X$ ) is the union of CM points (resp. modular curve), which is also the Shimura 0-fold (resp. 1-fold) attached to the group $H=\mathrm{SO}(2)$ defined by $K$ (resp. $\left.G=\mathrm{SO}(3)=\mathrm{PGL}_{2, \mathbb{Q}}\right)$. Here, the scheme $Y$ maps naturally to $X$ through an optimal embedding $H \hookrightarrow G$ of underlying groups. Besides $P_{K, E}$, he defined other classes $\bar{P}_{K, E \mid S}^{n}$ with torsion coefficient $\mathbb{Z} / p^{n}$ for $S$ a finite set of so-called Kolyvagin primes (with respect to $p^{n}$ ), by modifying the embedding $H \hookrightarrow G$ and hence $Y \rightarrow X$. These variant classes $\bar{P}_{K, E \mid S}^{n}$ are closely related to $P_{K, E}$. In fact, once $P_{K, E} \neq 0$, they will produce sufficiently many annihilators for the Selmer group $\mathrm{H}_{f}^{1}\left(K, \overline{\mathrm{M}}_{p}^{n}\right)$. In fact, it is enough to consider those classes with $\# S \leq 2$.

In our case, the cycle $\Delta_{E, A}$ is constructed via the diagonal embedding $Y \rightarrow Y \times X$ where $Y$ (resp. $X$ ) is a Shimura curve (resp. Hilbert modular surface), which is also 
the Shimura 1-fold (resp. 2-fold) attached to the group $H=\mathrm{SO}(3)$ defined by the quaternion algebra $B_{N^{-}}$(resp. $\left.G=\mathrm{SO}(4) \subset \operatorname{Res}_{F / \mathbb{Q}} \mathrm{GL}_{2, F} / \mathbf{G}_{m, \mathbb{Q}}\right)$. Besides $\Delta_{E, A}$, we also define other classes $\bar{\Delta}_{E, A \mid \ell_{1}, \ell_{2}}$ with torsion coefficient $\mathbb{Z} / p^{n}$ for $\ell_{1}, \ell_{2}$ being two distinct strongly $n$-admissible primes, as we call. For doing this, instead of changing the embedding $H \hookrightarrow G$ as in Kolyvagin's case, we change the algebra $B_{N^{-}}$and hence the group $H$. Such idea first appeared in the work of Bertolini-Darmon [BD05]. The most difficult part is to relate these variant classes $\bar{\Delta}_{E, A \mid \ell_{1}, \ell_{2}}^{n}$ to the original one in an explicit way. Once this is achieved and ${ }^{p} \Delta_{E, A} \neq 0$, they will produce sufficiently many annihilators for the Selmer group $\mathrm{H}_{f}^{1}\left(K, \overline{\mathrm{M}}_{p}^{n}\right)$, by some sophisticated Galois-theoretical arguments.

1.5. Strategy of proof. We explain in more details about our strategy of the proof, and take the chance to introduce the main structure of the article. The proofs for Theorem 1.3 and Theorem 1.5 will be carried out simultaneously. To avoid confusion, here we will restrict ourselves to the case where $(E, A)$ is of odd type. In fact, the ingredients we need for the proof of Theorem 1.5 strictly contain those of Theorem 1.3.

In $\S 2$, we introduce and study some properties of our fundamental geometric objects, namely, Shimura curves and the Hilbert modular surface. We give canonical parametrization of the following objects:

- supersingular locus of a Shimura curve at a good prime,

- reduction graph of a Shimura curve in the Čerednik-Drinfeld reduction,

- reduction graph of the supersingular locus of a Hilbert modular surface at a good inert prime.

Note that the first two are known by the work of Ribet [Rib89]. We will use a similar method to study the third one. Here, the canonical parametrization is realized by oriented orders in certain definite quaternion algebras, without picking up a base point. More importantly, we will study the behavior of canonical parametrization under special morphisms from different Shimura curves to the Hilbert modular surface (which is fixed in application) at different primes. The canonicality of the parametrization is crucial for doing this (see Propositions 2.24 and 2.25). All these considerations will be used in the computation of localization maps later.

We start $\S 3$ by recalling the definition of Bloch-Kato Selmer groups in various setting, and proving some preparatory results. Then we switch the language from elliptic curves to automorphic representations, followed by the construction of the cycle $\Delta_{E, A}$, or rather $\Delta_{\sigma, \pi}$ in the automorphic setting. After recalling certain results from [BD05] concerning level raising of torsion Galois modules for $\mathrm{GL}_{2, \mathbb{Q}}$, we introduce those classes $\bar{\Delta}_{\sigma, \pi \mid \ell_{1}, \ell_{2}}^{n}$. The most technical part is to choose appropriate primes, which we call strongly admissible primes, in the sense that there should be enough of them for those classes to serve as annihilators, and meanwhile they should be special enough to make the computation of localization possible.

The next chapter $\S 4$ is the technical core of the article, where we formulate and prove the explicit congruence formulae in Theorems 4.10, for $\Delta_{\sigma, \pi}$ and various $\bar{\Delta}_{\sigma, \pi \mid \ell_{1}, \ell_{2}}^{n}$ via localization. The computation relies on the existence of integral Tate cycles on special fibers of the Hilbert modular surface at good inert primes, together with certain 
specialization arguments from algebraic geometry. In fact, the localization of both $\Delta_{\sigma, \pi}$ and $\bar{\Delta}_{\sigma, \pi \mid \ell_{1}, \ell_{2}}^{n}$ can be realized as period integrals of Gan-Gross-Prasad type, of some torsion algebraic automorphic forms for certain pair $\mathrm{SO}(3) \times \mathrm{SO}(4)$ that are compact at infinity.

We arrive at the final stage of the proof in $\$ 5$. The remaining argument is a combination of Theorem 4.10, Tate pairings, Serre's theorems on large Galois image for elliptic curves, and some Galois-theoretical techniques, in particular, the heavy use of the Chebotarev Density Theorem. The final part of the proof is much more involved than Kolyvagin's case, essentially due to the absence of Kummer maps and the inapplicability of Mordell-Weil group (Chow group).

\subsection{Notation and conventions.}

\subsubsection{Generalities.}

- The Greek letter $\epsilon$ always represents a sign, either + or - .

- All rings and algebras are unital and homomorphisms preserve units. For a ring, it is always assumed to be commutative.

- Throughout the article, a prime means a rational prime number; a prime power means a positive integer power of a prime. Starting from the next chapter, the prime $p$ is always odd.

- For an integer $N$, denote by $\wp(N) \geq 0$ the number of its distinct prime factors.

- For a ring $R$ of prime characteristic, denote by $\mathrm{W}(R)$ the ring of infinite Witt vectors with values in $R$.

- For every prime $\ell$, we fix an algebraic closure $\mathbb{F}_{\ell}^{\text {ac }}$ of $\mathbb{F}_{\ell}$. If $\ell^{f}$ is a prime power, then we denote by $\mathbb{F}_{\ell^{f}}$ the unique subfield of $\mathbb{F}_{\ell}^{\text {ac }}$ of cardinality $\ell^{f}$. If $v$ is a prime power, then we put $\mathbb{Z}_{v}=\mathrm{W}\left(\mathbb{F}_{v}\right)$ and $\mathbb{Q}_{v}=\mathbb{Z}_{v} \otimes_{\mathbb{Z}} \mathbb{Q}$.

- Denote by $\mathbb{A}$ (resp. $\mathbb{A}^{\infty}$ ) the ring of adèles (resp. finite adèles) of $\mathbb{Q}$.

- If $S$ is a set and $R$ is a ring, then we denote by $R[S]$ the $R$-module of functions from $S$ to $R$ with finite support. We have a degree map deg: $R[S] \rightarrow R$ sending $f$ to $\sum_{s \in S} f(s)$, and denote by $R[S]^{0}$ the kernel of the degree map.

\subsubsection{Algebraic geometry.}

- Denote by $\mathbf{P}^{1}$ the projective line scheme over $\mathbb{Z}$, and $\mathbf{G}_{m}=\operatorname{Spec} \mathbb{Z}\left[T, T^{-1}\right]$ the multiplicative group scheme.

- If $S$ is a scheme and $A$ is an $S$-abelian scheme, then we denote by $A^{\vee}$ its dual $S$-abelian scheme.

- If $X / S$ is a relative scheme and $S^{\prime}$ is an $S$-scheme, then we denote by $X_{S^{\prime}}=$ $X \times_{S} S^{\prime}$ for the base change. If $S^{\prime}=\operatorname{Spec} R$ is affine, then we write $X_{R}$ instead of $X_{S^{\prime}}$. If the notation for a scheme has already carried a subscript such as $X_{N^{+} M}$, then we denote by $X_{N^{+} M ; S^{\prime}}$ or $X_{N^{+} M ; R}$ for the base change.

- Let $S$ be a scheme, and $j: Y \rightarrow X$ be a closed immersion of $S$-schemes. For a sheaf $\mathscr{F}$ in the étale topos $X_{\text {ét }}$, denote by

$$
\mathrm{H}_{Y}^{i}\left(X_{S^{\prime}}, \mathscr{F}\right)=\mathrm{h}^{i} \mathrm{R} \Gamma\left(\left(Y_{S^{\prime}}\right)_{\text {ét }}, b^{*} j^{!} \mathscr{F}\right)
$$


the cohomology with support for an $S$-scheme $S^{\prime}$ and the base change morphism $b: Y_{S^{\prime}} \rightarrow Y$. If $S^{\prime}$ is a (pro-)Galois cover of $S$, then $\mathrm{H}_{Y}^{i}\left(X_{S^{\prime}} ; F\right)$ is equipped with a (continuous) action of the (pro-)finite Galois group $\operatorname{Gal}\left(S^{\prime} / S\right)$.

- Let $X$ be a smooth proper scheme over a field $k$ of characteristic zero. For each integer $r \geq 0$, denote by $\mathrm{CH}^{r}(X)^{0}$ the subspace of $\mathrm{CH}^{r}(X) \otimes_{\mathbb{Z}} \mathbb{Q}$ of cohomologically trivial cycles, that is, the kernel of the geometric cycle class map

$$
\mathrm{cl}_{X}^{0}: \mathrm{CH}^{r}(X) \otimes_{\mathbb{Z}} \mathbb{Q} \rightarrow \mathrm{H}_{\text {ét }}^{2 r}\left(X_{k^{\text {ac }}}, \mathbb{Q}_{p}(r)\right)
$$

for some and hence all primes $p$.

- Let $X$ be a smooth proper scheme over a field $k$ purely of dimension $d$. Denote by $\operatorname{Corr}(X)=\mathrm{CH}^{d}\left(X \times_{\text {Spec } k} X\right) \otimes_{\mathbb{Z}} \mathbb{Q}$ the $\mathbb{Q}$-algebra of correspondences (of degree 0 ) of $X$, with multiplication given by composition of correspondences. It acts on $\mathrm{CH}^{*}(X) \otimes_{\mathbb{Z}} \mathbb{Q}$ via pullbacks. If the characteristic of $k$ is 0 , then we denote by

$$
\mathrm{H}_{\mathrm{dR}}^{*}(X)=\bigoplus_{r=0}^{2 d} \mathrm{H}_{\mathrm{dR}}^{i}(X)
$$

the total de Rham cohomology of $X$, which is a graded $k$-vector space. We have a natural homomorphism

$$
\operatorname{cor}_{\mathrm{dR}}: \operatorname{Corr}(X) \rightarrow \operatorname{End}_{k}^{\mathrm{gr}}\left(\mathrm{H}_{\mathrm{dR}}^{*}(X)\right)
$$

of $\mathbb{Q}$-algebras via pullbacks.

\subsubsection{Galois modules.}

- For a field $k$, denote by $\Gamma_{k}=\operatorname{Gal}\left(k^{\mathrm{ac}} / k\right)$ the absolute Galois group of $k$. If $k=K$ is a subfield of $\mathbb{Q}^{\text {ac }}$, then we take $K^{\text {ac }}=\mathbb{Q}^{\text {ac }}$ and hence $\Gamma_{K} \subset \Gamma_{\mathbb{Q}}$.

- For an abelian variety $A$ over a field $k$ of characteristic not $p$, we put $\mathrm{T}_{p}(A)=$ $\lim _{m} A\left[p^{m}\right]\left(k^{\mathrm{ac}}\right)$ which is a $\mathbb{Z}_{p}$-module with a continuous $\Gamma_{k}$-action.

- We simply write $\mathrm{H}^{\bullet}(k,-)$ instead of $\mathrm{H}^{\bullet}\left(\Gamma_{k},-\right)$ for group cohomology of the absolute Galois group $\Gamma_{k}$.

- If a group $G$ acts on a set $V$, then $V^{G}$ is the subset of $G$-fixed elements. We write $V^{k}$ instead of $V^{\Gamma_{k}}$.

- If $k$ is a local field, then we denote by $\mathrm{I}_{k} \subset \Gamma_{k}$ the inertia subgroup, and $\mathrm{Fr}_{k}$ the arithmetic Frobenius element of $\Gamma_{k} / \mathrm{I}_{k}$. If $v$ is a prime power, then we simply write $\Gamma_{v}$ for $\Gamma_{\mathbb{Q}_{v}}, \mathrm{I}_{v}$ for $\mathrm{I}_{\mathbb{Q}_{v}}$, and $\mathrm{Fr}_{v}$ for $\operatorname{Fr}_{\mathbb{Q}_{v}}$.

- Let $R$ be a ring and $\mathrm{T}$ be an $R\left[\Gamma_{\mathbb{Q}}\right]$-module. For each prime power $v$, we have the following localization map

$$
\operatorname{loc}_{v}: \mathrm{H}^{1}(\mathbb{Q}, \mathrm{T}) \rightarrow \mathrm{H}^{1}\left(\mathbb{Q}_{v}, \mathrm{~T}\right)
$$

of $R$-modules obtained by restriction. For $r \in R^{\times}$, we denote by $\mathrm{T}[v \mid r]$ the largest $R$-submodule of $\mathrm{T}$ on which $\mathrm{I}_{v}$ acts trivially and $\mathrm{Fr}_{v}$ acts by multiplication by $r$.

- If $v$ is a prime power and $\mathrm{T}$ is an $R\left[\Gamma_{v}\right]$-module, then we put

$$
\mathrm{H}_{\mathrm{unr}}^{1}\left(\mathbb{Q}_{v}, \mathrm{~T}\right)=\operatorname{Ker}\left[\mathrm{H}^{1}\left(\mathbb{Q}_{v}, \mathrm{~T}\right) \rightarrow \mathrm{H}^{1}\left(\mathrm{I}_{v}, \mathrm{~T}\right)\right]
$$


as an $R$-submodule of $\mathrm{H}^{1}\left(\mathbb{Q}_{v}, \mathrm{~T}\right)$. Put $\mathrm{H}_{\text {sing }}^{1}\left(\mathbb{Q}_{v}, \mathrm{~T}\right)=\mathrm{H}^{1}\left(\mathbb{Q}_{v}, \mathrm{~T}\right) / \mathrm{H}_{\text {unr }}^{1}\left(\mathbb{Q}_{v}, \mathrm{~T}\right)$ with the quotient map $\partial_{v}: \mathrm{H}^{1}\left(\mathbb{Q}_{v}, \mathrm{~T}\right) \rightarrow \mathrm{H}_{\text {sing }}^{1}\left(\mathbb{Q}_{v}, \mathrm{~T}\right)$.

- Let $\mathrm{G}$ be a topological group. By a p-adic representation, we mean a pair $(\rho, \mathrm{V})$ where $\mathrm{V}$ is a finite dimensional $\mathbb{Q}_{p}$-vector space, and $\rho: \mathrm{G} \rightarrow \mathrm{GL}(\mathrm{V})$ is a continuous homomorphism. A stable lattice in $(\rho, \mathrm{V})$ is a finitely generated $\mathbb{Z}_{p}$-submodule $\mathrm{T} \subset \mathrm{V}$ such that $\mathrm{T} \otimes_{\mathbb{Z}_{p}} \mathbb{Q}_{p}=\mathrm{V}$ and $\mathrm{T}$ is preserved under the action of $\mathrm{G}$. Sometimes we will omit $\rho$ if it is irrelevant.

- Let $\mathrm{T}$ be a stable lattice in a $p$-adic representation $(\rho, \mathrm{V})$ of $\mathrm{G}$. For $n \geq 1$, we denote by

$$
\bar{\rho}^{n}: \mathrm{G} \rightarrow \mathrm{GL}\left(\overline{\mathrm{T}}^{n}\right)
$$

the induced residue representation, where $\overline{\mathrm{T}}^{n}=\mathrm{T} \otimes_{\mathbb{Z}_{p}} \mathbb{Z} / p^{n}$. Usually we omit the superscript $n$ when it is 1 . In certain cases, we also allow $n=\infty$, then $\overline{\mathrm{T}}^{\infty}$ is nothing but $\mathrm{T}$, and $\mathbb{Z} / p^{\infty}$ is understood as $\mathbb{Z}_{p}$.

Acknowledgements. The author would like to thank Henri Darmon, Wee-Tech Gan, Benedict Gross, Atsushi Ichino, Ye Tian, Yichao Tian, Shouwu Zhang, and Wei Zhang for helpful discussions and comments. He appreciates Yichao Tian and Liang Xiao for sharing their preprint [TX14] at the early stage. He also thank the anonymous referees for very careful reading and useful comments. The author is partially supported by NSF grant DMS-1302000.

\section{Geometry of some modular schemes}

In this chapter, we study geometry of some modular schemes which we will use. In $\S 2.1$, we collect some notation and facts about certain quaternion algebras and their orders. In $\S 2.2$, we study the canonical parametrization of supersingular abelian surfaces using quaternion orders. In $\S 2.3$, we study geometry of Shimura curves, focusing on the special fibers of both smooth and semistable reductions. In $\$ 2.4$, we study geometry of Hilbert modular surfaces and their reduction at good inert primes. In $\$ 2.5$, we consider the connection between Shimura curves and Hilbert modular surfaces through Hirzebruch-Zagier morphisms, particularly the interaction of their reductions under such morphisms.

2.1. Algebraic preparation. We first introduce a bunch of notation.

Notation 2.1. Recall that $F$ is a real quadratic number field.

(1) Denote by $\mathrm{Cl}(F)^{+}$the strict ideal class group of $F$, whose elements are represented by projective $O_{F}$-modules of rank 1 with a notion of positivity. Denote by $\mathfrak{D}$ the different of $F$. Then for example, the pair $\left(\mathfrak{D}^{-1},\left(\mathfrak{D}^{-1}\right)^{+}\right)$is an element in $\mathrm{Cl}(F)^{+}$of order at most 2. See [Gor02, Chapter 2, §1] for more details.

(2) For each prime $\ell$ that is inert in $F$, we fix an embedding $\tau_{\ell}^{\bullet}: O_{F, \ell}:=O_{F} \otimes_{\mathbb{Z}} \mathbb{Z}_{\ell} \hookrightarrow$ $\mathrm{W}\left(\mathbb{F}_{\ell}^{\mathrm{ac}}\right)$ of $\mathbb{Z}_{\ell}$-algebras, and put $\tau_{\ell}^{\circ}=\tau_{\ell}^{\bullet} \circ \theta$. They induce (by the same notation) two homomorphisms $\tau_{\ell}^{\bullet}, \tau_{\ell}^{\circ}: O_{F, \ell} \rightarrow O_{F} / \ell \hookrightarrow \mathbb{F}_{\ell}^{\text {ac }}$.

Notation 2.2. Let $D$ be a square-free positive integer. 
(1) We denote by $B_{D}$ the unique indefinite (resp. definite) quaternion algebra over $\mathbb{Q}$ which ramifies exactly at primes dividing $D$ when $D$ has even (resp. odd) number of prime factors. Denote by $B_{D}^{+} \subset B_{D}$ the subset of elements with positive reduced norm.

(2) Suppose that $D$ has even number of prime factors. Denote by $\iota$ the canonical involution on $B_{D}$. Let $\mathcal{O}_{D}$ be a maximal order of $B_{D}$. Fix $\delta \in \mathcal{O}_{D}$ with $\delta^{2}=-D$. Define another involution $*$ on $B_{D}$ by the formula $b^{*}=\delta^{-1} b^{\iota} \delta$. It is easy to see that $\mathcal{O}_{D}$ is preserved under $*$.

(3) Suppose that $D$ has even number of prime factors, all being inert in $F$. We will fix an oriented maximal order $\left(\mathcal{O}_{D}, o_{v}\right)$ in $B_{D}$, which by Strong Approximation [Vig80, Chaptire III, Théorème 4.3] is unique up to conjugation in $B_{D}$, together with an embedding $O_{F} \hookrightarrow \mathcal{O}_{D}$ of $\mathbb{Z}$-algebras. Recall that an orientation of $\mathcal{O}_{D}$ is a homomorphism $o_{v}: \mathcal{O}_{D} \rightarrow \mathbb{F}_{v^{2}}$ for each prime $v \mid D$. We require that the restriction $\left.o_{v}\right|_{O_{F}}$ coincides with $\tau_{v}^{\bullet}$. Put $\mathfrak{M}_{D}=\left\{b \in \mathcal{O}_{D} \mid x b=b^{*} x^{*}, \forall x \in O_{F}\right\}$ and $\mathfrak{M}_{D}^{+}=\mathfrak{M}_{D} \cap B_{D}^{+}$. Then $\left(\mathfrak{M}_{D}, \mathfrak{M}_{D}^{+}\right) \simeq\left(\mathfrak{D}^{-1},\left(\mathfrak{D}^{-1}\right)^{+}\right)$. We fix such an isomorphism $\psi_{D}$ once and for all.

(4) Suppose that $D$ has odd number of prime factors. If $M$ is another positive integer coprime to $D$, then we denote by $\mathcal{T}_{M, D}$ the set of isomorphism classes of oriented Eichler orders of level $M$ in $B_{D}$.

(5) For positive integers $d^{\prime}|d| M$, we have a degeneracy map

$$
\delta_{M, D}^{\left(d, d^{\prime}\right)}: \mathcal{T}_{M, D} \rightarrow \mathcal{T}_{M / d, D}
$$

Recall that an oriented Eichler order of level $M$ in $B_{D}$ (see [Rib89, §2]) is a tuple $\vec{R}=\left\{R, R^{\prime}, o_{v}\right\}$ where

- $R$ is an Eichler order of level $M$ in $B_{D}$;

- $R^{\prime}$ is a maximal Eichler order such that $R=R^{\prime} \cap R^{\prime \prime}$ for another (unique) maximal Eichler order $R^{\prime \prime}$;

- $o_{v}: R \rightarrow \mathbb{F}_{v^{2}}$ is a homomorphism of $\mathbb{Z}$-algebras for every prime $v \mid D$.

For the degeneracy map, suppose that $\vec{R}=\left(R, R^{\prime}, o_{v}\right)$ is an oriented Eichler order of level $M$ in $B_{D}$. Let $R_{1}$ be the unique Eichler order of level $M / d$ containing $R$ such that $\#\left(R^{\prime} / R^{\prime} \cap R_{1}\right)=M d^{\prime} / d$; and $R_{1}^{\prime}$ be the unique maximal Eichler order containing $R_{1}$ such that $\#\left(R^{\prime} / R^{\prime} \cap R_{1}^{\prime}\right)=d^{\prime}$. Then we define $\delta_{M, D}^{\left(d, d^{\prime}\right)}(\vec{R})$ to be $\left(R_{1}, R_{1}^{\prime}, o_{v}\right)$.

For each prime $\ell$ such that $\ell^{s} \| M$ with $s \geq 1$, we have a unique involution switching the orientation at $\ell$

$$
\mathrm{op}_{\ell}: \mathcal{T}_{M, D} \rightarrow \mathcal{T}_{M, D},
$$

such that $\delta_{M, D}^{\left(\ell^{s}, \ell^{s}\right)}=\delta_{M, D}^{\left(\ell^{s}, 1\right)} \circ \mathrm{op}_{\ell}$. If $\ell \mid D$, then we also have an involution switching the orientation at $\ell$

$$
\mathrm{op}_{\ell}: \mathcal{T}_{M, D} \rightarrow \mathcal{T}_{M, D}
$$

sending $\vec{R}=\left(R, R^{\prime}, o_{v}\right)$ to the one obtained by only switching $o_{\ell}$ to its conjugate.

Notation 2.3. Let $\mathfrak{M}$ be an ideal of $O_{F}$. 
(1) Denote by $Q$ the unique totally definite quaternion algebra over $F$ that is unramified at all finite places. Denote by $\mathcal{S}_{\mathfrak{M}}$ the set of isomorphism classes of oriented $\left(O_{F}\right.$-linear) Eichler orders $\vec{R}=\left(R, R^{\prime}\right)$ of level $\mathfrak{M}$ in $Q$.

(2) For ideals $\mathfrak{d}^{\prime}|\mathfrak{d}| \mathfrak{M}$, we have a similar degeneracy map

$$
\gamma_{\mathfrak{M}}^{\left(\mathfrak{d}, \mathfrak{o}^{\prime}\right)}: \mathcal{S}_{\mathfrak{M}} \rightarrow \mathcal{S}_{\mathfrak{M} / \mathfrak{d}}
$$

(3) For each prime ideal $\mathfrak{l}$ of $O_{F}$ such that $\mathfrak{l} \mid \mathfrak{M}$, we have a similar involution switching the orientation at $\mathfrak{l}$

$$
\mathrm{op}_{\mathfrak{l}}: \mathcal{S}_{\mathfrak{M}} \rightarrow \mathcal{S}_{\mathfrak{M}}
$$

Definition 2.4. Suppose that $D$ has even number of prime factors, all being inert in $F$. We would like to construct a canonical special map

$$
\zeta_{M, D}: \mathcal{T}_{M, D} \rightarrow \mathcal{S}_{M}
$$

as follows. Take $\vec{R}=\left(R, R^{\prime}, o_{v}\right) \in \mathcal{T}_{M, D}$. There is a unique $\left(O_{F}\right.$-linear $)$ Eichler order $R_{\sharp}$ of level $M$ in the $F$-quaternion algebra $B_{D} \otimes_{\mathbb{Q}} F \simeq Q$ such that it contains $R \otimes_{\mathbb{Z}} O_{F}$, and for every prime $v \mid D$, the $\left(R / v, O_{F} / v\right)$-bimodule $R_{\sharp} \otimes_{\mathbb{Z}} \mathbb{Z}_{v} / R \otimes_{\mathbb{Z}} O_{F, v} \simeq \mathbb{F}_{v^{2}}$ is isomorphic to $o_{v} \otimes \tau_{v}^{\bullet}$. Similarly, one defines $R_{\sharp}^{\prime}$. Then we define $\zeta_{M, D}(\vec{R})$ to be $\left(R_{\sharp}, R_{\sharp}^{\prime}\right)$. The special maps $\zeta_{M, D}$ are compatible with degeneracy maps in the sense that the following diagram

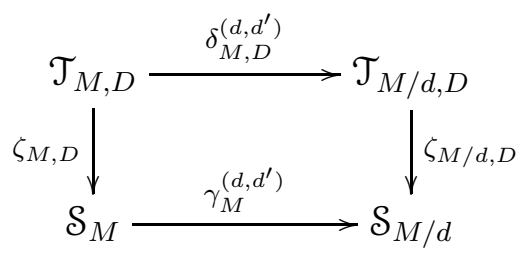

commutes for all $d^{\prime}|d| M$.

Let $\ell$ be a prime that is inert in $F$. Fix an oriented maximal order $\left(\mathcal{O}_{\ell}, o_{\ell}\right)$ of $B_{\ell}$. For each prime $v$, put $\mathcal{O}_{\ell, v}=\mathcal{O}_{\ell} \otimes_{\mathbb{Z}} \mathbb{Z}_{v}$. For $?=\bullet$, o, fix an embedding $\varsigma_{\ell}^{?}: O_{F, \ell} \hookrightarrow \mathcal{O}_{\ell, \ell}$ of $\mathbb{Z}_{\ell}$-algebras such that its composition with $o_{\ell}$ coincides with $\tau_{\ell}^{?}$.

Lemma 2.5. Let $n$ be a positive integer. Let $f: O_{F, \ell} \rightarrow \operatorname{Mat}_{n}\left(\mathcal{O}_{\ell, \ell}\right)$ be a homomorphism of $\mathbb{Z}_{\ell}$-algebras.

(1) Then $f$ is $\mathrm{GL}_{n}\left(\mathcal{O}_{\ell, \ell}\right)$-conjugate to a homomorphism of the form

$$
x \mapsto \operatorname{diag}\left[\varsigma_{\ell}^{\bullet}(x), \cdots, \varsigma_{\ell}^{\bullet}(x), \varsigma_{\ell}^{\circ}(x), \cdots, \varsigma_{\ell}^{\circ}(x)\right] .
$$

We say that $f$ is of type $(r, n-r)$ if $\varsigma_{\ell}^{\bullet}$ appears with $r$ times.

(2) If $f$ is of type $(r, n-r)$, then the commutator of $f\left(O_{F, \ell}\right)$ in $\operatorname{Mat}_{n}\left(\mathcal{O}_{\ell, \ell}\right)$ is isomorphic to $\operatorname{End}\left(O_{F, \ell}^{\oplus r} \oplus \ell O_{F, \ell}^{\oplus n-r}\right) \cap \operatorname{Mat}_{n}\left(O_{F, \ell}\right)$ as an $O_{F, \ell}$-algebra.

Proof. The homomorphism $f$ corresponds to an $O_{F, \ell} \otimes_{\mathbb{Z}_{\ell}} \mathcal{O}_{\ell, \ell}$-module $M_{f}$, free of finite rank over $\mathcal{O}_{\ell, \ell}$. Since $O_{F, \ell} \otimes_{\mathbb{Z}_{\ell}} \mathcal{O}_{\ell, \ell}$ is an Eichler order of level $\ell$ in $\operatorname{Mat}_{2}\left(F_{\ell}\right)$, the $O_{F, \ell} \otimes_{\mathbb{Z}_{\ell}} \mathcal{O}_{\ell, \ell}$-module $M_{f}$ is isomorphic to a direct sum of $\mathcal{O}_{\ell, \ell}$ on which $\mathcal{O}_{\ell, \ell}$ acts by right multiplication, and $O_{F, \ell}$ acts from left via either $s_{\ell}^{\bullet}$ or $s_{\ell}^{\circ}$. This proves (1), of which (2) is a corollary. 
Now we study $\left(O_{F}, \mathcal{O}_{\ell}\right)$-bimodules of $(\mathbb{Z}$ - $)$ rank 8 . For such a bimodule $M$, consider the $\left(O_{F, \ell}, \mathcal{O}_{\ell, \ell}\right)$-bimodule $M_{\ell}:=M \otimes_{\mathbb{Z}} \mathbb{Z}_{\ell}$ which corresponds to a homomorphism $f_{M}: O_{F, \ell} \rightarrow \operatorname{Mat}_{2}\left(\mathcal{O}_{\ell, \ell}\right)$. We say that $M$ is pure (resp. mixed) if $f_{M}$ is of type $(0,2)$ or $(2,0)$ (resp. $(1,1))$. We deduce the following facts from the above lemma: If $M$ is pure, then $\operatorname{End}_{\left(O_{F}, \mathcal{O}_{\ell}\right)}(M)$, as an $O_{F}$-module, is isomorphic to a maximal order in $Q$, that is, an element in $\mathcal{S}_{1}$. If $M$ is mixed, then there is a unique sub-bimodule $M_{\bullet}$ of index $\ell^{2}$ that is of type $(0,2)$, and the inclusion $\operatorname{End}_{\left(O_{F}, \mathcal{O}_{\ell}\right)}(M) \subset \operatorname{End}_{\left(O_{F}, \mathcal{O}_{\ell}\right)}\left(M_{\bullet}\right)$ is naturally an element in $\mathcal{S}_{\ell}$. In all cases, we simply denote by $\operatorname{End}(M)$ the endomorphism $O_{F}$-algebra with the above orientation.

Proposition 2.6. The assignment $M \mapsto \operatorname{End}(M)$ induces a bijection between the set of isomorphism classes of pure of a fixed type (resp. mixed) $\left(O_{F}, \mathcal{O}_{\ell}\right)$-bimodules of rank 8 , and $\mathcal{S}_{1}\left(\right.$ resp. $\left.\mathcal{S}_{\ell}\right)$.

This proposition is the analogue of [Rib89, Theorem 2.4] for $\left(O_{F}, \mathcal{O}_{\ell}\right)$-bimodules. Note that in our case, the notion of admissibility is unnecessary. The proof of this proposition is very similar to that of [Rib89, Theorem 2.4]. Therefore, we only indicate necessary changes in the following proof, instead of explicating all the details.

Proof. We first prove that the assignment $M \mapsto \operatorname{End}(M)$ is injective. Choose an $\left(O_{F}, \mathcal{O}_{\ell}\right)$-bimodule $M$ as a reference point, and put $\Lambda=\operatorname{End}_{\left(O_{F}, \mathcal{O}_{\ell}\right)}(M)$. For each $\left(O_{F}, \mathcal{O}_{\ell}\right)$-bimodule $N$ that is locally isomorphic to $M$, put $J(N)=\operatorname{Hom}(M, N)$ as a right $\Lambda$-module which is locally free of rank 1 . The same proof for [Rib89, Theorem 2.3] implies that the assignment $N \mapsto J(N)$ establishes a bijection between the sets of isomorphism classes of

- $\left(O_{F}, \mathcal{O}_{\ell}\right)$-bimodules that are locally isomorphic to $M$;

- locally free right $\Lambda$-modules of rank 1 .

Therefore, the injectivity amounts to say that for $M$ and $M^{\prime}$ with isomorphic oriented Eichler order $\operatorname{End}(M) \simeq \operatorname{End}\left(M^{\prime}\right)$, the right $\Lambda$-module $\operatorname{Hom}\left(M, M^{\prime}\right)$ is free of rank 1 . The rest of the argument is the same as the one for [Rib89, Theorem 2.4], which works for an arbitrary Dedekind ring, in particular $O_{F}$, instead of $\mathbb{Z}$.

We then prove that the assignment $M \mapsto \operatorname{End}(M)$ is surjective. The first step is to show that there exists a mixed $\left(O_{F}, \mathcal{O}_{\ell}\right)$-bimodule of rank 8 , which has been constructed in the proof of [Rib89, Theorem 2.4]. The rest of the proof is a counting argument, which apparently works for $Q$ as well.

2.2. Some preparations on abelian surfaces. Fix an odd prime $\ell$ that is inert in $F$, and an ideal $\mathfrak{M}$ of $O_{F}$ coprime to $\ell$. Let $k$ be an algebraically closed field containing $\mathbb{F}_{\ell^{2}}$. Consider triples $(A, \iota, C)$ where $A$ is a $k$-abelian surface, $\iota: O_{F} \rightarrow \operatorname{End}(A)$ is a homomorphism of $\mathbb{Z}$-algebras, and $C$ is a level-M structure, that is, a subgroup of $A(k)$ of order $\mathrm{Nm}_{F / \mathbb{Q}} \mathfrak{M}$ that is stable and cyclic under the action of $O_{F}$.

Definition 2.7. We say that the triple $(A, \iota, C)$ is

(1) •-pure if $A$ is superspecial and $O_{F} / \ell$ acts on the $k$-vector space Lie $A$ by $\tau_{\ell}^{\bullet}$;

(2) o-pure if $A$ is superspecial and $O_{F} / \ell$ acts on the $k$-vector space Lie $A$ by $\tau_{\ell}^{\circ}$;

(3) mixed if both $\tau_{\ell}^{\bullet}$ and $\tau_{\ell}^{\circ}$ appear in the action of $O_{F} / \ell$ on Lie $A$; 
(4) superspecial mixed if it is mixed and $A$ is superspecial.

It is clear that the Frobenius element $\mathrm{Fr}_{\ell}$ acts on the set of all isomorphism classes of triples by sending $(A, \iota, C)$ to its Frobenius twist $\left(A^{(\ell)}, \iota^{(\ell)}, C^{(\ell)}\right)$.

For each triple $(A, \iota, C)$, denote by $A[\ell]_{\alpha}$ the maximal $\alpha_{\ell}$-elementary subgroup of $A[\ell]$, which is isomorphic to $\alpha_{\ell}^{\oplus a(A)}$ for some number $a(A) \in\{0,1,2\}$. Then $A$ is superspecial if and only if $a(A)=2$. For each mixed triple $(A, \iota, C)$ with $a(A)=1$, we say that it is - -mixed (resp. o-mixed) if $O_{F} / \ell$ acts on the Dieudonné module of $A[\ell]_{\alpha}$ via $\tau_{\ell}^{\bullet}\left(\right.$ resp. $\left.\tau_{\ell}^{\circ}\right)$. Given a $\bullet$-pure (resp. o-pure) triple $(A, \iota, C)$ and a subgroup $H \subset A[\ell]_{\alpha}$ that is isomorphic to $\alpha_{\ell}$ (in particular, $H$ is automatically stable under the action of $\left.\iota\left(O_{F}\right)\right)$, we have an induced triple $(A / H, \iota, C)$. It is either $\bullet$-mixed (resp. o-mixed) or superspecial mixed. The set of such subgroups $H$ is isomorphic to $\mathbf{P}^{1}(k)$. In fact, one can construct a family, called Moret-Bailly family (see, for example, [Nic00, §4]), of triples over $\mathbf{P}_{k}^{1}$ parameterizing $\{(A / H, \iota, C) \mid H\}$. There are exactly $\ell^{2}+1=\# \mathbf{P}^{1}\left(\mathbb{F}_{\ell^{2}}\right)$ subgroups $H$ such that $(A / H, \iota, C)$ is superspecial mixed.

Remark 2.8. If we start with a superspecial mixed triple $(A, \iota, C)$, then there is a unique $O_{F}$-stable subgroup $H_{\bullet}\left(\right.$ resp. $\left.H_{\circ}\right)$ of $A[\ell]_{\alpha}$ isomorphic to $\alpha_{\ell}$ such that $O_{F} / \ell$ acts on its Dieudonné module by $\tau_{\ell}^{\bullet}\left(\right.$ resp. $\left.\tau_{\ell}^{\circ}\right)$. Dividing it, we obtain a triple $\left(A / H_{\bullet}, \iota, C\right)$ (resp. $\left.\left(A / H_{\circ}, \iota, C\right)\right)$ that is o-pure (resp. - -pure). Moreover, the triple $\left(A^{(\ell)}, \iota^{(\ell)}, C^{(\ell)}\right)$ appears in the Moret-Bailly family associated to $\left(A / H_{\bullet}, \iota, C\right)$ (resp. $\left.\left(A / H_{\circ}, \iota, C\right)\right)$, as obtained by dividing $A[\ell]_{\alpha} / H_{\bullet}$ (resp. $\left.A[\ell]_{\alpha} / H_{\circ}\right)$.

For each $(A, \iota, C)$, denote by $\operatorname{End}(A, \iota, C)$ the $O_{F}$-algebra of endomorphisms of $A$ that commute with $\iota\left(O_{F}\right)$ and preserve $C$. If $(A, \iota, C)$ is pure (resp. superspecial mixed), then $\operatorname{End}(A, \iota, C)$ is an Eichler order of level $\mathfrak{M}$ (resp. $\mathfrak{M} \ell)$ in $Q$. Moreover, we endow $\operatorname{End}(A, \iota, C)$ with the orientation given by the maximal order $\operatorname{End}(A, \iota)$ (resp. $\left.\operatorname{End}\left(A / H_{\bullet}, \iota\right)\right)$.

Proposition 2.9. The assignment $(A, \iota, C) \mapsto \operatorname{End}(A, \iota, C)$, with the above orientation, induces a bijection between

(1) the set of isomorphism classes of $\bullet$-pure triples and $\mathcal{S}_{\mathfrak{M}}$;

(2) the set of isomorphism classes of o-pure triples and $\mathcal{S}_{\mathfrak{M}}$;

(3) the set of isomorphism classes of superspecial mixed triples and $\mathcal{S}_{\mathfrak{M} \ell}$.

Moreover, the Frobenius element $\mathrm{Fr}_{\ell}$

- switches the types of •-pure and o-pure and does not change values in $\mathcal{S}_{\mathfrak{M}}$;

- preserves the set of superspecial mixed triples and coincides with $\mathrm{op}_{\ell}$ on $\mathcal{S}_{\mathfrak{M} \ell}$.

Proof. The proof of this proposition follows exactly as for [Rib89, Theorems $4.15 \&$ 4.16], in which we only need to replace [Rib89, Theorem 2.14] by Proposition 2.6. The action of $\mathrm{Fr}_{\ell}$ follows from the construction.

2.3. Shimura curves. Let $N^{-}$be a square-free positive integer such that $\wp\left(N^{-}\right)$is even, and $N^{+}$be another integer coprime to $N^{-}$. Denote by $y_{N^{+}, N^{-}}$the functor from the category of schemes over $\mathbb{Z}\left[1 / N^{+}\right]$to the category of groupoids such that $y_{N^{+}, N^{-}}(S)$ is the groupoid of triples $(A, \iota, C)$, where 
- $A$ is an $S$-abelian scheme of relative dimension 2 ;

- $\iota: \mathcal{O}_{N^{-}} \rightarrow \operatorname{End}(A)$ is a special action (see [BC91, §III.3]) of $\mathcal{O}_{N^{-}}$on $A$;

- $C$ is a level- $N^{+}$structure, that is, a subgroup scheme of $A\left[N^{+}\right]$of order $\left(N^{+}\right)^{2}$ that is stable and cyclic under the action of $\mathcal{O}_{N^{-}}$.

Suppose that $N^{+}$is neat in the sense that $(A, \iota, C)$ has no non-trivial automorphism. Then the functor $y_{N^{+}, N^{-}}^{\natural}$ is represented by a scheme $y_{N^{+}, N^{-}}^{\natural}$, quasi-projective over $\mathbb{Z}\left[1 / N^{+}\right]$, projective if and only if $N^{-}>1$. We denote by $y_{N^{+}, N^{-}}$the canonical compactification of $y_{N^{+}, N^{-}}$(only necessary when $N^{-}=1$ ). It is a regular scheme and smooth over $\mathbb{Z}\left[1 / N^{+} N^{-}\right]$.

Notation 2.10. Put $Y_{N^{+}, N^{-}}=y_{N^{+}, N^{-} ; \mathbb{Q}}$ and denote its Jacobian by $J_{N^{+}, N^{-}}$. For each prime $\ell \mid N^{-}$, we denote by $\Phi_{N^{+}, N^{-}}^{(\ell)}$ the group of connected components of the special fiber of the Néron model of $J_{N^{+}, N^{-} ; \mathbb{Q}_{\ell^{2}}}$ over $\mathbb{Z}_{\ell^{2}}$.

2.3.1. Hecke algebra and cohomology. Let $d$ be a factor of $N^{+}$, we have degeneracy morphisms

$$
\delta_{N^{+}, N^{-}}^{\left(d, d^{\prime}\right.}: y_{N^{+}, N^{-}} \rightarrow y_{N^{+} / d, N^{-}}
$$

indexed by positive integers $d^{\prime} \mid d$ such that $\delta_{N^{+}, N^{-}}^{(d, 1)}$ is the natural projection by forgetting the level structure. For each prime $v \nmid N^{+} N^{-}$, we have the Hecke correspondence $T_{N^{+}, N^{-}}^{(v)} \in \operatorname{Corr}\left(Y_{N^{+}, N^{-}}\right)$as

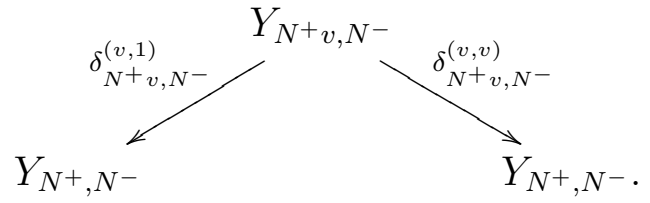

Denote by $\mathbb{T}_{N^{+}, N^{-}}^{\text {sph,rat }}$ the (commutative) $\mathbb{Q}$-subalgebra of $\operatorname{Corr}\left(Y_{N^{+}, N^{-}}\right)$generated by $T_{N^{+}, N^{-}}^{(v)}$ for all primes $v \nmid N^{+} N^{-}$. Denote by $\mathbb{T}_{N^{+}, N^{-}}^{\mathrm{sph}}$ the image of $\mathbb{T}_{N^{+}, N^{-}}^{\text {sph,rat }}$ under the homomorphism $\operatorname{cor}_{\mathrm{dR}}$, which is a finite-dimensional commutative $\mathbb{Q}$-algebra. For each $\operatorname{Gal}(\mathbb{C} / \mathbb{Q})$-conjugacy class $\sigma$ of irreducible cuspidal automorphic representations of $\mathrm{GL}_{2}(\mathbb{A})$ that occur in $\mathrm{H}_{\mathrm{dR}}^{1}\left(Y_{N^{+}, N^{-}}\right)$, we have a unique idempotent $\mathscr{P}_{\sigma}^{\mathrm{dR}} \in \mathbb{T}_{N^{+}, N^{-}}^{\mathrm{sph}}$ such that it induces the natural projection $\oplus \mathrm{H}_{\mathrm{dR}}^{*}\left(Y_{N^{+}, N^{-}}\right) \rightarrow \mathrm{H}_{\mathrm{dR}}^{1}\left(Y_{N^{+}, N^{-}}\right)[\sigma]$ onto the $\sigma$-isotypic subspace $\mathrm{H}_{\mathrm{dR}}^{1}\left(Y_{N^{+}, N^{-}}\right)[\sigma]$ of $\mathrm{H}_{\mathrm{dR}}^{1}\left(Y_{N^{+}, N^{-}}\right)$.

Definition 2.11. A $\sigma$-projector (of level $N^{+}$) is an element $\mathscr{P}_{\sigma}$ in $\mathbb{T}_{N^{+}, N^{-}}^{\mathrm{sph} \text { sat }}$ such that $\operatorname{cor}_{\mathrm{dR}}\left(\mathscr{P}_{\sigma}\right)=\mathscr{P}_{\sigma}^{\mathrm{dR}}$.

Consider the $p$-adic étale cohomology $\mathrm{H}_{\text {ét }}^{1}\left(Y_{N^{+}, N^{-} ; \mathbb{Q}^{\text {ac }}}, \mathbb{Q}_{p}(1)\right)$ as a $p$-adic representation of $\Gamma_{\mathbb{Q}}$. Denote by $\mathrm{H}_{\sigma}^{1}\left(Y_{N^{+}, N^{-} ; \mathbb{Q}^{\text {ac }}}\right)$ its $\sigma$-isotypic subspace which is preserved under the action of $\Gamma_{\mathbb{Q}}$. Then $\mathscr{P}_{\sigma}^{\mathrm{dR}}$ induces a natural projection

$$
\mathscr{P}_{\sigma}^{\text {ét }}: \mathrm{H}_{\text {ét }}^{1}\left(Y_{N^{+}, N^{-} ; \mathbb{Q}^{\text {ac }}}, \mathbb{Q}_{p}(1)\right) \rightarrow \mathrm{H}_{\sigma}^{1}\left(Y_{N^{+}, N^{-}} ; \mathbb{Q}^{\text {ac }}\right),
$$

by the Comparison Theorem between de Rham and étale cohomology. It is $\Gamma_{\mathbb{Q}^{-}}$ equivariant. 
2.3.2. Good reduction at $\ell \nmid N^{-}$. Suppose that $\ell$ is a prime not dividing $N^{+} N^{-}$. Denote by $y_{N^{+}, N^{-} ; \mathbb{F}_{\ell^{2}}}$ the supersingular locus of $y_{N^{+}, N^{-} ; \mathbb{F}_{\ell^{2}}}$, which is preserved under the action of $\mathrm{Fr}_{\ell}$.

Proposition 2.12. The scheme $\mathrm{y}_{N^{+}, N^{-} ; \mathbb{F}_{\ell^{2}}}$ is a disjoint union of Spec $\mathbb{F}_{\ell^{2}}$. There is a canonical bijection between $\mathrm{y}_{N^{+}, N^{-} ; \mathbb{F}_{\ell^{2}}}\left(\mathbb{F}_{\ell^{2}}\right)$ and $\mathcal{T}_{N^{+}, N^{-\ell}}$, under which $\mathrm{Fr}_{\ell}$ acts by $\mathrm{op}_{\ell}$.

Proof. The first statement is well-known. The second statement is proved in [Rib89, Theorem 3.4].

For later use, we briefly recall how to construct the map $\underset{N^{+}, N^{-} ; \mathbb{F}_{\ell^{2}}}{\text { ss }}\left(\mathbb{F}_{\ell^{2}}\right) \rightarrow \mathcal{T}_{N^{+}, N^{-} \ell}$ in the case $N^{+}=1$. Suppose that $(A, \iota)$ is an element in $y_{1, N^{-} ; \mathbb{F}_{\ell^{2}}}\left(\mathbb{F}_{\ell^{2}}\right)$. The (maximal) Eichler order in $B_{N^{-} \ell}$ would be $\operatorname{End}(A, \iota)$, that is, the algebra of all endomorphisms commuting with $\iota\left(\mathcal{O}_{N^{-}}\right)$. We only need to specify the orientation $o_{v}: \operatorname{End}(A, \iota) \rightarrow \mathbb{F}_{v^{2}}$ for every prime $v$ dividing $N^{-} \ell$. There are two cases.

- When $v \neq \ell$, we let $\mathfrak{m}_{v}$ be the maximal ideal of $\operatorname{End}(A, \iota)$ of residue characteristic $v$. Then the action of $\operatorname{End}(A, \iota) / \mathfrak{m}_{v}$ on $A\left[\mathfrak{m}_{v}\right]\left(\mathbb{F}_{\ell}^{\mathrm{ac}}\right)$, which is an $\mathbb{F}_{v^{2} \text {-vectors }}$ space of dimension 1, induces a homomorphism $\operatorname{End}(A, \iota) \rightarrow \mathbb{F}_{v^{2}}$. We set this homomorphism to be $o_{v}$.

- When $v=\ell$, consider the action of $\operatorname{End}(A, \iota)$ on the $\mathbb{F}_{\ell^{2} \text {-vector space }}$ Lie $A$, which is in fact a scalar action, and hence induces an orientation $o_{\ell}: \operatorname{End}(A, \iota) \rightarrow \mathbb{F}_{\ell^{2}}$.

Remark 2.13. By construction, the map $y_{N^{+}, N^{-} ; \mathbb{F}_{\ell^{2}}}\left(\mathbb{F}_{\ell^{2}}\right) \rightarrow y_{N^{+} / d, N^{-} ; \mathbb{F}_{\ell^{2}}}\left(\mathbb{F}_{\ell^{2}}\right)$ induced by the degeneracy morphism $\delta_{N^{+}, N^{-}}^{\left(d, d^{\prime}\right)}: y_{N^{+}, N^{-}} \rightarrow y_{N^{+} / d, N^{-}}$coincides with $\delta_{N^{+}, N^{-} \ell}^{\left(d, d^{\prime}\right)}: \mathcal{T}_{N^{+}, N^{-} \ell} \rightarrow \mathcal{T}_{N^{+} / d, N^{-} \ell}($ see Notation $2.2(5))$.

2.3.3. Bad reduction at $\ell \mid N^{-}$. Suppose that $\ell$ divides $N^{-}$. In particular, the scheme $y_{N^{+}, N^{-}}=y_{N^{+}, N^{-}}$is proper. For simplicity and also for the later application, we further assume that $\ell$ is odd and inert in $F$. Recall that we have always fixed an embedding $O_{F} \hookrightarrow \mathcal{O}_{N^{-}}$.

For each geometric point $s \in y_{N^{+}, N^{-} ; \mathbb{F}_{\ell}}(k)$, denote the associated abelian variety by $A_{s}$. Denote by $\tau(s)$ the multi-set of characters of $O_{F} / \ell$ appearing in its action on the Dieudonné module of $A_{s}[\ell]_{\alpha}$. There are three possibilities for $\tau(s):\left\{\tau_{\ell}^{\bullet}\right\},\left\{\tau_{\ell}^{\circ}\right\}$ and $\left\{\tau_{\ell}^{\bullet}, \tau_{\ell}^{\circ}\right\}$.

Denote by $y_{N^{+}, N^{-} ; \mathbb{F}_{\ell^{2}}}$ (resp. $y_{N^{+}, N^{-} ; \mathbb{F}_{\ell^{2}}}^{\circ}$ ) the union of irreducible components of $y_{N^{+}, N^{-;} ; \mathbb{F}_{\ell^{2}}}$ whose generic geometric point $s$ satisfies $\tau(s)=\left\{\tau_{\ell}^{\bullet}\right\}$ (resp. $\tau(s)=\left\{\tau_{\ell}^{\circ}\right\}$ ). Denote by $y_{N^{+}, N^{-} ; \mathbb{F}_{\ell^{2}}}^{\text {ssp }}$ the scheme theoretical intersection of $y_{N^{+}, N^{-} ; \mathbb{F}_{\ell^{2}}}$ and $y_{N^{+}, N^{-} ; \mathbb{F}_{\ell^{2}}}$. Note that $y_{N^{+}, N^{-} ; \mathbb{F}_{\ell^{2}}}^{\mathrm{ssp}}$ classifies mixed and exceptional data in the terminology of [Rib89].

Proposition 2.14. We have that

(1) $y_{N^{+}, N^{-} ; \mathbb{F}_{\ell^{2}}}$ and $y_{N^{+}, N^{-} ; \mathbb{F}_{\ell^{2}}}$ intersect transversally at $y_{N^{+}, N^{-} ; \mathbb{F}_{\ell^{2}}}^{\mathrm{ssp}}$;

(2) $\mathrm{y}_{N^{+}, N^{-} ; \mathbb{F}_{\ell^{2}}}^{\mathrm{ssp}}$ is a disjoint union of $\operatorname{Spec} \mathbb{F}_{\ell^{2}}$, canonically indexed by $\mathcal{T}_{N^{+} \ell, N^{-} / \ell}$, under which $\mathrm{Fr}_{\ell}$ acts by $\mathrm{op}_{\ell}$; 
(3) both $y_{N^{+}, N^{-} ; \mathbb{F}_{\ell^{2}}}^{\bullet}$ and $y_{N^{+}, N^{-} ; \mathbb{F}_{\ell^{2}}}^{\circ}$ are disjoint union of $\mathbf{P}_{\mathbb{F}_{\ell^{2}}}^{1}$, each canonically indexed by $\mathcal{T}_{N^{+}, N^{-} / \ell}$, under which $\mathrm{Fr}_{\ell}$ acts by switching two factors.

Proof. Part (1) is a consequence of Čerednik-Drinfeld uniformization. The remaining parts are proved in [Rib89, Theorem $4.15 \& 4.16, \S 5]$.

\section{Remark 2.15.}

(1) In order to distinguish between different orientations, when we identify the set $\pi_{0}\left(y_{N^{+}, N^{-} ; \mathbb{F}_{\ell^{2}}}\right)$ (resp. $\pi_{0}\left(y_{N^{+}, N^{-} ; \mathbb{F}_{\ell^{2}}}^{\circ}\right)$ with $\mathcal{T}_{N^{+}, N^{-} / \ell}$, we will use the notation $\mathcal{T}_{N^{+}, N^{-} / \ell}^{\bullet}\left(\operatorname{resp} . \mathcal{T}_{N^{+}, N^{-} / \ell}^{\circ}\right)$. As a set, it is nothing but $\mathcal{T}_{N^{+}, N^{-} / \ell}$.

(2) The natural map

$$
\begin{aligned}
& y_{N^{+}, N^{-} ; \mathbb{F}_{\ell^{2}}}^{\operatorname{ssp}}\left(\mathbb{F}_{\ell^{2}}\right) \rightarrow \pi_{0}\left(y_{N^{+}, N^{-} ; \mathbb{F}_{\ell^{2}}}\right) \\
& \text { resp. } y_{N^{+}, N^{-} ; \mathbb{F}_{\ell^{2}}}\left(\mathbb{F}_{\ell^{2}}\right) \rightarrow \pi_{0}\left(y_{N^{+}, N^{-} ; \mathbb{F}_{\ell^{2}}}^{\circ}\right)
\end{aligned}
$$

is given by $\delta_{N^{+} \ell, N^{-} / \ell}^{(\ell, 1)}\left(\operatorname{resp} . \delta_{N^{+} \ell, N^{-} / \ell}^{(\ell, \ell)}\right)$; see Notation $2.2(5)$.

(3) For $?=\bullet, \circ$, the map $\pi_{0}\left(y_{N^{+}, N^{-} ; \mathbb{F}_{\ell^{2}}}\right) \rightarrow \pi_{0}\left(y_{N^{+} / d, N^{-} ; \mathbb{F}_{\ell^{2}}}\right)$ induced by the degeneracy morphism $\delta_{N^{+}, N^{-}}^{\left(d, d^{\prime}\right)}: y_{N^{+}, N^{-}} \rightarrow y_{N^{+} / d, N^{-}}$coincides with the map

$$
\delta_{N^{+}, N^{-} / \ell}^{\left(d, d^{\prime}\right.}: \mathcal{T}_{N^{+}, N^{-} / \ell} \rightarrow \mathcal{T}_{N^{+} / d, N^{-} \ell}
$$

See Notation $2.2(5)$.

2.4. Hilbert modular surfaces. We first introduce the Hilbert modular scheme for $F$. The details of the construction can be found in [Rap78, §1] or [Gor02, Chapter 3]. Let $\mathfrak{M}$ be an ideal of $O_{F}$.

For each representative $\left(\mathfrak{a}, \mathfrak{a}^{+}\right) \in \mathrm{Cl}(F)^{+}$, denote by $\underline{\widetilde{X}}_{\mathfrak{M}}^{\left(\mathfrak{a}, \mathfrak{a}^{+}\right)}$the functor from the category of schemes over $\mathbb{Z}\left[1 / \mathrm{Nm}_{F / \mathbb{Q}} \mathfrak{M} \cdot \operatorname{disc} F\right]$ to the category of groupoids such that $\underline{\tilde{X}}_{\mathfrak{M}}^{\left(\mathfrak{a}, \mathfrak{a}^{+}\right)}(S)$ is the groupoid of quadruples $(A, \iota, \psi, C)$, where

- $A$ is an $S$-abelian scheme of relative dimension 2 ;

- $\iota$ is an $O_{F}$-multiplication on $A$, that is, a homomorphism $\iota: O_{F} \rightarrow \operatorname{End}(A)$ of $\mathbb{Z}$ algebras satisfying the Rapoport condition: locally over $S$, the $O_{F} \otimes_{\mathbb{Z}} \mathscr{O}_{S}$-module Lie $A$ is free of rank 1 ;

- $\psi:\left(\mathfrak{P}_{A}, \mathfrak{P}_{A}^{+}\right) \stackrel{\sim}{\rightarrow}\left(\mathfrak{a}, \mathfrak{a}^{+}\right)$, where $\mathfrak{P}_{A}=\operatorname{Hom}_{O_{F}}\left(A, A^{\vee}\right)^{\text {symm }}$ is the $O_{F}$-module of symmetric homomorphisms from $A$ to $A^{\vee}$, and $\mathfrak{P}_{A}^{+} \subset \mathfrak{P}_{A}$ is the subset of polarizations;

- $C$ is a level- $\mathfrak{M}$ structure, that is, a subgroup scheme of $A[\mathfrak{M}]$ of order $\mathrm{Nm}_{F / \mathbb{Q}} \mathfrak{M}$ that is stable and cyclic under the action of $O_{F}$.

Suppose that $\mathfrak{M}$ is neat in the sense that the triple $(A, \iota, C)$ has no non-trivial automorphism. Then the functor $\underline{\widetilde{X}}_{\mathfrak{M}}^{\left(\mathfrak{a}, \mathfrak{a}^{+}\right)}$is represented by a scheme $\widetilde{X}_{\mathfrak{M}}^{\left(\mathfrak{a}, \mathfrak{a}^{+}\right)}$, smooth and quasi-projective over $\mathbb{Z}\left[1 / \mathrm{Nm}_{F / \mathbb{Q}} \mathfrak{M} \cdot \operatorname{disc}(F)\right]$. Put

$$
\widetilde{X}_{\mathfrak{M}}^{\natural}=\bigsqcup_{\mathrm{Cl}(F)^{+}} \widetilde{X}_{\mathfrak{M}}^{\left(\mathfrak{a}, \mathfrak{a}^{+}\right)}
$$


which is known as the Hilbert modular scheme associated to $F$ of level-M $\mathfrak{M}$ structure. Put $U_{F}=O_{F}^{\times,+} / O_{F}^{\times, 2}$, whose cardinality is at most 2 . The finite group $U_{F}$ acts on $\tilde{\mathscr{X}}_{\mathfrak{M}}^{\left(\mathfrak{a}, \mathfrak{a}^{+}\right)}$ by sending $(A, \iota, \psi, C)$ to $(A, \iota, \psi \circ u, C)$ for $u \in U_{F}$. Denote by $X_{\mathfrak{M}}^{\left(\mathfrak{a}, \mathfrak{a}^{+}\right)}$the quotient of $\widetilde{X}_{\mathfrak{M}}^{\left(\mathfrak{a}, \mathfrak{a}^{+}\right)}$by $U_{F}$ under this (free, since $\mathfrak{M}$ is neat) action, which is an algebraic space. Put

$$
X_{\mathfrak{M}}^{\natural}=\bigsqcup_{\mathrm{Cl}(F)^{+}} X_{\mathfrak{M}}^{\left(\mathfrak{a}, \mathfrak{a}^{+}\right)} .
$$

2.4.1. Relation with Shimura varieties. Put $\mathbf{G}=\operatorname{Res}_{F / \mathbb{Q}} \mathrm{GL}_{2, F}$ as a reductive group over $\mathbb{Q}$. Define $\widetilde{\mathbf{G}}$ as the pullback in the following diagram

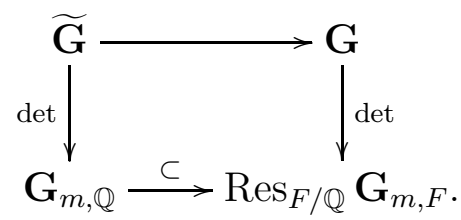

Denote by $\mathbf{K}_{\mathfrak{M}}$ the open compact subgroup of $\mathbf{G}\left(\mathbb{A}^{\infty}\right)$ corresponding to the level- $\mathfrak{M}$ structure, and $\widetilde{\mathbf{K}}_{\mathfrak{M}}$ its intersection with $\widetilde{\mathbf{G}}\left(\mathbb{A}^{\infty}\right)$. We have the natural morphism of canonical models (over $\mathbb{Q}$ ) of Shimura varieties

$$
\operatorname{Sh}\left(\widetilde{\mathbf{G}}, \widetilde{\mathbf{K}}_{\mathfrak{M}}\right)_{\mathbb{Q}} \rightarrow \operatorname{Sh}\left(\mathbf{G}, \mathbf{K}_{\mathfrak{M}}\right)_{\mathbb{Q}},
$$

whose complex analytification is identified with the following well-known double-coset expression

$$
\widetilde{\mathbf{G}}(\mathbb{Q}) \backslash(\mathbb{C}-\mathbb{R})^{2} \times \widetilde{\mathbf{G}}\left(\mathbb{A}^{\infty}\right) / \widetilde{\mathbf{K}}_{\mathfrak{M}} \rightarrow \mathbf{G}(\mathbb{Q}) \backslash(\mathbb{C}-\mathbb{R})^{2} \times \mathbf{G}\left(\mathbb{A}^{\infty}\right) / \mathbf{K}_{\mathfrak{M}} .
$$

The scheme $\tilde{X}_{\mathfrak{M}}^{\left(\mathfrak{D}^{-1},\left(\mathfrak{D}^{-1}\right)^{+}\right)}$is an integral canonical model of the Shimura variety $\operatorname{Sh}\left(\widetilde{\mathbf{G}}, \widetilde{\mathbf{K}}_{\mathfrak{M}}\right)_{\mathbb{Q}}$ over $\mathbb{Z}\left[1 / \mathrm{Nm}_{F / \mathbb{Q}} \mathfrak{M} \cdot \operatorname{disc}(F)\right]$ (see [Rap78, Theorem 1.28]). By abuse of notation, denote by $\operatorname{Sh}\left(\mathbf{G}, \mathbf{K}_{\mathfrak{M}}\right)$ the integral canonical model of $\operatorname{Sh}\left(\mathbf{G}, \mathbf{K}_{\mathfrak{M}}\right)_{\mathbb{Q}}$ over $\mathbb{Z}\left[1 / \mathrm{Nm}_{F / \mathbb{Q}} \mathfrak{M} \cdot \operatorname{disc}(F)\right]$. Therefore, (2.1) extends uniquely to a morphism

$$
\tilde{X}_{\mathfrak{M}}^{\left(\mathfrak{D}^{-1},\left(\mathfrak{D}^{-1}\right)^{+}\right)} \rightarrow \operatorname{Sh}\left(\mathbf{G}, \mathbf{K}_{\mathfrak{M}}\right) .
$$

In fact, we have a morphism

$$
\widetilde{X}_{\mathfrak{M}}^{\natural} \rightarrow \operatorname{Sh}\left(\mathbf{G}, \mathbf{K}_{\mathfrak{M}}\right) .
$$

Lemma 2.16. The morphism (2.4) factorizes through $X_{\mathfrak{M}}^{\natural}$, and the induced morphism $X_{\mathfrak{M}}^{\natural} \rightarrow \operatorname{Sh}\left(\mathbf{G}, \mathbf{K}_{\mathfrak{M}}\right)$ is an isomorphism. In particular, the scheme $X_{\mathfrak{M}}^{\natural}$ is smooth over $\mathbb{Z}\left[1 / \mathrm{Nm}_{F / \mathbb{Q}} \mathfrak{M} \cdot \operatorname{disc}(F)\right]$.

Proof. It suffices to check that (2.3) factorizes through $\mathcal{X}_{\mathfrak{M}}^{\left(\mathfrak{D}^{-1},\left(\mathfrak{D}^{-1}\right)^{+}\right)}$and the induced morphism $X_{\mathfrak{M}}^{\left(\mathfrak{P}^{-1},\left(\mathfrak{D}^{-1}\right)^{+}\right)} \rightarrow \operatorname{Sh}\left(\mathbf{G}, \mathbf{K}_{\mathfrak{M}}\right)$ is an open and closed immersion.

For the first part, by canonicality, we only need to check on the generic fiber. For this, we only need to check for $\mathbb{C}$-points. Then the claim follows since (2.2) is simply the quotient by $U_{F}$ onto its image (see [Gor02, Theorem 2.17, Remark 2.18]).

For the second part, it suffices to show that for each prime $\ell \nmid \mathrm{Nm}_{F / \mathbb{Q}} \mathfrak{M} \cdot \operatorname{disc}(F)$, the morphism $X_{\mathfrak{M} ; \mathbb{Z}_{(\ell)}}^{\left(\mathfrak{D}^{-1}\left(\mathfrak{D}^{-1}\right)^{+}\right)} \rightarrow \operatorname{Sh}\left(\mathbf{G}, \mathbf{K}_{\mathfrak{M}}\right)_{\mathbb{Z}_{(\ell)}}$ is an open and closed immersion. We 
note that $\widetilde{\mathbf{G}}$ and $\mathbf{G}$ have the same connected Shimura data. In particular, the induced morphism of neutral connected components of $\widetilde{X}_{\mathfrak{M} ; \mathrm{W}\left(\mathbb{F}_{\ell}^{\text {ac }}\right)}^{\left(\mathfrak{D}^{-1}\right)}$ and $\operatorname{Sh}\left(\mathbf{G}, \mathbf{K}_{\mathfrak{M}}\right)_{\mathrm{W}\left(\mathbb{F}_{\ell}^{\text {ac }}\right)}$ is an isomorphism. Then the base change of (2.3) to $\mathrm{W}\left(\mathbb{F}_{\ell}^{\mathrm{ac}}\right)$ is finite étale, of degree $\# U_{F}$.

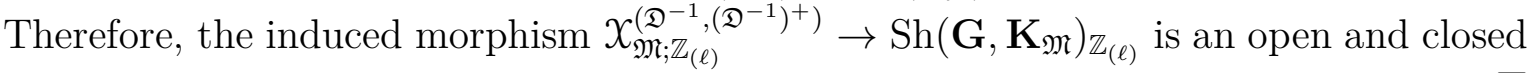
immersion.

Remark 2.17. Let $k$ be an algebraically closed field. The set $\chi_{\mathfrak{M}}^{\natural}(k)$ corresponds canonically to the set of isomorphism classes of triples $(A, \iota, C)$ where $A$ is a $k$-abelian surface, $\iota$ is an $O_{F}$-multiplication, and $C$ is a level- $\mathfrak{M}$ structure.

Notation 2.18. Denote by $X_{\mathfrak{M}}^{\natural}=X_{\mathfrak{M} ; \mathbb{Q}}^{\natural}$ the generic fiber of $X_{\mathfrak{M}}^{\natural}$. Let $X_{\mathfrak{M}}$ be the minimal resolution of the Baily-Borel compactification of $X_{\mathfrak{M}}^{\natural}$. It is a smooth projective surface over $\mathbb{Q}$ and we have $\pi_{0}\left(X_{\mathfrak{M}}\right) \simeq \mathrm{Cl}(F)^{+}$.

\subsubsection{Hecke algebra and cohomology.}

Lemma 2.19. The singular cohomology group $\mathrm{H}^{3}\left(X_{\mathfrak{M}}(\mathbb{C}), \mathbb{Z}\right)$ vanishes.

Proof. This follows from [vdG88, Theorem 6.1] and the Poincaré duality for compact manifolds.

For ideals $\mathfrak{d}^{\prime}|\mathfrak{d}| \mathfrak{M}$ of $O_{F}$, we have degeneracy morphisms

$$
\gamma_{\mathfrak{M}}^{\left(\mathfrak{d}, \mathfrak{d}^{\prime}\right)}: X_{\mathfrak{M}} \rightarrow X_{\mathfrak{M} / \mathfrak{d}}
$$

such that $\gamma_{\mathfrak{M}}^{(\mathfrak{d}, 1)}$ is the natural projection by forgetting the level structure. For each finite place $\mathfrak{v}$ of $F$ that is coprime to $\operatorname{Nm}_{F / \mathbb{Q}} \mathfrak{M} \cdot \operatorname{disc}(F)$, we have the Hecke correspondence $S_{\mathfrak{M}}^{(\mathfrak{v})} \in \operatorname{Corr}\left(X_{\mathfrak{M}}\right)$ as

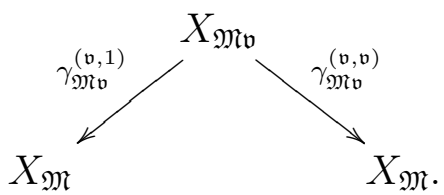

Denote by $\mathbb{S}_{\mathfrak{M}}^{\text {sph,rat }} \subset \operatorname{Corr}\left(X_{\mathfrak{M}}\right)$ the (commutative) $\mathbb{Q}$-subalgebra generated by $S_{\mathfrak{M}}^{(\mathfrak{v})}$ for all places $\mathfrak{v}$ that are coprime to $\operatorname{Nm}_{F / \mathbb{Q}} \mathfrak{M} \cdot \operatorname{disc}(F)$. Denote by $\mathbb{S}_{\mathfrak{M}}^{\mathrm{sph}, \mathrm{dR}}$ the image of $\mathbb{S}_{\mathfrak{M}}^{\mathrm{sph}}$,rat under the homomorphism $\operatorname{cor}_{\mathrm{dR}}$, which is a finite-dimensional commutative $\mathbb{Q}$ algebra. For each $\operatorname{Gal}(\mathbb{C} / \mathbb{Q})$-conjugacy class $\pi$ of irreducible cuspidal automorphic representations of $\operatorname{Res}_{F / \mathbb{Q}} \mathrm{GL}_{2}(\mathbb{A})$ that occurs in $\mathrm{H}_{\mathrm{dR}}^{2}\left(X_{\mathfrak{M}}\right)$, we have a unique idempotent $\mathscr{P}_{\pi}^{\mathrm{dR}} \in \mathbb{S}_{\mathfrak{M}}^{\mathrm{sph}, \mathrm{dR}}$ such that it induces the natural projection $\oplus \mathrm{H}_{\mathrm{dR}}^{*}\left(X_{\mathfrak{M}}\right) \rightarrow \mathrm{H}_{\mathrm{dR}}^{2}\left(X_{\mathfrak{M}}\right)[\pi]$ onto the $\pi$-isotypic subspace $\mathrm{H}_{\mathrm{dR}}^{2}\left(X_{\mathfrak{M}}\right)[\pi]$ of $\mathrm{H}_{\mathrm{dR}}^{2}\left(X_{\mathfrak{M}}\right)$.

Definition 2.20. A $\pi$-projector (of level $\mathfrak{M}$ ) is an element $\mathscr{P}_{\pi}$ in $\mathbb{S}_{\mathfrak{M}}^{\text {sph,rat }}$ such that $\operatorname{cor}_{\mathrm{dR}}\left(\mathscr{P}_{\pi}\right)=\mathscr{P}_{\pi}^{\mathrm{dR}}$.

Consider the $p$-adic étale cohomology $\mathrm{H}_{\text {ét }}^{2}\left(X_{\mathfrak{M} ; \mathbb{Q}^{\text {ac }}}, \mathbb{Q}_{p}(1)\right)$ as a $p$-adic representation of $\Gamma_{\mathbb{Q}}$. Denote by $\mathrm{H}_{\pi}^{2}\left(Y_{\mathfrak{M} ; \mathbb{Q}^{a c}}\right)$ its $\pi$-isotypic subspace which is preserved under the action of $\Gamma_{\mathbb{Q}}$. Then $\mathscr{P}_{\pi}^{\mathrm{dR}}$ induces a natural projection

$$
\mathscr{P}_{\pi}^{\text {ét }}: \mathrm{H}_{\text {ét }}^{2}\left(X_{\mathfrak{M} ; \mathbb{Q}^{\mathrm{ac}}}, \mathbb{Q}_{p}(1)\right) \rightarrow \mathrm{H}_{\pi}^{2}\left(X_{\mathfrak{M} ; \mathbb{Q}^{\mathrm{ac}}}\right)
$$


by the Comparison Theorem between de Rham and étale cohomology. It is $\Gamma_{\mathbb{Q}^{-}}$ equivariant.

2.4.3. Supersingular locus. Let $\ell$ be a prime not dividing $2 \mathfrak{M}$ and inert in $F$. We recall some constructions in [GO00].

For each geometry point $s \in X_{\mathfrak{M} ; \mathbb{F}_{\ell}}^{\natural}(k)$, denote the associated abelian variety by $A_{s}$. As in the curve case, denote by $\tau(s)$ the multi-set of characters of $O_{F} / \ell$ appearing in its action on the Dieudonné module of $A_{s}[\ell]_{\alpha}$. Now there are four possibilities for $\tau(s)$ : $\emptyset,\left\{\tau_{\ell}^{\bullet}\right\},\left\{\tau_{\ell}^{\circ}\right\}$ and $\left\{\tau_{\ell}^{\bullet}, \tau_{\ell}^{\circ}\right\}$.

Denote by $X_{\mathfrak{M} ; \mathbb{F}_{\ell^{2}}}^{\mathrm{ssp}}\left(\right.$ resp. $\left.X_{\mathfrak{M} ; \mathbb{F}_{\ell^{2}}}^{\bullet}, X_{\mathfrak{M} ; \mathbb{F}_{\ell^{2}}}^{\circ}\right)$ the closed subscheme of $X_{\mathfrak{M} ; \mathbb{F}_{\ell^{2}}}^{\natural}$, with the reduced induced structure, having the property that for every geometric point $s$ of it, the set $\tau(s)$ contains $\left\{\tau_{\ell}^{\bullet}, \tau_{\ell}^{\circ}\right\}$ (resp. $\left\{\tau_{\ell}^{\bullet}\right\},\left\{\tau_{\ell}^{\circ}\right\}$ ).

Proposition 2.21. We have that

(1) $X_{\mathfrak{M} ; \mathbb{F}_{\ell^{2}}}^{\bullet}$ and $X_{\mathfrak{M} ; \mathbb{F}_{\ell^{2}}}^{\circ}$ intersect transversally at $X_{\mathfrak{M} ; \mathbb{F}_{\ell^{2}}}^{\mathrm{ssp}}$;

(2) $X_{\mathfrak{M} ; \mathbb{F}_{\ell^{2}}}^{\mathrm{ssp}}$ is a disjoint union of $\mathrm{Spec} \mathbb{F}_{\ell^{2}}$, canonically indexed by $\mathcal{S}_{\mathfrak{M} \ell}$, under which $\mathrm{Fr}_{\ell}$ acts by $\mathrm{op}_{\ell}$

(3) both $X_{\mathfrak{M} ; \mathbb{F}_{\ell^{2}}}^{\bullet}$ and $X_{\mathfrak{M} ; \mathbb{F}_{\ell^{2}}}^{\circ}$ are disjoint unions of $\mathbf{P}_{\mathbb{F}_{\ell^{2}}}^{1}$, each canonically indexed by $\mathcal{S}_{\mathfrak{M}}$, under which $\mathrm{Fr}_{\ell}$ acts by switching the two sets and preserving values in $\mathcal{S}_{\mathfrak{M}}$;

(4) the self-intersection number (inside $X_{\mathfrak{M} ; \mathbb{F}_{\ell^{2}}}$ ) of each irreducible component of $X_{\mathfrak{M} ; \mathbb{F}_{\ell^{2}}}^{\bullet}$ or $X_{\mathfrak{M} ; \mathbb{F}_{\ell^{2}}}^{\circ}$ is $-2 \ell$.

Proof. Part (1) is proved in [GO00, Corollary 2.3.7]. Part (2) follows from Proposition 2.9, since $X_{\mathfrak{M} ; \mathbb{F}_{\ell^{2}}}^{\mathrm{ssp}}$ is of dimension 0 and parameterizes superspecial mixed triples $(A, \iota, C)$.

For (3), take an algebraically closed field $k$ containing $\mathbb{F}_{\ell^{2}}$. By [BG99, Theorem 5.1], every irreducible component of $X_{\mathfrak{M} ; k}^{\bullet}\left(\operatorname{resp} . X_{\mathfrak{M} ; k}^{\circ}\right)$ is the base of a Moret-Bailly family associated to a $\bullet$-pure (resp. o-pure) triple $(A, \iota, C)$, unique up to isomorphism, over $k$. Thus by Proposition 2.21, both $X_{\mathfrak{M} ; k}^{\bullet}$ and $X_{\mathfrak{M} ; k}^{\circ}$ are disjoint unions of $\mathbf{P}_{k}^{1}$, each canonically indexed by $\mathcal{S}_{\mathfrak{M}}$. It is clear from the construction that $\operatorname{Fr}_{\ell}$ acts by switching the two factors. In particular, the Frobenius element $\mathrm{Fr}_{\ell^{2}}$ fixes each connected component of $\mathcal{X}_{\mathfrak{M} ; k}^{\bullet}$ and $\mathcal{X}_{\mathfrak{M} ; k}^{\circ}$. Since each component contains an $\mathbb{F}_{\ell^{2}}$-point, it is isomorphic to $\mathbf{P}_{\mathbb{F}_{\ell 2}}^{1}$.

Part (4) is proved in $[\mathrm{Nic00}, \S 3.0 .2]$, which is also a very special case of the main calculation in the work of Tian-Xiao (see [TX14, §1.1]), in terms of Lemma 2.16.

Remark 2.22 .

(1) In order to distinguish between different orientations, when we identify the set $\pi_{0}\left(X_{\mathfrak{M} ; \mathbb{F}_{\ell^{2}}}^{\bullet}\right)$ (resp. $\left.\pi_{0}\left(X_{\mathfrak{M} ; \mathbb{F}_{\ell^{2}}}^{\circ}\right)\right)$ with $\mathcal{S}_{\mathfrak{M}}$, we use the notation $\mathcal{S}_{\mathfrak{M}}^{\bullet}$ (resp. $\left.\mathcal{S}_{\mathfrak{M}}^{\circ}\right)$. As a set, it is nothing but $\mathcal{S}_{\mathfrak{M}}$.

(2) By Remark 2.8, the natural map

$$
\begin{aligned}
X_{\mathfrak{M} ; \mathbb{F}_{\ell^{2}}}^{\mathrm{ssp}}\left(\mathbb{F}_{\ell^{2}}\right) \rightarrow \pi_{0}\left(X_{\mathfrak{M} ; \mathbb{F}_{\ell^{2}}}^{\bullet}\right) \\
\text { resp. } X_{\mathfrak{M} ; \mathbb{F}_{\ell^{2}}}^{\text {ssp }}\left(\mathbb{F}_{\ell^{2}}\right) \rightarrow \pi_{0}\left(X_{\mathfrak{M} ; \mathbb{F}_{\ell^{2}}}^{\circ}\right)
\end{aligned}
$$

is given by $\gamma_{\mathfrak{M} \ell}^{(\ell, 1)}$ (resp. $\gamma_{\mathfrak{M} \ell}^{(\ell, \ell)}$ ); see Notation $2.3(2)$. 
(3) Define $X_{\mathfrak{M} ; \mathbb{F}_{\ell^{2}}}^{\mathrm{ss}}$ to be the union of $X_{\mathfrak{M} ; \mathbb{F}_{\ell^{2}}}^{\bullet}$ and $X_{\mathfrak{M} ; \mathbb{F}_{\ell^{2}}}^{\circ}$. It is a proper subscheme and coincides with the supersingular locus of $\chi_{\mathfrak{M} ; \mathbb{F}_{\ell^{2}}}^{\natural}$.

(4) Similar parametrization in Proposition $2.21(2,3)$ is also obtained by Tian and Xiao [TX13, §1.5.1], where they use the language of Shimura sets, that is, certain double quotients of $\left(Q \otimes_{\mathbb{Z}} \widehat{\mathbb{Z}}\right)^{\times}$. However, their parametrization is not canonical, and the canonicality in our parametrization is crucial in this article since we need to use it for different primes. See Propositions 2.24 and 2.25.

2.5. Hirzebruch-Zagier morphisms. Now we assume that all prime factors of $N^{-}$ are inert in $F$. In what follows, we will view $y_{N^{+}, N^{-}}$and $y_{N^{+}, N^{-}}$as schemes over $\mathbb{Z}\left[1 / N^{+} \operatorname{disc}(F)\right]$. There is a natural composite morphism

$$
\zeta_{N^{+}, N^{-}}^{\natural}: y_{N^{+}, N^{-}}^{\natural} \rightarrow \widetilde{x}_{N^{+}}^{\natural} \rightarrow x_{N^{+}}^{\natural}
$$

in which the first morphism sends $(A, \iota, C)$ to $\left(A,\left.\iota\right|_{O_{F}}, \psi_{D}, C\right)$ where $\psi_{D}$ is the canonical principal polarization associated to $\left(\mathcal{O}_{N^{-}}, *\right)$ (Notation $\left.2.2(3)\right)$. The morphism $\zeta_{N^{+}, N^{-}}^{\natural}$

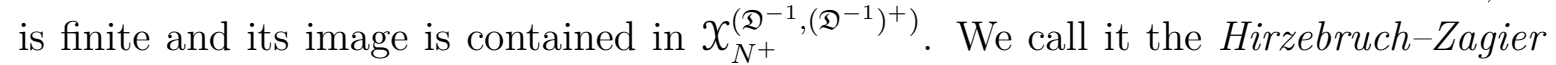
morphism, whose generic fiber is an example of special morphisms of Shimura varieties in the sense that it is induced by embedding of Shimura data.

Let $\mathcal{D}_{N^{+}}>0$ be the least square-free integer such that

- it is divisible by all prime factors of $N^{+} \operatorname{disc}(F)$;

- $X_{N^{+}}$extends to a projective smooth scheme $X_{N^{+}}$over $\mathbb{Z}\left[1 / \mathcal{D}_{N^{+}}\right]$such that the morphism $Y_{N^{+}, 1} \rightarrow X_{N^{+}}$extends to a finite morphism $y_{N^{+}, 1 ; \mathbb{Z}\left[1 / \mathcal{D}_{N^{+}}\right]} \rightarrow x_{N^{+}}$ whose restriction to $y_{N^{+}, 1 ; \mathbb{Z}\left[1 / \mathcal{D}_{N^{+}}\right]}$coincides with $\zeta_{N^{+}, 1 ; \mathbb{Z}\left[1 / \mathcal{D}_{N^{+}}\right]}^{\natural}$.

Therefore, we always have a (unique) morphism

$$
\zeta_{N^{+}, N^{-}}: y_{N^{+}, N^{-} ;} \mathbb{Z}\left[1 / \mathcal{D}_{N^{+}}\right] \rightarrow x_{N^{+}}
$$

over $\mathbb{Z}\left[1 / \mathcal{D}_{N^{+}}\right]$extending $\zeta_{N^{+}, N^{-}}^{\natural}$. In what follows, $y_{N^{+}, N^{-}}$will automatically be regarded as a scheme over $\mathbb{Z}\left[1 / \mathcal{D}_{N^{+}}\right]$and by abuse of notation, all kinds of base change of $\zeta_{N^{+}, N^{-}}$will still be denoted by $\zeta_{N^{+}, N^{-}}$. Although here we use the same notation as in Notation 2.3, no confusion will arise since $\zeta_{N^{+}, N^{-}}$is a morphism of schemes (resp. map of sets) if and only if $\wp\left(N^{-}\right)$is even (resp. odd).

Put $z_{N^{+}, N^{-}}=y_{N^{+}, N^{-}} \times_{\operatorname{Spec} \mathbb{Z}\left[1 / \mathcal{D}_{N^{+}}\right]} x_{N^{+}}$. We have two projection morphisms

$$
y_{N^{+}, N^{-}} \stackrel{\pi_{y}}{\longleftarrow} z_{N^{+}, N^{-}} \stackrel{\pi_{x}}{\longrightarrow} x_{N^{+}} .
$$

Denote by $\boldsymbol{\Delta}_{N^{+}, N^{-}}$the graph of $\zeta_{N^{+}, N^{-}}$, which is a codimension 2 cycle on $z_{N^{+}, N^{-}}$. Put $Z_{N^{+}, N^{-}}=Z_{N^{+}, N^{-} ; \mathbb{Q}}$.

2.5.1. Case of proper intersection. Let $\ell$ be a prime not dividing $2 N^{-} \mathcal{D}_{N^{+}}$and inert in $F$.

Lemma 2.23. Denote by $\mathcal{J}$ the scheme-theoretical intersection of the closed subschemes $\boldsymbol{\Delta}_{N^{+}, N^{-} ; \mathbb{F}_{\ell^{2}}}$ and $\pi_{x}^{-1} \chi_{N^{+} ; \mathbb{F}_{\ell^{2}}}^{\mathrm{ss}}$ of $z_{N^{+}, N^{-} ; \mathbb{F}_{\ell^{2}}}$. Then $\pi_{y} \mathcal{J}$ (resp. $\left.\pi_{X} \mathcal{J}\right)$ is contained in the supersingular locus $\mathrm{y}_{N^{+}, N^{-} ; \mathbb{F}_{\ell^{2}}}^{\text {ss }}$ (resp. superspecial locus $X_{N^{+} ; \mathbb{F}_{\ell^{2}}}^{\mathrm{ssp}}$ ), and $\mathcal{J}$ is a disjoint union of $\operatorname{Spec} \mathbb{F}_{\ell^{2}}$. 
Proof. It is obvious that $\pi_{y} \mathcal{J}$ is contained in $y_{N^{+}, N^{-} ; \mathbb{F}_{\ell^{2}}}$. Suppose that $s \in \mathcal{J}(k)$ is a geometric point, which corresponds to the data $\left(A_{s}, \iota_{s}, C_{s}\right) \in y_{N^{+}, N^{-} ; \mathbb{F}_{\ell^{2}}}(k)$. If $A_{s}$ is ordinary, then $\pi_{X}(s)$ does not locate in $X_{N^{+} ; \mathbb{F}_{\ell^{2}}}^{\mathrm{Ss}}$, which is a contradiction. Therefore, the abelian surface $A_{s}$ is supersingular and hence superspecial. This means that $\pi_{x}(s) \in$ $X_{N^{+} ; \mathbb{F}_{\ell^{2}}}^{\mathrm{ssp}}(k)$. Thus, The first claim follows. In particular, $\mathcal{J}$ is of dimension 0 .

For the second claim, we only need to show that $\boldsymbol{\Delta}_{N^{+}, N^{-} ; \mathbb{F}_{\ell^{2}}}$ and $\pi_{x}^{-1} X_{N^{+} ; \mathbb{F}_{\ell^{2}}}^{\mathrm{ss}}$ intersect transversally. We use displays of $p$-divisible groups (in the version developed in [Zin02]). Since $\ell$ is odd, we may choose an element $\delta \in \mathrm{W}\left(\mathbb{F}_{\ell}^{\text {ac }}\right)$ such that $\delta^{2} \in \mathbb{Z}_{\ell}^{\times}$and we have an isomorphism $O_{F, \ell} \stackrel{\sim}{\rightarrow} \mathbb{Z}_{\ell}[\delta]$ whose composition with the natural inclusion $\mathbb{Z}_{\ell}[\delta] \subset$ $\mathrm{W}\left(\mathbb{F}_{\ell}^{\text {ac }}\right)$ gives $\tau_{\ell}^{\bullet}$. Then $\tau_{\ell}^{\circ}(\delta)=-\delta$. Choose an isomorphism $\mathcal{O}_{N^{-}} \otimes_{\mathbb{Z}} \mathbb{Z}_{\ell} \simeq \operatorname{Mat}_{2}\left(\mathbb{Z}_{\ell}\right)$ such that

- the inclusion $O_{F, \ell} \hookrightarrow \mathcal{O}_{N^{-}} \otimes_{\mathbb{Z}} \mathbb{Z}_{\ell}$ sends $x+y \delta$ to $\left(\begin{array}{cc}x & y \delta^{2} \\ y & x\end{array}\right)$;

- the involution $*$ sends $\left(\begin{array}{ll}a & b \\ c & d\end{array}\right)$ to $\left(\begin{array}{cc}a & c \delta^{2} \\ b \delta^{-2} & d\end{array}\right)$.

Since the problem is local, we pick up an arbitrary $\mathbb{F}_{\ell}^{\text {ac }}$-point $s \in \mathcal{J}\left(\mathbb{F}_{\ell}^{\text {ac }}\right)$ and consider only the abelian surface $A_{s}$. Suppose that the $\ell$-divisible group $A_{s}\left[\ell^{\infty}\right]=G^{\oplus 2}$, and $G$ is displayed by the matrix $\left(\begin{array}{ll}a & b \\ c & d\end{array}\right)$ under the basis $e_{1}, e_{2}$ such that the polarization is given by the standard symplectic matrix $\left(\begin{array}{c}1 \\ -1\end{array}\right)$. By the theory of displays, the universal deformation ring of $G$ is isomorphic to $R_{0}=\mathbb{F}_{\ell}^{\text {ac }}\left[\left[t_{0}\right]\right]$, and the universal deformation is displayed by the matrix $\left(\begin{array}{cc}a+c T_{0} & b+d T_{0} \\ c & d\end{array}\right)$, where $T_{0} \in \mathrm{W}\left(R_{0}\right)$ is the Teichmüller lift of $t_{0}$. Now $A_{s}\left[\ell^{\infty}\right]$ is displayed by the matrix

$$
\left(\begin{array}{llll}
a & & b & \\
& a & & b \\
c & & d & \\
& c & & d
\end{array}\right),
$$

with the (principal) polarization given by the symplectic matrix

$$
\left(\begin{array}{llll} 
& & 1 & \\
& & & \delta \\
-1 & & & \\
& -\delta & &
\end{array}\right)
$$

The universal deformation ring of $A_{s}\left[\ell^{\infty}\right]$ is isomorphic to $R=\mathbb{F}_{\ell}^{\text {ac }}\left[\left[t_{11}, t_{12}, t_{21}, t_{22}\right]\right]$, and the universal deformation is displayed by the matrix

$$
\left(\begin{array}{cccc}
a+c T_{11} & c T_{12} & b+d T_{11} & d T_{12} \\
c T_{21} & a+c T_{22} & d T_{21} & b+d T_{22} \\
c & & d & \\
& c & & d
\end{array}\right),
$$


where $T_{i j} \in \mathrm{W}(R)$ is the Teichmüller lift of $t_{i j}$. We have that

- the universal deformation ring of $A_{s}\left[\ell^{\infty}\right]$ with the above given polarization is cut out by the ideal generated by $t_{12}-\delta^{2} t_{21}$;

- the universal deformation ring of $A_{s}\left[\ell^{\infty}\right]$ with the $O_{F, \ell^{-}}$action and the above given polarization is cut out by the ideal generated by $\left\{t_{11}-t_{22}, t_{12}-\delta^{2} t_{21}\right\}$;

- the universal deformation ring of $A_{s}\left[\ell^{\infty}\right]$ with the $\operatorname{Mat}_{2}\left(\mathbb{Z}_{\ell}\right)$-action and the above given polarization is cut out by the ideal generated by $\left\{t_{11}-t_{22}, t_{12}, t_{21}\right\}$.

Moreover, by the computation in [GO00, Theorem 2.3.4], the universal deformation ring of $A_{s}\left[\ell^{\infty}\right]$ with the $O_{F, \ell^{-}}$action and the above given polarization along $X_{N^{+} ; \mathbb{F}_{\ell}^{\text {ac }}}$ is cut out by the ideal generated by $\left\{t_{11}-t_{22}, t_{12}-\delta^{2} t_{21}, t_{11}-\delta t_{21}\right\}$. Therefore, the local ring of $\boldsymbol{\Delta}_{N^{+}, N^{-} ; \mathbb{F}_{\ell^{2}}} \times z_{N^{+}, N^{-} ; \mathbb{F}_{\ell}} \pi_{X}^{-1} \chi_{N^{+} ; \mathbb{F}_{\ell^{2}}}^{\bullet}$ at $s$ is isomorphic to

$$
\mathbb{F}_{\ell}^{\mathrm{ac}}\left[\left[t_{0}, t_{11}, t_{12}, t_{21}, t_{22}\right]\right] /\left\langle t_{0}-t_{11}, t_{11}-t_{22}, t_{12}, t_{21}, t_{11}-\delta t_{21}\right\rangle,
$$

which is isomorphic to $\mathbb{F}_{\ell}^{\text {ac }}$. This implies that $\boldsymbol{\Delta}_{N^{+}, N^{-} ; \mathbb{F}_{\ell^{2}}}$ and $\pi_{x}^{-1} \chi_{N^{+} ; \mathbb{F}_{\ell}}$ intersect transversally. We have a similar argument for $X_{N^{+} ; \mathbb{F}_{\ell^{2}}}^{\circ}$. The lemma then follows.

Proposition 2.24. The restriction of the map $\zeta_{N^{+}, N^{-}}\left(\mathbb{F}_{\ell^{2}}\right)$ to $y_{N^{+}, N^{-} ; \mathbb{F}_{\ell^{2}}}\left(\mathbb{F}_{\ell^{2}}\right)=$ $\mathcal{T}_{N^{+}, N^{-} \ell}$ takes values in the subset $\mathcal{X}_{N^{+} ; \mathbb{F}_{\ell^{2}}}^{\text {ssp }}\left(\mathbb{F}_{\ell^{2}}\right)$. If we compose it with the natural map from $X_{N^{+} ; \mathbb{F}_{\ell^{2}}}^{\text {ssp }}\left(\mathbb{F}_{\ell^{2}}\right)$ to $\pi_{0}\left(X_{N^{+} ; \mathbb{F}_{\ell^{2}}}^{\bullet}\right)=\mathcal{S}_{N^{+}}^{\bullet}\left(\right.$ resp. $\left.\pi_{0}\left(X_{N^{+} ; \mathbb{F}_{\ell^{2}}}^{\circ}\right)=\mathcal{S}_{N^{+}}^{\circ}\right)$, then the resulting map is equal to

$$
\begin{gathered}
\mathcal{T}_{N^{+}, N^{-} \ell} \stackrel{\zeta_{N^{+}, N^{-} \ell}}{\longrightarrow} \mathcal{S}_{N^{+}}=\mathcal{S}_{N^{+}}^{\bullet}, \\
\operatorname{resp.} \mathcal{T}_{N^{+}, N^{-} \ell} \stackrel{\zeta_{N^{+}, N^{-}} \ell^{\circ o p_{\ell}}}{\longrightarrow} \mathcal{S}_{N^{+}}=\mathcal{S}_{N^{+}}^{\circ}
\end{gathered}
$$

Proof. We prove the first case, by which the second one will follow. Suppose that $k$ is an algebraically closed field containing $\mathbb{F}_{\ell^{2}}$, and $(A, \iota, C)$ is a triple in $y_{N^{+}, N^{-} ; \mathbb{F}_{\ell^{2}}}(k)$, which implies that $\left(A,\left.\iota\right|_{O_{F}}, C\right)$ is superspecial mixed. We only need to show that if $R=\operatorname{End}(A, \iota, C)$ with the natural orientation as in the proof of Proposition 2.12, then $\operatorname{End}\left(A / H_{\bullet},\left.\iota\right|_{O_{F}}, C\right)$ is the order $R_{\sharp}$ as in Definition 2.4. Note that $R \otimes_{\mathbb{Z}} O_{F}$ is naturally contained in $\operatorname{End}\left(A / H_{\bullet},\left.\iota\right|_{O_{F}}, C\right)$.

It amounts to show that for every prime $v \mid N^{-} \ell$, the $\left(R / v, O_{F} / v\right)$-bimodule $\operatorname{End}\left(A / H_{\bullet},\left.\iota\right|_{O_{F}}, C\right) \otimes_{\mathbb{Z}} \mathbb{Z}_{v} / R \otimes_{\mathbb{Z}} O_{F, v} \simeq \mathbb{F}_{v^{2}}$ is isomorphic to $o_{v} \otimes \tau_{v}^{\bullet}$. When $v=\ell$, it holds since $O_{F} / \ell$ acts on the Dieudonné module of $H_{\bullet}$ by $\tau_{\ell}^{\bullet}$. When $v \mid N^{-}$, the question is local. We identify $O_{F, v}$ with $\mathbb{Z}_{v^{2}}$ via $\tau_{v}^{\bullet}$, and $\mathcal{O}_{N^{-}, v}:=\mathcal{O}_{N^{-}} \otimes_{\mathbb{Z}} \mathbb{Z}_{v}$ with $\mathbb{Z}_{v^{2}} \oplus \mathbb{Z}_{v^{2}}[\Pi]$ such that $\Pi^{2}=v$ and $a \Pi=\Pi a^{\theta}$ for $a \in \mathbb{Z}_{v^{2}}$. We identify $\mathrm{T}_{v}(A)$ with $\mathcal{O}_{N^{-}, v}$ such that $\iota$ acts by left multiplication. In particular, the algebra $R_{v} \simeq \mathcal{O}_{N^{-}, v}$ acts on $\mathrm{T}_{v}(A)$ via right multiplication. The algebra $\operatorname{End}\left(A / H_{\bullet},\left.\iota\right|_{O_{F}}, C\right)_{v}$ is identified with the centralizer of $\mathbb{Z}_{v^{2}}$ in $\operatorname{End}_{\mathbb{Z}_{v}}\left(\mathrm{~T}_{v}(A)\right)$, where the latter is identified with $\operatorname{Mat}_{2}\left(\mathbb{Z}_{v^{2}}\right)$ under the basis $\{1, \Pi\}$. Under the same basis, the suborder $R_{v} \otimes_{\mathbb{Z}_{v}} \mathbb{Z}_{v^{2}}$ is identified with

$$
\left\{\left(\begin{array}{ll}
a & b \\
c & d
\end{array}\right) \in \operatorname{Mat}_{2}\left(\mathbb{Z}_{v^{2}}\right) \mid c \in v \mathbb{Z}_{v^{2}}\right\}
$$


Moreover, the action of $a \otimes b \in \mathbb{Z}_{v^{2}} \otimes_{\mathbb{Z}_{v}} \mathbb{Z}_{v^{2}} \subset R_{v} \otimes_{\mathbb{Z}_{v}} \mathbb{Z}_{v^{2}}$ on $\operatorname{End}\left(A / H_{\bullet},\left.\iota\right|_{O_{F}}, C\right)_{v}$ is given by

$$
x \in \operatorname{Mat}_{2}\left(\mathbb{Z}_{v^{2}}\right) \mapsto\left(\begin{array}{ll}
b & \\
& b
\end{array}\right) x\left(\begin{array}{cc}
a & \\
& a^{\theta}
\end{array}\right) .
$$

This implies that the bi-action of $\left(R / v, O_{F} / v\right)$ on $\operatorname{End}\left(A / H_{\bullet},\left.\iota\right|_{O_{F}}, C\right) \otimes_{\mathbb{Z}} \mathbb{Z}_{v} / R \otimes_{\mathbb{Z}} O_{F, v}$ is $o_{v} \otimes \tau_{v}^{\bullet}$.

2.5.2. Case of improper intersection. Let $\ell$ be a prime that divides $N^{-}$but not $2 \mathcal{D}_{N^{+}}$, and is inert in $F$.

Proposition 2.25. We have that

(1) The restriction of the map $\zeta_{N^{+}, N^{-}}\left(\mathbb{F}_{\ell^{2}}\right)$ to $y_{N^{+}, N^{-} ; \mathbb{F}_{\ell^{2}}}^{\mathrm{ssp}}\left(\mathbb{F}_{\ell^{2}}\right)$ takes values in $X_{N^{+} ; \mathbb{F}_{\ell^{2}}}^{\text {ssp }}\left(\mathbb{F}_{\ell^{2}}\right)$. Regarded as a map from $\mathcal{T}_{N^{+} \ell, N^{-} / \ell}$ to $\mathcal{S}_{N^{+} \ell}$, it is equal to

$$
\mathcal{T}_{N^{+} \ell, N^{-} / \ell} \stackrel{\zeta_{N^{+} \ell, N^{-}} / \ell}{\longrightarrow} \mathcal{S}_{N^{+} \ell}
$$

(2) For $?=\bullet, \circ$, the restriction of the morphism $\zeta_{N^{+}, N^{-}}$to $y_{N^{+}, N^{-} ; \mathbb{F}_{\ell^{2}}}$ has image

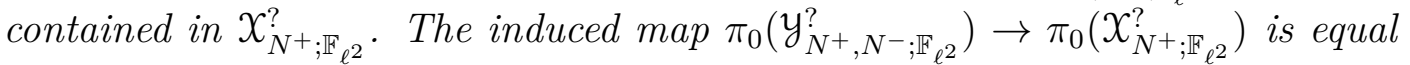
to

$$
\mathcal{T}_{N^{+}, N^{-} / \ell} \stackrel{\zeta_{N^{+}, N^{-}} / \ell}{\longrightarrow} \mathcal{S}_{N^{+}}
$$

Proof. We first prove (2), for $?=\bullet$ and the other is similar. As before, we fix an algebraically closed field $k$ containing $\mathbb{F}_{\ell^{2}}$. By [Rib89, §5], there is a bijection between $\pi_{0}\left(y_{N^{+}, N^{-} ; \mathbb{F}_{\ell^{2}}}\right)$ and the set of isomorphism classes of triples $(A, \iota, C)$ over $k$ such that $\left(A,\left.\iota\right|_{O_{F}}, C\right)$ is $\bullet$-pure. By the same proof of Proposition 2.24, we know that if $R=$ $\operatorname{End}(A, \iota, C)$ with the natural orientation as in the proof of Proposition 2.12, then $\operatorname{End}\left(A,\left.\iota\right|_{O_{F}}, C\right)$, which naturally contains $R \otimes_{\mathbb{Z}} O_{F}$, is the order $R_{\sharp}$ as in Definition 2.4. In particular, (2) follows.

Part (1) is proved in the same way as (2), with the only extra work of the orientation at $\ell$. But this follows from (2), Remark 2.15 (2) and Remark 2.22 (2).

Remark 2.26. The above proposition together with its proof amounts to say that the natural commutative diagram

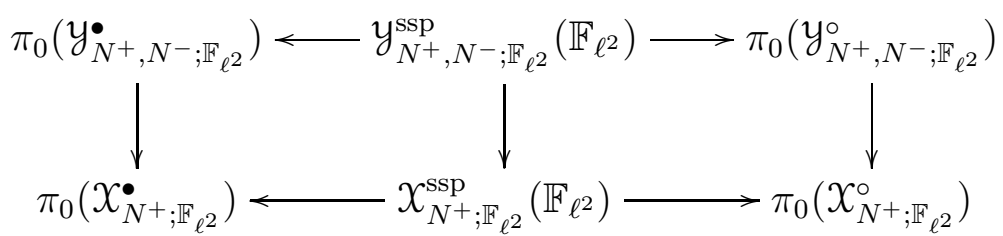

is canonically identified with

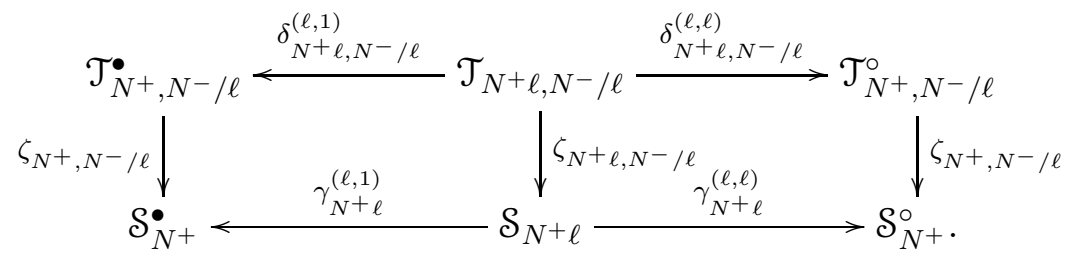




\section{Construction of COHOMOlogy Classes}

In this chapter, we construct various cohomology classes from Hirzebruch-Zagier cycles. In §3.1, we recall the definition of Bloch-Kato Selmer groups of general Galois modules with various coefficients, and collect some facts for later use. In $\$ 3.2$, we set up our main theorem in the language of automorphic representations, and list assumptions which will be needed at various stages in the later discussion. In $\S 3.3$, we introduce Hirzebruch-Zagier cycles in details, from which we construct the very first class in the Selmer group with rational coefficient. The main theorem in the language of automorphic representations will be stated at the end of this section. In $\S 3.4$, we fix suitable projections, which are related to non-vanishing testing vectors of local invariant forms. In §3.5, we collect some important facts from Bertolini-Darmon [BD05], with slight modification to fit our need. In §3.6, we construct variants of Hirzebruch-Zagier classes with torsion coefficients, as candidates for annihilators of Selmer groups. In particular, we introduce the notion of strongly admissible primes.

3.1. Bloch-Kato Selmer group. We recall the definition of Bloch-Kato Selmer groups for Galois representations with various coefficients. For simplicity, our number field will be $\mathbb{Q}$, and $p$ is odd according to our convention.

Let $(\rho, \mathrm{V})$ be a $p$-adic representation of $\Gamma_{\mathbb{Q}}$, that is, as we recall $\mathrm{V}$ is a finite dimensional $\mathbb{Q}_{p}$-vector space and

$$
\rho: \Gamma_{\mathbb{Q}} \rightarrow \mathrm{GL}(\mathrm{V})
$$

is a homomorphism, continuous with respect to the profinite (resp. p-adic) topology on the source (resp. target). Define

$$
\mathrm{H}_{f}^{1}\left(\mathbb{Q}_{p}, \mathrm{~V}\right)=\operatorname{Ker}\left[\mathrm{H}^{1}\left(\mathbb{Q}_{p}, \mathrm{~V}\right) \rightarrow \mathrm{H}^{1}\left(\mathbb{Q}_{p}, \mathrm{~V} \otimes_{\mathbb{Q}_{p}} \mathrm{~B}_{\text {cris }}\right)\right]
$$

It is known that if $\mathrm{V}$ is crystalline, then elements in $\mathrm{H}_{f}^{1}\left(\mathbb{Q}_{p}, \mathrm{~V}\right)$ correspond exactly to those extensions

$$
0 \rightarrow \mathrm{V} \rightarrow \mathrm{V}_{1} \rightarrow \mathbb{Q}_{p} \rightarrow 0
$$

in which $\mathrm{V}_{1}$ is also crystalline.

For each finite set $\Sigma$ of places of $\mathbb{Q}$ containing $\{p, \infty\}$, denote by $\mathbb{Q}^{\Sigma}$ the maximal subfield of $\mathbb{Q}^{\text {ac }}$ that is unramified outside of $\Sigma$, and $\Gamma_{\mathbb{Q}, \Sigma}=\operatorname{Gal}\left(\mathbb{Q}^{\Sigma} / \mathbb{Q}\right)$ which is a quotient group of $\Gamma_{\mathbb{Q}}$.

Definition 3.1 (Bloch-Kato Selmer group, [BK90]). Suppose that there exists a finite set $\Sigma$ of places of $\mathbb{Q}$ containing $\{p, \infty\}$ such that $\mathrm{V}$ is unramified outside $\Sigma$. We define the Bloch-Kato Selmer group $\mathrm{H}_{f}^{1}(\mathbb{Q}, \mathrm{V})$ of $\mathrm{V}$ as the subspace of $\mathrm{H}^{1}(\mathbb{Q}, \mathrm{V})$ consisting of $s$ such that

- $s$ belongs to the subspace $\mathrm{H}^{1}\left(\Gamma_{\mathbb{Q}, \Sigma}, \mathrm{V}\right)$;

- $\operatorname{loc}_{v}(s) \in \mathrm{H}_{\mathrm{unr}}^{1}\left(\mathbb{Q}_{v}, \mathrm{~V}\right)$ for all primes $v \nmid p$; and

- $\operatorname{loc}_{p}(s) \in \mathrm{H}_{f}^{1}\left(\mathbb{Q}_{p}, \mathrm{~V}\right)$.

It is clear that $\mathrm{H}_{f}^{1}(\mathbb{Q}, \mathrm{V})$ does not depend on the choice of $\Sigma$. If $\mathrm{T} \subset \mathrm{V}$ is a stable lattice, then we have a natural map $\mathrm{H}^{1}(\mathbb{Q}, \mathrm{T}) \rightarrow \mathrm{H}^{1}(\mathbb{Q}, \mathrm{V})$ and define the Bloch-Kato Selmer group $\mathrm{H}_{f}^{1}(\mathbb{Q}, \mathrm{T})$ of $\mathrm{T}$ to be the inverse image of $\mathrm{H}_{f}^{1}(\mathbb{Q}, \mathrm{V})$ under the above map. 
For each integer $n \geq 1$, define $\mathrm{H}_{f}^{1}\left(\mathbb{Q}, \overline{\mathrm{T}}^{n}\right)$ to be the image of $\mathrm{H}_{f}^{1}(\mathbb{Q}, \mathrm{T})$ under the natural $\operatorname{map} \mathrm{H}^{1}(\mathbb{Q}, \mathrm{T}) \rightarrow \mathrm{H}^{1}\left(\mathbb{Q}, \overline{\mathrm{T}}^{n}\right)$.

Remark 3.2. By [Mil06, Corollary 4.15] and [Tat76, Corollary 2.1], the groups $\mathrm{H}^{1}\left(\Gamma_{\mathbb{Q}, \Sigma}, \mathrm{T}\right)$ and hence $\mathrm{H}_{f}^{1}(\mathbb{Q}, \mathrm{T}), \mathrm{H}_{f}^{1}\left(\mathbb{Q}, \overline{\mathrm{T}}^{n}\right)$ are all finitely generated $\mathbb{Z}_{p}$-modules.

Definition 3.3. We say that a $p$-adic representation $\mathrm{V}$ of $\Gamma_{\mathbb{Q}}$ is tamely pure if for all primes $v \nmid p, \mathrm{H}^{1}\left(\mathbb{Q}_{v}, \mathrm{~V}\right)=0$. If $\mathrm{V}$ is tamely pure, then $s \in \mathrm{H}_{f}^{1}(\mathbb{Q}, \mathrm{V})$ if and only if $\operatorname{loc}_{p}(s) \in \mathrm{H}_{f}^{1}\left(\mathbb{Q}_{p}, \mathrm{~V}\right)$.

Lemma 3.4. Let $\mathrm{T}$ be a stable lattice in a tamely pure $p$-adic representation $\mathrm{V}$ of $\Gamma_{\mathbb{Q}}$. Then for each prime $v \nmid p$ such that $\mathrm{I}_{v}$ acts trivially on $\mathrm{V}$, the image of the reduction map $\mathrm{H}^{1}\left(\mathbb{Q}_{v}, \mathrm{~T}\right) \rightarrow \mathrm{H}^{1}\left(\mathbb{Q}_{v}, \overline{\mathrm{T}}^{n}\right)$ is contained in $\mathrm{H}_{\mathrm{unr}}^{1}\left(\mathbb{Q}_{v}, \overline{\mathrm{T}}^{n}\right)$ for every integer $n \geq 1$.

Proof. The short exact sequence $0 \rightarrow \mathrm{T} \rightarrow \mathrm{V} \rightarrow \mathrm{V} / \mathrm{T} \rightarrow 0$ induces the following commutative diagram

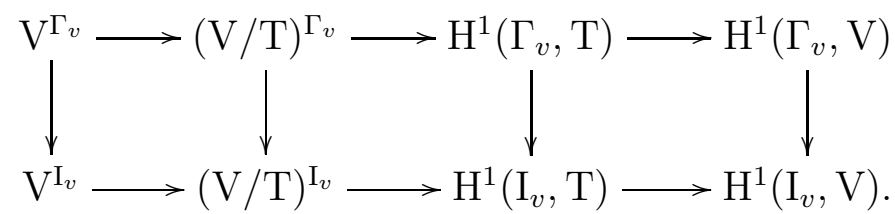

Since $\mathrm{I}_{v}$ acts trivially on $\mathrm{T}$, the arrow $\mathrm{V}^{\mathrm{I}_{v}} \rightarrow(\mathrm{V} / \mathrm{T})^{\mathrm{I}_{v}}$ is surjective, which implies that $\mathrm{H}^{1}\left(\mathrm{I}_{v}, \mathrm{~T}\right) \rightarrow \mathrm{H}^{1}\left(\mathrm{I}_{v}, \mathrm{~V}\right)$ is injective. Since $\mathrm{H}^{1}\left(\Gamma_{v}, \mathrm{~V}\right)=0$, the $\operatorname{map~} \mathrm{H}^{1}\left(\Gamma_{v}, \mathrm{~T}\right) \rightarrow \mathrm{H}^{1}\left(\mathrm{I}_{v}, \mathrm{~T}\right)$ is trivial. The lemma follows immediately.

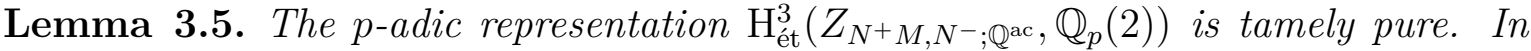
other words, for each prime $v \nmid p$, we have $\mathrm{H}^{1}\left(\mathbb{Q}_{v}, \mathrm{H}_{\text {ét }}^{3}\left(Z_{N^{+} M, N^{-} ; \mathbb{Q}^{\text {ac }}}, \mathbb{Q}_{p}(2)\right)\right)=0$.

This lemma actually can be avoided for the proof of main theorems (see Lemma 3.9). However, we include it here as a typical example for tamely pure representations.

Proof. The lemma follows from the purity of monodromy filtration for $Z_{N^{+} M, N^{-} ; \mathbb{Q}_{v}}$, which is known. In fact, since $Z_{N^{+} M, N^{-} ; \mathbb{Q}_{v}}$ is the product of a smooth projective curve and a smooth projective surface over $\mathbb{Q}_{v}$, the purity follows from that of the latter two by Künneth formula and [Del80, (1.6.9)]. The case for curves is proved in [SGA7I, Exposé IX], while the case of surfaces is proved by Rapoport-Zink [RZ82] together with de Jong's alteration [dJ96].

3.2. Twisted triple product Galois representation. We now switch to the language of automorphic representations. Let $\sigma$ (resp. $\pi$ ) be an irreducible cuspidal automorphic representation of $\mathrm{GL}_{2}(\mathbb{A})$ (resp. $\operatorname{Res}_{F / \mathbb{Q}} \mathrm{GL}_{2}(\mathbb{A})$ ) defined over $\mathbb{Q}$, of weight 2 (resp. $(2,2)$ ) and trivial central character, with conductor $N$ (resp. $\mathfrak{M}$ ). We also assume that they are both non-dihedral.

Let $p$ be an (odd) prime, which will be fixed. We may associate to $\sigma$ (resp. $\pi$ ) a $p$-adic representation $\rho_{\sigma}: \Gamma_{\mathbb{Q}} \rightarrow \mathrm{GL}\left(\mathrm{V}_{\sigma}\right)$ (resp. $\left.\rho_{\pi}: \Gamma_{F} \rightarrow \mathrm{GL}\left(\mathrm{V}_{\pi}\right)\right)$ of $\Gamma_{\mathbb{Q}}\left(\operatorname{resp} . \Gamma_{F}\right)$ of dimension 2 . 
Notation 3.6. Put

$$
{ }^{\sharp} \rho_{\pi}=\left(\otimes \operatorname{Ind}_{\Gamma_{F}}^{\Gamma_{\mathbb{Q}}} \rho_{\pi}\right)(-1)
$$

as the tensor induction (with the Tate twist), with the underlying $\mathbb{Q}_{p}$-vector space ${ }^{\sharp} \mathrm{V}_{\pi}$ which is isomorphic to $\mathrm{V}_{\pi}^{\otimes 2}$. It is isomorphic to the Asai representation As $\rho_{\pi}$ associated to $\rho_{\pi}$ (with the Tate twist).

There is a perfect $\Gamma_{\mathbb{Q}^{-}}$equivariant symmetric bilinear pairing ${ }^{\sharp} \mathrm{V}_{\pi} \times{ }^{\sharp} \mathrm{V}_{\pi} \rightarrow \mathbb{Q}_{p}$ coming from the construction. Denote by $\mathrm{O}\left({ }^{\sharp} \mathrm{V}_{\pi}\right)$ the orthogonal group defined by the above pairing. Then we have a homomorphism

$$
{ }^{\sharp} \rho_{\pi}: \Gamma_{\mathbb{Q}} \rightarrow \mathrm{O}\left({ }^{\sharp} \mathrm{V}_{\pi}\right) .
$$

Finally, put $\left(\rho_{\sigma, \pi}, \mathrm{V}_{\sigma, \pi}\right)=\left(\rho_{\sigma}, \mathrm{V}_{\sigma}\right) \otimes_{\mathbb{Q}_{p}}\left({ }^{\sharp} \rho_{\pi},{ }^{\sharp} \mathrm{V}_{\pi}\right)$.

3.2.1. Asai-decomposable case. Recall that $\pi$ has trivial central character and is nondihedral.

Definition 3.7. We say that $\pi$ is Asai-decomposable if $\pi^{\theta} \simeq \pi \otimes \omega$ for some (necessarily quadratic) automorphic character $\omega$ of $\operatorname{Res}_{F / \mathbb{Q}} \mathbf{G}_{m, F}(\mathbb{A})$, where $\pi^{\theta}=\pi \circ \theta$.

If $\pi$ is Asai-decomposable, then we have a decomposition

$$
{ }^{\sharp} \mathrm{V}_{\pi}=\mathrm{V}_{\breve{\pi}}(-1) \oplus \mathbb{Q}_{p}(\breve{\eta})
$$

of $p$-adic representations of $\Gamma_{\mathbb{Q}}$, where the latter two direct summands are both absolutely irreducible. Here, $\breve{\eta}$ is a Dirichlet character of $\Gamma_{\mathbb{Q}}$. If we denote by $\omega_{\breve{\eta}}$ the associated automorphic character of $\mathbb{A}^{\times}$via the global class field theory, then $\omega_{\breve{\eta}} \circ \mathrm{Nm}_{F / \mathbb{Q}}=\omega$. By [LR98, Theorem 1], the restricted character $\left.\omega\right|_{\mathbb{A}} \times$ is trivial. Therefore, the characters $\omega_{\breve{\eta}}$ and hence $\breve{\eta}$ are either trivial or quadratic. Denote by $\breve{F} \subset \mathbb{Q}^{\text {ac }}$ the splitting field of $\breve{\eta}$, which is either $\mathbb{Q}$ or a quadratic field unramified outside $\operatorname{Nm}_{F / \mathbb{Q}} \mathfrak{M} \cdot \operatorname{disc}(F)$.

$\operatorname{Put}\left(\rho_{\sigma \times \breve{\pi}}, \mathrm{V}_{\sigma \times \breve{\pi}}\right)=\mathrm{V}_{\sigma} \otimes_{\mathbb{Q}_{p}} \mathrm{~V}_{\breve{\pi}}(-1)$ and $\left(\rho_{\sigma \otimes \breve{\eta}}, \mathrm{V}_{\sigma \otimes \breve{\eta}}\right)=\mathrm{V}_{\sigma} \otimes_{\mathbb{Q}_{p}} \mathbb{Q}_{p}(\breve{\eta})$. We adopt the convention that $\breve{\eta}$ is the trivial character if $\pi$ is not Asai-decomposable.

3.2.2. Assumptions. We write $N=N^{+} N^{-}$such that $N^{+}$(resp. $N^{-}$) is the product of prime factors that are split (resp. inert) in $F$. Fix an integer $M \geq 1$ such that $\mathfrak{M} \mid M$.

Assumption 3.8 (Group R). We introduce the following assumptions on $\sigma, \pi$ and $p$. (R1): $N^{-}$is square-free; $p, N, M \operatorname{disc}(F)$ are pairwise coprime; and $N^{+} M$ is neat for the Hilbert modular surface associated to $F$ and the Shimura curve associated to an arbitrary indefinite quaternion algebra $B_{N^{\sim}}$ with $N^{\sim}$ coprime to $N^{+} M$;

(R2): $\rho_{\sigma}$ is residually surjective. In other words, for a stable lattice $\mathrm{T}_{\sigma}$ of $\mathrm{V}_{\sigma}, \bar{\rho}_{\sigma}$ is surjective (which also implies that $\mathrm{T}_{\sigma}$ is unique up to homothety);

(R3): $\bar{\rho}_{\sigma}$ is ramified at all primes $\ell \mid N^{-}$such that $p \mid \ell^{2}-1$;

(R4): for both $\epsilon=+,-$, there exists one strongly $(1, \epsilon)$-admissible prime (see Definition 3.31 and Remark 3.32);

(R5): $p \geq 11$;

(R6): either (and at most one) of the following two holds 
(a): $\rho_{\sigma, \pi}$ is absolutely irreducible and its image contains a non-trivial scalar matrix of finite order coprime to $p$; or

(b): $\pi$ is Asai-decomposable, such that $\rho_{\sigma \times \breve{\pi}}$ is absolutely irreducible and its image contains a non-trivial scalar element of finite order coprime to $p$.

In what follows, we will regard $\sigma$ as its Jacquet-Langlands correspondence to $B_{N^{-}}^{\times}$; in particular, it is an automorphic representation of $B_{N^{-}}^{\times}(\mathbb{A})$ defined over $\mathbb{Q}$.

\subsubsection{A lemma on semisimplicity.}

Lemma 3.9. The $p$-adic representation $\mathrm{H}_{\pi}^{2}\left(X_{\mathfrak{M} ; \mathbb{Q} \text { ac }}\right)$ of $\Gamma_{\mathbb{Q}}$ is isomorphic to ${ }^{\sharp} \mathrm{V}_{\pi}$. In particular, the representation $\mathrm{V}_{\sigma, \pi}$ is tamely pure by Lemma 3.5.

In fact, one can prove the last assertion without using Lemma 3.5.

Proof. By [BL84], the semisimplification of $\mathrm{H}_{\pi}^{2}\left(X_{\mathfrak{M} \text {; } \mathbb{Q}^{\text {ac }}}\right)$ is isomorphic to ${ }^{\sharp} \mathrm{V}_{\pi}$. If $\pi$ is not Asai-decomposable, then ${ }^{\sharp} \mathrm{V}_{\pi}$ is irreducible since $\pi$ is not dihedral and we are done. If $\pi$ is Asai-decomposable, then $\mathrm{H}_{\pi}^{2}\left(X_{\mathfrak{M} ; \mathbb{Q}^{a c}}\right)$ is semisimple. In fact, the Poincaré

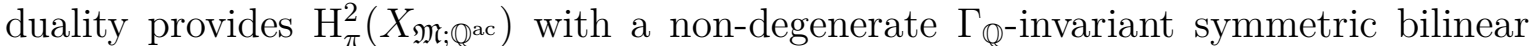
pairing. If $\mathrm{H}_{\pi}^{2}\left(X_{\mathfrak{M} ; \mathbb{Q}^{\text {ac }}}\right)$ is not semisimple, then there will be at least three non-zero direct summands of its semisimplification, which contradicts the fact that ${ }^{\sharp} \mathrm{V}_{\pi}$ has exactly two irreducible direct summands since $\pi$ is non-dihedral.

Notation 3.10. By Lemma 3.9, we will identify $\mathrm{H}_{\pi}^{2}\left(X_{\mathfrak{M} ; \mathbb{Q}^{\mathrm{ac}}}\right)$ with ${ }^{\sharp} \mathrm{V}_{\pi}$ once and for all. Then by abuse of notation,

$$
{ }^{\sharp} \mathrm{T}_{\pi}:=\mathrm{H}_{\pi}^{2}\left(X_{\mathfrak{M} ; \mathbb{Q}^{\mathrm{ac}}}\right) \cap \mathrm{H}_{\text {ét }}^{2}\left(X_{\mathfrak{M} ; \mathbb{Q}^{\mathrm{ac}}}, \mathbb{Z}_{p}(1)\right)
$$

is a stable lattice of ${ }^{\sharp} \mathrm{V}_{\pi}$. The Poincaré duality provides us with a $\Gamma_{\mathbb{Q}}$-invariant perfect pairing

$$
{ }^{\sharp} \overline{\mathrm{T}}_{\pi}^{n} \times{ }^{\sharp} \overline{\mathrm{T}}_{\pi}^{n} \rightarrow \mathbb{Z} / p^{n}
$$

for every integer $n \geq 1$. Put $\mathrm{T}_{\sigma, \pi}=\mathrm{T}_{\sigma} \otimes_{\mathbb{Z}_{p}}{ }^{\sharp} \mathrm{T}_{\pi}$, which is a stable lattice of $\mathrm{V}_{\sigma, \pi}$. Then we have a $\Gamma_{\mathbb{Q}^{-}}$-invariant perfect pairing

$$
\overline{\mathrm{T}}_{\sigma, \pi}^{n} \times \overline{\mathrm{T}}_{\sigma, \pi}^{n} \rightarrow \mathbb{Z} / p^{n}(1)
$$

for every integer $n \geq 1$.

\subsection{Hirzebruch-Zagier classes.}

3.3.1. Cycle class map. Let $X$ be a regular noetherian scheme on which $p$ is invertible. Let $\Lambda$ be either $\mathbb{Q}_{p}$ or $\mathbb{Z} / p^{n}$ for $1 \leq n \leq \infty$. We will assume that suitable adic formalism has been adopted without specification (for our purpose, [Jan88] suffices). Let us first recall the following important fact from algebraic geometry.

Lemma 3.11. Let $X$ be a regular noetherian scheme and $j: Y \rightarrow X$ be a closed immersion of codimension $\geq c$. We have that

(1) $\mathrm{R}^{q} j^{!} \Lambda=0$ for $q<2 c$;

(2) $j^{!} \Lambda \simeq \Lambda[-c](-c)$ if $Y$ is also regular and $j$ is purely of codimension $c$. 
Part (2) is known as the absolute cohomological purity; and (1) is known as the semi-purity which is a consequence of (2). They are proved by Gabber; see [Fuj02].

Notation 3.12. For $c \geq 0$, we denote by $\mathrm{Z}^{c}(X)=\bigoplus_{x} \mathbb{Z} \overline{\{x\}}$ for the abelian group generated by the Zariski closure $\overline{\{x\}} \subset X$ of all (scheme-theoretical) points $x$ of codimension $c$. We equip $\overline{\{x\}}$ with the induced reduced scheme structure.

The same process after [Mil80, Lemma 9.1], which will actually be recalled in the proof of Lemma 4.12, assigns $Z$ a class $\mathrm{cl}_{X}(Z)$ in the étale cohomology $\mathrm{H}_{\text {ét }}^{2 c}(X, \Lambda(c))$. By linearity, we extend this to a map

$$
\mathrm{cl}_{X}: \mathrm{Z}^{c}(X) \rightarrow \mathrm{H}_{\text {ét }}^{2 c}(X, \Lambda(c))
$$

known as the (absolute étale) cycle class map.

If $X$ is smooth over a field, then $\mathrm{cl}_{X}$ factorizes through the group of Chow cycles $\mathrm{CH}^{c}(X)$. Moreover, the map $\mathrm{cl}_{X}$ is multiplicative with respect to the intersection product on Chow groups and the cup product on étale cohomology groups.

3.3.2. General étale Abel-Jacobi map. Let $k$ be a field of characteristic not $p$, and $Z$ a smooth proper scheme over $k$. For each integer $e$, we have the Hochschild-Serre spectral sequence $\mathrm{E}^{p, q}$ converging to $\mathrm{H}_{\text {ét }}^{p+q}\left(Z_{k^{\text {ac }}}, \Lambda(e)\right)$ with

$$
\mathrm{E}_{2}^{p, q}=\mathrm{H}^{p}\left(k, \mathrm{H}_{\text {ét }}^{q}\left(Z_{k^{\text {ac }}}, \Lambda(e)\right)\right) .
$$

By restriction, we have the map

$$
\xi_{Z}^{0}: \mathrm{H}_{\text {ét }}^{q}(Z, \Lambda(e)) \rightarrow \mathrm{H}^{0}\left(k, \mathrm{H}_{\text {ét }}^{q}\left(Z_{k^{\mathrm{ac}}}, \Lambda(e)\right)\right) .
$$

Denote by $\mathrm{H}_{\text {ét }}^{q}(Z, \Lambda(e))^{0}$ the kernel of $\xi_{Z}^{0}$. The spectral sequence yields an edge map

$$
\xi_{Z}^{1}: \mathrm{H}_{\text {ét }}^{q}(Z, \Lambda(e))^{0} \rightarrow \mathrm{H}^{1}\left(k, \mathrm{H}_{\text {ét }}^{q-1}\left(Z_{k^{\mathrm{ac}}}, \Lambda(e)\right)\right) .
$$

For $c \geq 0$, put $\mathrm{CH}^{c}(Z, \Lambda)=\mathrm{CH}^{c}(Z) \otimes_{\mathbb{Z}} \Lambda$ and recall that we have the cycle class map (3.2). Put $\mathrm{cl}_{Z}^{0}=\xi_{Z}^{0} \circ \mathrm{cl}_{Z}$ and let $\mathrm{CH}^{c}(Z, \Lambda)^{0}$ be its kernel. The composition

$$
\mathrm{cl}_{Z}^{1}:=\xi_{Z}^{1} \circ\left(\left.\mathrm{cl}_{Z}\right|_{\mathrm{CH}^{c}(Z, \Lambda)^{0}}\right): \mathrm{CH}^{c}(Z, \Lambda)^{0} \rightarrow \mathrm{H}^{1}\left(k, \mathrm{H}_{\text {ét }}^{2 c-1}\left(Z_{k^{\mathrm{ac}}}, \Lambda(c)\right)\right)
$$

is usually referred as the étale Abel-Jacobi map.

Lemma 3.13. Let $f: Z^{\prime} \rightarrow Z$ be a smooth proper morphism of equidimension $d$. Then the following diagram commutes

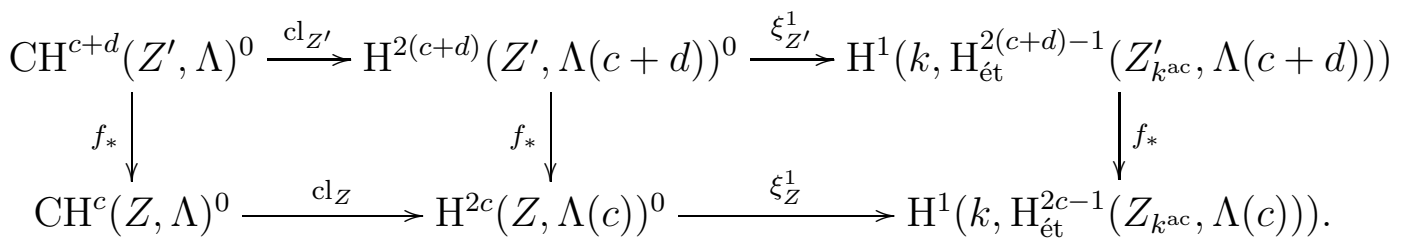

Proof. It follows from the functoriality of cycle class maps (for smooth varieties) and Hochschild-Serre spectral sequences. 
Lemma 3.14. Let notation be as above and suppose that $Z$ is purely of dimension d. Then the following diagram

$$
\begin{aligned}
& \mathrm{CH}^{d}\left(Z \times \times_{\text {Spec } k} Z, \Lambda\right) \times \mathrm{CH}^{c}(Z, \Lambda)^{0} \longrightarrow \mathrm{CH}^{c}(Z, \Lambda)^{0} \\
& \left(\mathrm{cl}_{Z \times \operatorname{Spec} k}^{0}, \mathrm{cl}_{Z}^{1}\right) \downarrow \quad \downarrow \mathrm{cl}_{Z}^{1} \\
& \mathrm{H}_{\text {ét }}^{2 d}\left(\left(Z \times_{\text {Spec } k} Z\right)_{k^{\mathrm{ac}}}, \Lambda(d)\right) \times \mathrm{H}^{1}\left(k, \mathrm{H}^{2 c-1}\left(Z_{k^{\mathrm{ac}}}, \Lambda(c)\right)\right) \longrightarrow \mathrm{H}^{1}\left(k, \mathrm{H}^{2 c-1}\left(Z_{k^{\mathrm{ac}}}, \Lambda(c)\right)\right)
\end{aligned}
$$

commutes, where the upper (resp. lower) arrow is induced by the action of Chow (resp. cohomological) correspondences via pullbacks.

Proof. Let $X$ be a smooth proper scheme over $k$. By the multiplicative structure of Hochschild-Serre spectral sequence induced by cup products, we have the following commutative diagram

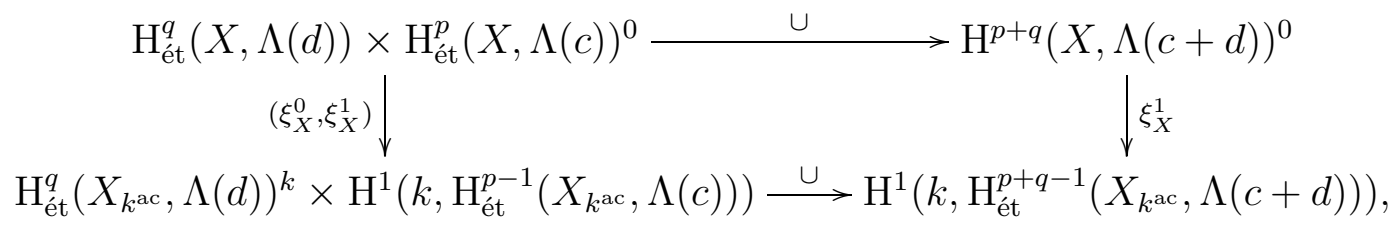

for $p \geq 1, q \geq 0$ and $c, d \in \mathbb{Z}$. Then the lemma follows by the above diagram with $X=Z \times_{\text {Spec } k} Z$ and suitable $p, q, c, d$, Lemma 3.13 and the fact that (3.2) is multiplicative.

3.3.3. Construction of Hirzebruch-Zagier classes. The following discussion is under Assumption $3.8(\mathrm{R} 1)$ and that $\wp\left(N^{-}\right)$is even. We take the coefficient ring $\Lambda$ to be $\mathbb{Q}_{p}$. Recall that we have the Hirzebruch-Zagier morphism

$$
\zeta_{N^{+} M, N^{-}}: Y_{N^{+} M, N^{-}} \rightarrow X_{N^{+} M}
$$

and the Hirzebruch-Zagier cycle $\Delta_{N^{+} M, N^{-}}:=\boldsymbol{\Delta}_{N^{+} M, N^{-} ; \mathbb{Q}}$, a Chow cycle in $\mathrm{CH}^{2}\left(Z_{N^{+} M, N^{-}}\right)$where $Z_{N^{+} M, N^{-}}=Y_{N^{+} M, N^{-}} \times_{\operatorname{Spec} \mathbb{Q}} X_{N^{+} M}$.

Choose a $\sigma$-projector $\mathscr{P}_{\sigma}$ and a $\pi$-projector $\mathscr{P}_{\pi}$ (Definitions 2.11 and 2.20), both of level $N^{+} M$. Put $\mathscr{P}_{\sigma, \pi}=\mathscr{P}_{\sigma} \times \mathscr{P}_{\pi}$ as an element in $\operatorname{Corr}\left(Z_{N^{+} M, N^{-}}\right)$. Put

$$
\Delta_{\sigma, \pi}=\mathscr{P}_{\sigma, \pi} \Delta_{N^{+} M, N^{-}} \in \mathrm{CH}^{2}\left(Z_{N^{+} M, N^{-}}\right) \otimes_{\mathbb{Z}} \mathbb{Q},
$$

which in fact belongs to $\mathrm{CH}^{2}\left(Z_{N^{+} M, N^{-}}\right)^{0}$. Recall that we have the corresponding étale Abel--Jacobi map

$$
\mathrm{AJ}_{p}:=\mathrm{cl}_{Z_{N^{+} M, N^{-}}}^{1}: \mathrm{CH}^{2}\left(Z_{N^{+} M, N^{-}}, \mathbb{Q}_{p}\right)^{0} \rightarrow \mathrm{H}^{1}\left(\mathbb{Q}, \mathrm{H}_{\text {ét }}^{3}\left(Z_{N^{+} M, N^{-} ; \mathbb{Q}^{\text {ac }}}, \mathbb{Q}_{p}(2)\right)\right) .
$$

Put

$$
{ }^{p} \Delta_{\sigma, \pi}=\mathrm{AJ}_{p}\left(\Delta_{\sigma, \pi}\right) .
$$

For simplicity, we also put $\mathrm{V}_{\sigma, \pi ; N^{+} M, N^{-}}=\mathrm{H}_{\sigma}^{1}\left(Y_{N^{+} M, N^{-} ; \mathbb{Q}^{\mathrm{ac}}}\right) \otimes_{\mathbb{Q}_{p}} \mathrm{H}_{\pi}^{2}\left(X_{N^{+} M ; \mathbb{Q}^{\mathrm{ac}}}\right)$ as a $p$-adic representation of $\Gamma_{\mathbb{Q}}$. A priori, the Chow cycle $\Delta_{\sigma, \pi}$ depends on the choices of both $\mathscr{P}_{\sigma}$ and $\mathscr{P}_{\pi}$. Nevertheless, we have the following result.

Proposition 3.15. The element ${ }^{p} \Delta_{\sigma, \pi}$ belongs to the subspace $\mathrm{H}_{f}^{1}\left(\mathbb{Q}, \mathrm{V}_{\sigma, \pi ; N^{+} M_{, N^{-}}}\right)$, and is independent of the choices of $\mathscr{P}_{\sigma}$ and $\mathscr{P}_{\pi}$. 
By Lemma 3.9, the $p$-adic representation $\mathrm{V}_{\sigma, \pi ; N^{+} M, N^{-}}$of $\Gamma_{\mathbb{Q}}$ is isomorphic to a nonzero finite sum of copies of $\mathrm{V}_{\sigma, \pi}$. Before the proof, we need a little bit of preparation.

3.3.4. An auxiliary Hecke operator. Choose an auxiliary prime $q \nmid p N M$. Define an operator

$$
\mathscr{T}_{\sigma}^{(q)}=\frac{1}{q+1-\operatorname{tr}\left(\mathrm{Fr}_{q} ; \mathrm{V}_{\sigma}\right)}\left(q+1-T_{N^{+} M, N^{-}}^{(q)}\right)
$$

as an element in $\operatorname{Corr}\left(Z_{N^{+} M, N^{-}}\right)$. This operator was also considered in [BD07, §4.3] and [Zha14, §2.2].

Lemma 3.16. The Chow cycle $\mathscr{T}_{\sigma}^{(q)} \Delta_{N^{+} M, N^{-}}$belongs to $\mathrm{CH}^{2}\left(Z_{N^{+} M, N^{-}}\right)^{0}$.

Proof. By Lemma 2.19 and the Künneth decomposition of algebraic de Rham cohomology, we have

$$
\mathrm{H}_{\mathrm{dR}}^{4}\left(Z_{N^{+} M, N^{-}}\right)=\mathrm{H}_{\mathrm{dR}}^{0}\left(Y_{N^{+} M, N^{-}}\right) \otimes_{\mathbb{Q}} \mathrm{H}_{\mathrm{dR}}^{4}\left(X_{N^{+} M}\right) \oplus \mathrm{H}_{\mathrm{dR}}^{2}\left(Y_{N^{+} M, N^{-}}\right) \otimes_{\mathbb{Q}} \mathrm{H}_{\mathrm{dR}}^{2}\left(X_{N^{+} M}\right) .
$$

The lemma follows since the correspondence $q+1-T_{N^{+}{ }_{M, N^{-}}}^{(q)}$ acts by 0 on both $\mathrm{H}_{\mathrm{dR}}^{0}\left(Y_{N^{+} M, N^{-}}\right)$and $\mathrm{H}_{\mathrm{dR}}^{2}\left(Y_{N^{+} M, N^{-}}\right)$.

Proof of Proposition 3.15. Choose an auxiliary prime $q$ as above. By the definition of $\mathscr{P}_{\sigma, \pi}$ and Lemmas 3.16 and 3.14, we have

$$
\operatorname{AJ}_{p}\left(\mathscr{P}_{\sigma, \pi} \mathscr{T}_{\sigma}^{(q)} \Delta_{N^{+} M, N^{-}}\right)=\mathrm{H}^{1}\left(\mathbb{Q}, \mathscr{P}_{\sigma, \pi}^{\text {ét }}\right) \operatorname{AJ}_{p}\left(\mathscr{T}_{\sigma}^{(q)} \Delta_{N^{+} M, N^{-}}\right)
$$

where

$$
\begin{aligned}
\mathscr{P}_{\sigma, \pi}^{\text {et }}: & \mathrm{H}_{\text {ét }}^{3}\left(Z_{N^{+} M, N^{-} ; \mathbb{Q}^{\text {ac }}}, \mathbb{Q}_{p}(2)\right) \\
& \stackrel{\sim}{\rightarrow} \mathrm{H}_{\text {ét }}^{1}\left(Y_{N^{+} M, N^{-} ; \mathbb{Q}^{\text {ac }}}, \mathbb{Q}_{p}(1)\right) \otimes_{\mathbb{Q}_{p}} \mathrm{H}_{\text {ét }}^{2}\left(X_{N^{+} M ; \mathbb{Q}^{\text {ac }}}, \mathbb{Q}_{p}(1)\right) \\
& \stackrel{\mathscr{P}_{\sigma}^{\text {ét }} \otimes \mathscr{P}_{\pi}^{\text {et }}}{\longrightarrow} \mathrm{H}_{\sigma}^{1}\left(Y_{N^{+} M, N^{-} ; \mathbb{Q}^{\mathrm{ac}}}\right) \otimes_{\mathbb{Q}_{p}} \mathrm{H}_{\pi}^{2}\left(X_{N^{+} M ; \mathbb{Q}^{\mathrm{ac}}}\right)=\mathrm{V}_{\sigma, \pi ; N^{+} M, N^{-}}
\end{aligned}
$$

is the natural projection. On the other hand, we have

$$
\operatorname{AJ}_{p}\left(\mathscr{P}_{\sigma, \pi} \mathscr{T}_{\sigma}^{(q)} \Delta_{N^{+} M, N^{-}}\right)=\operatorname{AJ}_{p}\left(\mathscr{T}_{\sigma}^{(q)} \mathscr{P}_{\sigma, \pi} \Delta_{N^{+} M, N^{-}}\right)
$$

By Lemmas 3.14 and 2.19, and the fact that the induced action of $\mathscr{T}_{\sigma}^{(q)}$ on $\mathrm{H}_{\text {ét }}^{1}\left(Y_{N^{+} M, N^{-} ; \mathbb{Q}^{\text {ac }}}, \mathbb{Q}_{p}(1)\right)$ is invertible, the element $\operatorname{AJ}_{p}\left(\mathscr{P}_{\sigma, \pi} \Delta_{N^{+} M, N^{-}}\right)$itself belongs to $\mathrm{H}^{1}\left(\mathbb{Q}, \mathrm{V}_{\sigma, \pi ; N^{+} M, N^{-}}\right)$. Therefore, we have

$$
\operatorname{AJ}_{p}\left(\mathscr{T}_{\sigma}^{(q)} \mathscr{P}_{\sigma, \pi} \Delta_{N^{+} M, N^{-}}\right)=\operatorname{AJ}_{p}\left(\mathscr{P}_{\sigma, \pi} \Delta_{N^{+} M, N^{-}}\right)
$$

By Lemmas 3.16 and 3.14, the elements $\operatorname{AJ}_{p}\left(\mathscr{P}_{\sigma, \pi} \mathscr{T}_{\sigma}^{(q)} \Delta_{N^{+} M, N^{-}}\right)$and hence $\mathrm{AJ}_{p}\left(\mathscr{P}_{\sigma, \pi} \Delta_{N^{+} M, N^{-}}\right)$are independent of the choices of $\mathscr{P}_{\sigma}$ and $\mathscr{P}_{\pi}$.

Finally, we show that $s:={ }^{p} \Delta_{\sigma, \pi}$ belongs to $\mathrm{H}_{f}^{1}\left(\mathbb{Q}, \mathrm{V}_{\sigma, \pi ; N^{+} M, N^{-}}\right)$. By Lemma 3.9, we only need to check that $\operatorname{loc}_{p}(s) \in \mathrm{H}_{f}^{1}\left(\mathbb{Q}_{p}, \mathrm{~V}_{\sigma, \pi ; N^{+} M, N^{-}}\right)$. By [Nek00, Theorem 3.1 (ii)], $\operatorname{loc}_{p}(s)$ belongs to $\mathrm{H}_{\mathrm{st}}^{1}\left(\mathbb{Q}_{p}, \mathrm{~V}_{\sigma, \pi ; N^{+} M, N^{-}}\right)$. Since the $p$-adic representation $\mathrm{V}_{\sigma, \pi ; N^{+} M, N^{-}}$ of $\Gamma_{p}$ is a direct summand of $\mathrm{H}_{\text {ét }}^{1}\left(Y_{N^{+} M, N^{-} ; \mathbb{Q}_{p}^{\text {ac }}}, \mathbb{Q}_{p}(1)\right) \otimes \mathrm{H}_{\text {cusp }}^{2}\left(X_{N^{+} M ; \mathbb{Q}_{p}^{\text {ac }}}, \mathbb{Q}_{p}(1)\right)$, and for the latter we have $\mathrm{H}_{\mathrm{st}}^{1}\left(\mathbb{Q}_{p},-\right)=\mathrm{H}_{f}^{1}\left(\mathbb{Q}_{p},-\right)$, the proposition follows. 
3.3.5. Statement of main theorem. The following theorem is the main result of the article, in terms of automorphic representations.

Theorem 3.17. Suppose that all of Assumption 3.8 are satisfied.

(1) If $L(1 / 2, \sigma \times \pi) \neq 0$, which in particular implies that $\wp\left(N^{-}\right)$is odd, then

$$
\operatorname{dim}_{\mathbb{Q}_{p}} \mathrm{H}_{f}^{1}\left(\mathbb{Q}, \mathrm{V}_{\sigma, \pi}\right)=0 .
$$

(2) If $\wp\left(N^{-}\right)$is even and ${ }^{p} \Delta_{\sigma, \pi} \neq 0$, then

$$
\operatorname{dim}_{\mathbb{Q}_{p}} \mathrm{H}_{f}^{1}\left(\mathbb{Q}, \mathrm{V}_{\sigma, \pi}\right)=1 .
$$

3.4. Testing factors. From now on until the end of $\$ 5.2$, all discussions will be under Assumption $3.8(\mathrm{R} 1-\mathrm{R} 3)$, and we $f i x$ a prime $q$ not dividing $p N M \operatorname{disc}(F)$, no matter $\wp\left(N^{-}\right)$is even or odd, such that $p \nmid q+1-\operatorname{tr}\left(\operatorname{Fr}_{q} ; \mathrm{V}_{\sigma}\right)$. This is possible due to (R2).

3.4.1. The case where $\wp\left(N^{-}\right)$is even. Suppose that $\wp\left(N^{-}\right)$is even. From now on until the end of $\$ 5.2$, we will assume that ${ }^{p} \Delta_{\sigma, \pi}$ is non-zero in this case.

Recall from Proposition 3.15 that ${ }^{p} \Delta_{\sigma, \pi}$ belongs to $\mathrm{H}_{f}^{1}\left(\mathbb{Q}, \mathrm{V}_{\sigma, \pi ; N^{+} M, N^{-}}\right)$. For each factor $d \mid M$ and $\mathfrak{d} \mid N^{+} M / \mathfrak{M}$, the class

$$
{ }^{p} \Delta_{\sigma, \pi}^{(d, \mathfrak{d})}:=\left(\delta_{N^{+} M, N^{-}}^{(M, d)} \otimes \gamma_{N^{+} M}^{N^{+} M / \mathfrak{M}, \mathfrak{d}}\right)_{*}^{p} \Delta_{\sigma, \pi}
$$

belongs to $\mathrm{H}_{f}^{1}\left(\mathbb{Q}, \mathrm{V}_{\sigma, \pi}\right)$ by Lemma 3.9.

Definition 3.18. A pair of testing factors is a pair $(d, \mathfrak{d})$ as above such that ${ }^{p} \Delta_{\sigma, \pi}^{(d, \mathfrak{d})} \neq 0$.

By the theory of old forms, there exists at least one pair of testing factors and we will fix such a pair $(d, \mathfrak{d})$ once and for all.

3.4.2. The case where $\wp\left(N^{-}\right)$is odd. Suppose that $\wp\left(N^{-}\right)$is odd. From now on until the end of $\$ 5.2$, we will assume that $L(1 / 2, \sigma \times \pi)$ is non-zero in this case.

Since $\mathcal{T}_{N^{+}, N^{-}}$is (non-canonically) isomorphic to the double quotient $B_{N^{-}}^{\times} \backslash\left(B_{N^{-}} \otimes_{\mathbb{Z}}\right.$ $\widehat{\mathbb{Z}})^{\times} /\left(R \otimes_{\mathbb{Z}} \widehat{\mathbb{Z}}\right)^{\times}$for some $R \in \mathcal{T}_{N^{+}, N^{-}}$, the newform (fixed by $\left.\left(R \otimes_{\mathbb{Z}} \widehat{\mathbb{Z}}\right)^{\times}\right)$of the automorphic representation $\sigma$ gives rise to a map

$$
g_{\sigma}: \mathcal{T}_{N^{+}, N^{-}} \rightarrow \mathbb{Z}
$$

whose image is not contained in a proper ideal of $\mathbb{Z}$. Up to \pm 1 , the map $g_{\sigma}$ is independent of the choice of $R$. Similarly, since $\mathcal{S}_{\mathfrak{M}}$ is (non-canonically) isomorphic to the double quotient $Q^{\times} \backslash\left(Q \otimes_{\mathbb{Z}} \widehat{\mathbb{Z}}\right)^{\times} /\left(S \otimes_{\mathbb{Z}} \widehat{\mathbb{Z}}\right)^{\times}$for some $S \in \mathcal{S}_{\mathfrak{M}}$, the newform (fixed by $\left.\left(S \otimes_{\mathbb{Z}} \widehat{\mathbb{Z}}\right)^{\times}\right)$of the automorphic representation $\pi^{Q}$, the Jacquet-Langlands correspondence of $\pi$ to $Q$, gives rise to a surjective map

$$
f_{\pi}: \mathcal{S}_{\mathfrak{M}} \rightarrow \mathbb{Z}
$$

whose image is not contained in a proper ideal of $\mathbb{Z}$. Up to \pm 1 , the map $f_{\pi}$ is independent of the choice of $S$.

Definition 3.19. A pair of testing factors is a pair $(d, \mathfrak{d})$ with $d \mid M$ and $\mathfrak{d} \mid N^{+} M / \mathfrak{M}$ such that

$$
\sum_{t \in \mathcal{T}_{N^{+} M, N^{-}}}\left(\left(\zeta_{N^{+} M, N^{-}}\right)^{*}\left(\gamma_{N^{+} M}^{\left(N^{+} M / \mathfrak{M}, \mathfrak{d}\right)}\right)^{*} f_{\pi}\right)(t) \cdot\left(\left(\delta_{N^{+} M, N^{-}}^{(M, d)}\right)^{*} g_{\sigma}\right)(t) \neq 0 .
$$


Proposition 3.20. There exists a pair of testing factors.

In what follows, we will fix such a pair $(d, \mathfrak{d})$ once and for all. Moreover, put

$$
f_{\pi}^{(\mathfrak{d})}=\left(\gamma_{N^{+} M}^{\left(N^{+} M / \mathfrak{M}, \mathfrak{d}\right)}\right)^{*} f_{\pi}
$$

for simplicity.

Proof. By [Ich08, Theorem 1.1], we have

$$
(3.4)=C \cdot L^{S}(1 / 2, \sigma \times \pi) \cdot \prod_{v \in S} \alpha_{v}(d, \mathfrak{d})
$$

where $S$ is the set of places of $\mathbb{Q}$ consisting of $\infty$ and primes dividing $N M \operatorname{disc}(F)$, $C$ is a non-zero number, and $\alpha_{v}(d, \mathfrak{d})$ is a certain integral of local matrix coefficients depending on $(d, \mathfrak{d})$. We claim that there is a pair $(d, \mathfrak{d})$ such that $\prod_{v \in S} \alpha_{v}(d, \mathfrak{d}) \neq 0$. For $v \nmid N^{+}$, it follows from the lemma below. For $v \mid N^{+}$, the situation is in fact the same as in the lemma below for $v \nmid N$ (one needs to change the role of $\sigma_{v}$ by a component of $\pi_{v}$ as $v$ splits in $F$ and $\pi_{v}$ is unramified).

On the other hand, for each $v \in S$, the local $L$-function $L_{v}(s, \sigma \times \pi)$ does not have a pole at $s=1 / 2$ since $\mathrm{V}_{\sigma, \pi}$ is tamely pure by Lemma 3.9. Therefore, $L^{S}(1 / 2, \sigma \times \pi) \neq 0$, and hence the lemma follows.

Lemma 3.21. Let $v$ be a prime in $S$ that does not divide $N^{+}$. Suppose that $l$ spans the 1-dimensional space $\operatorname{Hom}_{B_{N^{-}}\left(\mathbb{Q}_{v}\right)}\left(\pi_{v}, \sigma_{v}\right)$. Then the image of a new vector of $\pi_{v}$ under $l$ is non-zero.

The dimension of $\operatorname{Hom}_{B_{N^{-}}\left(\mathbb{Q}_{v}\right)}\left(\pi_{v}, \sigma_{v}\right)$ is 1 , and $l \otimes l$ is equal to the matrix coefficient integral $\alpha_{v}$ up to a non-zero constant.

Proof. Denote by $B \subset \mathrm{GL}_{2}$ the standard Borel subgroup of upper-triangular matrices and $T \subset B$ the diagonal subgroup of $\mathrm{GL}_{2}$.

Case 1: $v \nmid N$. Then $B_{N^{-}}$is unramified at $v$. Suppose that $\sigma_{v}$ is the non-normalized induction from $B\left(\mathbb{Q}_{v}\right)$ to $\mathrm{GL}_{2}\left(\mathbb{Q}_{v}\right)$ of an unramified character $\chi$ of $T\left(\mathbb{Q}_{v}\right)$. By Frobenius reciprocity, we have $\operatorname{Hom}_{\mathrm{GL}_{2}\left(\mathbb{Q}_{v}\right)}\left(\pi_{v}, \sigma_{v}\right)=\operatorname{Hom}_{B\left(\mathbb{Q}_{v}\right)}\left(\left.\pi_{v}\right|_{B\left(\mathbb{Q}_{v}\right)}, \chi\right)$.

Choose a non-zero element $j \in F_{v}$ such that $j^{\theta}=-j$. Choose an additive character $\psi$ of $\mathbb{Q}_{v}$ of conductor 0 . We realize $\pi_{v}$ in the Whittaker model with respect to the unipotent radical of $B\left(F_{v}\right)$ and the character $\psi \circ\left(\frac{1}{2} \operatorname{Tr}_{F_{v} / \mathbb{Q}_{v}}\right)$. The following integral

$$
\int_{\mathbb{Q}_{v}^{\times}} W\left(\left(\begin{array}{ll}
j a & \\
& 1
\end{array}\right)\right) \chi\left(\left(\begin{array}{ll}
a & \\
& 1
\end{array}\right)\right) \mathrm{d} a
$$

is absolutely convergent for all functions $W$ in (the above Whittaker model of) $\pi_{v}$, where $\mathrm{d} a$ is a non-zero Haar measure on $\mathbb{Q}_{v}^{\times}$. Moveover, it defines an element in the space $\operatorname{Hom}_{B\left(\mathbb{Q}_{v}\right)}\left(\left.\pi_{v}\right|_{B\left(\mathbb{Q}_{v}\right)}, \chi\right)$. It is easy to calculate that the integral (3.5) is non-zero for a new vector $f$ as $\chi$ is unramified. The lemma follows.

Case 2: $v \mid N^{-}$. Then $B_{N^{-}}$is ramified at $v$ and $v$ is inert in $F$. Thus $\sigma_{v}$ is an unramified character of $D_{v}^{\times}$of order at most 2 , where $D_{v}$ is the division quaternion algebra over $\mathbb{Q}_{v}$. Since $\pi_{v}$ is now unramified, we assume $\pi_{v}=\operatorname{Ind}_{B\left(F_{v}\right)}^{\mathrm{GL}_{2}\left(F_{v}\right)} \chi$ where the induction is normalized. Then the character $\chi^{\prime}$ of $F_{v}^{\times}$defined in [Pra92, §4.1] is trivial. 
Thus, we have $\left.\pi_{v}\right|_{D_{v}^{\times}} \simeq \operatorname{ind}_{F_{v}^{\times}}^{D_{v}^{\times}} \mathbf{1}$. Here, we have fixed a decomposition $D_{v}=F_{v} \oplus F_{v} \Pi$ where $\Pi$ is a uniformizer of $D_{v}$. As $\operatorname{Hom}_{D_{v}^{\times}}\left(\operatorname{ind}_{F_{v}^{\times}}^{D_{x}^{\times}} \mathbf{1}, \sigma_{v}\right) \simeq \operatorname{Hom}_{F_{v}^{\times}}\left(\mathbf{1}, \sigma_{v}\right) \neq\{0\}$, the lemma is equivalent to that

$$
\int_{F_{v}^{\times} \backslash D_{v}^{\times}} \sigma_{v}\left(g^{-1}\right) f(g) \mathrm{d} g \neq 0
$$

for a new vector $f \in \pi_{v}$, where $\mathrm{d} g$ is a Haar measure on $F_{v}^{\times} \backslash D_{v}^{\times}$. The above nonvanishing holds since $f(\Pi) / f(1) \notin\{ \pm 1\}$.

Remark 3.22. In fact, it is not clear whether for all places $v$, the two local $L$-functions $L_{v}(s, \sigma \times \pi)$ defined from the Galois side and $L_{v}^{\mathrm{PSR}}(s, \sigma \times \pi)$ defined by PiatetskiShapiro-Rallis [PSR87] (and used in [Ich08]) coincide or not. Nevertheless, we have

$$
\operatorname{ord}_{s=1 / 2} L(s, \sigma \times \pi)=\operatorname{ord}_{s=1 / 2} L^{\mathrm{PSR}}(s, \sigma \times \pi) .
$$

3.5. Raising levels. In this section, we study only the representation $\sigma$. We collect some results from [BD05] which generalizes the previous work of Ribet [Rib90].

Definition 3.23 (Group A, compare with [BD05, §2.2]). Let $n \geq 1$ be an integer. We say that a prime $\ell$ is $n$-admissible (with respect to $\sigma$ ) if

(A1): $\ell \nmid 2 p q N^{-} \mathcal{D}_{N^{+} M}$;

(A2): $p \nmid \ell^{2}-1$;

(A3): $p^{n} \mid \ell+1-\epsilon_{\sigma}(\ell) \operatorname{tr}\left(\mathrm{Fr}_{\ell} ; \mathrm{V}_{\sigma}\right)$ for some $\epsilon_{\sigma}(\ell) \in\{ \pm 1\}$;

(A4): $p^{n} \mid \ell^{p-1}-1$;

(A5): $\ell$ is inert in $F$.

Remark 3.24. For results in this section, (A4) and (A5) are not used and we actually only need $\ell \nmid p N$ in $(\mathrm{A} 1)$.

Lemma 3.25. Let $\ell$ be an $n$-admissible prime. Then

(1) the submodule $\overline{\mathrm{T}}_{\sigma}^{n}\left[\ell^{2} \mid 1\right]$ is free of rank 1 ;

(2) the natural map $\mathrm{H}^{1}\left(\mathbb{Q}_{\ell^{2}}, \overline{\mathrm{T}}_{\sigma}^{n}\left[\ell^{2} \mid 1\right]\right) \rightarrow \mathrm{H}^{1}\left(\mathbb{Q}_{\ell^{2}}, \overline{\mathrm{T}}_{\sigma}^{n}\right)$ is injective and its image coincides with $\mathrm{H}_{\mathrm{unr}}^{1}\left(\mathbb{Q}_{\ell^{2}}, \overline{\mathrm{T}}_{\sigma}^{n}\right)$;

(3) the induced map $\overline{\mathrm{T}}_{\sigma}^{n}\left[\ell^{2} \mid \ell^{2}\right] \rightarrow \overline{\mathrm{T}}_{\sigma}^{n} / \overline{\mathrm{T}}_{\sigma}^{n}\left[\ell^{2} \mid 1\right]$ is an isomorphism;

(4) both $\mathrm{H}^{1}\left(\mathbb{Q}_{\ell^{2}}, \overline{\mathrm{T}}_{\sigma}^{n}\left[\ell^{2} \mid 1\right]\right)$ and $\mathrm{H}^{1}\left(\mathbb{Q}_{\ell^{2}}, \overline{\mathrm{T}}_{\sigma}^{n}\left[\ell^{2} \mid \ell^{2}\right]\right)$ are isomorphic to $\mathbb{Z} / p^{n}$.

Here we recall from $\$ 1.6 .3$ that $\overline{\mathrm{T}}_{\sigma}^{n}\left[\ell^{2} \mid r\right]$ is the module of $\overline{\mathrm{T}}_{\sigma}^{n}$ on which $\mathrm{I}_{\ell^{2}}$ acts trivially and $\mathrm{Fr}_{\ell^{2}}$ acts by multiplication by $r$.

Proof. These are proved in [GP12, Lemma 8].

By definition, we have an exact sequence

$$
0 \rightarrow \mathrm{H}_{\mathrm{unr}}^{1}\left(\mathbb{Q}_{\ell^{2}}, \overline{\mathrm{T}}_{\sigma}^{n}\right) \rightarrow \mathrm{H}^{1}\left(\mathbb{Q}_{\ell^{2}}, \overline{\mathrm{T}}_{\sigma}^{n}\right) \stackrel{\partial_{\ell^{2}}}{\longrightarrow} \mathrm{H}_{\text {sing }}^{1}\left(\mathbb{Q}_{\ell^{2}}, \overline{\mathrm{T}}_{\sigma}^{n}\right) \rightarrow 0
$$

in which we may fix isomorphisms

$$
\mathrm{u}_{\ell}: \mathrm{H}_{\mathrm{unr}}^{1}\left(\mathbb{Q}_{\ell^{2}}, \overline{\mathrm{T}}_{\sigma}^{n}\right) \stackrel{\sim}{\rightarrow} \mathbb{Z} / p^{n}, \quad \mathbf{s}_{\ell}: \mathrm{H}_{\text {sing }}^{1}\left(\mathbb{Q}_{\ell^{2}}, \overline{\mathrm{T}}_{\sigma}^{n}\right) \stackrel{\sim}{\rightarrow} \mathbb{Z} / p^{n},
$$

by the previous lemma. Only in this section, we use full Hecke algebras as defined in [BD05]. There are two cases. 
3.5.1. The case $\wp\left(N^{-}\right)$is odd. For $1 \leq n \leq \infty$, denote by

$$
g_{\sigma}^{n}: \mathcal{T}_{N^{+}, N^{-}} \rightarrow \mathbb{Z} / p^{n}
$$

the composition of $g_{\sigma}$ (3.3) with the natural map $\mathbb{Z} \rightarrow \mathbb{Z} / p^{n}$. Now we fix an integer $n \geq 1$ and let $\ell$ be an $n$-admissible prime. Applying the argument of [BD05, §5.6] to $g_{\sigma}^{n}$, we obtain a surjective homomorphism

$$
\varphi_{\sigma \mid \ell}^{n}: \mathbb{T}_{N^{+}, N^{-} \ell} \rightarrow \mathbb{Z} / p^{n}
$$

by [BD05, Theorem 5.15], whose kernel is denoted by $\mathcal{J}_{\sigma \mid \ell}^{n}$. Note that condition (2) of [BD05, Theorem 5.15] is satisfied by Assumption 3.8 (R2, R3) and [PW11, Theorem 6.2]. In fact, the condition that $N$ is square-free in [PW11] is unnecessary.

Proposition 3.26. There is a unique isomorphism

$$
\beta: \Phi_{N^{+}, N^{-} \ell}^{(\ell)} / \mathcal{J}_{\sigma \mid \ell}^{n} \stackrel{\sim}{\rightarrow} \mathbb{Z} / p^{n}
$$

rendering the following diagram commutative

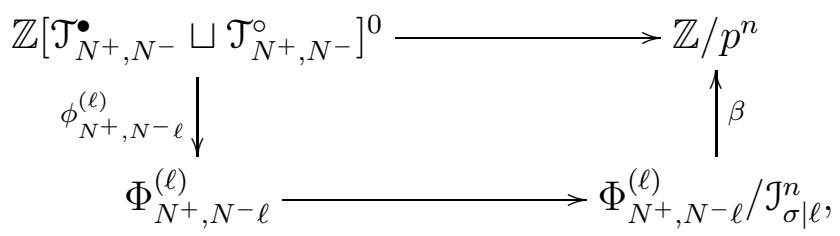

where the upper arrow is induced by the function

$$
g_{\sigma}^{n} \sqcup \epsilon_{\sigma}(\ell) g_{\sigma}^{n}: \mathcal{T}_{N^{+}, N^{-}} \sqcup \mathcal{T}_{N^{+}, N^{-}}^{\circ} \simeq \mathcal{T}_{N^{+}, N^{-}} \sqcup \mathcal{T}_{N^{+}, N^{-}} \rightarrow \mathbb{Z} / p^{n} ;
$$

the group $\Phi_{N^{+}, N^{-} \ell}^{(\ell)}$ is defined in Notation 2.10; and the map $\phi_{N^{+}, N^{-\ell}}^{(\ell)}$ is the one in [BD05, Corollary 5.12].

Proof. It follows from the proof of [BD05, Theorem 5.15].

Proposition 3.27. There is unique isomorphism

$$
\hat{\beta}: \mathrm{T}_{p}\left(J_{N^{+}, N^{-} \ell}\right) / \mathrm{J}_{\sigma \mid \ell}^{n} \rightarrow \overline{\mathrm{T}}_{\sigma}^{n}
$$

of $\mathbb{Z} / p^{n}\left[\Gamma_{\mathbb{Q}}\right]$-modules rendering the following diagram commutative

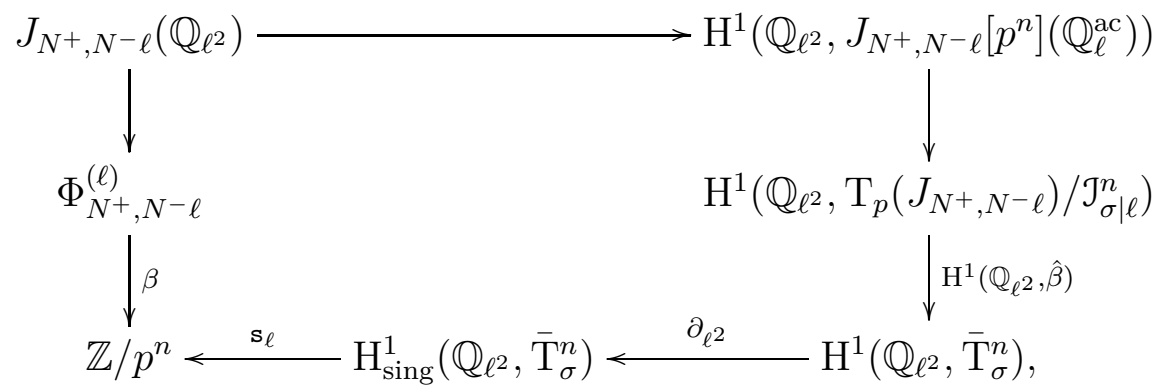

where the upper horizontal arrow is the Kummer map; and the first left vertical arrow is the reduction map.

Proof. It follows from the proof of [BD05, Theorem 5.17, Corollary 5.18]. 
Note that there is a canonical $\Gamma_{\mathbb{Q}}$-equivariant $\mathbb{Z}_{p}$-linear isomorphism between the Tate module $\mathrm{T}_{p}\left(J_{N^{+}, N^{-} \ell}\right)$ and $\mathrm{H}_{\text {ét }}^{1}\left(Y_{N^{+}, N^{-} \ell}, \mathbb{Z}_{p}(1)\right)$. Therefore, Proposition 3.29 yields a projection

$$
\psi_{\ell}^{n}: \mathrm{H}_{\text {ét }}^{1}\left(Y_{N^{+}, N^{-} \ell ; \mathbb{Q}^{\text {ac }}}, \mathbb{Z} / p^{n}(1)\right) \rightarrow \overline{\mathrm{T}}_{\sigma}^{n} .
$$

3.5.2. The case $\wp\left(N^{-}\right)$is even. The automorphic representation $\sigma$ gives rise to a surjective homomorphism $\varphi_{\sigma}: \mathbb{T}_{N^{+}, N^{-}} \rightarrow \mathbb{Z}_{p}$. For $1 \leq n \leq \infty$, denote by $\varphi_{\sigma}^{n}$ the composition of $\varphi_{\sigma}$ with the natural map $\mathbb{Z}_{p} \rightarrow \mathbb{Z} / p^{n}$, and $\mathcal{J}_{\sigma}^{n}$ the kernel of $\varphi_{\sigma}^{n}$.

Now we fix an integer $n \geq 1$. Assumption 3.8 (R2, R3) imply that $\mathrm{T}_{p}\left(J_{N^{+}, N^{-}}\right) / \mathcal{J}_{\sigma}^{n}$ is isomorphic to $\overline{\mathrm{T}}_{\sigma}^{n}$ as a $\mathbb{Z} / p^{n}\left[\Gamma_{\mathbb{Q}}\right]$-module, by [Wil95, Theorem 2.1] and [Hel07, Corollary 8.11, Remark 8.12]. Take an $n$-admissible prime $\ell_{1}$. The Kummer map

$$
J_{N^{+}, N^{-}}\left(\mathbb{Q}_{\ell_{1}^{2}}\right) / \mathcal{J}_{\sigma}^{n} \rightarrow \mathrm{H}^{1}\left(\mathbb{Q}_{\ell_{1}^{2}}, \overline{\mathrm{T}}_{p}^{\infty}\left(J_{N^{+}, N^{-}}\right) / \mathcal{J}_{\sigma}^{n}\right)
$$

induces a map

$$
J_{N^{+}, N^{-}}\left(\mathbb{Q}_{\ell_{1}^{2}}\right) / \mathcal{J}_{\sigma}^{n} \rightarrow \mathrm{H}^{1}\left(\mathbb{Q}_{\ell_{1}^{2}}, \overline{\mathrm{T}}_{\sigma}^{n}\right),
$$

whose image is contained in $\mathrm{H}_{\mathrm{unr}}^{1}\left(\mathbb{Q}_{\ell_{1}^{2}}, \overline{\mathrm{T}}_{\sigma}^{n}\right)$. Running the same argument on [BD05, Page 57], we obtain a natural surjective map

$$
\mathbb{Z}\left[\mathcal{T}_{N^{+}, N^{-} \ell_{1}}\right] \rightarrow \mathrm{H}_{\mathrm{unr}}^{1}\left(\mathbb{Q}_{\ell_{1}^{2}}, \overline{\mathrm{T}}_{\sigma}^{n}\right) \stackrel{\mathrm{u}_{\ell_{1}}}{\longrightarrow} \mathbb{Z} / p^{n} .
$$

It induces a function

$$
g_{\sigma \mid \ell_{1}}^{n}: \mathcal{T}_{N^{+}, N^{-} \ell_{1}} \rightarrow \mathbb{Z} / p^{n}
$$

which satisfies $g_{\sigma \mid \ell_{1}}^{n} \circ \mathrm{op}_{\ell_{1}}=\epsilon_{\sigma}\left(\ell_{1}\right) g_{\sigma \mid \ell_{1}}^{n}$.

Let $\ell_{2}$ be an $n$-admissible prime other than $\ell_{1}$. Applying the argument of [BD05, §5.6] to $g_{\sigma \mid \ell_{1}}^{n}$ with $N^{+}=N^{+}, N^{-}=N^{-} \ell_{1}, m=\ell_{1}$ and $\ell=\ell_{2}$, we obtain a surjective homomorphism

$$
\varphi_{\sigma \mid \ell_{1}, \ell_{2}}^{n}: \mathbb{T}_{N^{+}, N^{-} \ell_{1} \ell_{2}} \rightarrow \mathbb{Z} / p^{n}
$$

by $\left[\mathrm{BD} 05\right.$, Theorem 5.15]. We denote its kernel by $\mathcal{J}_{\sigma \mid \ell_{1}, \ell_{2}}^{n}$. We have the following results similar to Propositions 3.26 and 3.27.

Proposition 3.28. There is a unique isomorphism

$$
\beta: \Phi_{N^{+}, N^{-} \ell_{1} \ell_{2}}^{\left(\ell_{2}\right)} / \mathcal{J}_{\sigma \mid \ell_{1}, \ell_{2}}^{n} \stackrel{\sim}{\rightarrow} \mathbb{Z} / p^{n}
$$

rendering the following diagram commutative

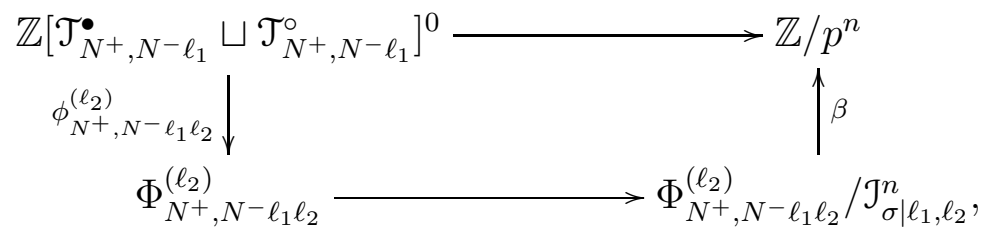

where the upper arrow is induced by the function $g_{\sigma \mid \ell_{1}}^{n} \sqcup \epsilon_{\sigma}\left(\ell_{2}\right) g_{\sigma \mid \ell_{1}}^{n}$. 
Proposition 3.29. There is unique isomorphism

$$
\hat{\beta}: \mathrm{T}_{p}\left(J_{N^{+}, N^{-} \ell_{1} \ell_{2}}\right) / \mathcal{J}_{\sigma \mid \ell_{1}, \ell_{2}}^{n} \rightarrow \overline{\mathrm{T}}_{\sigma}^{n}
$$

of $\mathbb{Z} / p^{n}\left[\Gamma_{\mathbb{Q}}\right]$-modules rendering the following diagram commutative

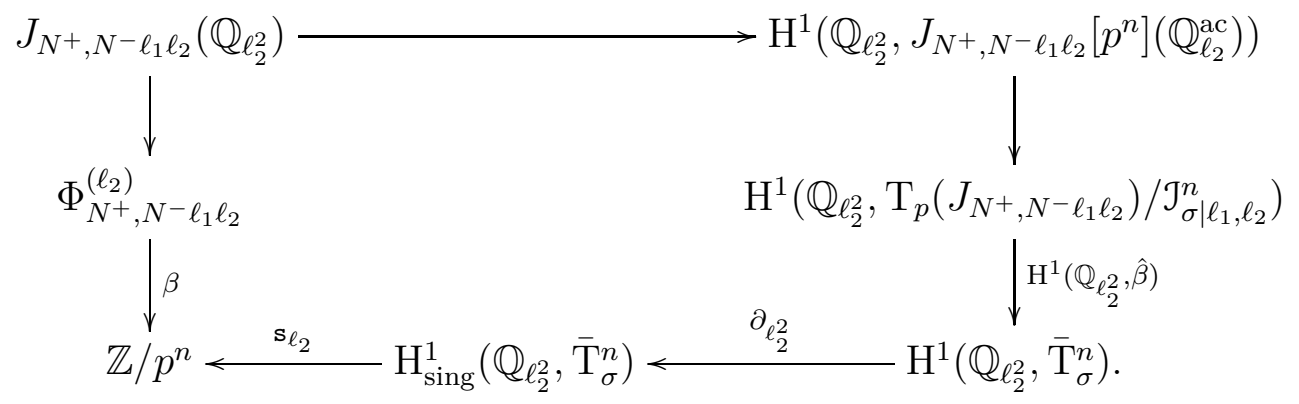

Note that there is a canonical $\Gamma_{\mathbb{Q}^{-}}$equivariant $\mathbb{Z}_{p}$-linear isomorphism between the Tate module $\mathrm{T}_{p}\left(J_{N^{+}, N^{-} \ell_{1} \ell_{2}}\right)$ and $\mathrm{H}_{\text {ét }}^{1}\left(Y_{N^{+}, N^{-} \ell_{1} \ell_{2}}, \mathbb{Z}_{p}(1)\right)$. Therefore, Proposition 3.29 yields a projection

$$
\psi_{\ell_{1}, \ell_{2}}^{n}: \mathrm{H}_{\text {ét }}^{1}\left(Y_{N^{+}, N^{-} \ell_{1} \ell_{2} ; \mathbb{Q}^{\mathrm{ac}}}, \mathbb{Z} / p^{n}(1)\right) \rightarrow \overline{\mathrm{T}}_{\sigma}^{n} .
$$

3.6. Construction of annihilators. In this section, the coefficient ring $\Lambda$ will be $\mathbb{Z} / p^{n}$ for $1 \leq n \leq \infty$. In order to emphasize the exponent $n$, we will use the notation [ $]_{Z}^{n}$ for the étale Abel-Jacobi map $\mathrm{cl}_{Z}^{1}$ with the coefficient ring $\Lambda=\mathbb{Z} / p^{n}$.

Let $N^{\sim}$ be a product of even number of distinct primes that are inert in $F$, such that $N^{-}$divides $N^{\sim}$ and $N^{\sim} / N^{-}$is coprime to $2 p q \mathcal{D}_{N^{+} M}$. In what follows, we will regard the schemes $y_{N^{+} M, N^{\sim}}, x_{N^{+} M}, z_{N^{+} M, N^{\sim}}$ and the Hirzebruch-Zagier morphism $\zeta_{N^{+} M, N^{\sim}}$ as over $\mathbb{Z}\left[1 / q \mathcal{D}_{N^{+} M}\right]$. Recall that we have the cycle $\boldsymbol{\Delta}_{N^{+} M, N^{\sim}}$ which is the graph of $\zeta_{N^{+} M, N^{\sim}}$, as an element in $\mathrm{Z}^{2}\left(z_{N^{+} M, N^{\sim}}\right)$. We also have two degeneracy morphisms

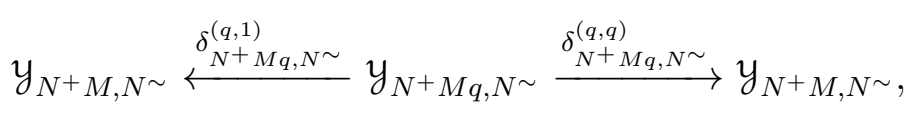

which induce two morphisms

$$
\begin{aligned}
& \zeta_{N^{+} M, N^{\sim}}^{\dagger}:=\zeta_{N^{+} M q, N^{\sim}} \circ \delta_{N^{+} M q, N^{\sim}}^{(q, 1)}: y_{N^{+} M q, N^{\sim}} \rightarrow x_{N^{+} M}, \\
& \zeta_{N^{+} M, N^{\sim}}^{\ddagger}:=\zeta_{N^{+} M q, N^{\sim}} \circ \delta_{N^{+} M q, N^{\sim}}^{(q, q)}: y_{N^{+} M q, N^{\sim}} \rightarrow x_{N^{+} M} .
\end{aligned}
$$

Put $Z_{N^{+} M, N^{\sim}}^{\prime}=y_{N^{+} M q, N \sim} \times_{\operatorname{Spec} \mathbb{Z}\left[1 / \mathcal{D}^{\mathcal{D}^{+}}{ }_{M}\right]} x_{N^{+} M}$. Denote the graphs of $\zeta_{N^{+} M q, N^{\sim}}^{\dagger}$ and $\zeta_{N^{+} M q, N^{\sim}}^{\ddagger}$ by $\boldsymbol{\Delta}_{N^{+} M, N^{\sim}}^{\dagger}$ and $\boldsymbol{\Delta}_{N^{+} M, N^{\sim}}^{\ddagger}$, respectively, which are both smooth over the base $\mathbb{Z}\left[1 / q \mathcal{D}_{N^{+} M}\right]$. We also have the étale projection

$$
\delta^{\prime}:=\delta_{N^{+} M q, N^{\sim}}^{(q, 1)} \times \mathrm{id}: z_{N^{+} M, N^{\sim}}^{\prime} \rightarrow z_{N^{+} M, N^{\sim}}
$$

under which the image of $\boldsymbol{\Delta}_{N^{+} M, N^{\sim}}^{\dagger}$ is simply $\boldsymbol{\Delta}_{N^{+} M, N^{\sim}}$. We remark that the image of $\boldsymbol{\Delta}_{N^{+} M, N^{\sim}}^{\ddagger}$ under $\delta^{\prime}$ is not necessarily smooth over the base. Put

$$
\boldsymbol{\Delta}_{N^{+} M, N^{\sim}}^{\prime}=\frac{1}{q+1-\operatorname{tr}\left(\mathrm{Fr}_{q} ; \mathrm{V}_{\sigma}\right)}\left(\boldsymbol{\Delta}_{N^{+} M, N^{\sim}}^{\dagger}-\boldsymbol{\Delta}_{N^{+} M, N^{\sim}}^{\ddagger}\right)
$$

as a cycle of $z_{N^{+} M, N^{\sim}}^{\prime}$. Put $\Delta_{N^{+} M, N^{\sim}}^{\prime}=\boldsymbol{\Delta}_{N^{+} M, N^{\sim} ; \mathbb{Q}}^{\prime}$ as usual. 
Lemma 3.30. We have that

(1) for every point $\operatorname{Spec} k \rightarrow \operatorname{Spec} \mathbb{Z}\left[1 / p q N^{\sim} \mathcal{D}_{N^{+} M}\right]$ where $k$ is a field and every coefficient ring $\Lambda=\mathbb{Z} / p^{n}$ with arbitrary $1 \leq n \leq \infty$, the (induced) Chow cycle (of) $\boldsymbol{\Delta}_{N^{+} M, N^{\sim} ; k}^{\prime}$ belongs to $\mathrm{CH}^{2}\left(Z_{N^{+} M, N^{\sim} ; k}^{\prime}, \Lambda\right)^{0}$;

(2) $\delta_{\mathbb{Q} *}^{\prime} \Delta_{N^{+} M, N^{\sim}}^{\prime}=\mathscr{T}_{\sigma}^{(q)} \Delta_{N^{+} M, N^{\sim}}$ as an equality in $\mathrm{CH}^{2}\left(Z_{N^{+} M, N^{\sim}}\right) \otimes_{\mathbb{Z}} \mathbb{Q}$;

(3) for arbitrary $1 \leq n \leq \infty$,

$$
\delta_{\mathbb{Q} *}^{\prime}\left[\Delta_{N^{+} M, N^{\sim}}^{\prime}\right]_{\mathcal{Z}_{N^{+} M_{M, N} \sim \mathbb{Q}}^{\prime}}^{n}=\left[\mathscr{T}_{\sigma}^{(q)} \Delta_{N^{+} M, N^{\sim}}\right]_{Z_{N^{+} M, N^{\sim}}}^{n} .
$$

Proof. Part (1) follows from the same proof of Lemma 3.16, and the Comparison Theorem for étale cohomology with $\mathbb{Z}_{p}$-coefficient. Part (2) follows directly from the construction. Part (3) follows from (2) and Lemma 3.13.

3.6.1. The case $N^{\sim}=N^{-}$. Assume that $\wp\left(N^{-}\right)$is even and $N^{\sim}=N^{-}$. Recall that we have fixed a pair of testing factors $(d, \mathfrak{d})$. Consider the following composite map

$$
\begin{aligned}
\mathscr{P}_{\sigma, \pi}^{(d, \mathfrak{d})}: & \mathrm{H}_{\text {ét }}^{3}\left(Z_{N^{+} M q, N^{-} ; \mathbb{Q}^{\mathrm{ac}}}, \mathbb{Z}_{p}(2)\right) \\
& \rightarrow \mathrm{H}_{\text {ét }}^{1}\left(Y_{N^{+} M q, N^{-} ; \mathbb{Q}^{\mathrm{ac}}}, \mathbb{Z}_{p}(1)\right) \otimes_{\mathbb{Z}_{p}} \mathrm{H}_{\text {ét }}^{2}\left(X_{N^{+} M ; \mathbb{Q}^{\mathrm{ac}}}, \mathbb{Z}_{p}(1)\right) \\
& \rightarrow \mathrm{H}_{\text {ét }}^{1}\left(Y_{N^{+}, N^{-} ; \mathbb{Q}^{\mathrm{ac}}}, \mathbb{Z}_{p}(1)\right) \otimes_{\mathbb{Z}_{p}} \mathrm{H}_{\text {ét }}^{2}\left(X_{\mathfrak{M} ; \mathbb{Q}^{\mathrm{ac}}}, \mathbb{Z}_{p}(1)\right) \\
& \rightarrow \mathrm{T}_{\sigma} \otimes_{\mathbb{Z}_{p}}{ }^{\sharp} \mathrm{T}_{\pi}=\mathrm{T}_{\sigma, \pi},
\end{aligned}
$$

where the second last arrow is induced by $\delta_{N^{+} M q, N^{-}}^{(M q,)} \otimes \gamma_{\mathfrak{M}}^{\left(N^{+} M / \mathfrak{M}, \mathfrak{d}\right)}$; and the last one is the projection to the $(\sigma, \pi)$-component.

Introduce the class

$$
\bar{\Delta}_{\sigma, \pi}^{\infty}=\mathrm{H}^{1}\left(\mathbb{Q}, \mathscr{P}_{\sigma, \pi}^{(d, \mathfrak{d})}\right)\left(\left[\Delta_{N^{+} M, N^{-}}^{\prime}\right]_{Z_{N^{+} M q, N^{-}}}^{\infty}\right) \in \mathrm{H}^{1}\left(\mathbb{Q}, \mathrm{T}_{\sigma, \pi}\right) .
$$

Then $\bar{\Delta}_{\sigma, \pi}^{\infty}$ is non-torsion by Definition 3.18, the proof of Proposition 3.15, and Lemma 3.30 (3). It induces classes $\bar{\Delta}_{\sigma, \pi}^{n} \in \mathrm{H}^{1}\left(\mathbb{Q}, \overline{\mathrm{T}}_{\sigma, \pi}^{n}\right)$ for all $1 \leq n \leq \infty$, where we recall that $\overline{\mathrm{T}}_{\sigma, \pi}^{n}=\mathrm{T}_{\sigma, \pi} \otimes_{\mathbb{Z}_{p}} \mathbb{Z} / p^{n}$.

3.6.2. The case $N^{\sim} \neq N^{-}$. We first define strongly admissible primes.

Definition 3.31 (Group $\mathbf{S}$ ). Let $n \geq 1$ be an integer. We say that a prime $\ell$ is strongly $(n, \epsilon)$-admissible (with respect to $\sigma$ and $\pi$ ) if

(S1): $\ell$ is $n$-admissible (Definition 3.23);

(S2): $\epsilon=-\epsilon_{\sigma}(\ell) \breve{\eta}\left(\mathrm{Fr}_{\ell}\right)$, where $\breve{\eta}$ is defined in $\S 3.2 .1$;

(S3): $\operatorname{tr}\left(\mathrm{Fr}_{\ell^{2}} ; \mathrm{V}_{\pi}\right) \bmod p \notin\left\{2 \ell,-2 \ell, \ell^{2}+1,-\ell^{2}-1\right\}$, and

- $\operatorname{tr}\left(\mathrm{Fr}_{\ell^{2}} ; \mathrm{V}_{\pi}\right) \bmod p \neq 0$ if $\mathbb{F}_{p}^{\times}$contains an element of order 4 ;

- $\operatorname{tr}\left(\mathrm{Fr}_{\ell^{2}} ; \mathrm{V}_{\pi}\right)$ mod $p \neq-\ell$ if $\mathbb{F}_{p}^{\times}$contains an element of order 3;

- $\operatorname{tr}\left(\mathrm{Fr}_{\ell^{2}} ; \mathrm{V}_{\pi}\right) \bmod p \neq \ell$ if $\mathbb{F}_{p}^{\times}$contains an element of order 6 .

A prime $\ell$ is strongly $n$-admissible if it is either strongly $(n,+)$-admissible or strongly $(n,-)$-admissible.

Remark 3.32 . 
(1) For $\epsilon= \pm$, if there exists one strongly $(1, \epsilon)$-admissible prime, then the density of strongly $(n, \epsilon)$-admissible primes among all $n$-admissible primes is strictly positive, and is independent of $n$.

(2) Assumption (S3) is equivalent to that the conjugacy class $\left(\operatorname{As} \rho_{\pi}\right)(-1)\left(\mathrm{Fr}_{\ell}\right)$ is semisimple, whose $\bmod p$ reduction contains none of

$$
\left(\begin{array}{llll}
\mu & & & \\
& 1 & & \\
& & -1 & \\
& & & \mu^{-1}
\end{array}\right), \quad\left(\begin{array}{cccc} 
\pm \ell & & & \\
& 1 & & \\
& & -1 & \\
& & & \pm \ell^{-1}
\end{array}\right)
$$

for all $\mu \in \mathbb{F}_{p}^{\times}$of order in $\{1,2,3,4,6\}$.

When $\wp\left(N^{-}\right)$is even, let $\ell_{1}$ and $\ell_{2}$ be two distinct strongly $n$-admissible primes, and put $N^{\sim}=N^{-} \ell_{1} \ell_{2}$. When $\wp\left(N^{-}\right)$is odd, let $\ell$ be a strongly $n$-admissible primes, and put $N^{\sim}=N^{-} \ell$. We have the following composite map

$$
\begin{aligned}
\mathscr{Q}_{\sigma, \pi}^{(d, \mathfrak{d})} & : \mathrm{H}_{\text {ét }}^{3}\left(Z_{N^{+} M q, N^{\sim} ; \mathbb{Q}^{\mathrm{ac}}}, \mathbb{Z}_{p}(2)\right) \\
& \rightarrow \mathrm{H}_{\text {ét }}^{1}\left(Y_{N^{+} M q, N^{\sim} ; \mathbb{Q}^{\mathrm{ac}}}, \mathbb{Z} / p^{n}(1)\right) \otimes_{\mathbb{Z}_{p}} \mathrm{H}_{\text {ét }}^{2}\left(X_{N^{+} M ; \mathbb{Q}^{\mathrm{ac}}}, \mathbb{Z}_{p}(1)\right) \\
& \rightarrow \mathrm{H}_{\text {ét }}^{1}\left(Y_{N^{+}, N^{\sim} ; \mathbb{Q}^{\mathrm{ac}}}, \mathbb{Z} / p^{n}(1)\right) \otimes_{\mathbb{Z}_{p}} \mathrm{H}_{\text {ét }}^{2}\left(X_{\mathfrak{M} ; \mathbb{Q}^{\mathrm{ac}}}, \mathbb{Z}_{p}(1)\right) \\
& \rightarrow \overline{\mathrm{T}}_{\sigma}^{n} \otimes_{\mathbb{Z}_{p}}{ }^{\sharp} \mathrm{T}_{\pi}=\overline{\mathrm{T}}_{\sigma, \pi}^{n},
\end{aligned}
$$

where the second last arrow is induced by $\delta_{N^{+} M q, N^{\sim}}^{(M, d)} \otimes \gamma_{\mathfrak{M}}^{\left(N^{+} M / \mathfrak{M}, \mathfrak{o}\right)}$; and the last one is induced by $\psi_{\ell_{1}, \ell_{2}}^{n}(3.11)$ (resp. $\psi_{\ell}^{n}(3.8)$ ) when $\wp\left(N^{-}\right)$is even (resp. odd). Finally, we denote by $\bar{\Delta}_{\sigma, \pi \mid \ell_{1}, \ell_{2}}^{n}\left(\operatorname{resp} . \bar{\Delta}_{\sigma, \pi \mid \ell}^{n}\right)$ the class

$$
\mathrm{H}^{1}\left(\mathbb{Q}, \mathscr{Q}_{\sigma, \pi}^{(d, \mathfrak{d})}\right)\left(\left[\Delta_{N^{+} M, N^{\sim}}^{\prime}\right]_{Z_{N^{+}}{ }_{M q, N} \sim}^{n}\right) \in \mathrm{H}^{1}\left(\mathbb{Q}, \overline{\mathrm{T}}_{\sigma, \pi}^{n}\right)
$$

when $\wp\left(N^{-}\right)$is even (resp. odd).

\section{Congruences of Hirzebruch-Zagier Classes}

This chapter is the technical heart of the article, in which we propose and prove some explicit congruence formulae for Hirzebruch-Zagier classes. In §4.1, we study the local cohomology of the Galois representation in question at various primes, using local Tate pairings. In $\S 4.2$, we state the result about the integral Tate conjecture for special fibers of Hilbert modular surfaces at good inert primes, in the version convenient for our use. In $\S 4.3$, we state our theorems on explicit congruence formulae for Hirzebruch-Zagier classes. The last two sections are devoted to the proof of previous theorems, in which $\S 4.4$ is responsible for the case of smooth reduction and $\$ 4.5$ is responsible for the case of semistable reduction.

4.1. Local cohomology and Tate duality. We will proceed under Assumption 3.8 $(\mathrm{R} 1-\mathrm{R} 4)$ till the end of this chapter. In this section, we study various localizations of the Galois cohomology $\mathrm{H}^{1}\left(\mathbb{Q}, \overline{\mathrm{T}}_{\sigma, \pi}^{n}\right)$. Let $n \geq 1$ be an integer. 
The $\Gamma_{\mathbb{Q}}$-invariant pairing $\overline{\mathrm{T}}_{\sigma, \pi}^{n} \times \overline{\mathrm{T}}_{\sigma, \pi}^{n} \rightarrow \mathbb{Z} / p^{n}(1)$ (3.1) induces, for each prime power $v$, a local Tate pairing

$$
\langle,\rangle_{v}: \mathrm{H}^{1}\left(\mathbb{Q}_{v}, \overline{\mathrm{T}}_{\sigma, \pi}^{n}\right) \times \mathrm{H}^{1}\left(\mathbb{Q}_{v}, \overline{\mathrm{T}}_{\sigma, \pi}^{n}\right) \rightarrow \mathrm{H}^{2}\left(\mathbb{Q}_{v}, \mathbb{Z} / p^{n}(1)\right) \simeq \mathbb{Z} / p^{n} .
$$

In what follows, we will write $\left\langle s_{1}, s_{2}\right\rangle_{v}$ rather than $\left\langle\operatorname{loc}_{v}\left(s_{1}\right), \operatorname{loc}_{v}\left(s_{2}\right)\right\rangle_{v}$ for $s_{1}, s_{2} \in$ $\mathrm{H}^{1}\left(\mathbb{Q}, \overline{\mathrm{T}}_{\sigma, \pi}^{n}\right)$.

Lemma 4.1. The restriction of $\sum_{v}\langle,\rangle_{v}$ to $\mathrm{H}^{1}\left(\mathbb{Q}, \overline{\mathrm{T}}_{\sigma, \pi}^{n}\right)$ under the map $\prod_{v} \operatorname{loc}_{v}$ is a finite sum and moreover equal to zero, where both the sum and the product are taken over all primes $v$.

Proof. It follows from the global class field theory, and the convention that $p$ is odd.

4.1.1. The case $v \nmid p$.

Proposition 4.2. Suppose that $v=\ell$ is a strongly $n$-admissible prime. Let $\alpha \in\{1,2\}$ be an exponent.

(1) The natural map $\mathrm{H}^{1}\left(\mathbb{Q}_{\ell^{\alpha}}, \overline{\mathrm{T}}_{\sigma}^{n} \otimes_{\mathbb{Z} / p^{n}}{ }^{\sharp} \overline{\mathrm{T}}_{\pi}^{n}\left[\ell^{2} \mid 1\right]\right) \rightarrow \mathrm{H}^{1}\left(\mathbb{Q}_{\ell^{\alpha}}, \overline{\mathrm{T}}_{\sigma, \pi}^{n}\right)$ is an isomorphism.

(2) The $\mathbb{Z} / p^{n}$-module $\mathrm{H}^{1}\left(\mathbb{Q}_{\ell^{\alpha}}, \overline{\mathrm{T}}_{\sigma, \pi}^{n}\right)$ is free of rank $2 \alpha$.

(3) The natural map $\mathrm{H}^{1}\left(\mathbb{Q}_{\ell^{\alpha}}, \overline{\mathrm{T}}_{\sigma}^{n}\left[\ell^{2} \mid 1\right] \otimes_{\mathbb{Z} / p^{n}}{ }^{\sharp} \overline{\mathrm{T}}_{\pi}^{n}\left[\ell^{2} \mid 1\right]\right) \rightarrow \mathrm{H}^{1}\left(\mathbb{Q}_{\ell^{\alpha}}, \overline{\mathrm{T}}_{\sigma, \pi}^{n}\right)$ is injective with the image $\mathrm{H}_{\mathrm{unr}}^{1}\left(\mathbb{Q}_{\ell^{\alpha}}, \overline{\mathrm{T}}_{\sigma, \pi}^{n}\right)$.

(4) The natural map $\mathrm{H}^{1}\left(\mathbb{Q}_{\ell^{\alpha}}, \overline{\mathrm{T}}_{\sigma}^{n}\left[\ell^{2} \mid \ell^{2}\right] \otimes_{\mathbb{Z} / p^{n}}{ }^{\sharp} \overline{\mathrm{T}}_{\pi}^{n}\left[\ell^{2} \mid 1\right]\right) \rightarrow \mathrm{H}_{\text {sing }}^{1}\left(\mathbb{Q}_{\ell^{\alpha}}, \overline{\mathrm{T}}_{\sigma, \pi}^{n}\right)$ is an isomorphism.

(5) The local Tate pairing $\langle,\rangle_{\ell^{\alpha}}$ induces a perfect pairing between $\mathrm{H}_{\mathrm{unr}}^{1}\left(\mathbb{Q}_{\ell^{\alpha}}, \overline{\mathrm{T}}_{\sigma, \pi}^{n}\right)$ and $\mathrm{H}_{\text {sing }}^{1}\left(\mathbb{Q}_{\ell^{\alpha}}, \overline{\mathrm{T}}_{\sigma, \pi}^{n}\right)$.

(6) The restriction map $\mathrm{H}^{1}\left(\mathbb{Q}_{\ell}, \overline{\mathrm{T}}_{\sigma, \pi}^{n}\right) \rightarrow \mathrm{H}^{1}\left(\mathbb{Q}_{\ell^{2}}, \overline{\mathrm{T}}_{\sigma, \pi}^{n}\right)$ is injective.

Proof. By Definition 3.31, in particular that $\operatorname{tr}\left(\mathrm{Fr}_{\ell^{2}} ; \mathrm{V}_{\pi}\right) \bmod p \notin\{2 \ell,-2 \ell\}$, the $\mathbb{Z} / p^{n}$ -

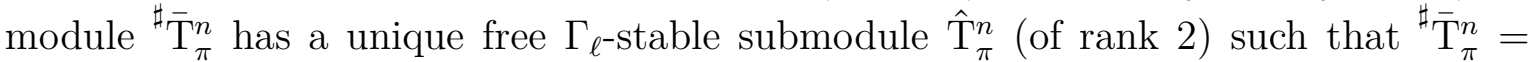
${ }^{\sharp} \overline{\mathrm{T}}_{\pi}^{n}\left[\ell^{2} \mid 1\right] \oplus \hat{\mathrm{T}}_{\pi}^{n}$. Moreover, since $\operatorname{tr}\left(\operatorname{Fr}_{\ell^{2}} ; \mathrm{V}_{\pi}\right) \bmod p \notin\left\{\ell^{2}+1,-\ell^{2}-1\right\}$, we have

$$
\left(\overline{\mathrm{T}}_{\sigma}^{n} \otimes_{\mathbb{Z} / p^{n}} \hat{\mathrm{T}}_{\pi}^{n}\right)\left[\ell^{2} \mid 1\right]=\operatorname{Hom}_{\mathbb{Z} / p^{n}}\left(\overline{\mathrm{T}}_{\sigma}^{n} \otimes_{\mathbb{Z} / p^{n}} \hat{\mathrm{T}}_{\pi}^{n}, \mathbb{Z} / p^{n}(1)\right)\left[\ell^{2} \mid 1\right]=0 .
$$

In particular, we have

$$
\mathrm{H}^{1}\left(\mathbb{Q}_{\ell}, \overline{\mathrm{T}}_{\sigma}^{n} \otimes_{\mathbb{Z} / p^{n}} \hat{\mathrm{T}}_{\pi}^{n}\right)=\mathrm{H}^{1}\left(\mathbb{Q}_{\ell^{2}}, \overline{\mathrm{T}}_{\sigma}^{n} \otimes_{\mathbb{Z} / p^{n}} \hat{\mathrm{T}}_{\pi}^{n}\right)=0 .
$$

Thus (1) follows. Note that the action of $\operatorname{Fr}_{\ell}$ on $\overline{\mathrm{T}}_{\sigma}^{n} \otimes_{\mathbb{Z} / p^{n}}{ }^{\sharp} \overline{\mathrm{T}}_{\pi}^{n}\left[\ell^{2} \mid 1\right]$ has eigenvalues $\{1,-1, \ell,-\ell\}$ which are distinct even modulo $p$. Therefore, $(2-5)$ follow by the same reason as Lemma 3.25. Since $p$ is odd, (6) is obvious.

Lemma 4.3. Let $v$ be a prime other than $p$.

(1) There is an integer $n_{v} \geq 0$ such that the image of the pairing (4.1) is annihilated by $p^{n_{v}}$ for every $n \geq 1$.

(2) The submodule $\mathrm{H}_{\mathrm{unr}}^{1}\left(\mathbb{Q}_{v}, \overline{\mathrm{T}}_{\sigma, \pi}^{n}\right)$ is its own annihilator under the pairing $\langle,\rangle_{v}$ (4.1). 
Proof. By Lemma 3.9, we have $\mathrm{H}^{1}\left(\mathbb{Q}_{v}, \mathrm{~V}_{\sigma, \pi}\right)=0$, which implies that $\mathrm{H}^{1}\left(\mathbb{Q}_{v}, \mathrm{~T}_{\sigma, \pi}\right)$ is torsion (and finitely generated). Since we have the short exact sequence

$$
\mathrm{H}^{1}\left(\mathbb{Q}_{v}, \mathrm{~T}_{\sigma, \pi}\right) \rightarrow \mathrm{H}^{1}\left(\mathbb{Q}_{v}, \overline{\mathrm{T}}_{\sigma, \pi}^{n}\right) \rightarrow \mathrm{H}^{2}\left(\mathbb{Q}_{v}, \mathrm{~T}_{\sigma, \pi}\right)_{\text {tor }}
$$

(1) follows. Part (2) is well-known.

Notation 4.4. Put $n_{\text {bad }}=\max \left\{n_{v} \mid v\right.$ is a prime dividing $\left.q N^{-} \mathcal{D}_{N^{+} M}\right\}$.

4.1.2. The case $v=p$. We start with some general discussion.

Definition 4.5 (see, for example, [BM02, §3.1]). Let $a \leq 0 \leq b$ be two integers such that $b-a \leq p-2$. Let $\mathrm{T}$ be a finite $\mathbb{Z}_{p}\left[\Gamma_{p}\right]$-module. We say that $\mathrm{T}$ is a torsion crystalline module with Hodge-Tate weights in $[a, b]$ if $\mathrm{T}=\mathrm{L} / \mathrm{L}^{\prime}$ where $\mathrm{L}^{\prime} \subset \mathrm{L}$ are stable lattices in a crystalline $p$-adic representation of $\Gamma_{p}$ with Hodge-Tate weights in $[a, b]$.

Let $\mathrm{T}$ be a finitely generated $\mathbb{Z}_{p}$-module with a continuous action of $\Gamma_{p}$. We say that $\mathrm{T}$ is a crystalline module with Hodge-Tate weights in $[a, b]$ if for all integers $n \geq 1$, the quotient $\mathrm{T} / p^{n} \mathrm{~T}$ is a torsion crystalline module with Hodge-Tate weights in $[a, b]$.

Definition 4.6 (see $[$ Niz93, $\S 4]$ ). Let $\mathrm{T}$ be a crystalline module of $\Gamma_{p}$ with Hodge-Tate weights in $[a, b]$. An element $s \in \mathrm{H}^{1}\left(\mathbb{Q}_{p}, \mathrm{~T}\right)$, represented by an extension

$$
0 \rightarrow \mathrm{T} \rightarrow \mathrm{T}_{s} \rightarrow \mathbb{Z}_{p} \rightarrow 0
$$

is finite if $\mathrm{T}_{s}$ is a crystalline module. Denote by $\mathrm{H}_{f}^{1}\left(\mathbb{Q}_{p}, \mathrm{~T}\right) \subset \mathrm{H}^{1}\left(\mathbb{Q}_{p}, \mathrm{~T}\right)$ the subset of finite elements which form a $\mathbb{Z}_{p}$-submodule. Moreover, the assignment $\mathrm{H}_{f}^{1}\left(\mathbb{Q}_{p},-\right)$ is functorial.

Remark 4.7. By the above definition, if $\mathrm{T}$ is a stable lattice in a crystalline $p$-adic representation $\mathrm{V}$ of $\Gamma_{p}$ with Hodge-Tate weights in $[a, b]$, then $\mathrm{H}_{f}^{1}\left(\mathbb{Q}_{p}, \mathrm{~T}\right)$ is the inverse image of $\mathrm{H}_{f}^{1}\left(\mathbb{Q}_{p}, \mathrm{~V}\right)$ under the natural map $\mathrm{H}^{1}\left(\mathbb{Q}_{p}, \mathrm{~T}\right) \rightarrow \mathrm{H}^{1}\left(\mathbb{Q}_{p}, \mathrm{~V}\right)$.

Lemma 4.8. Suppose that $p \geq 11$. Then $\mathrm{H}_{f}^{1}\left(\mathbb{Q}_{p}, \overline{\mathrm{T}}_{\sigma, \pi}^{n}\right)$ is its own annihilator under the local Tate pairing $\langle,\rangle_{p}(4.1)$.

Proof. We know that $\overline{\mathrm{T}}_{\sigma, \pi}^{n}$ is a torsion crystalline module of Hodge-Tate weights in $[-2,1]$. The lemma follows from [Niz93, Proposition 6.2] since $3 \leq(11-2) / 2$.

4.2. Integral Tate cycles. We study $\pi$-isotypic Tate cycles. Let $\ell$ be a strongly 1-admissible prime. Recall that we have a canonical isomorphism

$$
\mathrm{Z}^{0}\left(\mathcal{X}_{N^{+} M ; \mathbb{F}_{\ell^{2}}}^{\mathrm{ss}}\right) \simeq \mathbb{Z}\left[\mathcal{S}_{N^{+} M}^{\bullet} \sqcup \mathcal{S}_{N^{+} M}^{\circ}\right] \simeq \mathbb{Z}\left[\mathcal{S}_{N^{+} M}\right]^{\oplus 2}
$$

Put

$$
\mathrm{Z}_{\pi \mid N^{+} M}=\mathbb{Z}_{p}\left[\mathcal{S}_{N^{+} M}^{\bullet} \sqcup \mathcal{S}_{N^{+} M}^{\circ}\right] \cap\left(\mathbb{Q}_{p}\left[\mathcal{S}_{N^{+} M}\right]\left[\pi^{Q}\right]\right)^{\oplus 2},
$$

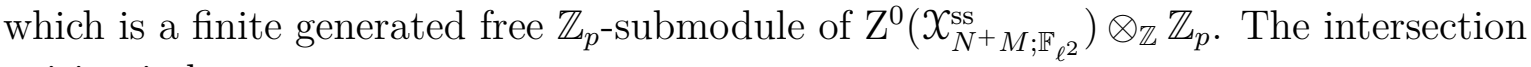
pairing induces a map

$$
\mathrm{H}_{\text {ét }}^{2}\left(X_{N^{+} M ; \mathbb{F}_{\ell}^{\text {ac }}}, \mathbb{Z}_{p}(1)\right)^{\mathbb{F}_{\ell^{2}}} \rightarrow \operatorname{Hom}\left(Z^{0}\left(X_{N^{+} M ; \mathbb{F}_{\ell^{2}}}^{\text {ss }}\right) \otimes_{\mathbb{Z}} \mathbb{Z}_{p}, \mathbb{Z}_{p}\right)
$$


Proposition 4.9. The restriction of (4.2) induces an isomorphism

$$
\mathrm{H}_{\text {ét }}^{2}\left(X_{N^{+} M ; \mathbb{F}_{\ell}^{\text {ac }}}, \mathbb{Z}_{p}(1)\right)^{\mathbb{F}_{\ell^{2}}} \cap \mathrm{H}_{\pi}\left(\mathcal{X}_{N^{+} M ; \mathbb{F}_{\ell}^{\mathrm{ac}}}\right) \stackrel{\sim}{\rightarrow} \operatorname{Hom}\left(\mathrm{Z}_{\pi \mid N^{+} M}, \mathbb{Z}_{p}\right),
$$

under which the action of $\mathrm{Fr}_{\ell}$ on the source coincides with the involution induced by switching two factors on the target.

Proof. This is a very special case of the main result of [TX14]. See [TX14, §1.1] for this particular case. In fact, the proposition is a direct consequence of Proposition 2.21 (4) together with some Hecke theoretical argument. Note that the reason we may replace the coefficient $\mathbb{Q}_{p}$ by $\mathbb{Z}_{p}$ is that $\operatorname{tr}\left(\mathrm{Fr}_{\ell^{2}} ; \mathrm{V}_{\pi}\right) \bmod p \notin\{2 \ell,-2 \ell\}$ in Definition $3.31(\mathrm{~S} 2)$.

In what follows, we will present an element of $Z_{\pi}$ as a pair of functions $f=\left(f^{\bullet}, f^{\circ}\right)$ on $\mathcal{S}_{N^{+} M}^{\bullet} \sqcup \mathcal{S}_{N^{+} M}^{\circ}$. In particular, we have the element

$$
f_{\nu^{\bullet}, \nu^{\circ}}:=\left(\nu^{\bullet} f_{\pi}^{(\mathfrak{d})}, \nu^{\circ} f_{\pi}^{(\mathfrak{d})}\right)=\left(\nu^{\bullet}\left(\gamma_{N^{+} M}^{\left(N^{+} M / \mathfrak{M}, \mathfrak{d}\right)}\right)^{*} f_{\pi}, \nu^{\circ}\left(\gamma_{N^{+} M}^{\left(N^{+} M / \mathfrak{M}, \mathfrak{d}\right)}\right)^{*} f_{\pi}\right)
$$

for $\nu^{\bullet}, \nu^{\circ} \in \mathbb{Z}_{p}$. We define

$$
\mathrm{Z}_{\pi}=\left\{f_{\nu^{\bullet}, \nu^{\circ}} \mid \nu^{\bullet}, \nu^{\circ} \in \mathbb{Z}_{p}\right\} \subset \mathrm{Z}_{\pi \mid N^{+} M}
$$

as a free submodule of rank 2 . Then the projection $\operatorname{Hom}\left(\mathrm{Z}_{\pi \mid N^{+} M}, \mathbb{Z}_{p}\right) \rightarrow \operatorname{Hom}\left(\mathrm{Z}_{\pi}, \mathbb{Z}_{p}\right)$ coincides with the map

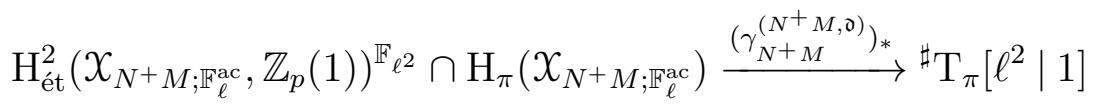

under (4.3). In particular, we have an isomorphism

$$
\mathrm{c}_{\ell}:{ }^{\sharp} \overline{\mathrm{T}}_{\pi}^{n}\left[\ell^{2} \mid 1\right] \stackrel{\sim}{\rightarrow} \operatorname{Hom}\left(\overline{\mathrm{Z}}_{\pi}^{n}, \mathbb{Z} / p^{n}\right)
$$

for $1 \leq n \leq \infty$.

4.3. Statement of congruence formulae. The following two theorems are the main technical results of the article. We recall the two maps $\mathrm{u}_{\ell}$ and $\mathbf{s}_{\ell}$ in (3.6).

Theorem 4.10. Suppose that Assumption $3.8(R 1-R 4)$ are satisfied and $\wp\left(N^{-}\right)$is even. Let $n \geq 1$ be an integer and $\ell_{1}, \ell_{2}$ be two distinct strongly $n$-admissible primes. Then

(1) $\operatorname{loc}_{\ell_{1}^{2}}\left(\bar{\Delta}_{\sigma, \pi}^{n}\right)$ belongs to $\mathrm{H}_{\mathrm{unr}}^{1}\left(\mathbb{Q}_{\ell_{1}^{2}}, \overline{\mathrm{T}}_{\sigma, \pi}^{n}\right) \simeq \mathrm{H}_{\mathrm{unr}}^{1}\left(\mathbb{Q}_{\ell_{1}^{2}}, \overline{\mathrm{T}}_{\sigma}^{n}\right) \otimes_{\mathbb{Z} / p^{n}}{ }^{\sharp} \overline{\mathrm{T}}_{\pi}^{n}\left[\ell_{1}^{2} \mid 1\right]$;

(2) as an element in $\operatorname{Hom}\left(\overline{\mathrm{Z}}_{\pi}^{n}, \mathbb{Z} / p^{n}\right)$, the map $\left(\mathrm{u}_{\ell_{1}} \otimes \mathrm{c}_{\ell_{1}}\right) \operatorname{loc}_{\ell_{1}^{2}}\left(\bar{\Delta}_{\sigma, \pi}^{n}\right)$ sends $f_{\nu^{\bullet}, \nu^{\circ}}$ to

$$
\left(\nu^{\bullet}+\epsilon_{\sigma}\left(\ell_{1}\right) \nu^{\circ}\right) \sum_{t \in \mathcal{T}_{N+M, N}-\ell_{1}}\left(\zeta_{N^{+} M, N^{-} \ell_{1}}^{*} f_{\pi}^{(\mathfrak{d})}\right)(t) \cdot\left(\left(\delta_{N^{+} M, N^{-} \ell_{1}}^{N^{+} M, d}\right)^{*} g_{\sigma \mid \ell_{1}}^{n}\right)(t)
$$

viewed as in $\mathbb{Z} / p^{n}$, where $\delta_{N^{+} M, N^{+} \ell_{1}}^{N^{+} M, d}$ is defined in Notation 2.2 (5); and $g_{\sigma \mid \ell_{1}}^{n}$ is defined in (3.10); 
(3) as an element in $\operatorname{Hom}\left(\bar{Z}_{\pi}^{n}, \mathbb{Z} / p^{n}\right)$, the map $\left(\mathbf{s}_{\ell_{2}} \otimes \mathrm{c}_{\ell_{2}}\right) \partial_{\ell_{2}^{2}} \operatorname{loc}_{\ell_{2}^{2}}\left(\bar{\Delta}_{\sigma, \pi \mid \ell_{1}, \ell_{2}}^{n}\right)$ sends $f_{\nu \bullet, \nu^{\circ}}$ to

$$
\begin{aligned}
& \left(\operatorname{tr}\left(\operatorname{Fr}_{\ell_{2}^{2}} ; \mathrm{V}_{\pi}\right)-2 \epsilon_{\sigma}\left(\ell_{2}\right) \ell_{2}\right)\left(\nu^{\circ}+\epsilon_{\sigma}\left(\ell_{2}\right) \nu^{\bullet}\right) \\
& \times \sum_{t \in \mathcal{T}_{N+M, N^{-} \ell_{1}}}\left(\zeta_{N^{+} M, N^{-} \ell_{1}}^{*} f_{\pi}^{(\mathfrak{d})}\right)(t) \cdot\left(\left(\delta_{N^{+} M, N^{-} \ell_{1}}^{N^{+} M, d}\right)^{*} g_{\sigma \mid \ell_{1}}^{n}\right)(t)
\end{aligned}
$$

viewed as in $\mathbb{Z} / p^{n}$.

Theorem 4.11. Suppose that Assumption $3.8\left(R 1-R_{4}\right)$ are satisfied and $\wp\left(N^{-}\right)$is odd. Let $n \geq 1$ be an integer and $\ell$ be a strongly $n$-admissible prime. Then as an element in $\operatorname{Hom}\left(\overline{\mathbf{Z}}_{\pi}^{n}, \mathbb{Z} / p^{n}\right)$, the map $\left(\mathbf{s}_{\ell} \otimes \mathbf{c}_{\ell}\right) \partial_{\ell^{2}} \operatorname{loc}_{\ell^{2}}\left(\bar{\Delta}_{\sigma, \pi \mid \ell}^{n}\right)$ sends $f_{\nu^{\bullet}, \nu^{\circ}}$ to

$$
\begin{aligned}
& \left(\operatorname{tr}\left(\operatorname{Fr}_{\ell^{2}} ; \mathrm{V}_{\pi}\right)-2 \epsilon_{\sigma}(\ell) \ell\right)\left(\nu^{\circ}+\epsilon_{\sigma}(\ell) \nu^{\bullet}\right) \\
& \times \sum_{t \in \mathcal{T}_{N^{+}} M, N^{-}}\left(\zeta_{N^{+} M, N^{-}}^{*} f_{\pi}^{(\mathfrak{d})}\right)(t) \cdot\left(\left(\delta_{N^{+} M, N^{-}}^{N^{+} M, d}\right)^{*} g_{\sigma}^{n}\right)(t)
\end{aligned}
$$

viewed as in $\mathbb{Z} / p^{n}$, where $g_{\sigma}^{n}$ is defined in (3.7).

Note that if $\ell$ is strongly 1-admissible, then $\operatorname{tr}\left(\mathrm{Fr}_{\ell^{2}} ; \mathrm{V}_{\pi}\right) \pm 2 \ell \in \mathbb{Z}_{p}^{\times}$by Definition 3.31 (S3). We will prove Theorem $4.10(1,2)$ at the end of $\$ 4.4$ and Theorem 4.10 (3), Theorem 4.11 at the end of $\$ 4.5$. In fact, among the three congruence formulae: Theorem 4.10 (2), (3), and Theorem 4.11, the latter two are proved by the same way. However, we would like to pack them according to the parity of $\wp\left(N^{-}\right)$as appeared above.

4.4. Computation of localization, I. Let $R$ be a discrete valuation ring in which $p$ is invertible. Let $k$ (resp. $\tilde{k}$ ) be its fraction (resp. residue) field. Let $X$ (resp. $Y$ ) be a smooth proper (resp. flat proper) scheme over $R$. Assume that the generic fiber $X_{k}$ (resp. $Y_{k}$ ) is smooth purely of dimension $2 d$ (resp. $2 d-1$ ) for some $d \geq 1$. Put $Z=Y \times_{\text {Spec } R} X$ with $\pi_{X}: Z \rightarrow X$ and $\pi_{Y}: Z \rightarrow Y$ the two projections.

For $1 \leq n \leq \infty$, denote by $\Theta^{n}$ the composition of the following maps:

$$
\begin{aligned}
\Theta^{n} & : \mathrm{H}^{1}\left(k, \mathrm{H}_{\text {ét }}^{4 d-1}\left(Z_{k^{\text {ac }}}, \mathbb{Z} / p^{n}(2 d)\right)\right) \\
& \rightarrow \mathrm{H}^{1}\left(k, \operatorname{Hom}\left(\mathrm{H}_{\text {ét }}^{2 d}\left(X_{k^{\mathrm{ac}}}, \mathbb{Z} / p^{n}(d)\right), \mathrm{H}_{\text {ét }}^{2 d-1}\left(Y_{k^{\mathrm{ac}}}, \mathbb{Z} / p^{n}(d)\right)\right)\right) \\
& \rightarrow \mathrm{H}^{1}\left(k, \operatorname{Hom}\left(\mathrm{H}_{\text {ét }}^{2 d}\left(X_{k^{\mathrm{ac}}}, \mathbb{Z} / p^{n}(d)\right)^{k}, \mathrm{H}_{\text {ét }}^{2 d-1}\left(Y_{k^{\mathrm{ac}}}, \mathbb{Z} / p^{n}(d)\right)\right)\right) \\
& \stackrel{\sim}{\rightarrow} \operatorname{Hom}\left(\mathrm{H}_{\text {ét }}^{2 d}\left(X_{k^{\mathrm{ac}}}, \mathbb{Z} / p^{n}(d)\right)^{k}, \mathrm{H}^{1}\left(k, \mathrm{H}_{\text {ét }}^{2 d-1}\left(Y_{k^{\mathrm{ac}}}, \mathbb{Z} / p^{n}(d)\right)\right)\right) \\
& \stackrel{\sim}{\rightarrow} \operatorname{Hom}\left(\mathrm{H}_{\text {ét }}^{2 d}\left(X_{\tilde{k}^{\text {ac }}}, \mathbb{Z} / p^{n}(d)\right)^{\tilde{k}}, \mathrm{H}^{1}\left(k, \mathrm{H}_{\text {ét }}^{2 d-1}\left(Y_{k^{\mathrm{ac}}}, \mathbb{Z} / p^{n}(d)\right)\right)\right) \\
& \rightarrow \operatorname{Hom}\left(\mathrm{CH}^{d}\left(X_{\tilde{k}}, \mathbb{Z} / p^{n}\right), \mathrm{H}^{1}\left(k, \mathrm{H}_{\text {ét }}^{2 d-1}\left(Y_{k^{\text {ac }}}, \mathbb{Z} / p^{n}(d)\right)\right)\right),
\end{aligned}
$$

where the first arrow is induced by Künneth decomposition and Poincaré duality; and the last arrow is induced by $\mathrm{cl}_{X_{\tilde{k}}}^{0}$. Here we recall the convention from $\S 1.6 .3$ that ${ }^{k}{ }^{k}$ is nothing but $-\Gamma_{k}$. 
4.4.1. Smooth reduction in general case. Now assume that $Y$ is also smooth over $R$, and hence $Z$ is smooth proper over $R$.

Lemma 4.12. Let $W \in \mathrm{Z}^{c}(Z)$ be a codimension c cycle on $Z$ such that its support $W^{\prime}$, equipped with the induced reduced structure, is normal and flat over $R$, and the class $\mathrm{cl}_{Z_{k}}\left(W_{k}\right)$ belongs to $\mathrm{H}_{\text {ét }}^{2 c}\left(Z_{k}, \mathbb{Z} / p^{n}(c)\right)^{0}$. Then

(1) the class $\mathrm{cl}_{Z_{\tilde{k}}}\left(W_{\tilde{k}}\right)$ belongs to $\mathrm{H}_{\text {ét }}^{2 c}\left(Z_{\tilde{k}}, \mathbb{Z} / p^{n}(c)\right)^{0}$;

(2) the class $\left[W_{k}\right]_{Z_{k}}^{n}$ belongs to $\mathrm{H}_{\mathrm{unr}}^{1}\left(k, \mathrm{H}_{\text {ét }}^{2 c-1}\left(Z_{k^{\text {ac }}}, \mathbb{Z} / p^{n}(c)\right)\right)$;

(3) $\left[W_{k}\right]_{Z_{k}}^{n}=\left[W_{\tilde{k}}\right]_{Z_{\tilde{k}}}^{n}$ under the canonical isomorphism

$$
\mathrm{H}_{\text {unr }}^{1}\left(k, \mathrm{H}_{\text {ét }}^{2 c-1}\left(Z_{k^{\text {ac }}}, \mathbb{Z} / p^{n}(c)\right)\right) \simeq \mathrm{H}^{1}\left(\tilde{k}, \mathrm{H}_{\text {ét }}^{2 c-1}\left(Z_{\tilde{k}^{\text {ac }}}, \mathbb{Z} / p^{n}(c)\right)\right) \text {. }
$$

This lemma holds for an arbitrary scheme $Z$ that is smooth proper over $R$, not necessarily of the form $X \times_{\operatorname{Spec} R} Y$.

Proof. For a noetherian scheme $S$, we denote by $S_{\mathrm{s}}$ the singular locus of $S_{\text {red }}$, which is a Zariski closed subset containing no generic point, equipped with the induced reduced structure.

We have $W_{\mathrm{s} ; k}^{\prime}=\left(W_{k}^{\prime}\right)_{\mathrm{s}}$. Define ${ }^{\mathrm{s}} W_{\tilde{k}}^{\prime}$ to be the union of $W_{\mathrm{s} ; \tilde{k}}^{\prime}$ and $\left(W_{\tilde{k}}^{\prime}\right)_{\mathrm{s}}$. Since $W^{\prime}$ is normal and flat proper over $R$, the subset ${ }^{\mathrm{s}} W_{\tilde{k}}^{\prime}$ is closed in $W_{\tilde{k}}^{\prime}$ of codimension everywhere at least 1 . Let $I$ (resp. $\tilde{I}$ ) be the (finite) set of irreducible components of $W^{\prime}\left(\right.$ resp. $\left.W_{\tilde{k}}^{\prime}\right)$. We have a natural specialization map sp: $\bigoplus_{I} \mathbb{Z} \rightarrow \bigoplus_{\tilde{I}} \mathbb{Z}$. If we regard the source (resp. target) as the subgroup of $\mathrm{Z}^{c}(Z)$ (resp. $\mathrm{Z}^{c}\left(Z_{\tilde{k}}\right)$ ) consisting of cycles supported on $W^{\prime}$ (resp. $\left.W_{\tilde{k}}^{\prime}\right)$, then sp sends $W$ to $W_{\tilde{k}}$. Thus, we have the following commutative diagram

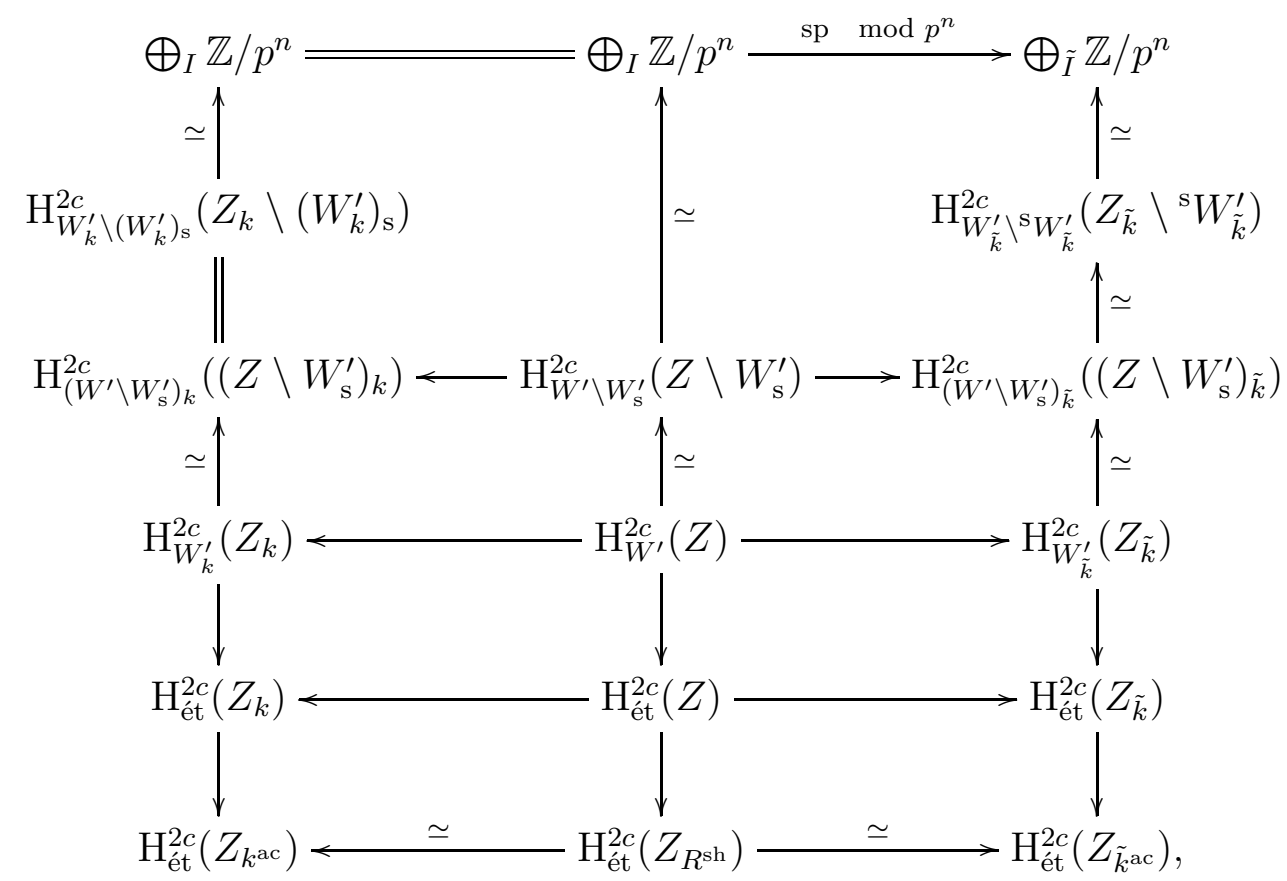

in which the coefficient sheaf for all étale cohomology groups is $\mathbb{Z} / p^{n}(c)$. We have that

- the last three vertical arrows are all restriction maps; 
- the three arrows toward the first row are all isomorphisms by purity;

- the remaining four upward arrows are all isomorphisms by semi-purity; and

- the two bottom arrows are isomorphisms since $Z$ is smooth proper over $R$, where $R^{\text {sh }}$ is the strict henselization of $R$.

Recall how we define the cycle class map. If we start from $W$, viewed as an element in the middle term $\bigoplus_{I} \mathbb{Z} / p^{n}$, and move along three routes (left-down, down, right-down) all the way down to the second last row, then what we obtain $\operatorname{are~} \mathrm{cl}_{Z_{k}}\left(W_{k}\right), \mathrm{cl}_{Z}(W)$ and $\mathrm{cl}_{Z_{\tilde{k}}}\left(W_{\tilde{k}}\right)$, respectively. All these classes map to 0 under the last vertical arrows which are restriction maps. The lemma then follows by the functoriality of Hochschild--Serre spectral sequences.

Proposition 4.13. Let $W \in \mathrm{Z}^{2 d}(Z)$ be a codimension $2 d$ cycle on $Z$ such that its support is normal and flat proper over $R$, and $\mathrm{cl}_{Z_{k}}\left(W_{k}\right) \in \mathrm{H}_{\text {ét }}^{4 d}\left(Z_{k}, \mathbb{Z} / p^{n}(2 d)\right)^{0}$. For $c \in \mathrm{CH}^{d}\left(X_{\tilde{k}}, \mathbb{Z} / p^{n}\right)$, the map $\Theta^{n}\left[W_{k}\right]_{Z_{k}}^{n}$ sends $c$ to

$$
\begin{aligned}
{\left[\left(\pi_{Y ; \tilde{k}}\right)_{*}\left(\pi_{X ; \tilde{k}}^{*} c . W_{\tilde{k}}\right)\right]_{Y_{\hat{k}}}^{n} } & \in \mathrm{H}^{1}\left(\tilde{k}, \mathrm{H}_{\text {ét }}^{2 d-1}\left(Y_{\tilde{k}^{\mathrm{ac}}}, \mathbb{Z} / p^{n}(d)\right)\right) \\
& \simeq \mathrm{H}_{\mathrm{unr}}^{1}\left(k, \mathrm{H}_{\text {ét }}^{2 d-1}\left(Y_{k^{\mathrm{ac}}}, \mathbb{Z} / p^{n}(d)\right)\right) \subset \mathrm{H}^{1}\left(k, \mathrm{H}_{\text {ét }}^{2 d-1}\left(Y_{k^{\mathrm{ac}}}, \mathbb{Z} / p^{n}(d)\right)\right) .
\end{aligned}
$$

Proof. By Lemma 4.12, we may replace $\Theta^{n}\left[W_{k}\right]_{Z_{k}}^{n}$ by $\Theta^{n}\left[W_{\tilde{k}}\right]_{Z_{\tilde{k}}}^{n}$. Moreover, it is clear that the Chow cycle $\pi_{Y *}\left(\pi_{X ; \tilde{k}}^{*} c . W_{\tilde{k}}\right)$ belongs to $\mathrm{CH}^{2 d}\left(Y_{\tilde{k}}, \mathbb{Z} / p^{n}\right)^{0}$. Therefore, the expression $\left[\left(\pi_{Y *}\left(\pi_{X ; \tilde{k}}^{*} c . W_{\tilde{k}}\right)\right]_{Y_{\tilde{k}}}^{n}\right.$ is well-defined.

By definition and Lemma 3.14, the composition of the following maps

$$
\begin{aligned}
& \mathrm{H}_{\text {ét }}^{4 d}\left(Z_{\tilde{k}}, \mathbb{Z} / p^{n}(2 d)\right)^{0} \otimes \mathrm{H}_{\text {ét }}^{2 d}\left(X_{\tilde{k}}, \mathbb{Z} / p^{n}(d)\right) \\
& \rightarrow \mathrm{H}^{1}\left(\tilde{k}, \mathrm{H}_{\text {ét }}^{4 d-1}\left(Z_{\tilde{k}^{\mathrm{ac}}}, \mathbb{Z} / p^{n}(2 d)\right)\right) \otimes \mathrm{H}_{\text {ét }}^{2 d}\left(X_{\tilde{k}^{\mathrm{ac}}}, \mathbb{Z} / p^{n}(d)\right)^{\tilde{k}} \\
& \rightarrow \mathrm{H}^{1}\left(\tilde{k}, \operatorname{Hom}\left(\mathrm{H}_{\text {ét }}^{2 d}\left(X_{\tilde{k}^{\mathrm{ac}}}, \mathbb{Z} / p^{n}(d)\right), \mathrm{H}_{\text {ét }}^{2 d-1}\left(Y_{\tilde{k}^{\mathrm{ac}}}, \mathbb{Z} / p^{n}(d)\right)\right) \otimes \mathrm{H}_{\text {ét }}^{2 d}\left(X_{\tilde{k}^{\mathrm{ac}}}, \mathbb{Z} / p^{n}(d)\right)^{\tilde{k}}\right. \\
& \rightarrow \operatorname{Hom}\left(\mathrm{H}_{\text {ét }}^{2 d}\left(X_{\tilde{k}^{\mathrm{ac}}}, \mathbb{Z} / p^{n}(d)\right)^{\tilde{k}}, \mathrm{H}^{1}\left(\tilde{k}, \mathrm{H}_{\text {ét }}^{2 d-1}\left(Y_{\tilde{k}^{\mathrm{ac}}}, \mathbb{Z} / p^{n}(d)\right)\right)\right) \otimes \mathrm{H}_{\text {ét }}^{2 d}\left(X_{\tilde{k}^{\mathrm{ac}}}, \mathbb{Z} / p^{n}(d)\right)^{\tilde{k}} \\
& \rightarrow \mathrm{H}^{1}\left(\tilde{k}, \mathrm{H}_{\text {ét }}^{2 d-1}\left(Y_{\tilde{k}^{\mathrm{ac}}}, \mathbb{Z} / p^{n}(d)\right)\right),
\end{aligned}
$$

in which the first arrow is $\xi_{Z_{\tilde{k}}}^{1} \otimes$ id and the last one is the evaluation map, coincides with the following composition of maps

$$
\begin{aligned}
& \mathrm{H}_{\text {ét }}^{4 d}\left(Z_{\tilde{k}}, \mathbb{Z} / p^{n}(2 d)\right)^{0} \otimes \mathrm{H}_{\text {ét }}^{2 d}\left(X_{\tilde{k}}, \mathbb{Z} / p^{n}(d)\right) \stackrel{\mathrm{id} \otimes \pi_{Z}^{*}}{\longrightarrow} \mathrm{H}_{\text {ét }}^{4 d}\left(Z_{\tilde{k}}, \mathbb{Z} / p^{n}(2 d)\right)^{0} \otimes \mathrm{H}_{\text {ét }}^{2 d}\left(Z_{\tilde{k}}, \mathbb{Z} / p^{n}(d)\right) \\
& \quad \stackrel{\cup}{\rightarrow} \mathrm{H}_{\text {ét }}^{6 d}\left(Z_{\tilde{k}}, \mathbb{Z} / p^{n}(3 d)\right)^{0} \stackrel{\pi_{Y *}}{\longrightarrow} \mathrm{H}_{\text {ét }}^{2 d}\left(Y_{\tilde{k}}, \mathbb{Z} / p^{n}(d)\right)^{0} \stackrel{\xi_{Y_{\tilde{k}}}^{1}}{\longrightarrow} \mathrm{H}^{1}\left(\tilde{k}, \mathrm{H}_{\text {ét }}^{2 d-1}\left(Y_{\tilde{k}^{\text {ac }}}, \mathbb{Z} / p^{n}(d)\right)\right) .
\end{aligned}
$$

The proposition follows since cycle class maps are multiplicative.

4.4.2. Proof of Theorem 4.10 (1, 2). Let $n \geq 1$ be an integer and $\ell=\ell_{1}$ be a strongly $n$-admissible prime. We apply the previous discussion to the case: $R=\mathbb{Z}_{\ell^{2}}$ (and hence $\left.k=\mathbb{Q}_{\ell^{2}}, \tilde{k}=\mathbb{F}_{\ell^{2}}\right), X=x_{N^{+} M ; \mathbb{Z}_{\ell^{2}}}, Y=y_{N^{+} M q, N^{-} ; \mathbb{Z}_{\ell^{2}}}$ (and hence $d=1$ ), and 
$W=\Delta_{N^{+} M, N^{-} ; \mathbb{Z}_{\ell^{2}}}^{\prime}$. Consider the following diagram

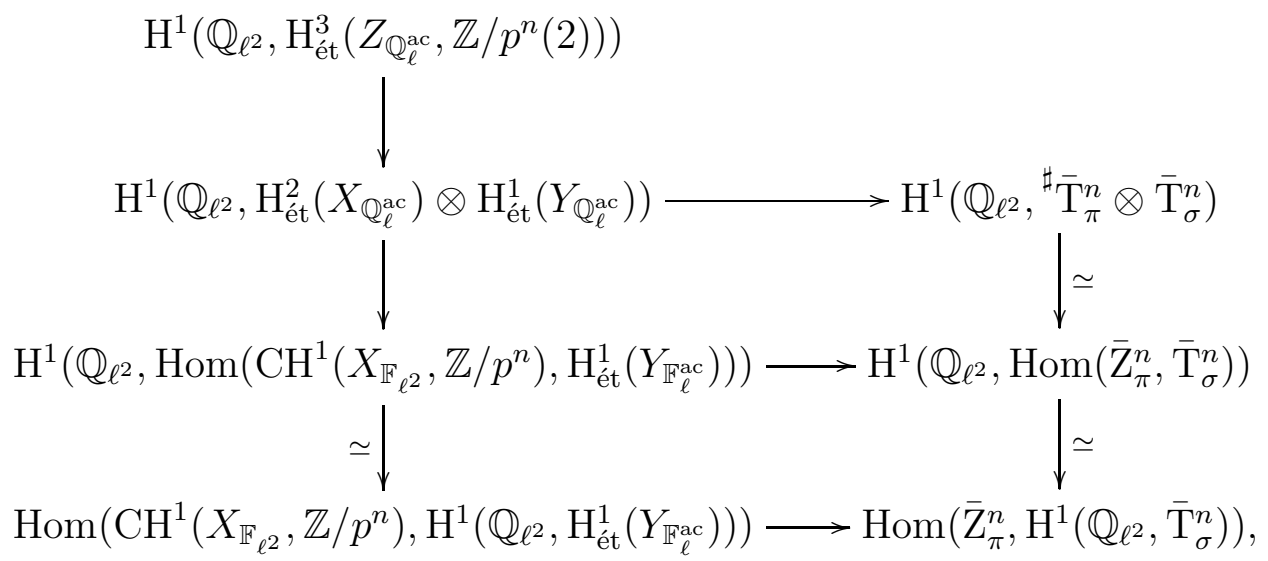

in which

- the coefficient sheaf of an étale cohomology group is $\mathbb{Z} / p^{n}(1)$ if not specified;

- the first horizontal arrow is induced by $\gamma_{N^{+} M}^{N^{+} M / \mathfrak{M}, \mathfrak{o}} \otimes \delta_{N^{+} M q, N^{-}}^{(M q, d)}$ and the projection to the $(\sigma, \pi)$-component;

- the first right vertical arrow is induced by (4.4), which is an isomorphism by Proposition 4.2 (1);

- the composition of all left vertical arrows is simply $\Theta^{n}(4.5)$.

Denote the composite map in the above diagram by

$$
\Theta_{\sigma, \pi}^{n}: \mathrm{H}^{1}\left(\mathbb{Q}_{\ell^{2}}, \mathrm{H}_{\text {ét }}^{3}\left(Z_{\mathbb{Q}_{\ell}^{\text {ac }}}, \mathbb{Z} / p^{n}(2)\right)\right) \rightarrow \operatorname{Hom}\left(\overline{\mathrm{Z}}_{\pi}^{n}, \mathrm{H}^{1}\left(\mathbb{Q}_{\ell^{2}}, \overline{\mathrm{T}}_{\sigma}^{n}\right)\right) .
$$

By construction, the element $\Theta_{\sigma, \pi}^{n}\left[W_{\mathbb{Q}_{\ell^{2}}}\right]_{Z_{\mathbb{Q}_{\ell} 2}}^{n}$ is nothing but $\mathrm{c}_{\ell} \operatorname{loc}_{\ell^{2}}\left(\bar{\Delta}_{\sigma, \pi}^{n}\right)$. The following lemma confirms Theorem 4.10 (1).

Lemma 4.14. The map $\Theta_{\sigma, \pi}^{n}\left[W_{\mathbb{Q}_{\ell^{2}}}\right]_{Z_{\mathbb{Q}^{2}}}^{n}$ factorizes through the natural map

$$
\mathbb{Z} / p^{n}\left[\mathcal{T}_{N^{+}, N^{-} \ell}\right]^{0} \rightarrow \mathrm{H}_{\mathrm{unr}}^{1}\left(\mathbb{Q}_{\ell^{2}}, \overline{\mathrm{T}}_{\sigma}^{n}\right) \rightarrow \mathrm{H}^{1}\left(\mathbb{Q}_{\ell^{2}}, \overline{\mathrm{T}}_{\sigma}^{n}\right)
$$

where the first arrow appears in (3.9).

Proof. This is a corollary of Proposition 4.13. Indeed, combining with Lemma 2.23, Proposition 2.24 and the definition of $\boldsymbol{\Delta}_{N^{+} M, N^{-}}^{\prime}$, we have for $f_{\nu^{\bullet}, \nu^{\circ}} \in \bar{Z}_{\pi}^{n}$,

$$
\begin{aligned}
& \pi_{Y *}\left(\pi_{X ; \tilde{k}}^{*} f_{\nu^{\bullet}, \nu^{\circ}} \cdot W_{\tilde{k}}\right) \\
& \quad=\frac{1}{q+1-\operatorname{tr}\left(\mathrm{Fr}_{q} ; \mathrm{V}_{\sigma}\right)}\left(\zeta_{N^{+} M, N^{-} \ell} \circ\left(\nu^{\bullet} \mathrm{id}+\nu^{\circ} \mathrm{op}_{\ell}\right) \circ\left(\delta_{N^{+} M q, N^{-} \ell}^{(q, 1)}-\delta_{N^{+} M q, N^{-} \ell}^{(q, q)}\right)\right)^{*} f_{\pi}^{(\mathfrak{d})}
\end{aligned}
$$

viewed as an element in $\mathbb{Z} / p^{n}\left[\mathcal{T}_{N^{+} M q, N^{-} \ell}\right]^{0} \subset \mathrm{CH}^{1}\left(Y_{\mathbb{F}_{\ell^{2}}}, \mathbb{Z} / p^{n}\right)^{0}$. The lemma follows by taking the projection $\delta_{N^{+} M q, N^{-}}^{(M, d)}$. 
From the above proof, we have

$$
\begin{aligned}
& \mathrm{u}_{\ell} \Theta_{\sigma, \pi}^{n}\left[W_{\mathbb{Q}_{\ell^{2}}}\right]_{Z_{\mathbb{Q}_{\ell^{2}}}^{n}}^{n}\left(f_{\nu^{\bullet}, \nu^{\circ}}\right) \\
& =\frac{1}{q+1-\operatorname{tr}\left(\operatorname{Fr}_{q} ; \mathrm{V}_{\sigma}\right)} \sum_{t \in \mathcal{T}_{N^{+} M q, N^{-} \ell}}\left(\left(\delta_{N^{+} M q, N^{-\ell}}^{(M q, d)}\right)^{*} g_{\sigma \mid \ell}^{n}\right)(t) \\
& \times\left(\left(\zeta_{N^{+} M, N^{-} \ell} \circ\left(\nu^{\bullet} \mathrm{id}+\nu^{\circ} \mathrm{op}_{\ell}\right) \circ\left(\delta_{N^{+} M q, N^{-} \ell}^{(q, 1)}-\delta_{N^{+} M q, N^{-} \ell}^{(q, q)}\right)\right)^{*} f_{\pi}^{(\mathfrak{d})}\right)(t) \\
& =\sum_{t \in \mathcal{T}_{N^{+} M, N^{-} \ell}}\left(\left(\delta_{N^{+} M, N^{-} \ell}^{(M, d)}\right)^{*} g_{\sigma \mid \ell}^{n}\right)(t) \cdot\left(\left(\zeta_{N^{+} M, N^{-} \ell} \circ\left(\nu^{\bullet} \mathrm{id}+\nu^{\circ} \mathrm{op}_{\ell}\right)\right)^{*} f_{\pi}^{(\mathfrak{d})}\right)(t) .
\end{aligned}
$$

Theorem 4.10 (2) follows since $g_{\sigma \mid \ell}^{n} \circ \mathrm{op}_{\ell}=\epsilon_{\sigma}(\ell) g_{\sigma \mid \ell}^{n}$.

4.5. Computation of localization, II. We keep the notation from the previous section. In addition, we assume that $R$ is a strictly henselian discrete valuation ring, and $d=1$. In particular, the morphism $f: Y \rightarrow \operatorname{Spec} R$ is a flat proper curve with smooth generic fiber. Let $n \geq 1$ be an integer.

\subsubsection{SNC reduction in general case.}

Definition 4.15. We say that a flat proper curve $f: Y \rightarrow \operatorname{Spec} R$ is an $S N C$ curve if

(1) $f_{*} \mathscr{O}_{Y}=\mathscr{O}_{\mathrm{Spec} R}$

(2) $Y$ is regular and $Y_{k}$ is smooth;

(3) all irreducible components of $Y_{\tilde{k}}$ are smooth and their intersection points are nodal.

Let $Y / R$ be an SNC curve. In this paragraph, we recall some facts from [BLR90, $\$ 9.5$ \& 9.6]. Denote by $\mathrm{Pic}_{Y / R}$ the relative Picard functor of $Y / R, \mathrm{P}_{Y / R}$ the open subfunctor of $\mathrm{Pic}_{Y / R}$ defined by line bundles of total degree 0. By [BLR90, §9.5, Theorem 4], the largest separated quotient $\mathrm{J}_{Y / R}$ of $\mathrm{P}_{Y / R}$ is representable, which is in fact a Néron model of the Jacobian of $Y_{k}$ over Spec $R$. Let $\Phi_{Y / R}$ be the group of connected components of $\mathrm{J}_{Y / R ; \tilde{k}}$. Denote by $\left\{Y_{i}\right\}_{i \in I}$ the set of irreducible components of $Y_{\tilde{k}}$. Consider maps

$$
\mathbb{Z}[I] \stackrel{\alpha}{\rightarrow} \mathbb{Z}[I] \stackrel{\operatorname{deg}}{\rightarrow} \mathbb{Z}
$$

where $\alpha$ is induced by the intersection matrix $\left(Y_{i} . Y_{j}\right)_{i, j \in I}$ and deg is the degree map. Then $\Phi_{Y / R}$ is canonically isomorphic to Ker deg/Im $\alpha$. The projection map $\phi: \mathrm{P}_{Y / R}(\tilde{k}) \rightarrow \Phi_{Y / R}$ is computed by the formula $\phi(L)=\left(L . Y_{i}\right)_{i \in I}$ for every line bundle $L$ on $Y_{\tilde{k}}$ of total degree 0 . We also have a reduction map $\mathrm{J}_{Y / R}(k) \rightarrow \Phi_{Y / R}$. Denote by $\operatorname{Pic}_{Y / R}(\tilde{k})_{n}$ the subset of $L \in \operatorname{Pic}_{Y / R}(\tilde{k})$ such that $p^{n} \mid \sum_{i \in I} L . Y_{i}$. Then the natural map

$$
\mathrm{P}_{Y / R}(\tilde{k}) / p^{n} \mathrm{P}_{Y / R}(\tilde{k}) \rightarrow \operatorname{Pic}_{Y / R}(\tilde{k})_{n} / p^{n} \operatorname{Pic}_{Y / R}(\tilde{k})
$$

is a bijection. Therefore, the map $\phi$ induces the following map

$$
\phi_{n}: \operatorname{Pic}_{Y / R}(\tilde{k})_{n} / p^{n} \operatorname{Pic}_{Y / R}(\tilde{k}) \rightarrow \Phi_{Y / R} / p^{n} \Phi_{Y / R} .
$$

On the other hand, we have the Kummer map

$$
\mathrm{P}_{Y / R}(k) / p^{n} \mathrm{P}_{Y / R}(k)=\mathrm{J}_{Y / R}(k) / p^{n} \mathrm{~J}_{Y / R}(k) \rightarrow \mathrm{H}^{1}\left(k, \mathrm{~J}_{Y / R}\left[p^{n}\right]\left(k^{\mathrm{ac}}\right)\right) .
$$


If we denote by $\operatorname{Pic}_{Y / R}(k)_{n}$ the subset of $L \in \operatorname{Pic}_{Y / R}(k)$ with degree divisible by $p^{n}$, then the natural map

$$
\mathrm{P}_{Y / R}(k) / p^{n} \mathrm{P}_{Y / R}(k) \rightarrow \operatorname{Pic}_{Y / R}(k)_{n} / p^{n} \operatorname{Pic}_{Y / R}(k)
$$

is a bijection. Therefore, the above Kummer map induces the following map

$$
\operatorname{Pic}_{Y / R}(k)_{n} / p^{n} \operatorname{Pic}_{Y / R}(k) \rightarrow \mathrm{H}^{1}\left(k, \mathrm{~J}_{Y / R}\left[p^{n}\right]\left(k^{\mathrm{ac}}\right)\right)
$$

Lemma 4.16. The map (4.7) factorizes through the (surjective) reduction map

$$
\operatorname{Pic}_{Y / R}(k)_{n} / p^{n} \operatorname{Pic}_{Y / R}(k) \simeq \mathrm{J}_{Y / R}(k) / p^{n} \mathrm{~J}_{Y / R}(k) \rightarrow \Phi_{Y / R} / p^{n} \Phi_{Y / R} .
$$

In particular, we have a natural map $\kappa_{n}: \Phi_{Y / R} / p^{n} \Phi_{Y / R} \rightarrow \mathrm{H}^{1}\left(k, \mathrm{~J}_{Y / R}\left[p^{n}\right]\left(k^{\mathrm{ac}}\right)\right)$.

Proof. Denote by $\mathrm{J}_{Y / R}^{0}$ the identity component of $\mathrm{J}_{Y / R}$. Then we have an exact sequence

$$
0 \rightarrow \mathrm{J}_{Y / R}^{0}(R) \rightarrow \mathrm{J}_{Y / R}(k) \rightarrow \Phi_{Y / R} \rightarrow 0 .
$$

Since $p$ is invertible on $R$, the multiplication morphism $p^{n}: \mathrm{J}_{Y / R}^{0} \rightarrow \mathrm{J}_{Y / R}^{0}$ is étale and surjective. Thus one has the Kummer map for $\mathrm{J}_{Y / R}^{0}$ as well, which yields the following commutative diagram

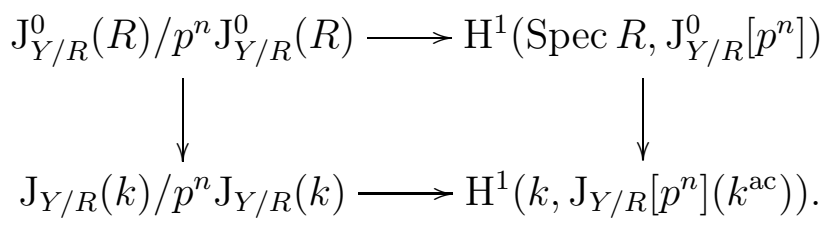

Since $R$ is strictly henselian, the cohomology $\mathrm{H}^{1}\left(\operatorname{Spec} R, \mathrm{~J}_{Y / R}^{0}\left[p^{n}\right]\right)$ vanishes. The lemma follows since the image of the map $\mathrm{J}_{Y / R}^{0}(R) / p^{n} \mathrm{~J}_{Y / R}^{0}(R) \rightarrow \mathrm{J}_{Y / R}(k) / p^{n} \mathrm{~J}_{Y / R}(k)$ coincides with the kernel of $\mathrm{J}_{Y / R}(k) / p^{n} \mathrm{~J}_{Y / R}(k) \rightarrow \Phi_{Y / R} / p^{n} \Phi_{Y / R}$.

Denote by $\widehat{\Theta}^{n}$ the composition of the following maps:

$$
\begin{aligned}
& \widehat{\Theta}^{n}: \mathrm{H}^{1}\left(k, \mathrm{H}_{\text {ét }}^{3}\left(Z_{k^{\mathrm{ac}}}, \mathbb{Z} / p^{n}(2)\right)\right) \\
& \rightarrow \mathrm{H}^{1}\left(k, \operatorname{Hom}\left(\mathrm{H}_{\text {ét }}^{2}\left(X_{k^{\mathrm{ac}}}, \mathbb{Z} / p^{n}(1)\right), \mathrm{H}_{\text {ét }}^{1}\left(Y_{k^{\mathrm{ac}}}, \mathbb{Z} / p^{n}(1)\right)\right)\right) \\
& \stackrel{\sim}{\rightarrow} \mathrm{H}^{1}\left(k, \operatorname{Hom}\left(\mathrm{H}_{\text {ét }}^{2}\left(X_{k^{\mathrm{ac}}}, \mathbb{Z} / p^{n}(1)\right), \mathrm{J}_{Y, R}\left[p^{n}\right]\left(k^{\mathrm{ac}}\right)\right)\right) \\
& \stackrel{\sim}{\rightarrow} \operatorname{Hom}\left(\mathrm{H}_{\text {ét }}^{2}\left(X_{\tilde{k}}, \mathbb{Z} / p^{n}(1)\right), \mathrm{H}^{1}\left(k, \mathrm{~J}_{Y, R}\left[p^{n}\right]\left(k^{\mathrm{ac}}\right)\right)\right) \\
& \rightarrow \operatorname{Hom}\left(\mathrm{CH}^{1}\left(X_{\tilde{k}}, \mathbb{Z} / p^{n}\right), \mathrm{H}^{1}\left(k, \mathrm{~J}_{Y, R}\left[p^{n}\right]\left(k^{\mathrm{ac}}\right)\right)\right),
\end{aligned}
$$

where we recall that $X / R$ is smooth proper.

Let $W=\sum a_{i} W_{i}$ be a cycle of $Z$ such that each $W_{i}$ is the graph of a finite flat morphism $w_{i}: Y \rightarrow X$, such that $W_{k} \in \mathrm{CH}^{2}\left(Z_{k}, \mathbb{Z} / p^{n}\right)^{0}$. In particular, we have the element $\left[W_{k}\right]_{Z_{k}}^{n} \in \mathrm{H}^{1}\left(k, \mathrm{H}_{\text {ét }}^{3}\left(Z_{k^{\text {ac }}}, \mathbb{Z} / p^{n}(2)\right)\right)$.

Proposition 4.17. For $L \in \mathrm{CH}^{1}\left(X_{\tilde{k}}, \mathbb{Z} / p^{n}\right)$, the element $\sum a_{i} \cdot w_{i}^{*} L$ is naturally in the quotient group $\operatorname{Pic}_{Y / R}(\tilde{k})_{n} / p^{n} \operatorname{Pic}_{Y / R}(\tilde{k})$, and moreover

$$
\widehat{\Theta}^{n}\left[W_{k}\right]_{Z_{k}}^{n}(L)=\kappa_{n} \phi_{n}\left(\sum a_{i} \cdot w_{i}^{*} L\right) .
$$


Proof. The first statement is immediate since $W_{k} \in \mathrm{CH}^{2}\left(Z_{k}, \mathbb{Z} / p^{n}\right)^{0}$ and by the compatibility of cycle class maps under specialization.

Since $\mathrm{H}_{\text {ét }}^{2}\left(X_{\tilde{k}}, \mathbb{Z} / p^{n}(1)\right) \simeq \mathrm{H}_{\text {ét }}^{2}\left(X, \mathbb{Z} / p^{n}(1)\right)$, we may regard $\operatorname{cl}_{X_{\tilde{k}}}(L)$ as an element in $\mathrm{H}_{\text {ét }}^{2}\left(X, \mathbb{Z} / p^{n}(1)\right)$, denoted by a new notation $[L]$ to distinguish. Denote by $[L]_{k}$ the restriction of $[L]$ in $\mathrm{H}_{\text {ét }}^{2}\left(X_{k}, \mathbb{Z} / p^{n}(1)\right)$. Then $\sum a_{i} \cdot w_{i ; k}^{*}[L]_{k}$ belongs to $\mathrm{H}_{\text {ét }}^{2}\left(Y_{k}, \mathbb{Z} / p^{n}(1)\right)^{0}$.

By a similar argument in the proof of Proposition 4.13, we find that

$$
\widehat{\Theta}^{n}\left[W_{k}\right]_{Z_{k}}^{n}(L)=\xi_{Z_{k}}^{1}\left(\sum a_{i} \cdot w_{i ; k}^{*}[L]_{k}\right) .
$$

Note that the following diagram

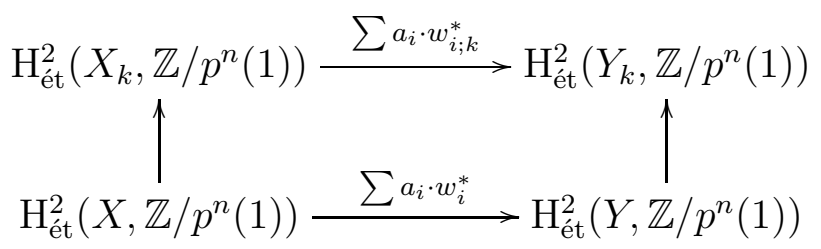

is commutative. Therefore, (4.9) is the restriction of $\sum a_{i} \cdot w_{i}^{*}[L] \in \mathrm{H}_{\text {êt }}^{2}\left(Y, \mathbb{Z} / p^{n}(1)\right)$ to $Y_{k}$, which belongs to $\mathrm{H}_{\text {ét }}^{2}\left(Y_{k}, \mathbb{Z} / p^{n}(1)\right)^{0}$, followed by $\xi_{Y_{k}}^{1}$. The proposition follows from the lemma below.

Lemma 4.18. Let $Y$ be an $S N C$ curve over $R$. Denote by $\mathrm{H}_{\text {ét }}^{2}\left(Y, \mathbb{Z} / p^{n}(1)\right)^{0}$ the inverse image of $\mathrm{H}_{\text {ét }}^{2}\left(Y_{k}, \mathbb{Z} / p^{n}(1)\right)^{0}$ under the restriction map. We have the following commutative diagram

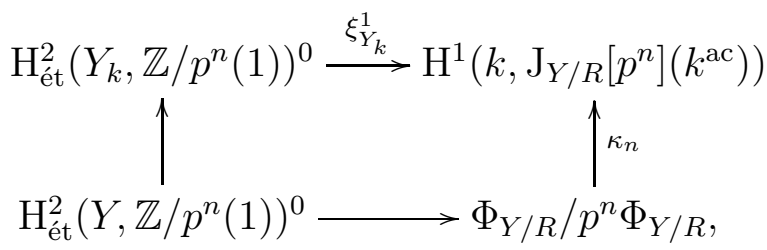

in which the bottom arrow sends $c \in \mathrm{H}_{\text {ét }}^{2}\left(Y, \mathbb{Z} / p^{n}(1)\right)^{0}$ to $\left(\left.\operatorname{deg} c\right|_{Y_{i}} \bmod p^{n}\right)_{i \in I}$ which is naturally an element in $\Phi_{Y / R} / p^{n} \Phi_{Y / R}$.

Proof. By [SS10, Theorem 5.1], the cycle class map

$$
\mathrm{cl}_{Y}: \mathrm{Z}^{1}(Y) \otimes_{\mathbb{Z}} \mathbb{Z} / p^{n} \rightarrow \mathrm{H}_{\text {ét }}^{2}\left(Y, \mathbb{Z} / p^{n}(1)\right)
$$

is surjective. Denote by $\mathrm{Z}^{1}\left(Y, \mathbb{Z} / p^{n}\right)^{0}$ the inverse image of $\mathrm{H}_{\text {ét }}^{2}\left(Y, \mathbb{Z} / p^{n}(1)\right)^{0}$ and $j: Y_{k} \rightarrow$ $Y$ the open immersion. For every $C \in \mathrm{Z}^{1}\left(Y, \mathbb{Z} / p^{n}\right)^{0}$, we may write $C=C_{h}+C_{v}$ where $C_{v}$ is vertical and $C_{h}$ is horizontal. We have that $j^{*} \operatorname{cl}_{Y}\left(C_{v}\right)=0$ and $\operatorname{cl}_{Y}\left(C_{v}\right)$ is mapped to 0 (even) in $\Phi_{Y / R}$. For $C_{h}$, the restriction $j^{*} C_{h}$ is an element in $\operatorname{Pic}_{Y / R}(k)$. Similarly to the proof of Lemma 4.12, we have $\operatorname{cl}_{Y_{k}}\left(j^{*} C_{h}\right)=j^{*} \mathrm{cl}_{Y}\left(C_{h}\right)$. In particular, the element $j^{*} C_{h}$ has degree divisible by $p^{n}$. The rest follows from Lemma 4.16.

4.5.2. Proof of Theorem 4.10 (3). Let $n \geq 1$ be an integer. We consider first the case where $\wp\left(N^{-}\right)$is even. Let $\ell_{1}, \ell_{2}$ be two distinct strongly $n$-admissible primes. We apply the previous discussion to the case: $R=\mathrm{W}\left(\mathbb{F}_{\ell_{2}}^{\mathrm{ac}}\right)\left(k=R \otimes_{\mathbb{Z}} \mathbb{Q}\right.$ and $\left.\tilde{k}=\mathbb{F}_{\ell_{2}}^{\mathrm{ac}}\right)$, 
$X=X_{N^{+} M ; R}, Y=y_{N^{+} M q, N^{-} \ell_{1} \ell_{2} ; R}$, and $W=\Delta_{N^{+} M, N^{-} \ell_{1} \ell_{2} ; R}^{\prime}$. Note that from (3.12), we have

$$
W=\frac{1}{q+1-\operatorname{tr}\left(\operatorname{Fr}_{q} ; \mathrm{V}_{\sigma}\right)} W^{\dagger}-\frac{1}{q+1-\operatorname{tr}\left(\mathrm{Fr}_{q} ; \mathrm{V}_{\sigma}\right)} W^{\ddagger},
$$

where $W^{\dagger}$ (resp. $\left.W^{\ddagger}\right)$ is the graph of $\zeta_{N^{+} M q, N^{-} \ell_{1} \ell_{2} ; R}^{\dagger}\left(\right.$ resp. $\left.\zeta_{N^{+} M q, N^{-} \ell_{1} \ell_{2} ; R}^{\ddagger}\right)$.

Put $Y_{1}=y_{N^{+}, N^{-} \ell_{1} \ell_{2} ; k}$ for short, and $\mathrm{J}_{1}$ the Jacobian of $Y_{1}$. Similarly to (4.6), we have the following commutative diagram.

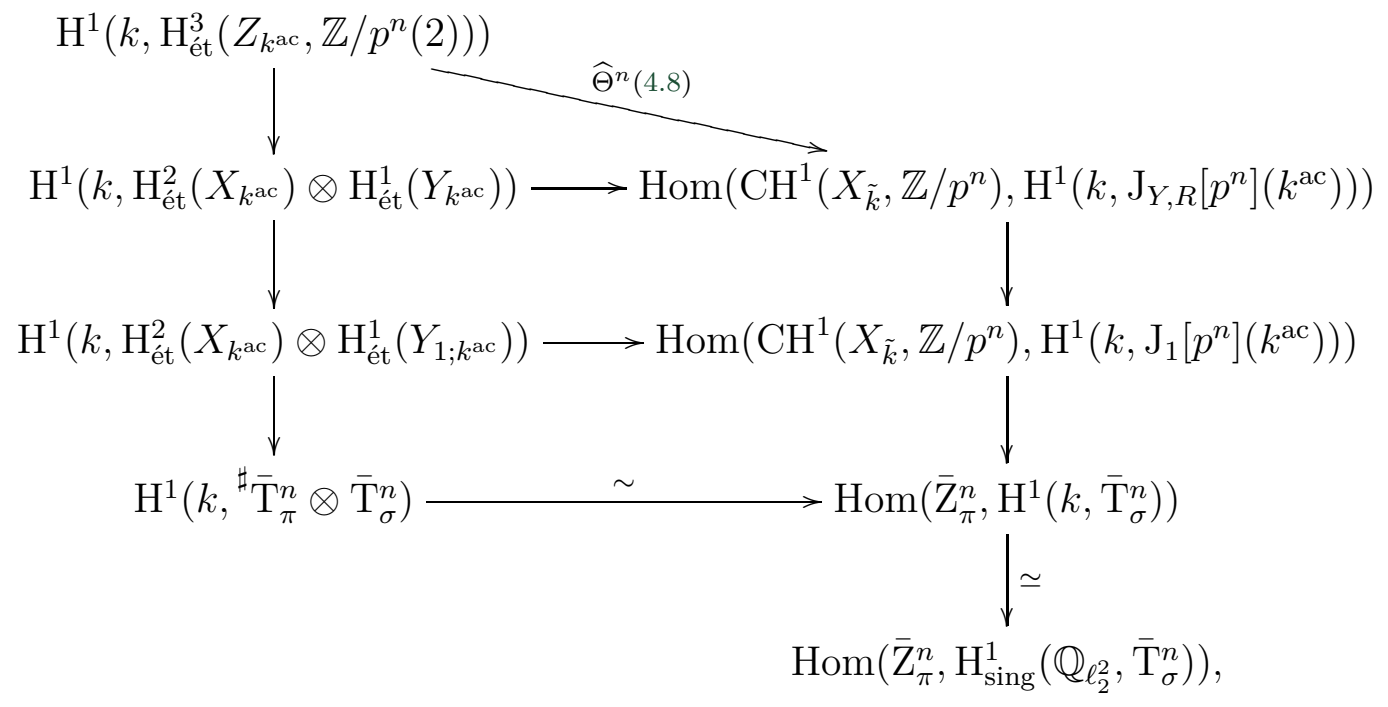

in which

- the coefficient sheaf of an étale cohomology group is $\mathbb{Z} / p^{n}(1)$ if not specified;

- the two vertical arrows from the second row are both induced by $\delta_{N^{+} M q, N^{-} \ell_{1} \ell_{2}}^{(M q}$;

- the two vertical arrows from the third row are both induced by $\gamma_{N^{+} M}^{\left(N^{+} M / \mathfrak{M}, \mathfrak{d}\right)}$, the projection to the $\pi$-component, and $\psi_{\ell_{1}, \ell_{2}}^{n}(3.11)$.

Denote the composite map in the above diagram by

$$
\widehat{\Theta}_{\sigma, \pi}^{n}: \mathrm{H}^{1}\left(k, \mathrm{H}_{\text {ét }}^{3}\left(Z_{k^{\mathrm{ac}}}, \mathbb{Z} / p^{n}(2)\right)\right) \rightarrow \operatorname{Hom}\left(\overline{\mathrm{Z}}_{\pi}^{n}, \mathrm{H}_{\text {sing }}^{1}\left(\mathbb{Q}_{\ell_{2}^{2}}, \overline{\mathrm{T}}_{\sigma}^{n}\right)\right) .
$$

By Proposition 4.17, for $f \in \mathbb{Z} / p^{n}\left[\mathcal{S}_{N^{+} M} \sqcup \mathcal{S}_{N^{+} M}^{\circ}\right]$, we have

$$
\widehat{\Theta}^{n}\left[W_{k}\right]_{Z_{k}}^{n}(f)=\frac{1}{q+1-\operatorname{tr}\left(\mathrm{Fr}_{q} ; \mathrm{V}_{\sigma}\right)} \kappa_{n} \phi_{n}\left(\zeta_{N^{+} M q, N^{-} \ell_{1} \ell_{2} ; \tilde{k}}^{\dagger *} f-\zeta_{N^{+} M q, N^{-} \ell_{1} \ell_{2} ; \tilde{k}}^{\dagger *} f\right) .
$$

Note that we have a natural multi-degree map

$$
\operatorname{Pic}_{Y / R}(\tilde{k}) \rightarrow \mathbb{Z}\left[\mathcal{T}_{N^{+} M q, N^{-} \ell_{1}}^{\bullet}\right] \oplus \mathbb{Z}\left[\mathcal{T}_{N^{+} M q, N^{-} \ell_{1}}^{\circ}\right]
$$

which induces a map

$$
\hat{\phi}_{n}: \operatorname{Pic}_{Y / R}(\tilde{k}) / p^{n} \operatorname{Pic}_{Y / R}(\tilde{k}) \rightarrow \mathbb{Z} / p^{n}\left[\mathcal{T}_{N^{+} M q, N^{-} \ell_{1}}^{\bullet}\right] \oplus \mathbb{Z} / p^{n}\left[\mathcal{T}_{N^{+} M q, N^{-} \ell_{1}}^{\circ}\right]
$$


rendering the following diagram

$$
\begin{aligned}
& \operatorname{Pic}_{Y / R}(\tilde{k})_{n} / p^{n} \operatorname{Pic}_{Y / R}(\tilde{k}) \stackrel{\hat{\phi}_{n}}{\longrightarrow} \mathbb{Z} / p^{n}\left[\mathcal{T}_{N^{+} M q, N^{-} \ell_{1}} \sqcup \mathcal{T}_{N^{+} M q, N^{-} \ell_{1}}^{\circ}\right]^{0} \\
& \phi_{n} \downarrow \quad \mid \phi_{N^{+}, N^{-} \ell_{1} \ell_{2}}^{\left(\ell_{2}\right)} \bmod p^{n} \\
& \Phi_{Y / R} / p^{n} \Phi_{Y / R}=\Phi_{N^{+} M q, N^{-} \ell_{1} \ell_{2}}^{\left(\ell_{2}\right.} / p^{n} \Phi_{N^{+} M q, N^{-} \ell_{1} \ell_{2}}^{\left(\ell_{2}\right)}
\end{aligned}
$$

commute, where $\phi_{N^{+}, N^{-} \ell_{1} \ell_{2}}^{\left(\ell_{2}\right)}$ is the map in Proposition 3.28.

By the definition of $\phi_{n}$, Remark 2.22 (2), Proposition 2.21 (4), and Proposition 2.25, we have

$$
\begin{array}{r}
\hat{\phi}_{n}\left(\zeta_{N^{+} M q, N^{-} \ell_{1} \ell_{2} ; \hat{k}}^{\dagger *} f\right)=\left(\delta_{N^{+} M q, N^{-} \ell_{1}}^{(q, 1)}\right)^{*} \zeta_{N^{+} M, N^{-} \ell_{1}}^{\left(\ell_{2}\right.} \\
\left(\left(\gamma_{N^{+}, M \ell_{2}}^{\left(\ell_{2}\right)}\right)_{*}\left(\gamma_{N^{+} M \ell_{2}}^{\left(\ell_{2}, \ell_{2}\right)}\right)^{\circ}-2 \ell_{2} f^{\bullet},\left(\gamma_{N^{+} M q \ell_{2}}^{\left(\ell_{2}, \ell_{2}\right)} *\left(\gamma_{N^{+} M q \ell_{2}}^{\left(\ell_{2}, 1\right)}\right)^{*} f^{\bullet}-2 \ell_{2} f^{\circ}\right) ;\right.
\end{array}
$$

and

$$
\begin{array}{r}
\hat{\phi}_{n}\left(\zeta_{N^{+} M q, N^{-} \ell_{1} \ell_{2} ; k^{*}}^{\ddagger *}\right)=\left(\delta_{N^{+} M q, N^{-} \ell_{1}}^{(q, q)}\right)^{*} \zeta_{N^{+} M, N^{-} \ell_{1}}^{*} \\
\left(\left(\gamma_{N^{+} M \ell_{2}}^{\left(\ell_{2}, 1\right)}\right)_{*}\left(\gamma_{N^{+} M \ell_{2}}^{\left(\ell_{2}, \ell_{2}\right)}\right)^{*} f^{\circ}-2 \ell_{2} f^{\bullet},\left(\gamma_{N^{+} M q \ell_{2}}^{\left(\ell_{2}, \ell_{2}\right)}\right)_{*}\left(\gamma_{N^{+} M q \ell_{2}}^{\left(\ell_{2}, 1\right)}\right)^{*} f^{\bullet}-2 \ell_{2} f^{\circ}\right) .
\end{array}
$$

Now suppose that $f=f_{\nu^{\bullet}, \nu^{\circ}} \in \overline{\mathrm{Z}}_{\pi}^{n}$. Then

$$
\begin{aligned}
& \hat{\phi}_{n}\left(\zeta_{N^{+} M q, N^{-} \ell_{1} \ell_{2} ; \tilde{k}^{\prime}}^{\dagger *} f\right)=\left(\delta_{N^{+} M q, N^{-} \ell_{1}}^{(q, 1)}\right)^{*} \zeta_{N^{+} M, N^{-} \ell_{1}}^{*} f_{\nu\left(\ell_{2}\right) \nu^{\circ}-2 \ell_{2} \nu^{\bullet}, \nu\left(\ell_{2}\right) \nu^{\bullet}-2 \ell_{2} \nu^{\circ}} ; \\
& \hat{\phi}_{n}\left(\zeta_{N^{+} M q, N^{-} \ell_{1} \ell_{2} ; \tilde{k}^{\prime}}^{\ddagger *}\right)=\left(\delta_{N^{+} M q, N^{-} \ell_{1}}^{(q, q)}\right)^{*} \zeta_{N^{+} M, N^{-} \ell_{1}}^{*} f_{\nu\left(\ell_{2}\right) \nu^{\circ}-2 \ell_{2} \nu^{\bullet}, \nu\left(\ell_{2}\right) \nu^{\bullet}-2 \ell_{2} \nu^{\circ},}
\end{aligned}
$$

where $\nu\left(\ell_{2}\right)=\operatorname{tr}\left(\operatorname{Fr}_{\ell_{2}^{2}} ; \mathrm{V}_{\pi}\right)$.

Theorem 4.10 (3) follows from Propositions 3.28 and 3.29, Lemma 4.16, and the following elementary identity

$$
\left(\nu\left(\ell_{2}\right) \nu^{\circ}-2 \ell_{2} \nu^{\bullet}\right)+\epsilon_{\sigma}\left(\ell_{2}\right)\left(\nu\left(\ell_{2}\right) \nu^{\bullet}-2 \ell_{2} \nu^{\circ}\right)=\left(\nu\left(\ell_{2}\right)-2 \epsilon_{\sigma}\left(\ell_{2}\right) \ell_{2}\right)\left(\nu^{\circ}+\epsilon_{\sigma}\left(\ell_{2}\right) \nu^{\bullet}\right) .
$$

4.5.3. Proof of Theorem 4.11. We apply the previous discussion to the case: $R=$ $\mathrm{W}\left(\mathbb{F}_{\ell}^{\mathrm{ac}}\right)\left(k=R \otimes_{\mathbb{Z}} \mathbb{Q}\right.$ and $\left.\tilde{k}=\mathbb{F}_{\ell}^{\mathrm{ac}}\right), X=x_{N^{+} M ; R}, Y=y_{N^{+} M q, N^{-} \ell ; R}$, and $W=$ $\Delta_{N+M, N-\ell ; R}^{\prime}$. Then Theorem 4.11 follows exactly as for Theorem 4.10 (3).

\section{Bounding Selmer groups}

We prove our main theorems in both versions of automorphic representations and of elliptic curves. In §5.1, we develop a Galois-theoretical gadget which is applicable to all finite Galois modules we will consider later. In $\S 5.2$, we prove Theorem 3.17. In $\S 5.3$, we prove Theorems 1.3 and 1.5 by applying the previous theorem.

5.1. Some Galois-theoretical arguments. We first introduce some notation.

Definition 5.1. Let $\mathrm{T}$ be a $\mathbb{Z}_{p}$-module.

(1) For an element $t \in \mathrm{T}$, we define the $p$-divisibility to be

$$
\operatorname{ord}_{p}(t)=\sup \left\{n \in \mathbb{Z}_{\geq 0} \mid t \in p^{n} \mathrm{~T}\right\} .
$$


(2) Suppose that $\mathrm{T}$ has finite exponent and is equipped with a linear action of a group G. Define the reducibility depth of $\mathrm{T}$ to be the least integer $\mathfrak{n}_{\mathrm{T}} \geq 0$ such that

- if $\mathrm{T}^{\prime}$ is a G-stable submodule that is not contained in $p \mathrm{~T}$, then $\mathrm{T}^{\prime}$ contains $p^{\mathfrak{n}_{\mathrm{T}} \mathrm{T}}$;

- for every integer $m \geq 1$, the group $\operatorname{Hom}_{\mathrm{G}}\left(\mathrm{T} / p^{m} \mathrm{~T}, \mathrm{~T} / p^{m} \mathrm{~T}\right) / \mathbb{Z}_{p} \cdot \mathrm{id}$ is annihilated by $p^{\mathfrak{n}_{\mathrm{T}}}$.

Lemma 5.2. Let $\mathrm{T}$ be a stable lattice in a p-adic representation $\mathrm{V}$ of $\Gamma_{\mathbb{Q}}$ that is absolutely irreducible and almost everywhere unramified. Then there exists an integer $\mathfrak{n}(\mathrm{T})$ depending only on $\mathrm{T}$, such that $\overline{\mathrm{T}}^{n}$ has reducibility depth at most $\mathfrak{n}(\mathrm{T})$ for all $n \geq 1$.

Proof. Since $\mathrm{V}$ is irreducible, there are only finitely many stable lattices $\mathrm{T}_{1}, \ldots, \mathrm{T}_{l}$ contained in $\mathrm{T}$ but not containing $p \mathrm{~T}$. Let $\mathfrak{n}^{\prime} \geq 0$ be the smallest integer such that $\mathrm{T}_{i}$ contains $p^{\mathfrak{n}^{\prime}} \mathrm{T}$ for all $i=1, \ldots, l$. For an integer $n \geq 1$, suppose that $\mathrm{T}^{\prime}$ is a G-stable submodule of $\overline{\mathrm{T}}^{n}$ that is not contained in $p \overline{\mathrm{T}}^{n}$. Take $t \in \mathrm{T}^{\prime}$ and lift it to $\tilde{t} \in \mathrm{T}$, the

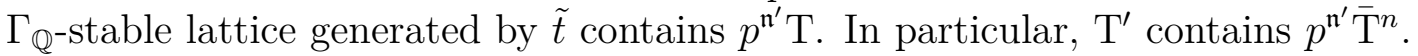

Choose a finite set $\Sigma$ of places of $\mathbb{Q}$ containing $\{\infty, p\}$ such that $\mathrm{V}$ is unramified outside $\Sigma$. For $n \geq 1$, we have a sequence

$$
\operatorname{Hom}_{\Gamma_{\mathbb{Q}}}(\mathrm{T}, \mathrm{T}) / p^{n} \operatorname{Hom}_{\Gamma_{\mathbb{Q}}}(\mathrm{T}, \mathrm{T}) \hookrightarrow \operatorname{Hom}_{\Gamma_{\mathbb{Q}}}\left(\overline{\mathrm{T}}^{n}, \overline{\mathrm{T}}^{n}\right) \rightarrow \operatorname{Ext}_{\Gamma_{\mathbb{Q}, \Sigma}}^{1}(\mathrm{~T}, \mathrm{~T})_{\text {tor }} .
$$

Since $\mathrm{V}$ is absolutely irreducible, the group $\operatorname{Hom}_{\Gamma_{\mathbb{Q}}}(\mathrm{T}, \mathrm{T}) / \mathbb{Z}_{p} \cdot$ id is torsion (and finitely generated). Moreover, the group $\operatorname{Ext}_{\Gamma_{\mathbb{Q}, \Sigma}}^{1}(\mathrm{~T}, \mathrm{~T})_{\text {tor }}$ is also finitely generated. Therefore, there exists some integer $\mathfrak{n}^{\prime \prime} \geq 0$ such that $\operatorname{Hom}_{\Gamma_{\mathbb{Q}}}\left(\overline{\mathrm{T}}^{n}, \overline{\mathrm{T}}^{n}\right) / \mathbb{Z}_{p} \cdot$ id is annihilated by $p^{\mathfrak{n}^{\prime \prime}}$ for all $n \geq 1$. To conclude, take $\mathfrak{n}(\mathrm{T})=\max \left\{\mathfrak{n}^{\prime}, \mathfrak{n}^{\prime \prime}\right\}$.

Let $n \geq 1$ be an integer. Consider a Galois representation $\rho: \Gamma_{\mathbb{Q}} \rightarrow \mathrm{GL}(\mathrm{T})$ where $\mathrm{T}$ is a free $\mathbb{Z} / p^{n}$-module of finite rank. Denote by $\mathrm{G}$ the image of $\rho$, and $L / \mathbb{Q}$ the Galois extension determined by $\mathrm{G}$. We suppose that $\mathrm{G}$ contains a non-trivial scalar element of order coprime to $p$.

Lemma 5.3. We have $\mathrm{H}^{i}(\mathrm{G}, \mathrm{T})=0$ for all $i \geq 0$. The restriction of classes gives an isomorphism:

$$
\operatorname{Res}_{\mathbb{Q}}^{L}: \mathrm{H}^{1}(\mathbb{Q}, \mathrm{T}) \stackrel{\sim}{\rightarrow} \mathrm{H}^{1}(L, \mathrm{~T})^{\mathrm{G}}=\operatorname{Hom}_{\mathrm{G}}\left(\Gamma_{L}^{\mathrm{ab}}, \mathrm{T}\right) .
$$

Proof. The proof is the same as [Gro91, Proposition 9.1].

The above lemma yields a $\mathbb{Z} / p^{n}[\mathrm{G}]$-linear pairing

$$
[,]: \mathrm{H}^{1}(\mathbb{Q}, \mathrm{T}) \times \Gamma_{L}^{\mathrm{ab}} \rightarrow \mathrm{T} .
$$

For each finitely generated $\mathbb{Z} / p^{n}$-submodule $S$ of $\mathrm{H}^{1}(\mathbb{Q}, \mathrm{T})$, denote by $\mathrm{G}_{S}$ the subgroup of $\varrho \in \Gamma_{L}^{\mathrm{ab}}$ such that $[s, \varrho]=0$ for all $s \in S$. Let $L_{S} \subset \mathbb{Q}^{\text {ac }}$ be the subfield fixed by $\mathrm{G}_{S}$. Define a sequence $\mathfrak{f}$ by $\mathfrak{f}(1)=1, \mathfrak{f}(2)=4$ and $\mathfrak{f}(r+1)=2(\mathfrak{f}(r)+1)$. The following lemma generalizes [Gro91, Proposition 9.3].

Lemma 5.4. The induced pairing

$$
[,]: S \times \operatorname{Gal}\left(L_{S} / L\right) \rightarrow \mathrm{T}
$$


yields an injective map $\operatorname{Gal}\left(L_{S} / L\right) \rightarrow \operatorname{Hom}(S, \mathrm{~T})$ of $\mathbb{Z} / p^{n}[\mathrm{G}]$-modules. If $S$ is free of rank $r_{S}$ and $\mathfrak{n}_{\mathrm{T}} \leq n$ is the reducibility depth of $\mathrm{T}$, then the image of $\mathrm{Gal}\left(L_{S} / L\right)$ contains $p^{\mathfrak{f}\left(r_{S}\right) \mathfrak{n}_{\mathrm{T}}} \operatorname{Hom}(S, \mathrm{~T})=\operatorname{Hom}\left(S, p^{\mathfrak{f}\left(r_{S}\right) \mathfrak{n}_{\mathrm{T}} \mathrm{T}}\right)$.

Proof. It is clear that the map $\operatorname{Gal}\left(L_{S} / L\right) \rightarrow \operatorname{Hom}(S, \mathrm{~T})$ is injective and hence we may identify the Galois group $G:=\operatorname{Gal}\left(L_{S} / L\right)$ as a subgroup of $\operatorname{Hom}(S, \mathrm{~T})$. Fix a basis $\left\{s_{1}, \ldots, s_{r}\right\}$ of $S$. We prove by induction on $r$. The case where $r=1$ is immediate, and hence we assume that $r \geq 2$. Put $S_{1}=\bigoplus_{i=1}^{r-1} \mathbb{Z} / p^{n} \cdot s_{i}$ and identify the exact sequence

$$
0 \rightarrow \operatorname{Hom}\left(\mathbb{Z} / p^{n} \cdot s_{r}, \mathrm{~T}\right) \rightarrow \operatorname{Hom}(S, \mathrm{~T}) \rightarrow \operatorname{Hom}\left(S_{1}, \mathrm{~T}\right) \rightarrow 0
$$

with $0 \rightarrow \mathrm{T} \rightarrow \mathrm{T}^{\oplus r} \rightarrow \mathrm{T}^{\oplus r-1} \rightarrow 0$. Denote by $G_{1}$ the image of $G \subset \mathrm{T}^{\oplus r}$ under the map $\mathrm{T}^{\oplus r} \rightarrow \mathrm{T}^{\oplus r-1}$, and $G_{0}=G \cap \mathrm{T}$ its kernel. By induction, the group $G_{1}$ contains $p^{\mathfrak{f}(r-1) \mathfrak{n}_{\mathrm{T}}} \mathrm{T}^{\oplus r-1}$.

We claim that $G_{0}$ contains $p^{(\mathfrak{f}(r-1)+2) \mathfrak{n}_{\mathrm{T}} \mathrm{T}}$. For this, we need to show that $G_{0}$ is not contained in $p^{(\mathfrak{f}(r-1)+1) \mathfrak{n}_{\mathrm{T}}+1} \mathrm{~T}$. Otherwise, the group $G_{0}$ will be annihilated by $p^{n-1-(\mathfrak{f}(r-1)+1) \mathfrak{n}_{\mathrm{T}}}$. Consider the exact sequence

$$
0 \rightarrow \operatorname{Hom}_{\mathrm{G}}\left(G_{1}, \mathrm{~T}\right) \rightarrow \operatorname{Hom}_{\mathrm{G}}(G, \mathrm{~T}) \rightarrow \operatorname{Hom}_{\mathrm{G}}\left(G_{0}, \mathrm{~T}\right),
$$

in which $S$ is naturally contained in $\operatorname{Hom}_{\mathrm{G}}(G, \mathrm{~T})$. Then the element $p^{n-1-(\mathfrak{f}(r-1)+1) \mathfrak{n}_{\mathrm{T}}} s_{r}$ maps to 0 in $\operatorname{Hom}_{\mathrm{G}}\left(G_{0}, \mathrm{~T}\right)$ and hence belongs to $\operatorname{Hom}_{\mathrm{G}}\left(G_{1}, \mathrm{~T}\right)$. For $G_{1}$, we have another exact sequence

$$
0 \rightarrow \operatorname{Hom}_{\mathrm{G}}\left(G_{1} / p^{\mathfrak{f}(r-1) \mathfrak{n}_{\mathrm{T}}} \mathrm{T}^{\oplus r-1}, \mathrm{~T}\right) \rightarrow \operatorname{Hom}_{\mathrm{G}}\left(G_{1}, \mathrm{~T}\right) \rightarrow \operatorname{Hom}_{\mathrm{G}}\left(p^{\mathfrak{f}(r-1) \mathfrak{n}_{\mathrm{T}}} \mathrm{T}^{\oplus r-1}, \mathrm{~T}\right) .
$$

The last term $\operatorname{Hom}_{\mathrm{G}}\left(p^{\mathfrak{f}(r-1) \mathfrak{n}_{\mathrm{T}}} \mathrm{T}^{\oplus r-1}, \mathrm{~T}\right)$, which equals $\operatorname{Hom}_{\mathrm{G}}\left(p^{\mathfrak{f}(r-1) \mathfrak{n}_{\mathrm{T}}} \mathrm{T}^{\oplus r-1}, p^{\mathfrak{f}(r-1) \mathfrak{n}_{\mathrm{T}}} \mathrm{T}\right)$, is isomorphic to $\operatorname{Hom}_{\mathrm{G}}\left(\mathrm{T} / p^{\mathfrak{f}(r-1) \mathfrak{n}_{\mathrm{T}}} \mathrm{T}, \mathrm{T} / p^{\mathfrak{f}(r-1) \mathfrak{n}_{\mathrm{T}} \mathrm{T}}\right)^{\oplus r-1}$, and the natural quotient

$$
\operatorname{Hom}_{\mathrm{G}}\left(\mathrm{T} / p^{\mathfrak{f}(r-1) \mathfrak{n}_{\mathrm{T}}} \mathrm{T}, \mathrm{T} / p^{\mathfrak{f}(r-1) \mathfrak{n}_{\mathrm{T}}} \mathrm{T}\right) / \mathbb{Z} / p^{n-\mathfrak{f}(r-1) \mathfrak{n}_{\mathrm{T}}} \cdot \text { id }
$$

is annihilated by $p^{\mathfrak{n}_{\mathrm{T}}}$. Therefore, there is an element $s \in S_{1}$ such that $p^{n-1-\mathfrak{f}(r-1) \mathfrak{n}_{\mathrm{T}}} s_{r}-s$ maps to 0 in $\operatorname{Hom}_{\mathrm{G}}\left(p^{\mathfrak{f}(r-1) \mathfrak{n}_{\mathrm{T}}} \mathrm{T}^{\oplus r-1}, \mathrm{~T}\right)$. In other words, the element $p^{n-1-\mathfrak{f}(r-1) \mathfrak{n}_{\mathrm{T}}} s_{r}-s$ belongs to $\operatorname{Hom}_{G}\left(G_{1} / p^{\mathfrak{f}(r-1) \mathfrak{n}_{\mathrm{T}}} \mathrm{T}^{\oplus r-1}, \mathrm{~T}\right)$, a group annihilated by $p^{\mathfrak{f}(r-1) \mathfrak{n}_{\mathrm{T}}}$. This implies that $p^{n-1} s_{r}-p^{\mathrm{f}(r-1) \mathfrak{n}_{\mathrm{T}}} s=0$ in $\operatorname{Hom}_{\mathrm{G}}(G, \mathrm{~T})$, which contradicts the fact that $S$ is free.

Now consider the exact sequence $0 \rightarrow G_{0} \rightarrow G \rightarrow G_{1} \rightarrow 0$. For every $t \in \mathrm{T}^{\oplus r}$, the image of $p^{\mathfrak{f}(r-1) \mathfrak{n}_{\mathrm{T}}} t$ in $\mathrm{T}^{\oplus r-1}$ is contained in $G_{1}$ by the induction assumption. Pick up an element $g \in G$ such that $g-p^{\mathfrak{f}(r-1) \mathfrak{n}_{\mathrm{T}}} t \in \mathrm{T}=\operatorname{ker}\left[\mathrm{T}^{\oplus r} \rightarrow \mathrm{T}^{\oplus r-1}\right]$. By the previous claim, $p^{(\mathfrak{f}(r-1)+2) \mathfrak{n}_{\mathrm{T}}}\left(g-p^{\mathfrak{f}(r-1) \mathfrak{n}_{\mathrm{T}}} t\right)$ belongs to $G_{0}$, which implies that $p^{(\mathfrak{f}(r-1)+2) \mathfrak{n}_{\mathrm{T}}} p^{\mathfrak{f}(r-1) \mathfrak{n}_{\mathrm{T}}} t=$ $p^{\mathrm{f}(r) \mathfrak{n}_{\mathrm{T}}} t$ belongs to $G$.

Notation 5.5. For each place $w$ of $L$, we denote by $\ell(w)$ the prime underlying $w$. If $\ell(w)$ is unramified in $L_{S}$, then the Frobenius substitution in $\mathrm{H}:=\operatorname{Gal}\left(L_{S} / L\right)$ of an arbitrary place of $L_{S}$ above $w$ is well-defined since $\mathrm{H}$ is abelian. It will be denoted by $\Psi_{w}$. 
Take a non-trivial finite free $\mathbb{Z} / p^{n}$-submodule $S$ of $\mathrm{H}^{1}(\mathbb{Q}, \mathrm{T})$. Let $\varrho$ be an element of $\mathrm{G}$ of order $l$ coprime to $p$. Consider the following diagram of field extension

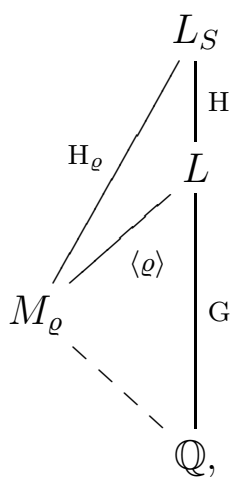

in which $M_{\varrho}$ is the subfield fixed by the cyclic subgroup $\langle\varrho\rangle \subset \mathrm{G}$ generated by $\varrho$, and $\mathrm{H}_{\varrho}=\operatorname{Gal}\left(L_{S} / M\right)$. In particular, we have an isomorphism $\mathrm{H}_{\varrho} \simeq \mathrm{H} \rtimes\langle\varrho\rangle \subset$ $\operatorname{Hom}(S, \mathrm{~T}) \rtimes\langle\varrho\rangle$ since $\langle\varrho\rangle$ is of order coprime to $p$.

Definition 5.6. Let $\Sigma$ be a finite set of primes containing those ramified in $L_{S}$. We say that a finite place $w$ of $L$ is $(\Sigma, \varrho)$-admissible if its underlying prime $\ell(w)$ does not belong to $\Sigma$, and its Frobenius substitution in $\operatorname{Gal}(L / \mathbb{Q})=\mathrm{G}$, which is then welldefined, is $\varrho$.

Let $w$ be a $(\Sigma, \varrho)$-admissible place of $L$. Then $\operatorname{loc}_{\ell(w)}(s)$ belongs to $\mathrm{H}_{\mathrm{unr}}^{1}\left(\mathbb{Q}_{\ell(w)}, \mathrm{T}\right)$, where the latter equals $\mathrm{H}^{1}\left(\mathbb{F}_{\ell(w)}, \mathrm{T}\right)$ which is contained in $\operatorname{Hom}\left(\Gamma_{\ell(w)} / \mathrm{I}_{\ell(w)}, \mathrm{T}\right) \simeq \mathrm{T}$, where the last isomorphism is induced by taking the image of $\operatorname{Fr}_{\ell(w)}$.

Lemma 5.7. Let $w$ be a $(\Sigma, \varrho)$-admissible place of $L$. Then for $s \in S$, we have

$$
\left[s, \Psi_{w}\right]=\operatorname{loc}_{\ell(w)}(s)\left(\Psi_{w}\right)
$$

as an equality in $\mathrm{T}$.

Proof. Consider the following composite map

$$
S \rightarrow \mathrm{H}^{1}(\mathbb{Q}, \mathrm{T}) \stackrel{\operatorname{Res}_{\mathbb{Q}}^{L}}{\longrightarrow} \mathrm{H}^{1}(L, \mathrm{~T}) \stackrel{\operatorname{loc}_{w}}{\longrightarrow} \mathrm{H}^{1}\left(L_{w}, \mathrm{~T}\right)
$$

whose image is contained in $\mathrm{H}_{\mathrm{unr}}^{1}\left(L_{w}, \mathrm{~T}\right)$. By definition, $\left[, \Psi_{w}\right]$ is simply the composition of the above map with the following one $\mathrm{H}_{\mathrm{unr}}^{1}\left(L_{w}, \mathrm{~T}\right) \rightarrow \mathrm{T}$ obtained by evaluating at $\Psi_{w}$.

The lemma follows since we have the following commutative diagram

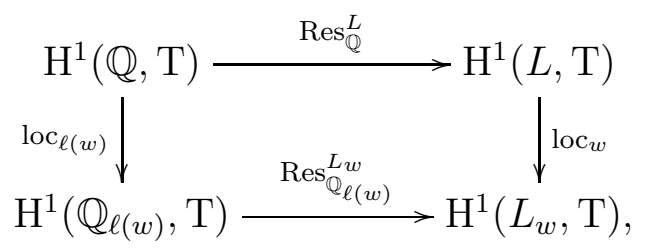

in which the bottom arrow is injective since $\operatorname{Gal}\left(L_{w} / \mathbb{Q}_{\ell(w)}\right) \simeq\langle\varrho\rangle$ has order coprime to $p$. 
Lemma 5.8. The subset of $\Psi_{w} \in \mathrm{H} \subset \operatorname{Hom}(S, \mathrm{~T})$ for $(\Sigma, \varrho)$-admissible places $w$ is exactly $\mathrm{H} \cap \operatorname{Hom}\left(S, \mathrm{~T}^{\langle\varrho\rangle}\right)$.

Proof. By Galois theory, an element $\rho$ in $\mathrm{H}$ is of the form $\Psi_{w}$ if and only if $\rho=\widetilde{h}^{l}$ with $\widetilde{h} \in \mathrm{H}_{\varrho}$ which maps to $\varrho$ under the quotient homomorphism $\mathrm{H}_{\varrho} \rightarrow\langle\varrho\rangle$. Suppose that $\widetilde{h}=(h, \varrho)$ with $h: S \rightarrow$ T in H. Then $\widetilde{h}^{l}=\left(h^{\prime}, 1\right)$ where $h^{\prime}(s)=\left(\varrho^{l-1}+\cdots+\varrho+1\right) h(s) \in$ $\mathrm{T}^{\langle\varrho\rangle}$. Therefore, the element $\rho$ belongs to $\operatorname{Hom}\left(S, \mathrm{~T}^{\langle\varrho\rangle}\right)$. The other inclusion is trivial since $l$ is invertible in $\mathbb{Z} / p^{n}$.

5.2. Proof of Theorem 3.17. Put $\mathrm{T}_{\sigma \times \breve{\pi}}=\mathrm{T}_{\sigma, \pi} \cap \mathrm{V}_{\sigma \times \breve{\pi}}$ and $\mathrm{T}_{\sigma \otimes \breve{\eta}}=\mathrm{T}_{\sigma, \pi} \cap \mathrm{V}_{\sigma \otimes \breve{\eta}}$.

Lemma 5.9. For subscripts $?=\sigma, \pi ; \sigma \times \breve{\pi} ; \sigma \otimes \breve{\eta}$, if $\operatorname{dim}_{\mathbb{Q}_{p}} \mathrm{H}_{f}^{1}\left(\mathbb{Q}, \mathrm{V}_{?}\right)=r$, then there is a free $\mathbb{Z} / p^{n}$-submodule of $\mathrm{H}_{f}^{1}\left(\mathbb{Q}, \overline{\mathrm{T}}_{?}^{n}\right)$ (see Definition 3.1) of rank $r$.

Proof. Recall that we define $\mathrm{H}_{f}^{1}\left(\mathbb{Q}, \mathrm{T}_{?}\right) \subset \mathrm{H}^{1}\left(\mathbb{Q}, \mathrm{T}_{\text {? }}\right)$ to be the inverse image of $\mathrm{H}_{f}^{1}\left(\mathbb{Q}, \mathrm{V}_{\text {? }}\right)$ under the natural map $\mathrm{H}^{1}\left(\mathbb{Q}, \mathrm{T}_{?}\right) \rightarrow \mathrm{H}^{1}\left(\mathbb{Q}, \mathrm{V}_{?}\right)$, and $\mathrm{H}_{f}^{1}\left(\mathbb{Q}, \overline{\mathrm{T}}_{?}^{n}\right)$ to be the image of $\mathrm{H}_{f}^{1}\left(\mathbb{Q}, \mathrm{T}_{\text {? }}\right)$ the natural map $\mathrm{H}^{1}\left(\mathbb{Q}, \mathrm{T}_{\text {? }}\right) \rightarrow \mathrm{H}^{1}\left(\mathbb{Q}, \overline{\mathrm{T}}_{?}^{n}\right)$.

Suppose that $\operatorname{dim}_{\mathbb{Q}_{p}} \mathrm{H}_{f}^{1}\left(\mathbb{Q}, \mathrm{V}_{\text {? }}\right)=r$. Since $\mathrm{H}_{f}^{1}\left(\mathbb{Q}, \mathrm{T}_{\text {? }}\right)$ is finitely generated, we may choose $s_{1}, \ldots, s_{r} \in \mathrm{H}_{f}^{1}\left(\mathbb{Q}, \mathrm{T}_{\text {? }}\right)$ such that they generate a saturated free $\mathbb{Z}_{p}$-submodule. In particular, the image of $s_{1}, \ldots, s_{r}$ under the natural map

$$
\mathrm{H}_{f}^{1}\left(\mathbb{Q}, \mathrm{T}_{?}\right) \rightarrow \mathrm{H}_{f}^{1}\left(\mathbb{Q}, \mathrm{T}_{?}\right) / p^{n} \mathrm{H}_{f}^{1}\left(\mathbb{Q}, \mathrm{T}_{?}\right) \hookrightarrow \mathrm{H}^{1}\left(\mathbb{Q}, \mathrm{T}_{?}\right) / p^{n} \mathrm{H}^{1}\left(\mathbb{Q}, \mathrm{T}_{?}\right) \hookrightarrow \mathrm{H}^{1}\left(\mathbb{Q}, \overline{\mathrm{T}}_{?}^{n}\right)
$$

span a free $\mathbb{Z} / p^{n}$-submodule of rank $r$, which is contained in $\mathrm{H}_{f}^{1}\left(\mathbb{Q}, \overline{\mathrm{T}}_{?}^{n}\right)$ by definition.

The following discussion will be under all of Assumption 3.8, until the end of this section.

Lemma 5.10. Let $n \geq 1$ be an integer. We have that

(1) If $\wp\left(N^{-}\right)$is even, then the class $\bar{\Delta}_{\sigma, \pi}^{n}$ belongs to $\mathrm{H}_{f}^{1}\left(\mathbb{Q}, \overline{\mathrm{T}}_{\sigma, \pi}^{n}\right)$.

(2) If $\wp\left(N^{-}\right)$is even and $\ell_{1}, \ell_{2}$ are two distinct strongly $n$-admissible primes, then

$$
\operatorname{loc}_{v}\left(\bar{\Delta}_{\sigma, \pi \mid \ell_{1}, \ell_{2}}^{n}\right) \in \mathrm{H}_{\mathrm{unr}}^{1}\left(\mathbb{Q}_{v}, \overline{\mathrm{T}}_{\sigma, \pi}^{n}\right)
$$

for all primes $v \nmid p q N^{-} \mathcal{D}_{N^{+}}{ }_{M} \ell_{1} \ell_{2}$, and

$$
\operatorname{loc}_{p}\left(\bar{\Delta}_{\sigma, \pi \mid \ell_{1}, \ell_{2}}^{n}\right) \in \mathrm{H}_{f}^{1}\left(\mathbb{Q}_{p}, \overline{\mathrm{T}}_{\sigma, \pi}^{n}\right) .
$$

(3) If $\wp\left(N^{-}\right)$is odd and $\ell$ is a strongly $n$-admissible prime, then

$$
\operatorname{loc}_{v}\left(\bar{\Delta}_{\sigma, \pi \mid \ell}^{n}\right) \in \mathrm{H}_{\mathrm{unr}}^{1}\left(\mathbb{Q}_{v}, \overline{\mathrm{T}}_{\sigma, \pi}^{n}\right)
$$

for all primes $v \nmid p q N^{-} \mathcal{D}_{N^{+} M} \ell$, and

$$
\operatorname{loc}_{p}\left(\bar{\Delta}_{\sigma, \pi \mid \ell}^{n}\right) \in \mathrm{H}_{f}^{1}\left(\mathbb{Q}_{p}, \overline{\mathrm{T}}_{\sigma, \pi}^{n}\right) .
$$

Proof. Part (1) follows by Proposition 3.15, Definition 3.1, and the construction of $\bar{\Delta}_{\sigma, \pi}^{n}$. For $(2)$, since the $p$-adic representation

$$
\mathrm{H}_{\text {ét }}^{1}\left(Y_{N+M, N-\ell_{1} \ell_{2} ; \mathbb{Q}_{v}^{\text {ac }}}, \mathbb{Q}_{p}(1)\right) \otimes \mathrm{H}_{\text {cusp }}^{2}\left(X_{N+M ; \mathbb{Q}_{v}^{\text {ac }}}, \mathbb{Q}_{p}(1)\right)
$$


is unramified at all primes $v \nmid p q N^{-} \mathcal{D}_{N^{+} M} \ell_{1} \ell_{2}$, we have $\operatorname{loc}_{v}\left(\bar{\Delta}_{\sigma, \pi \mid \ell_{1}, \ell_{2}}^{n}\right) \in \mathrm{H}_{\text {unr }}^{1}\left(\mathbb{Q}_{v}, \overline{\mathrm{T}}_{\sigma, \pi}^{n}\right)$ by Lemma 3.4 and the construction of $\bar{\Delta}_{\sigma, \pi \mid \ell_{1}, \ell_{2}}^{n}$. For the localization at $p$, the argument is the same as the last part of the proof of Proposition 3.15. Part (3) is the same as (2).

\section{Notation 5.11.}

(1) If $\wp\left(N^{-}\right)$is even, denote by $n_{\text {div }}$ the $p$-divisibility $\operatorname{ord}_{p}\left(\bar{\Delta}_{\sigma, \pi}^{\infty}\right)$ of $\bar{\Delta}_{\sigma, \pi}^{\infty}$. If $\wp\left(N^{-}\right)$ is odd, denote by $n_{\text {div }}$ the $p$-divisibility of (3.4) as a non-zero number in $\mathbb{Z}_{p}$. Then $n_{\text {div }} \geq 0$ is an integer.

(2) By Remark 3.32 (1) and (R4), the density of strongly $(n,-)$-admissible primes among all $n$-admissible primes is strictly positive and independent of $n$. We denote it by $\mathfrak{d}$. Let $n_{\text {den }} \geq 0$ be the smallest integer such that

$$
p^{n_{\mathrm{den}}+1}>\mathfrak{d}^{-1} \text {. }
$$

For our convenience, we record the following corollary of Theorems 4.10 and 4.11.

Proposition 5.12. Let $n \geq 1$ be an integer.

(1) Suppose that $\wp\left(N^{-}\right)$is even. Let $\ell_{1}, \ell_{2}$ be two distinct strongly $n$-admissible primes. Then

$$
\operatorname{ord}_{p}\left(\operatorname{loc}_{\ell_{1}}\left(\bar{\Delta}_{\sigma, \pi}^{n}\right)\right)=\operatorname{ord}_{p}\left(\partial_{\ell_{2}} \operatorname{loc}_{\ell_{2}}\left(\bar{\Delta}_{\sigma, \pi \mid \ell_{1}, \ell_{2}}^{n}\right)\right),
$$

where

$$
\begin{aligned}
\operatorname{loc}_{\ell_{1}}\left(\bar{\Delta}_{\sigma, \pi}^{n}\right) & \in \mathrm{H}_{\text {unr }}^{1}\left(\mathbb{Q}_{\ell_{1}}, \overline{\mathrm{T}}_{\sigma, \pi}^{n}\right) \simeq \mathbb{Z} / p^{n}, \\
\partial_{\ell_{2}} \operatorname{loc}_{\ell_{2}}\left(\bar{\Delta}_{\sigma, \pi \mid \ell_{1}, \ell_{2}}^{n}\right) & \in \mathrm{H}_{\text {sing }}^{1}\left(\mathbb{Q}_{\ell_{1}}, \overline{\mathrm{T}}_{\sigma, \pi}^{n}\right) \simeq \mathbb{Z} / p^{n}
\end{aligned}
$$

by Proposition 4.2.

(2) Suppose that $\wp\left(N^{-}\right)$is odd. Let $\ell$ be a strongly $n$-admissible prime. Then

$$
\operatorname{ord}_{p}\left(\partial_{\ell} \operatorname{loc}_{\ell}\left(\bar{\Delta}_{\sigma, \pi \mid \ell}^{n}\right)\right)=n_{\text {div }}
$$

where $n_{\text {div }}$ is the p-divisibility of (3.4) as in Notation 5.11.

5.2.1. Proof under Assumption 3.8 (R6a). In this case, the representation $\left(\rho_{\sigma, \pi}, \mathrm{V}_{\sigma, \pi}\right)$ is absolutely irreducible. Denote by $n_{\text {red }}=\mathfrak{n}\left(\mathrm{T}_{\sigma, \pi}\right)$ the integer in Lemma 5.2.

First, consider the case where $\wp\left(N^{-}\right)$is even. We prove by contradiction, and hence assume that $\operatorname{dim}_{\mathbb{Q}_{p}} \mathrm{H}_{f}^{1}\left(\mathbb{Q}, \mathrm{V}_{\sigma, \pi}\right) \geq 2$. Let $n$ be a sufficiently large integer which will be determined later. By Lemma 5.9, we may take a free $\mathbb{Z} / p^{n}$-submodule $S$ of $\mathrm{H}_{f}^{1}\left(\mathbb{Q}, \overline{\mathrm{T}}_{\sigma, \pi}^{n}\right)$ of rank 2. Moreover, we may assume that $S$ has a basis $\left\{s_{1}, s_{2}\right\}$ such that $\bar{\Delta}_{\sigma, \pi}^{n}=p^{n_{\text {div }}} s_{1}$.

By (R4), there exists a strongly $n$-admissible prime. We denote the image of its Frobenius substitution under $\bar{\rho}_{\sigma, \pi}^{n}$ by $\varrho \in \mathrm{G}$. By raising a sufficiently large $p$-power, we may assume that $\varrho$ has order $l$ coprime to $p$. We would like to apply the discussion in $\$ 5.1$ to $\mathrm{T}=\overline{\mathrm{T}}_{\sigma, \pi}^{n}$ and $\varrho$ as above. Assumption 3.8 (R6a) implies that $\mathrm{G}$ contains a non-trivial scalar element of order coprime to $p$. Let $\Sigma$ be the set of primes dividing $2 p q N^{-} \mathcal{D}_{N^{+} M}$ and those ramified in $L_{S}$.

Lemma 5.13. If $w$ is a $(\Sigma, \varrho)$-admissible place of $L$, then its underlying prime $\ell(w)$ is strongly $n$-admissible. 
Proof. Since the kernel of the homomorphism $\mathrm{GL}\left(\overline{\mathrm{T}}_{\sigma}^{n}\right) \times \mathrm{O}\left({ }^{\sharp} \overline{\mathrm{T}}_{\pi}^{n}\right) \stackrel{\otimes}{\rightarrow} \mathrm{GL}\left(\overline{\mathrm{T}}_{\sigma, \pi}^{n}\right)$ consists of $\left(\mu^{-1}, \mu\right)$ for some $\mu \in\left(\mathbb{Z} / p^{n}\right)^{\times}$of order 4 , the lemma follows by Definition 3.31 (S2) and Remark 3.32.

By Lemmas 5.4 and 5.8, we may choose two $(\Sigma, \varrho)$-admissible places $w_{1}, w_{2}$ of $L$ such that

$$
\Psi_{w_{1}}\left(s_{2}\right)=0, \quad \Psi_{w_{1}}\left(s_{1}\right)=t_{1}, \quad \Psi_{w_{2}}\left(s_{2}\right)=t_{2}
$$

with $t_{1}, t_{2} \in\left(\overline{\mathrm{T}}_{\sigma, \pi}^{n}\right)^{\langle\varrho\rangle}$ such that $\operatorname{ord}_{p}\left(t_{1}\right)=\operatorname{ord}_{p}\left(t_{2}\right)=4 n_{\text {red }}$, and $\ell_{1}:=\ell\left(w_{1}\right), \ell_{2}:=\ell\left(w_{2}\right)$ are distinct. By Lemma 5.7, we have

$$
\operatorname{loc}_{\ell_{1}}\left(s_{2}\right)=0, \quad \operatorname{loc}_{\ell_{1}}\left(\bar{\Delta}_{\sigma, \pi}^{n}\right)\left(\operatorname{Fr}_{\ell_{1}}\right)=p^{n_{\mathrm{div}}} t_{1} / l .
$$

By Proposition $5.12(1)$, the $p$-divisibility of $\partial_{\ell_{2}} \operatorname{loc}_{\ell_{2}}\left(\bar{\Delta}_{\sigma, \pi \mid \ell_{1}, \ell_{2}}^{n}\right)$ in $\mathrm{H}_{\text {sing }}^{1}\left(\mathbb{Q}_{\ell_{2}}, \overline{\mathrm{T}}_{\sigma, \pi}^{n}\right) \simeq$ $\mathbb{Z} / p^{n}$ is $n_{\text {div }}+4 n_{\text {red }}$.

By Lemmas 5.10, 3.4, 4.3 (2) and 4.8, we have

$$
\left\langle s_{2}, \bar{\Delta}_{\sigma, \pi \mid \ell_{1}, \ell_{2}}^{n}\right\rangle_{v}=0,
$$

for all primes $v \nmid q N^{-} \mathcal{D}_{N^{+}}{ }_{M} \ell_{1} \ell_{2}$. We also have

$$
\left\langle s_{2}, \bar{\Delta}_{\sigma, \pi \mid \ell_{1}, \ell_{2}}^{n}\right\rangle_{\ell_{1}}=0
$$

since $\operatorname{loc}_{\ell_{1}}\left(s_{2}\right)=0$. For every prime $v$ divides $q N^{-} \mathcal{D}_{N^{+} M}$, we have

$$
p^{n_{\mathrm{bad}}}\left\langle s_{2}, \bar{\Delta}_{\sigma, \pi \mid \ell_{1}, \ell_{2}}^{n}\right\rangle_{v}=0
$$

where $n_{\text {bad }}$ is introduced in Notation 4.4. By our choice of $w_{2}$ and the fact that $\langle,\rangle_{\ell_{2}}$ induces a perfect pairing between $\mathrm{H}_{\mathrm{unr}}^{1}\left(\mathbb{Q}_{\ell_{2}}, \overline{\mathrm{T}}_{\sigma, \pi}^{n}\right)$ and $\mathrm{H}_{\text {sing }}^{1}\left(\mathbb{Q}_{\ell_{2}}, \overline{\mathrm{T}}_{\sigma, \pi}^{n}\right)$, we have $\operatorname{ord}_{p}\left(\left\langle s_{2}, \bar{\Delta}_{\sigma, \pi \mid \ell_{1}, \ell_{2}}^{n}\right\rangle \ell_{2}\right)=n_{\mathrm{div}}+8 n_{\mathrm{red}}$. If $n>n_{\mathrm{div}}+n_{\text {bad }}+8 n_{\text {red }}$, then together with (5.1), (5.2), (5.3), we have $\sum_{v}\left\langle s_{2}, \bar{\Delta}_{\sigma, \pi \mid \ell_{1}, \ell_{2}}^{n}\right\rangle_{v} \neq 0$, which contradicts Lemma 4.1. Therefore, Theorem 3.17 (2) follows under Assumption 3.8 (R6a).

Next, consider the case where $\wp\left(N^{-}\right)$is odd. We prove by contradiction, and hence assume that $\operatorname{dim}_{\mathbb{Q}_{p}} \mathrm{H}_{f}^{1}\left(\mathbb{Q}, \mathrm{V}_{\sigma, \pi}\right) \geq 1$. Let $n$ be a sufficiently large integer which will be determined later. By Lemma 5.9, we may take a free $\mathbb{Z} / p^{n}$-submodule $S$ of $\mathrm{H}_{f}^{1}\left(\mathbb{Q}, \overline{\mathrm{T}}_{\sigma, \pi}^{n}\right)$ of rank 1 with a basis $\{s\}$.

By (R4), there exists a strongly $n$-admissible prime. We denote its image under $\bar{\rho}_{\sigma, \pi}^{n}$ by $\varrho \in \mathrm{G}$. By raising a sufficiently large $p$-power, we may assume that $\varrho$ has order $l$ coprime to $p$. We would like to apply the discussion in $\S 5.1$ to $\mathrm{T}=\overline{\mathrm{T}}_{\sigma, \pi}^{n}$ and $\varrho$ as above. Assumption 3.8 (R6a) implies that $\mathrm{G}$ contains a non-trivial scalar element of order coprime to $p$. Let $\Sigma$ be the set of primes dividing $2 p q N^{-} \mathcal{D}_{N^{+} M}$ and those ramified in $L_{S}$. If $w$ is a $(\Sigma, \varrho)$-admissible place of $L$, then its underlying prime $\ell(w)$ is strongly $n$-admissible.

By Lemma 5.8, we may choose a $(\Sigma, \varrho)$-admissible place $w$ of $L$ such that $\operatorname{ord}_{p}\left(\Phi_{w}(s)\right)=n_{\text {red }}$. By Proposition $5.12(2)$, the $p$-divisibility of $\partial_{\ell} \operatorname{loc}_{\ell}\left(\bar{\Delta}_{\sigma, \pi \mid \ell}^{n}\right)$ in $\mathrm{H}_{\text {sing }}^{1}\left(\mathbb{Q}_{\ell}, \overline{\mathrm{T}}_{\sigma, \pi}^{n}\right) \simeq \mathbb{Z} / p^{n}$ is $n_{\text {div }}$. We will obtain a contradiction when $n>n_{\text {div }}+n_{\text {red }}+n_{\text {bad }}$. Therefore, Theorem 3.17 (1) follows under Assumption 3.8 (R6a). 
5.2.2. Proof under Assumption 3.8 (R6b). In this case, we have $\mathrm{T}_{\sigma, \pi}=\mathrm{T}_{\sigma \times \breve{\pi}} \oplus \mathrm{T}_{\sigma \otimes \breve{\eta}}$. Denote by $\bar{\Delta}_{\sigma \times \breve{\pi}}^{n}\left(\operatorname{resp} . \bar{\Delta}_{\sigma \otimes \breve{\eta}}^{n}\right)$ the projection of $\bar{\Delta}_{\sigma, \pi}^{n}$ to $\mathrm{H}^{1}\left(\mathbb{Q}, \overline{\mathrm{T}}_{\sigma \times \breve{\pi}}^{n}\right)\left(\operatorname{resp} . \mathrm{H}^{1}\left(\mathbb{Q}, \overline{\mathrm{T}}_{\sigma \otimes \breve{\eta}}^{n}\right)\right)$ for $1 \leq n \leq \infty$. Since the representation $\left(\rho_{\sigma \times \breve{\pi}}, \mathrm{V}_{\sigma \times \breve{\pi}}\right)$ is absolutely irreducible, we have the integer $n_{\text {red }}:=\mathfrak{n}\left(\mathrm{T}_{\sigma \times \breve{\pi}}\right)$ from Lemma 5.2. Moreover, we may take $\mathfrak{n}\left(\mathrm{T}_{\sigma \otimes \breve{\eta}}\right)=0$ by Assumption 3.8 (R2).

In what follows, we only prove for Theorem 3.17 (2), since the proof for Theorem 3.17 (1) is very similar and easier, as we can see from the proof under Assumption 3.8 (R6a). The proof for Theorem 3.17 (1) will be left to the reader.

We divide the proof of Theorem 3.17 (2) under Assumption 3.8 (R6b) into the following four lemmas.

Lemma 5.14. If $\bar{\Delta}_{\sigma \times \breve{\pi}}^{\infty}$ is non-torsion, then $\operatorname{dim}_{\mathbb{Q}_{p}} \mathrm{H}^{1}\left(\mathbb{Q}, \mathrm{V}_{\sigma \times \breve{\pi}}\right)=1$.

For this statement, we do not need to consider $\mathfrak{d}$ and $n_{\text {den }}$.

Proof. We prove by contradiction, and hence assume that $\operatorname{dim}_{\mathbb{Q}_{p}} \mathrm{H}_{f}^{1}\left(\mathbb{Q}, \mathrm{V}_{\sigma \times \breve{\pi}}\right) \geq 2$. Let $n$ be a sufficiently large integer which will be determined later. By Lemma 5.9, we may take a free $\mathbb{Z} / p^{n}$-submodule $S$ of $\mathrm{H}_{f}^{1}\left(\mathbb{Q}, \overline{\mathrm{T}}_{\sigma \times \breve{\pi}}^{n}\right)$ of rank 2. Moreover, we may assume that $S$ has a basis $\left\{s_{1}, s_{2}\right\}$ such that $\bar{\Delta}_{\sigma \times \breve{\pi}}^{n}=p^{n_{\text {div }}} s_{1}$.

By (R4), there exists a strongly $(n,+)$-admissible prime. We denote its image under $\bar{\rho}_{\sigma \times \breve{\pi}}^{n}$ by $\varrho \in \mathrm{G}$. By raising a sufficiently large $p$-power, we may assume that $\varrho$ has order coprime to $p$. We apply the discussion in $\$ 5.1$ to $\mathrm{T}=\overline{\mathrm{T}}_{\sigma \times \bar{\pi}}^{n}$ and $\varrho$ as above. Assumption 3.8 (R6b) implies that $\mathrm{G}$ contains a non-trivial scalar element of order coprime to $p$. Let $\Sigma$ be the set of primes dividing $2 p q N^{-} \mathcal{D}_{N^{+} M}$ and those ramified in $L_{S}$. Note that $\mathrm{T}^{\langle\varrho\rangle}$ is free of rank 1 . Let $w$ be a $(\Sigma, \varrho)$-admissible place of $L$. We claim that its underlying prime $\ell(w)$ is strongly $(n,+)$-admissible. This follows from the observation that the kernel of the homomorphism $\operatorname{GL}\left(\overline{\mathrm{T}}_{\sigma}^{n}\right) \times \mathrm{GL}\left(\overline{\mathrm{T}}_{\breve{\pi}}^{n}(-1)\right) \stackrel{\otimes}{\rightarrow} \mathrm{GL}\left(\overline{\mathrm{T}}_{\sigma \times \breve{\pi}}^{n}\right)$ consists of $\left(\mu^{-1}, \mu\right)$ for some $\mu \in\left(\mathbb{Z} / p^{n}\right)^{\times}$of order 3. However by Remark $3.32(2)$, the element $\mu \bar{\rho}_{\breve{\pi}}^{n}(-1)\left(\operatorname{Fr}_{\ell(w)}\right)$ belongs to the image of $\bar{\rho}_{\breve{\pi}}^{n}(-1)$ (here, $\rho_{\breve{\pi}}$ is the underlying representation of $\mathrm{T}_{\breve{\pi}}$ ) only when $\mu=1$.

The rest follows in the same way as the case under (R6a).

Lemma 5.15. If $\bar{\Delta}_{\sigma \times \breve{\pi}}^{\infty}$ is non-torsion, then $\operatorname{dim}_{\mathbb{Q}_{p}} \mathrm{H}^{1}\left(\mathbb{Q}, \mathrm{V}_{\sigma \otimes \breve{\eta}}\right)=0$.

Proof. We prove by contradiction, and hence assume that $\operatorname{dim}_{\mathbb{Q}_{p}} \mathrm{H}_{f}^{1}\left(\mathbb{Q}, \mathrm{V}_{\sigma \otimes \breve{\eta}}\right) \geq 1$. Let $n$ be a sufficiently large integer which will be determined later. By Lemma 5.9, we may take a free $\mathbb{Z} / p^{n}$-submodule $S_{1}\left(\operatorname{resp} . S_{2}\right)$ of $\mathrm{H}_{f}^{1}\left(\mathbb{Q}, \overline{\mathrm{T}}_{\sigma \times \breve{\pi}}^{n}\right)\left(\operatorname{resp} . \mathrm{H}_{f}^{1}\left(\mathbb{Q}, \overline{\mathrm{T}}_{\sigma \otimes \grave{\eta}}^{n}\right)\right)$ of rank 1 with basis $\left\{s_{1}\right\}$ (resp. $\left\{s_{2}\right\}$ ). Moreover, we may assume that $\bar{\Delta}_{\sigma \times \breve{\pi}}^{n}=p^{n_{\text {div }}} s_{1}$. A similar argument from the above lemma provides us with a strongly $(n,+)$-admissible prime $\ell_{1}$ such that $\operatorname{ord}_{p}\left(\operatorname{loc}_{\ell_{1}}\left(\bar{\Delta}_{\sigma, \pi}^{n}\right)\left(\operatorname{Fr}_{\ell_{1}}\right)\right)=n_{\text {div }}+n_{\text {red }}$.

We apply the discussion in $\S 5.1$ to $\mathrm{T}=\overline{\mathrm{T}}_{\sigma \otimes \breve{\eta}}^{n}$. Note that (R3) implies that G contains a non-trivial scalar element of order coprime to $p$. Let $\Sigma$ be the set of primes dividing $2 p q N^{-} \mathcal{D}_{N^{+} M}$ and those ramified in $L_{S_{2}}$. If we put $\varrho_{1}=\bar{\rho}_{\sigma \otimes \breve{\eta}}^{n}\left(\mathrm{Fr}_{\ell_{1}}\right) \in \mathrm{G}$, then it has order coprime to $p$ and $\left(\overline{\mathrm{T}}_{\sigma \otimes \breve{\eta}}^{n}\right)^{\left\langle\varrho_{1}\right\rangle}=0$. This implies that $\operatorname{loc}_{\ell_{1}}\left(s_{2}\right)=0$.

Consider the composite homomorphism

$$
\chi: \Gamma_{\mathbb{Q}} \stackrel{\chi_{1}}{\rightarrow} \operatorname{Gal}\left(L_{S_{2}} / \mathbb{Q}\right) \times \operatorname{Gal}(\breve{F} / \mathbb{Q}) \rightarrow \mathrm{G} \times \operatorname{Gal}(\breve{F} / \mathbb{Q}),
$$


in which the second factor of $\chi_{1}$ is the natural projection. Since $\breve{F}$ is unramified outside $N, \chi$ is surjective. Since the kernel of the second homomorphism is a $p$-group (and $p$ is odd) and the image of $\chi_{1}$ has the index at most 2 , the homomorphism $\chi_{1}$ is surjective as well.

Let $\ell$ be an $n$-admissible prime that is unramified in $L_{S_{2}}$ such that $\epsilon_{\sigma}(\ell)=\breve{\eta}\left(\mathbb{F}_{\ell}\right)$. Take an arbitrary place $w$ of $L$ above $\ell$ and denote its Frobenius substitution in $G$ by $\varrho$. The $p$-divisibility $\operatorname{ord}_{p}\left(\Psi_{w}\left(s_{2}\right)\right)$ of $\Psi_{w}\left(s_{2}\right) \in\left(\overline{\mathrm{T}}_{\sigma \otimes \breve{\eta}}^{n}\right)^{\langle\varrho\rangle} \simeq \mathbb{Z} / p^{n}$ is independent of the choice of $w$. By the Chebotarev Density Theorem and the fact that $\chi_{1}$ is surjective, the density of those $\ell$ among all $n$-admissible primes satisfying $\epsilon_{\sigma}(\ell)=\breve{\eta}\left(\mathbb{F}_{\ell}\right)$ and such that $\operatorname{ord}_{p}\left(\Psi_{w}\left(s_{2}\right)\right) \leq n^{\prime}$ is $1-1 / p^{n^{\prime}+1}$ for $0 \leq n^{\prime}<n$. Then by Notation 5.11 (2), there is a strongly $(n,-)$-admissible prime $\ell_{2}$ such that $\operatorname{ord}_{p}\left(\operatorname{loc}_{\ell_{2}}\left(s_{2}\right)\right) \leq n_{\text {den }}$ as we see in Lemma 5.7.

If we proceed as for the case under (6Ra), then we will obtain a contradiction when $n>n_{\text {div }}+n_{\text {red }}+n_{\text {den }}+n_{\text {bad }}$.

Lemma 5.16. If $\bar{\Delta}_{\sigma \otimes \breve{\eta}}^{n}$ is non-torsion, then $\operatorname{dim}_{\mathbb{Q}_{p}} \mathrm{H}^{1}\left(\mathbb{Q}, \mathrm{V}_{\sigma \times \breve{\pi}}\right)=0$.

Proof. The proof is very similar to Lemma 5.15. We only sketch the procedure and omit the justification since they are close. Let $n$ be a sufficiently large integer which will be determined later.

We prove by contradiction, and hence assume that $\operatorname{dim}_{\mathbb{Q}_{p}} \mathrm{H}^{1}\left(\mathbb{Q}, \mathrm{V}_{\sigma \times \breve{\pi}}\right) \geq 1$. By Lemma 5.9 , we may take a free $\mathbb{Z} / p^{n}$-submodule $S_{1}\left(\operatorname{resp} . S_{2}\right)$ of $\mathrm{H}_{f}^{1}\left(\mathbb{Q}, \overline{\mathrm{T}}_{\sigma \otimes \breve{\eta}}^{n}\right)$ (resp. $\mathrm{H}_{f}^{1}\left(\mathbb{Q}, \overline{\mathrm{T}}_{\sigma \times \breve{\pi}}^{n}\right)$ ) of rank 1 with basis $\left\{s_{1}\right\}$ (resp. $\left.\left\{s_{2}\right\}\right)$. Moreover, we may assume that $\bar{\Delta}_{\sigma \otimes \breve{\eta}}^{n}=p^{n_{\text {div }}} s_{1}$.

We start from $\overline{\mathrm{T}}_{\sigma \otimes \breve{\eta}}^{n}$ and choose a strongly $(n,-)$-admissible prime $\ell_{1}$ such that $\bar{\rho}_{\sigma \times \breve{\pi}}^{n}\left(\mathrm{Fr}_{\ell_{1}}\right)$ has order coprime to $p$, and $\operatorname{ord}_{p}\left(\operatorname{loc}_{\ell_{1}}\left(\bar{\Delta}_{\sigma \otimes \breve{\eta}}^{n}\right)\right) \leq n_{\text {den }}+n_{\text {div }}$. We also have $\operatorname{loc}_{\ell_{1}}\left(s_{2}\right)=0$. Now choose a strongly $(n,+)$-admissible prime $\ell_{2}$ such that $\operatorname{ord}_{p}\left(\operatorname{loc}_{\ell_{2}}\left(s_{2}\right)\right)=n_{\text {red }}$. If we proceed as for the case under (6Ra), then we will obtain a contradiction when $n>n_{\text {div }}+n_{\text {den }}+n_{\text {red }}+n_{\text {bad }}$.

Lemma 5.17. If $\bar{\Delta}_{\sigma \otimes \breve{\eta}}^{n}$ is non-torsion, then $\operatorname{dim}_{\mathbb{Q}_{p}} \mathrm{H}^{1}\left(\mathbb{Q}, \mathrm{V}_{\sigma \otimes \breve{\eta}}\right)=1$.

Proof. We prove by contradiction, and hence assume that $\operatorname{dim}_{\mathbb{Q}_{p}} \mathrm{H}^{1}\left(\mathbb{Q}, \mathrm{V}_{\sigma \otimes \breve{\eta}}\right) \geq 2$. Let $n$ be a sufficiently large integer which will be determined later. By Lemma 5.9, we may take a free $\mathbb{Z} / p^{n}$-submodule $S$ of $\mathrm{H}_{f}^{1}\left(\mathbb{Q}, \overline{\mathrm{T}}_{\sigma \otimes \breve{\eta}}^{n}\right)$ of rank 2. Moreover, we may assume that $S$ has a basis $\left\{s_{1}, s_{2}\right\}$ such that $\bar{\Delta}_{\sigma \otimes \breve{\eta}}^{n}=p^{n_{\text {div }}} s_{1}$. As in Lemma 5.16, we may choose a strongly $(n,-)$-admissible prime $\ell_{1}$ such that $\operatorname{ord}_{p}\left(\operatorname{loc}_{\ell_{1}}\left(s_{1}\right)\right) \leq n_{\text {den }}$. Taking a suitable linear combination of $s_{1}, s_{2}$, we obtain an element $s_{2}^{\prime} \in S$ with $\operatorname{ord}_{p}\left(s_{2}^{\prime}\right)=0$ and $\operatorname{loc}_{\ell_{1}}\left(s_{2}^{\prime}\right)=0$. Choose another strongly $(n,-)$-admissible prime $\ell_{2}$ such that $\operatorname{ord}_{p}\left(\operatorname{loc}_{\ell_{2}}\left(s_{2}^{\prime}\right)\right) \leq n_{\mathrm{den}}$. The same argument for the case under (6Ra) will produce a contradiction when $n>n_{\text {div }}+2 n_{\text {den }}+n_{\text {bad }}$.

5.3. Application to elliptic curves. Now we go back to deduce both Theorem 1.3 and Theorem 1.5 from Theorem 3.17. Let $\sigma$ (resp. $\pi$ ) be the cuspidal automorphic representation of $B_{N^{-}}^{\times}(\mathbb{A})\left(\operatorname{resp}\right.$. $\left.\operatorname{Res}_{F / \mathbb{Q}} \mathrm{GL}_{2}(\mathbb{A})\right)$ associated to $E$ (resp. $A$ ) as in $\$ 1.1$. Then 
both $\sigma$ and $\pi$ have the trivial central character, and are non-dihedral by Assumption $1.1(\mathrm{E} 1)$.

For the elliptic curve $A$, we have three different types.

Type AI: $A$ and $A^{\theta}$ are not geometrically isogenous;

Type AII: $A$ and $A^{\theta}$ are geometrically isogenous, but $A^{\theta}$ is not isogenous to a (possibly trivial) quadratic twist of $A$;

Type B: $A^{\theta}$ is isogenous to a (possibly trivial) quadratic twist of $A$.

Note that $\pi$ is Asai-decomposable (Definition 3.7) if and only if $A$ is of Type B. In this case, we have an extra number field $\breve{F}$.

Definition 5.18 (Group $\mathbf{P}$ ). We say that a prime $p$ is good (with respect to the pair $(E, A))$ if

(P1): $p \geq 11$ and $p \neq 13$;

(P2): $p$ is coprime to $N \cdot \mathrm{Nm}_{F / \mathbb{Q}} \mathfrak{M} \cdot \operatorname{disc}(F)$;

(P3): the $\Gamma_{\mathbb{Q}^{-}}$-representation $E[p]\left(\mathbb{Q}^{\text {ac }}\right)$ is surjective;

(P4): the $\Gamma_{\mathbb{Q}^{-}}$-representation $E[p]\left(\mathbb{Q}^{\text {ac }}\right)$ is ramified at all primes $\ell \mid N^{-}$with $p \mid$ $\ell^{2}-1$;

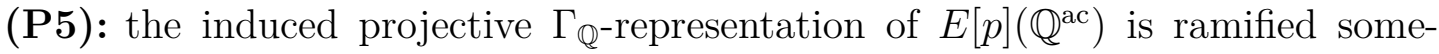
where outside $p$;

(P6): we have

- if $A$ is of Type AI, then the $\Gamma_{F}$-representation $A[p]\left(\mathbb{Q}^{\text {ac }}\right) \oplus A^{\theta}[p]\left(\mathbb{Q}^{\text {ac }}\right)$ has the largest possible image, that is, $\mathrm{G}\left(\mathrm{SL}\left(A[p]\left(\mathbb{Q}^{\mathrm{ac}}\right)\right) \times \mathrm{SL}\left(A^{\theta}[p]\left(\mathbb{Q}^{\mathrm{ac}}\right)\right)\right)$;

- if $A$ is of Type AII, then there exists an intermediate number field $F \subset K \subset$ $\mathbb{Q}^{\text {ac }}$ over which $A$ and $A^{\theta}$ are isogenous, such that the $\Gamma_{K}$-representation $A[p]\left(\mathbb{Q}^{\mathrm{ac}}\right)$ is surjective;

- if $A$ is of Type $\mathrm{B}$, then the $\Gamma_{\breve{F} F}$-representation $A[p]\left(\mathbb{Q}^{\text {ac }}\right)$ is surjective.

Lemma 5.19. For a given pair of elliptic curves $(E, A)$ satisfying Assumption 1.1, all but finitely many primes $p$ are good.

Proof. It is clear that for all but finitely many primes $p,(\mathrm{P} 1, \mathrm{P} 2, \mathrm{P} 4)$ are satisfied.

By [Ser72, Théorème 2], (P3) is also satisfied for all but finitely many primes $p$ under Assumption 1.1 (E1, E2).

(P6) is satisfied for all but finitely many primes $p$ by [Ser72, Théorème 6] (resp. [Ser72, Théorème 2]) if $A$ is of Type AI (resp. Type AI and Type B).

We prove (P5) by contradiction. Assume that (P5) does not hold for almost all primes $p$. Then the elliptic curve $E$ will have potentially good reduction at every prime $v \mid N$. For every prime $v \mid N$, let $\mathrm{J}_{v}$ be the smallest quotient of $\mathrm{I}_{v}$ such that the representation $\mathrm{V}_{q}(E)$ restricted to $\mathrm{I}_{v}$ factorizes through $\mathrm{J}_{v}$ for every prime $q \nmid N$. Then $\# \mathrm{~J}_{v}=2$. Denote by $\mathrm{I}_{v}^{\prime}$ the kernel of the projection $\mathrm{I}_{v} \rightarrow \mathrm{J}_{v}$. We may find a quadratic extension $F^{\prime} / \mathbb{Q}$ ramified at every prime $v \mid N$ with the prescribed inertia subgroup $\mathrm{I}_{v}^{\prime}$. It follows that $E$ acquires everywhere good reduction after base change to $F^{\prime}$, which violates [Kid02, Theorem 1.1].

Let $p$ be a good prime. We first check several conditions in Assumption 3.8. By enlarging the ideal $\mathfrak{M}$ to a positive integer $M$, but remaining coprime to $p N,(\mathrm{R} 1)$ is 
satisfied. (R2) is equivalent to (P3). (R3) is equivalent to (P4). (R5) is weaker than $(\mathrm{P} 1)$. The rest of this section will take care of (R4) and (R6).

Recall that we have the following Galois representations

$$
\rho_{\sigma}: \Gamma_{\mathbb{Q}} \rightarrow \mathrm{GL}\left(\mathrm{T}_{\sigma}\right), \quad \rho_{\pi}: \Gamma_{F} \rightarrow \mathrm{GL}\left(\mathrm{T}_{\pi}\right), \quad{ }^{\sharp} \rho_{\pi}: \Gamma_{\mathbb{Q}} \rightarrow \mathrm{GL}\left({ }^{\sharp} \mathrm{T}_{\pi}\right) .
$$

For each prime $\ell \nmid 2 p M \operatorname{disc}(F)$, the element ${ }^{\sharp} \rho_{\pi}\left(\mathrm{Fr}_{\ell}\right)$ has determinant 1 (resp. -1 ) if and only if $\ell$ is split (resp. inert) in $F$. Denote by $L_{1}$ (resp. $L_{2}$ ) the splitting field of the residue representation $\bar{\rho}_{\sigma}$ (resp. ${ }^{\sharp} \bar{\rho}_{\pi}$ ). Put $L_{0}=L_{1} \cap L_{2}$, in which the three fields are all number fields contained in $\mathbb{Q}^{\text {ac }}$. Denote by $\operatorname{GL}\left(\overline{\mathrm{T}}_{\sigma}\right)^{+}$the subgroup of $\mathrm{GL}\left(\overline{\mathrm{T}}_{\sigma}\right)$ of elements whose determinant belongs to $\left(\mathbb{F}_{p}^{\times}\right)^{2}$.

Lemma 5.20. The Galois group $\operatorname{Gal}\left(L_{1} / L_{0}\right)$ contains $\mathrm{GL}\left(\overline{\mathrm{T}}_{\sigma}\right)^{+}$.

Proof. We first show that $\operatorname{Gal}\left(L_{1} / L_{0}\right)$ contains $\operatorname{SL}\left(\overline{\mathrm{T}}_{\sigma}\right)$. Otherwise, the subgroup $\operatorname{Gal}\left(L_{1} / L_{0}\right)$ is contained in the center of $\operatorname{GL}\left(\overline{\mathrm{T}}_{\sigma}\right)$ since it is normal. Because ${ }^{\sharp} \rho_{\pi}$ is unramified outside $p M \operatorname{disc}(F)$, the fields $L_{2}$ and hence $L_{0}$ are unramified outside $p M \operatorname{disc}(F)$. In particular, for $v \mid N$, the element $\rho_{\sigma}\left(\mathrm{I}_{v}\right)$ is contained in the center of $\mathrm{GL}\left(\overline{\mathrm{T}}_{\sigma}\right)$. This is contradictory to $(\mathrm{P} 5)$.

By (P6), for every $a \in \mathbb{F}_{p}^{\times}$, the element $\left(a \mathbf{1}_{2}, a \mathbf{1}_{2}\right)$ is contained in the image of $\bar{\rho}_{\pi} \times \bar{\rho}_{\pi}^{\theta}$. Suppose that the previous element is the image of some Frobenius substitution Fr of $\mathbb{Q}^{\text {ac }}$, whose underlying prime is coprime to $p N M \operatorname{disc}(F)$ and split in $F$. Then ${ }^{\sharp} \bar{\rho}_{\pi}(\mathrm{Fr})=\mathbf{1}_{4}$ and $\operatorname{det} \bar{\rho}_{\sigma}(\mathrm{Fr})=a^{2}$. This means that $\operatorname{Gal}\left(L_{1} / L_{0}\right)$ contains an element of determinant $a^{2}$. Since $a$ is arbitrary, the lemma follows.

Remark 5.21. Lemma 5.20 implies the following

- If $A$ is of Type AI and $p$ is good, then $\mathrm{T}_{\sigma, \pi}$ is residually absolutely irreducible. - If $A$ is of Type $\mathrm{B}$ and $p$ is good, then $\mathrm{T}_{\sigma \times \breve{\pi}}$ is residually absolutely irreducible.

Now I claim that if $A$ is of Type AII, then $\mathrm{T}_{\sigma, \pi}$ is residually absolutely irreducible for all but finitely many (good) primes $p$. To indicate the relevance on $p$, we write $\mathrm{T}_{\pi, p}$ instead of $\mathrm{T}_{\pi}$. Assuming the contrary, for all but finitely many primes $p$, the representation $\overline{\mathrm{T}}_{\pi, p}$ is isomorphic to $\overline{\mathrm{T}}_{\pi, p}^{\theta} \otimes \breve{\eta}_{p}$ for a quadratic character $\breve{\eta}_{p}$, which a priori depends on $p$. Let $w$ be a place of $F$ whose underlying prime is split in $F$. Then for all but finitely many primes $p$,

$$
\operatorname{Sat}\left(\pi_{w}\right)^{2} \equiv \operatorname{Sat}\left(\pi_{w}^{\theta}\right)^{2} \bmod p,
$$

where Sat indicates the Satake parameter. Therefore, the character $\breve{\eta}:=\breve{\eta}_{p}$ is independent of $p$, and $\pi^{\theta} \simeq \pi \times \breve{\eta}$, which contradicts that $A$ is of Type AII. In particular, for all types of $A$, the integer $\mathfrak{n}(\mathrm{T})$ in Lemma 5.2 may be taken as 0 for $\mathrm{T}=\mathrm{T}_{\sigma, \pi}$ or $\mathrm{T}_{\sigma \times \breve{\pi}}$ for all but finitely many primes $p$.

$$
\text { Put } \mathbb{F}_{p}^{\text {bad }}=\left\{\mu \in \mathbb{F}_{p}^{\times} \mid \mu^{r}=1 \text { for some } r \in\{1,2,3,4,6\}\right\} \text {. }
$$

Lemma 5.22. The subgroup $\operatorname{Gal}\left(L_{2} / \mathbb{Q}\right)$ of $\mathrm{GL}\left({ }^{\sharp} \overline{\mathrm{T}}_{\pi}\right)$ contains a (semisimple) element whose geometric eigenvalues are $\left\{1,-1, \mu, \mu^{-1}\right\}$ for some $\mu \notin \mathbb{F}_{p}^{\text {bad }}$. 
Proof. For this statement, we may replace ${ }^{\sharp} \overline{\mathrm{T}}_{\pi}$ by the tensor induction (Asai representation) of $\overline{\mathrm{T}}_{\pi}$ since they have the same semisimplification. Choose a lifting $\vartheta \in \Gamma_{\mathbb{Q}}$ of $\theta \in \operatorname{Gal}(F / \mathbb{Q})$. We will use $\{1, \vartheta\}$ as the coset representatives to define the tensor induction, which is realized on the $\mathbb{F}_{p^{-}}$vector space $\overline{\mathrm{T}}_{\pi}^{\otimes 2}$. Suppose that

$$
\bar{\rho}_{\pi}\left(\vartheta^{2}\right)=\left(\begin{array}{ll}
a & b \\
c & d
\end{array}\right)
$$

under a basis $\left\{e_{1}, e_{2}\right\}$ of $\overline{\mathrm{T}}_{\pi}$. Then

$$
{ }^{\sharp} \bar{\rho}_{\pi}(\vartheta)=\varepsilon(\vartheta)^{-1}\left(\begin{array}{cccc}
a & b & & \\
& & a & b \\
c & d & & \\
& & c & d
\end{array}\right)
$$

under the basis $\left\{e_{1} \otimes e_{1}, e_{1} \otimes e_{2}, e_{2} \otimes e_{1}, e_{2} \otimes e_{2}\right\}$, where $\varepsilon(\vartheta)$ is an element in $\mathbb{F}_{p}^{\times}$whose square equals $\operatorname{det} \bar{\rho}_{\pi}\left(\vartheta^{2}\right)$. If $\varepsilon(\vartheta)^{-1} \bar{\rho}_{\pi}\left(\vartheta^{2}\right)$ has geometric eigenvalues $\left\{\mu, \mu^{-1}\right\}$ for some $\mu \notin \mathbb{F}_{p}^{\mathrm{bad}}$, then we are done. Otherwise, we may modify $\left\{e_{1}, e_{2}\right\}$ such that

$$
\varepsilon(\vartheta)^{-1} \bar{\rho}_{\pi}\left(\vartheta^{2}\right) \in\left\{\left(\begin{array}{ll}
\mu & \\
& \mu^{-1}
\end{array}\right), \pm\left(\begin{array}{ll}
1 & b \\
0 & 1
\end{array}\right) \mid \mu \in \mathbb{F}_{p}^{\text {bad }}, b \in \mathbb{F}_{p}\right\} .
$$

By (P1), we may choose some $\alpha \in \mathbb{F}_{p}^{\times}$whose order is not in $\{1,2,3,4,6,8,12,24\}$. Then $\mu \alpha^{2} \notin \mathbb{F}_{p}^{\text {bad }}$ for every $\mu \in \mathbb{F}_{p}^{\text {bad }}$. If we can show that $\operatorname{Gal}\left(L_{2} / \mathbb{Q}\right)$ contains the element

$$
\left(\begin{array}{cc}
\alpha & 0 \\
0 & \alpha^{-1}
\end{array}\right)^{\otimes 2}
$$

then we are done. The rest of the proof is dedicated to this point, according to types of $A$.

- If $A$ is of Type AI, then it follows from (P6).

- If $A$ is of Type AII, then we choose a number field $K$ as in (P6). Note that $\bar{\rho}_{\pi}^{\theta}(\gamma)=\bar{\rho}_{\pi}\left(\vartheta \gamma \vartheta^{-1}\right)$. Since $A_{K}$ is isogenous to $A_{K}^{\theta}$, the representations $\left.\bar{\rho}_{\pi}^{\theta}\right|_{\Gamma_{K}}$ and $\left.\bar{\rho}_{\pi}\right|_{\Gamma_{K}}$ are conjugate. By (P6), we may replace $\vartheta$ by its conjugation by some element in $\Gamma_{K}$, such that $\bar{\rho}_{\pi}\left(\vartheta \gamma \vartheta^{-1}\right)=\bar{\rho}_{\pi}(\gamma)$ for all $\gamma \in \Gamma_{K}$. Note that for $\gamma \in \Gamma_{K}$, we have ${ }^{\sharp} \bar{\rho}_{\pi}(\gamma)=\bar{\rho}_{\pi}(\gamma) \otimes \bar{\rho}_{\pi}\left(\vartheta \gamma \vartheta^{-1}\right)=\bar{\rho}_{\pi}(\gamma) \otimes \bar{\rho}_{\pi}(\gamma)$. The lemma follows.

- If $A$ is of Type B, then the proof is the same as above by letting $K=\breve{F} F$.

Lemma 5.23. Suppose that $p$ is a good prime as before. Then Assumption 3.8 (R6) is satisfied.

Proof. Suppose that $A$ is of Type AI or AII. By Lemma 5.20, the geometric irreducibility of ${ }^{\sharp} \mathrm{V}_{\pi}$ implies that of $\mathrm{V}_{\sigma, \pi}$. But $\left.{ }^{\sharp} \mathrm{V}_{\pi}\right|_{\Gamma_{F}} \simeq \mathrm{V}_{\pi} \otimes \mathrm{V}_{\pi}^{\theta}$ is absolutely reducible only when $\pi$ is Asai-decomposable, which is not the case.

Suppose that $A$ is of Type B. By Lemma 5.20, the geometric irreducibility of $\mathrm{V}_{\breve{\pi}}$ implies that of $\mathrm{V}_{\sigma \times \breve{\pi}}$. Note that $\left.\mathrm{V}_{\breve{\pi}}\right|_{\Gamma_{F}}$ is isomorphic to a quadratic twist of $\mathrm{Sym}^{2} \mathrm{~V}_{\pi}$, and the latter is absolutely irreducible since $\pi$ is non-dihedral. 
Again by Lemma 5.20, the image of $\bar{\rho}_{\sigma, \pi}$ contains a non-trivial scalar matrix. Then its Teichmüller lift will be the non-trivial scalar matrix of order dividing $p-1$ contained in the image of $\rho_{\sigma, \pi}$ or $\rho_{\sigma \times \breve{\pi}}$.

Lemma 5.24. Suppose that $p$ is a good prime as before. Then Assumption 3.8 (R4) is satisfied.

Proof. For this statement, we may again replace ${ }^{\sharp} \overline{\mathrm{T}}_{\pi}$ by the tensor induction of $\overline{\mathrm{T}}_{\pi}$. By Lemma 5.22, we fix an element $\gamma$ in the image of $\sharp \bar{\rho}_{\pi}$ whose geometric eigenvalues are $\left\{1,-1, \mu, \mu^{-1}\right\}$ for some $\mu \notin \mathbb{F}_{p}^{\text {bad }}$. By (P1), it is elementary to check that there is an element $b \in \mathbb{F}_{p}^{\times}$such that $b^{4} \notin\{1,-1, \mu-1,-\mu-1\}$.

Suppose that $A$ is of Type AI or AII. Then $\breve{F}=\mathbb{Q}$ in this case. By Lemma 5.20, the image of $\bar{\rho}_{\sigma} \times{ }^{\sharp} \bar{\rho}_{\pi}$ contains (some conjugate of)

$$
\left(\left(\begin{array}{ll}
\epsilon 1 & \\
& \epsilon b^{2}
\end{array}\right), \gamma\right)
$$

for both $\epsilon= \pm$. Then every prime $\ell \nmid 2 p q N^{-} \mathcal{D}_{N^{+} M}$ such that $\left(\bar{\rho}_{\sigma} \times{ }^{\sharp} \bar{\rho}_{\pi}\right)\left(\operatorname{Fr}_{\ell}\right)$ is conjugate to the above element will be a strongly $(1, \epsilon)$-admissible prime.

Suppose that $A$ is of Type B. Since $\breve{F}$ is unramified outside $M \operatorname{disc}(F)$, we have a similar result for $\overline{\mathrm{T}}_{\sigma \otimes \breve{\eta}}$ as in Lemma 5.20. Then the remaining argument is the same as the previous case.

Theorems 1.3 and 1.5 are finally proved!

\section{REFERENCES}

[SGA7I] Groupes de monodromie en géométrie algébrique. I, Lecture Notes in Mathematics, Vol. 288, Springer-Verlag, Berlin, 1972 (French). Séminaire de Géométrie Algébrique du BoisMarie 1967-1969 (SGA 7 I); Dirigé par A. Grothendieck. Avec la collaboration de M. Raynaud et D. S. Rim. MR0354656 (50 \#7134) $\uparrow 31$

[BG99] E. Bachmat and E. Z. Goren, On the non-ordinary locus in Hilbert-Blumenthal surfaces, Math. Ann. 313 (1999), no. 3, 475-506, DOI 10.1007/s002080050270. MR1678541 (2000b:14058) $\uparrow 25$

[BD05] M. Bertolini and H. Darmon, Iwasawa's main conjecture for elliptic curves over anticyclotomic $\mathbb{Z}_{p}$-extensions, Ann. of Math. (2) 162 (2005), no. 1, 1-64, DOI 10.4007/annals.2005.162.1. MR2178960 (2006g:11218) $112,30,39,40,41$

[BD07] M. Bertolini and H. Darmon, Hida families and rational points on elliptic curves, Invent. Math. 168 (2007), no. 2, 371-431, DOI 10.1007/s00222-007-0035-4. MR2289868 (2008c:11076) $\uparrow 36$

[BK90] S. Bloch and K. Kato, L-functions and Tamagawa numbers of motives, The Grothendieck Festschrift, Vol. I, Progr. Math., vol. 86, Birkhäuser Boston, Boston, MA, 1990, pp. 333400. MR1086888 (92g:11063) $\uparrow 30$

[BLR90] S. Bosch, W. Lütkebohmert, and M. Raynaud, Néron models, Ergebnisse der Mathematik und ihrer Grenzgebiete (3) [Results in Mathematics and Related Areas (3)], vol. 21, Springer-Verlag, Berlin, 1990. MR1045822 (91i:14034) $\uparrow 52$

[BC91] J.-F. Boutot and H. Carayol, Uniformisation p-adique des courbes de Shimura: les théorèmes de Čerednik et de Drinfel'd, Astérisque 196-197 (1991), 7, 45-158 (1992) (French, with English summary). Courbes modulaires et courbes de Shimura (Orsay, 1987/1988). MR1141456 (93c:11041) $\uparrow 20$ 
[BCDT01] C. Breuil, B. Conrad, F. Diamond, and R. Taylor, On the modularity of elliptic curves over Q: wild 3-adic exercises, J. Amer. Math. Soc. 14 (2001), no. 4, 843-939 (electronic), DOI 10.1090/S0894-0347-01-00370-8. MR1839918 (2002d:11058) $\uparrow 3$

[BM02] C. Breuil and W. Messing, Torsion étale and crystalline cohomologies, Astérisque 279 (2002), 81-124. Cohomologies $p$-adiques et applications arithmétiques, II. MR1922829 (2004k:14027) $\uparrow 46$

[BL84] J.-L. Brylinski and J.-P. Labesse, Cohomologie d'intersection et fonctions L de certaines variétés de Shimura, Ann. Sci. École Norm. Sup. (4) 17 (1984), no. 3, 361-412 (French). MR777375 (86i:11026) $\uparrow 33$

[BFH90] D. Bump, S. Friedberg, and J. Hoffstein, Nonvanishing theorems for L-functions of modular forms and their derivatives, Invent. Math. 102 (1990), no. 3, 543-618, DOI 10.1007/BF01233440. MR1074487 (92a:11058) $\uparrow 10$

[DR14] H. Darmon and V. Rotger, Diagonal cycles and Euler systems I: A p-adic Gross-Zagier formula, Ann. Sci. Éc. Norm. Supér. (4) 47 (2014), no. 4, 779-832 (English, with English and French summaries). MR3250064 $\uparrow 5$

[DRII] - Diagonal cycles and Euler systems II: the Birch and Swinnerton-Dyer conjecture for Hasse-Weil-Artin L-series, available at http://www.math.mcgill.ca/darmon/pub/pub.html. preprint. $\uparrow 5$

[Del80] P. Deligne, La conjecture de Weil. II, Inst. Hautes Études Sci. Publ. Math. 52 (1980), 137-252 (French). MR601520 (83c:14017) $\uparrow 31$

[FLHS] N. Freitas, B. V. Le Hung, and S. Siksek, Elliptic curves over real quadratic fields are modular, Invent. Math., available at http://link.springer.com/article/10.1007\%2Fs00222-014-0550-z. $\uparrow 3$

[Fuj02] K. Fujiwara, A proof of the absolute purity conjecture (after Gabber), Algebraic geometry 2000, Azumino (Hotaka), Adv. Stud. Pure Math., vol. 36, Math. Soc. Japan, Tokyo, 2002, pp. 153-183. MR1971516 (2004d:14015) $\uparrow 34$

[GGP12] W. T. Gan, B. H. Gross, and D. Prasad, Symplectic local root numbers, central critical L values, and restriction problems in the representation theory of classical groups, Astérisque 346 (2012), 1-109 (English, with English and French summaries). Sur les conjectures de Gross et Prasad. I. MR3202556 $\uparrow 7$

[Gar87] P. B. Garrett, Decomposition of Eisenstein series: Rankin triple products, Ann. of Math. (2) 125 (1987), no. 2, 209-235, DOI 10.2307/1971310. MR881269 (88m:11033) $\uparrow 3$

[vdG88] G. van der Geer, Hilbert modular surfaces, Ergebnisse der Mathematik und ihrer Grenzgebiete (3) [Results in Mathematics and Related Areas (3)], vol. 16, Springer-Verlag, Berlin, 1988. MR930101 (89c:11073) $\uparrow 24$

[Gor02] E. Z. Goren, Lectures on Hilbert modular varieties and modular forms, CRM Monograph Series, vol. 14, American Mathematical Society, Providence, RI, 2002. With the assistance of Marc-Hubert Nicole. MR1863355 (2003c:11038) $115,22,23$

[GO00] E. Z. Goren and F. Oort, Stratifications of Hilbert modular varieties, J. Algebraic Geom. 9 (2000), no. 1, 111-154. MR1713522 (2000g:14034) $\uparrow 25,28$

[Gro91] B. H. Gross, Kolyvagin's work on modular elliptic curves, L-functions and arithmetic (Durham, 1989), London Math. Soc. Lecture Note Ser., vol. 153, Cambridge Univ. Press, Cambridge, 1991, pp. 235-256, DOI 10.1017/CBO9780511526053.009, (to appear in print). MR1110395 (93c:11039) $\uparrow 57$

[GK93] B. H. Gross and K. Keating, On the intersection of modular correspondences, Invent. Math. 112 (1993), no. 2, 225-245, DOI 10.1007/BF01232433. MR1213101 (94h:11046) $\uparrow 8$

[GK92] B. H. Gross and S. S. Kudla, Heights and the central critical values of triple product Lfunctions, Compositio Math. 81 (1992), no. 2, 143-209. MR1145805 (93g:11047) ^4, 8

[GP12] B. H. Gross and J. A. Parson, On the local divisibility of Heegner points, Number theory, analysis and geometry, Springer, New York, 2012, pp. 215-241, DOI 10.1007/978-1-46141260-1, (to appear in print). MR2867919 $\uparrow 39$ 
[GP92] B. H. Gross and D. Prasad, On the decomposition of a representation of $\mathrm{SO}_{n}$ when restricted to $\mathrm{SO}_{n-1}$, Canad. J. Math. 44 (1992), no. 5, 974-1002, DOI 10.4153/CJM-1992060-8. MR1186476 (93j:22031) $\uparrow 7$

[GS95] B. H. Gross and C. Schoen, The modified diagonal cycle on the triple product of a pointed curve, Ann. Inst. Fourier (Grenoble) 45 (1995), no. 3, 649-679 (English, with English and French summaries). MR1340948 (96e:14008) $\uparrow 4$

[GZ86] B. H. Gross and D. B. Zagier, Heegner points and derivatives of L-series, Invent. Math. 84 (1986), no. 2, 225-320, DOI 10.1007/BF01388809. MR833192 (87j:11057) 个11

[HLR86] G. Harder, R. P. Langlands, and M. Rapoport, Algebraische Zyklen auf Hilbert-BlumenthalFlächen, J. Reine Angew. Math. 366 (1986), 53-120 (German). MR833013 (87k:11066) $\uparrow 9$

[Hel07] D. Helm, On maps between modular Jacobians and Jacobians of Shimura curves, Israel J. Math. 160 (2007), 61-117, DOI 10.1007/s11856-007-0056-0. MR2342491 (2009e:11119) $\uparrow 41$

[Ich08] A. Ichino, Trilinear forms and the central values of triple product L-functions, Duke Math. J. 145 (2008), no. 2, 281-307, DOI 10.1215/00127094-2008-052. MR2449948 (2009i:11066) $\uparrow 38,39$

[Jan88] U. Jannsen, Continuous étale cohomology, Math. Ann. 280 (1988), no. 2, 207-245, DOI 10.1007/BF01456052. MR929536 (89a:14022) 个33

[Jan90] _ Mixed motives and algebraic K-theory, Lecture Notes in Mathematics, vol. 1400, Springer-Verlag, Berlin, 1990. With appendices by S. Bloch and C. Schoen. MR1043451 (91g:14008) $\uparrow 6$

[dJ96] A. J. de Jong, Smoothness, semi-stability and alterations, Inst. Hautes Études Sci. Publ. Math. 83 (1996), 51-93. MR1423020 (98e:14011) 个31

[Kid02] M. Kida, Potential good reduction of elliptic curves, J. Symbolic Comput. 34 (2002), no. 3, 173-180, DOI 10.1006/jsco.2002.0555. MR1935076 (2003j:11060) $\uparrow 65$

[Kol90] V. A. Kolyvagin, Euler systems, The Grothendieck Festschrift, Vol. II, Progr. Math., vol. 87, Birkhäuser Boston, Boston, MA, 1990, pp. 435-483. MR1106906 (92g:11109) $\uparrow 2,11$

[LR98] E. Lapid and J. Rogawski, On twists of cuspidal representations of GL(2), Forum Math. 10 (1998), no. 2, 175-197, DOI 10.1515/form.10.2.175. MR1611951 (99d:11055) $\uparrow 32$

[LH14] B. Le Hung Viet, Modularity of some elliptic curves over totally real fields, ProQuest LLC, Ann Arbor, MI, 2014. Thesis (Ph.D.)-Harvard University. MR3251352 ^3

[Mil80] J. S. Milne, Étale cohomology, Princeton Mathematical Series, vol. 33, Princeton University Press, Princeton, N.J., 1980. MR559531 (81j:14002) ^34

[Mil06] _ Arithmetic duality theorems, 2nd ed., BookSurge, LLC, Charleston, SC, 2006. MR2261462 (2007e:14029) $\uparrow 31$

[Mur90] J. P. Murre, On the motive of an algebraic surface, J. Reine Angew. Math. 409 (1990), 190-204, DOI 10.1515/crll.1990.409.190. MR1061525 (91g:14003) 个7

[Nek00] J. Nekovář, p-adic Abel-Jacobi maps and p-adic heights, The arithmetic and geometry of algebraic cycles (Banff, AB, 1998), CRM Proc. Lecture Notes, vol. 24, Amer. Math. Soc., Providence, RI, 2000, pp. 367-379. MR1738867 (2002e:14011) ^36

[Nic00] M.-H. Nicole, The supersingular locus of Hilbert modular surfaces modulo p, 2000. Thesis (Ph.D.)-McGill University. $\uparrow 19,25$

[Niz93] W. Nizioł, Cohomology of crystalline representations, Duke Math. J. 71 (1993), no. 3, 747-791, DOI 10.1215/S0012-7094-93-07128-1. MR1240602 (94g:11045) 446

[PW11] R. Pollack and T. Weston, On anticyclotomic $\mu$-invariants of modular forms, Compos. Math. 147 (2011), no. 5, 1353-1381, DOI 10.1112/S0010437X11005318. MR2834724 (2012k:11174) $\uparrow 40$

[Pra92] D. Prasad, Invariant forms for representations of $\mathrm{GL}_{2}$ over a local field, Amer. J. Math. 114 (1992), no. 6, 1317-1363, DOI 10.2307/2374764. MR1198305 (93m:22011) 个3, 7, 8, 38 
[PSR87] I. Piatetski-Shapiro and S. Rallis, Rankin triple L functions, Compositio Math. 64 (1987), no. 1, 31-115. MR911357 (89k:11037) 个3, 39

[Rap78] M. Rapoport, Compactifications de l'espace de modules de Hilbert-Blumenthal, Compositio Math. 36 (1978), no. 3, 255-335 (French). MR515050 (80j:14009) 个22, 23

[RZ82] M. Rapoport and T. Zink, Über die lokale Zetafunktion von Shimuravarietäten. Monodromiefiltration und verschwindende Zyklen in ungleicher Charakteristik, Invent. Math. 68 (1982), no. 1, 21-101, DOI 10.1007/BF01394268 (German). MR666636 (84i:14016) $\uparrow 31$

[Rib89] K. A. Ribet, Bimodules and abelian surfaces, Algebraic number theory, Adv. Stud. Pure Math., vol. 17, Academic Press, Boston, MA, 1989, pp. 359-407. MR1097624 (92a:11070) $\uparrow 12,16,18,19,21,22,29$

[Rib90] K. A. Ribet, On modular representations of $\mathrm{Gal}(\overline{\mathbf{Q}} / \mathbf{Q})$ arising from modular forms, Invent. Math. 100 (1990), no. 2, 431-476, DOI 10.1007/BF01231195. MR1047143 (91g:11066) 339

[SS10] S. Saito and K. Sato, A finiteness theorem for zero-cycles over p-adic fields, Ann. of Math. (2) 172 (2010), no. 3, 1593-1639, DOI 10.4007/annals.2010.172.1593. With an appendix by Uwe Jannsen. MR2726095 (2011m:14010) $\uparrow 54$

[Sch94] A. J. Scholl, Classical motives, Motives (Seattle, WA, 1991), Proc. Sympos. Pure Math., vol. 55, Amer. Math. Soc., Providence, RI, 1994, pp. 163-187. MR1265529 (95b:11060) $\uparrow 6$

[Ser72] J.-P. Serre, Propriétés galoisiennes des points d'ordre fini des courbes elliptiques, Invent. Math. 15 (1972), no. 4, 259-331 (French). MR0387283 (52 \#8126) $\uparrow 65$

[Ski14] C. Skinner, A converse to a theorem of Gross, Zagier, and Kolyvagin (2014). arXiv:math/1405.7294. $\uparrow 9$

[Tat76] J. Tate, Relations between $K_{2}$ and Galois cohomology, Invent. Math. 36 (1976), 257-274. MR0429837 (55 \#2847) $\uparrow 31$

[TW95] R. Taylor and A. Wiles, Ring-theoretic properties of certain Hecke algebras, Ann. of Math. (2) 141 (1995), no. 3, 553-572, DOI 10.2307/2118560. MR1333036 (96d:11072) $\uparrow 3$

[TX13] Y. Tian and L. Xiao, On Goren-Oort stratification for quaternionic Shimura varieties (2013). arXiv:math/1308.0790. $\uparrow 26$

[TX14] - Tate cycles on some quaternionic Shimura varieties mod $p$ (2014). arXiv:math/1410.2321. $\uparrow 15,25,47$

[Vig80] M.-F. Vignéras, Arithmétique des algèbres de quaternions, Lecture Notes in Mathematics, vol. 800, Springer, Berlin, 1980 (French). MR580949 (82i:12016) $\uparrow 16$

[Wi195] A. Wiles, Modular elliptic curves and Fermat's last theorem, Ann. of Math. (2) 141 (1995), no. 3, 443-551, DOI 10.2307/2118559. MR1333035 (96d:11071) $\uparrow 3,41$

[YZZ] X. Yuan, S. Zhang, and W. Zhang, Triple product L-series and Hirzebruch-Zagier cycles, available at https://web.math.princeton.edu/ shouwu/publications/triple2012.pdf. preprint. $\uparrow 8$

[Zha12] W. Zhang, On arithmetic fundamental lemmas, Invent. Math. 188 (2012), no. 1, 197-252, DOI 10.1007/s00222-011-0348-1. MR2897697 $\uparrow 8$

[Zha14] _ Selmer groups and the indivisibility of Heegner points, Camb. J. Math. 2 (2014), no. $2,191-253 . \uparrow 9,36$

[Zin02] T. Zink, The display of a formal p-divisible group, Astérisque 278 (2002), 127-248. Cohomologies $p$-adiques et applications arithmétiques, I. MR1922825 (2004b:14083) $\uparrow 27$

Department of Mathematics, Northwestern University, Evanston, IL 60208

E-mail address: liuyf@math.northwestern.edu 